Louisiana State University

LSU Digital Commons

2015

\title{
A Spatial Dynamic Model of Population Changes in a Vulnerable Coastal Environment
}

Kenan Li

Louisiana State University and Agricultural and Mechanical College

Follow this and additional works at: https://digitalcommons.Isu.edu/gradschool_dissertations

Part of the Environmental Sciences Commons

\section{Recommended Citation}

Li, Kenan, "A Spatial Dynamic Model of Population Changes in a Vulnerable Coastal Environment" (2015). LSU Doctoral Dissertations. 817.

https://digitalcommons.Isu.edu/gradschool_dissertations/817

This Dissertation is brought to you for free and open access by the Graduate School at LSU Digital Commons. It has been accepted for inclusion in LSU Doctoral Dissertations by an authorized graduate school editor of LSU Digital Commons. For more information, please contactgradetd@lsu.edu. 


\title{
A SPATIAL DYNAMIC MODEL OF POPULATION CHANGES IN A VULNERABLE COASTAL ENVIRONMENT
}

\author{
A Dissertation \\ Submitted to the Graduate Faculty of the \\ Louisiana State University and \\ Agricultural and Mechanical College \\ in partial fulfillment of the \\ requirements for the degree of \\ Doctor of Philosophy \\ in
}

The Department of Environmental Sciences

\author{
by \\ Kenan Li \\ B.S., Nankai University, 2009 \\ M.S., Louisiana State University, 2011 \\ December 2015
}




\section{ACKNOWLEDGEMENTS}

I would like to express my deep gratitude to Dr. Nina Lam, my Ph.D advisor, for her patient guidance, enthusiastic encouragement and useful critiques of this dissertation work. I would also like to express my great appreciation to Dr. Kenneth Rose, for his valuable and constructive suggestions during the planning and development of this dissertation work. Special thanks should also be given to Dr. Michael Leitner and Dr. Margaret Reams, for their willingness to spend their time on this dissertation work, and their advice and assistance in keeping my progress on schedule.

I would also like to extend my thanks to my parents for their support and encouragement throughout my Ph.D study. Last but not the least, I would like to thank all my fellow lab mates in the RSGIS lab, the ones who have been sharing insightful comments and exchanging various perspectives with me throughout my Ph.D study.

This research was funded in part by grants from the U.S. National Science Foundation under the Dynamics of Coupled Natural Human Systems (CNH) Program and the Coastal SEES Program (award numbers 1212112 and 1427389). The statements, findings, and conclusions are those of the authors and do not necessarily reflect the views of the funding agencies. 


\section{TABLE OF CONTENTS}

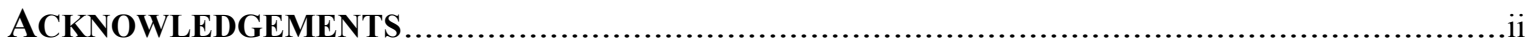

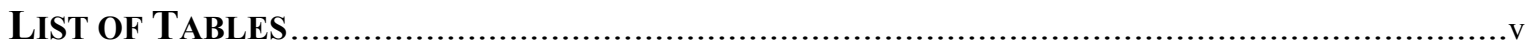

LIST OF FIGURES ...............................................................................................

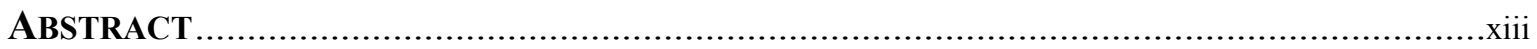

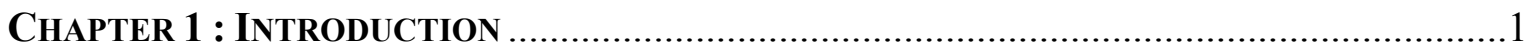

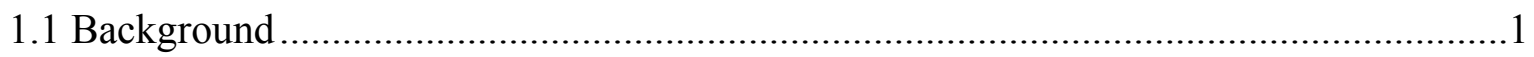

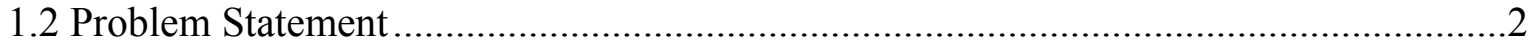

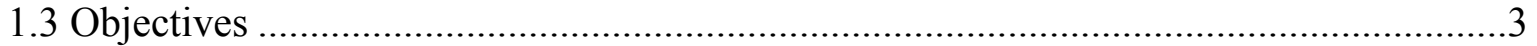

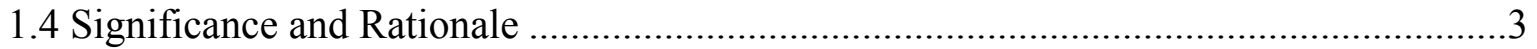

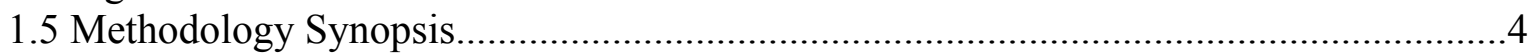

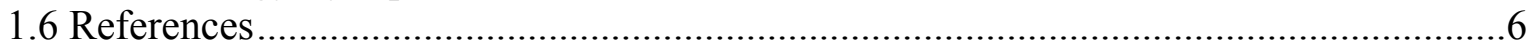

CHAPTER 2 : LITERATURE REVIEW AND CONCEPT FRAMEWORK DEVELOPMENT .............8

2.1 Coupled Natural and Human Dynamics and Complex Systems .....................................8

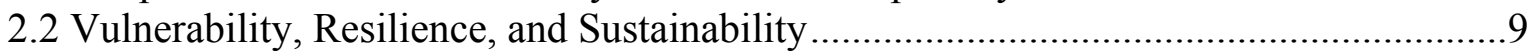

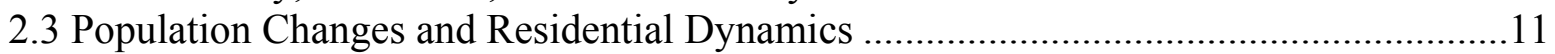

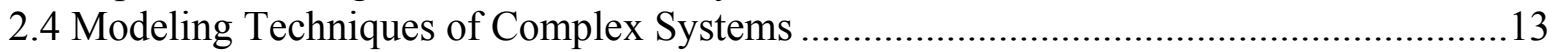

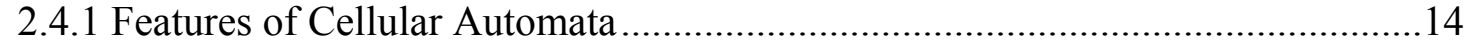

2.4.2 Features of Agent Based Modeling …………............................................15

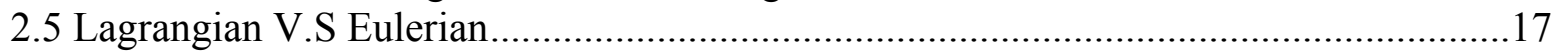

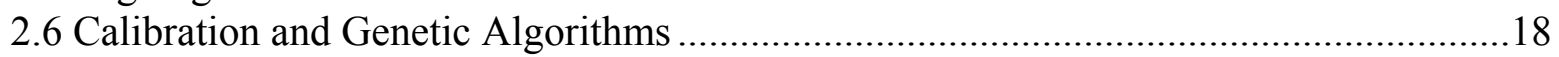

2.7 The Framework of the Spatial Dynamic Model …….................................................19

2.8 Development Platforms ………………………...................................................22

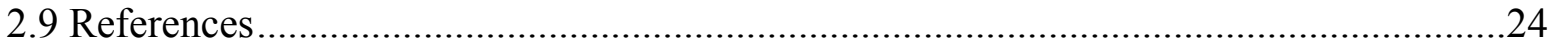

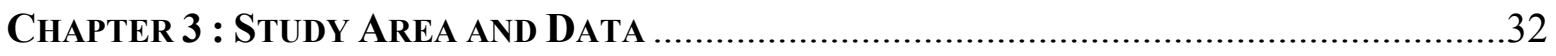

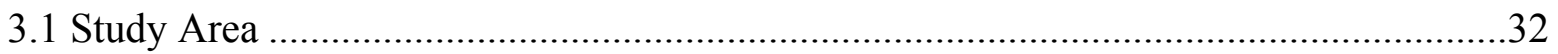

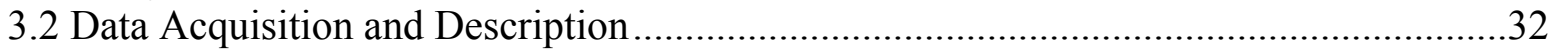

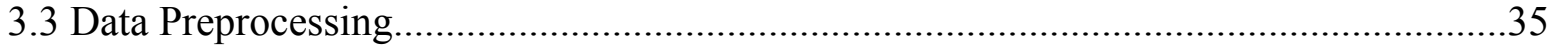

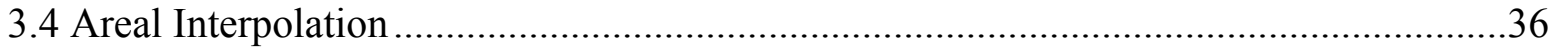

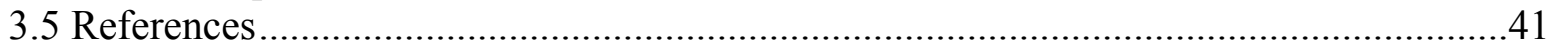

Chapter 4 : VARIABLE SELECTION AND RULES EXTRACTION ...........................................42

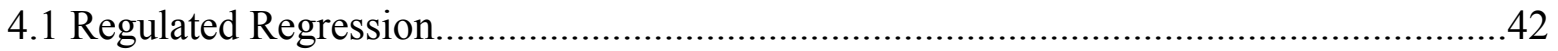

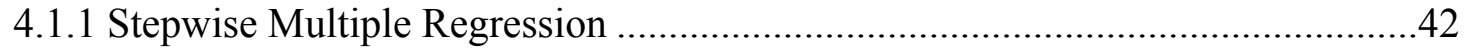

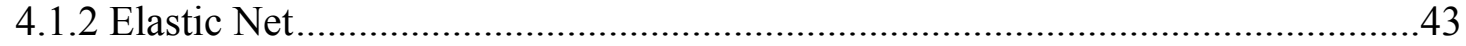

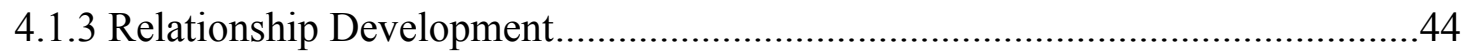

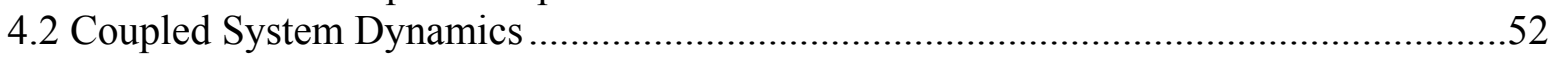

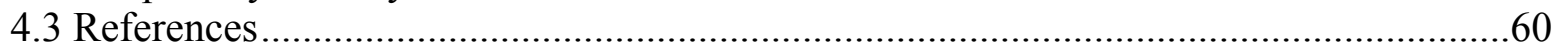


Chapter 5 : Spatial Danamic Model and Genetic Algorithim ...........................61

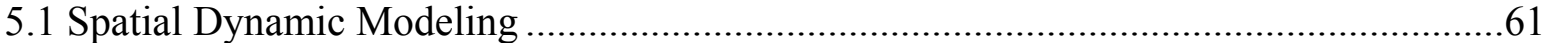

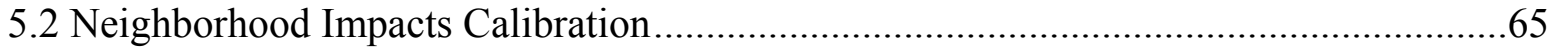

5.3 Monte Carlo Uncertainty Analysis ......................................................................... 73

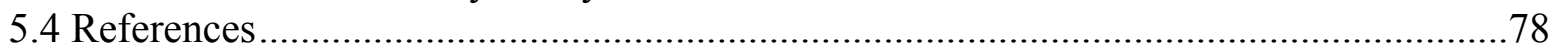

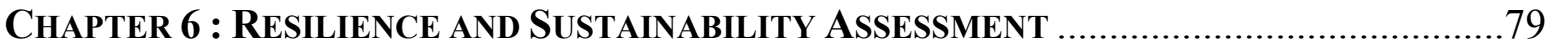

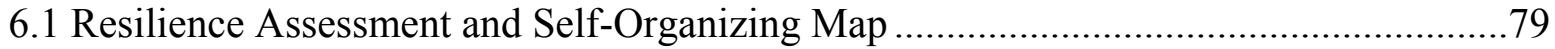

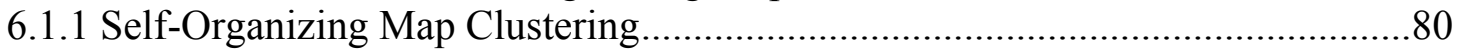

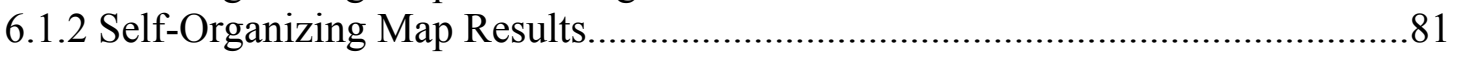

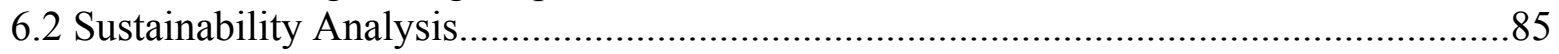

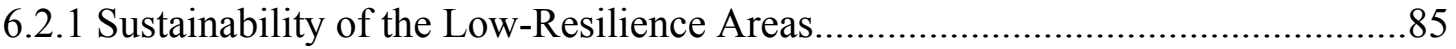

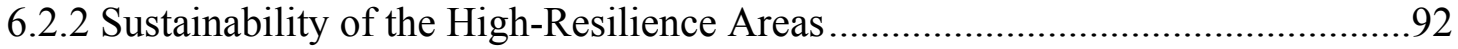

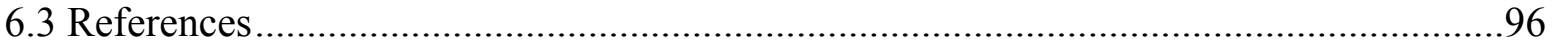

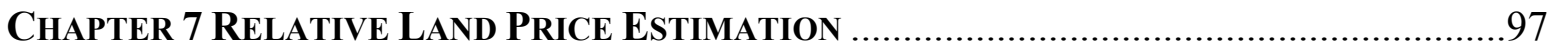

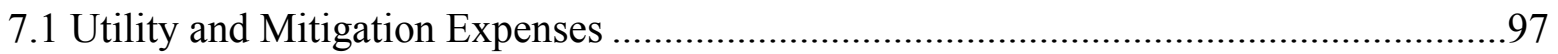

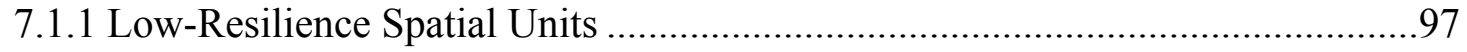

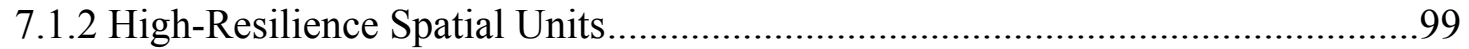

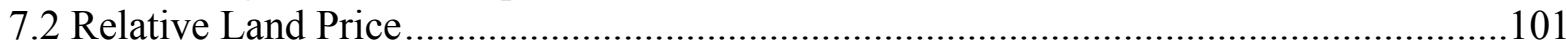

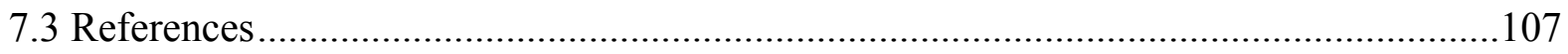

Chapter 8 COUPled Natural AND Human DyNAMics OF THE "NORTh" AND The

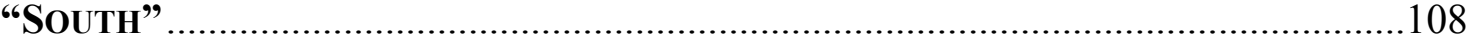

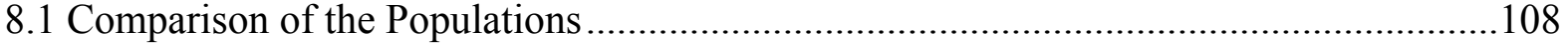

8.2 Comparison of the Growth of the Developed Areas..................................................111

8.3 Comparison of the Relative Land Prices .................................................................115

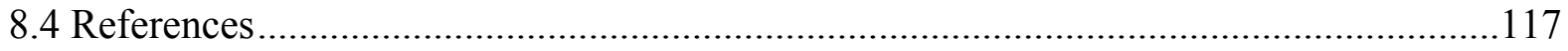

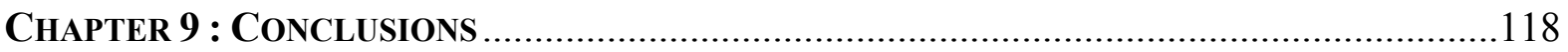

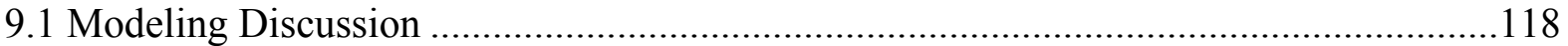

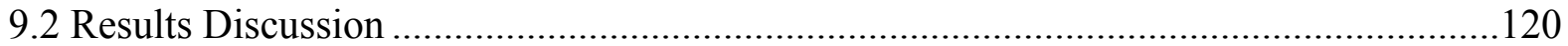

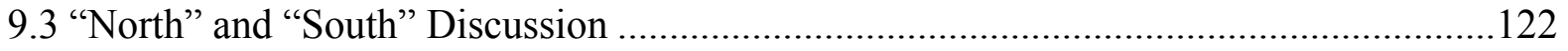

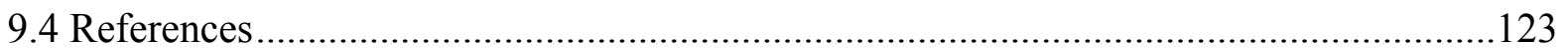

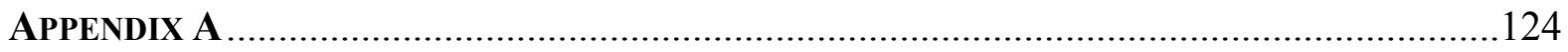

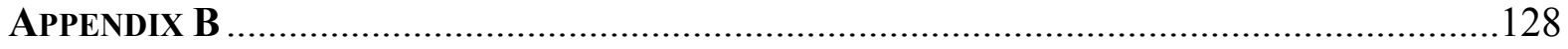

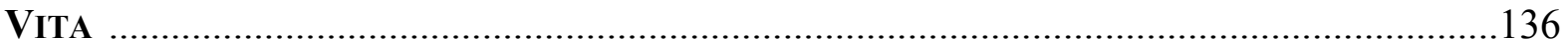




\section{LIST OF TABLES}

Table 3.1 Major Types of Coastal Hazards in this Study with Descriptions. 33

Table 3.2 Acronyms and Descriptions of the Socioeconomic and Housing Variables used in this Study. 34

Table 3.3 Acronyms and Descriptions of the Demographic and Environmental Variables used in this Study. 35

Table 4.1 Standardized Coefficients of the Variables Related to Population Change Selected By Elastic Net 46

Table 4.2 Standardized Coefficients of the Variables Related to Developed Land Area Change Selected By Elastic Net. 50

Table 4.3 Standardized Coefficients of the Variables Related to Utility Change Selected By Elastic Net 52

Table 4.4 Symbols, Units and Definitions for the Coefficients of the Equations in the Spatial Dynamic Model 54

Table 4.5 Acronyms, Types, Units, Definitions, and Updating Rules of the Variables in the Spatial Dynamic Model 55

Table 5.1 Confusion Matrix for Population Prediction of 2010 73

Table 5.2 Confusion Matrix for Developed Area Percentage Prediction of 2010. 73

Table 5.3 Probability Distribution Specification for the Monte Carlo Uncertainty Analysis (Unstandardized Real Value). 74

Table 5.4 Pearson Correlations between the Assessment Variables and the Selected Parameters 77

Table 6.1 Multi-Linear Regression Results for the Average Population of the Low-Resilience Spatial Units (on $\theta$ and Mitigation Expenses) 91 
Table 6.2 Multi-Linear Regression Results for the Average Population of the High Resilience

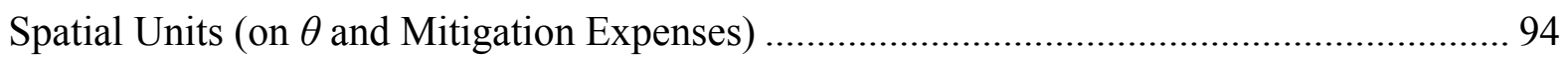

Table 7.1 Multi-Linear Regression Results for the Average Population of the Low Resilience

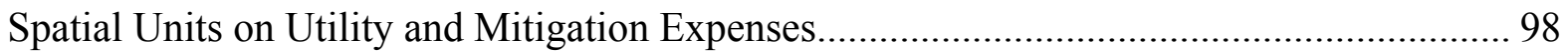
Table 7.2 Multi-Linear Regression Results for the Average Population of the High-Resilience Spatial Units on Utility and Mitigation Expenses............................................................. 100 Table 7.3 Multi-Linear Regression Results for the Average Population of all the Spatial Units in City Areas of the Study Area on Utility and Mitigation Expenses.................................. 105 


\section{LIST OF FIGURES}

Figure 1.1 The Study Area with the Hypothetical North-South Boundary, and its Population

Distribution in 2006 (LandScan Data) ............................................................................ 1

Figure 2.1 Structure of a Cellular Automata Model ...................................................... 15

Figure 2.2 Structure of an Agent Based Model ........................................................ 16

Figure 2.3 Lagrangian Framework V.S Eulerian Framework ....................................... 18

Figure 2.4 Structure of the Modeling Units of the Spatial Dynamic Model in this Study ..... 21

Figure 3.1 Spectrum of Semivarigrams of the Yearly Subsidence Rate in the Study Area ... 36

Figure 3.2 Areal Interpolation with Ancillary Layer in this study ................................. 38

Figure 3.3 Population Density of the Census Blocks in 2000 ......................................... 39

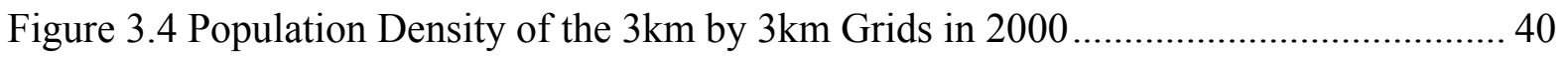

Figure 4.1 Cross Validated MSE of Elastic Net for Population Change ............................. 45

Figure 4.2 Trace Plot of Coefficients by Elastic Net for Population Change...................... 46

Figure 4.3 Standardized Coefficients of the Variables Related to Population Change Selected

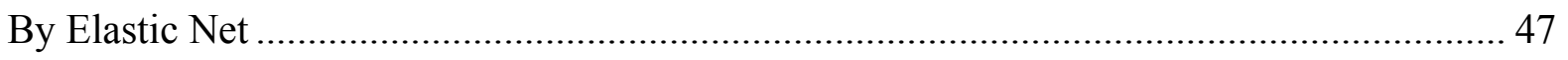

Figure 4.4 Cross Validated MSE of Elastic Net for Developed Land Area Percentage Change

Figure 4.5 Trace Plot of Coefficients by Elastic Net for Developed Land Area Percentage

Change .

Figure 4.6 Standardized Coefficients of the Variables Related to Developed Land Area Change

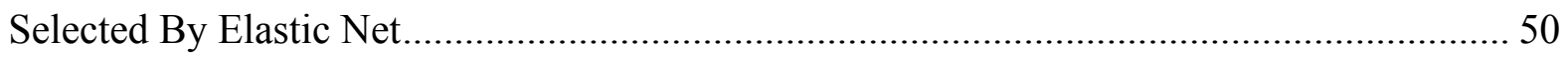

Figure 4.7 Cross Validated MSE of Elastic Net for Utility Change .................................. 51

Figure 4.8 Trace Plot of Coefficients by Elastic Net for Utility Change............................ 51 
Figure 4.9 Standardized Coefficients of the Variables Related to Utility Change Selected By Elastic Net 52

Figure 4.10 The Coupled System Dynamics Diagram ................................................. 53

Figure 4.11 Comparison between the Predicted and the Real Population (Standardized) in 2010 by Spatial Units (System Dynamics) 57

Figure 4.12 Comparison between the Predicted and the Real Developed Land Area Percentage (Standardized) in 2010 by Spatial Units (System Dynamics) 57

Figure 4.13 Comparison between the Predicted and the Real Population (Standardized) in 2010 by Spatial Units (Elastic Net) 58

Figure 4.14 Comparison between the Predicted and the Real Developed Land Area Percentage (Standardized) in 2010 by Spatial Units (Elastic Net). 58

Figure 5.1 The Spatial Coupled Dynamics Diagram 63

Figure 5.2 Comparison between the Predicted and the Real Population (Standardized) in 2010 by Spatial Units (with Neighborhood Effects). 64

Figure 5.3 Comparison between the Predicted and the Real Developed Land Area Percentage (Standardized) in 2010 by Spatial Units (with Neighborhood Effects) 64

Figure 5.4 Genetic Algorithm Results for Calibrating the Neighborhood Impacts..... 66

Figure 5.5 Total Population and Total Developed Area Percentage of all the Spatial Units (Standardized Values) in the Sensitivity Analysis of $\mathrm{P}_{1}, \mathrm{P}_{2}$, and $\mathrm{P}_{3}$ 68

Figure 5.6 Comparison between the Predicted and the Real Population (Standardized) in 2010 by Spatial Units (after GA Calibration) 69

Figure 5.7 Comparison between the Predicted and the Real Developed Land Area Percentage (Standardized) in 2010 by Spatial Units (after GA Calibration) 69 
Figure 5.8 Real Population of the Study Area in 2010 70

Figure 5.9 Population Projected by the Spatial Dynamic Model after Genetic Algorithms

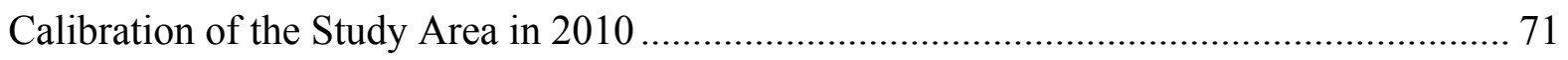

Figure 5.10 Real Developed Land Use Area Percentage of the Study Area in 2010 ........... 71

Figure 5.11 Developed Land Use Area Percentage Projected by the Spatial Dynamic Model after Genetic Algorithms Calibration of the Study Area in 2010 .................................... 72

Figure 5.12 Bin-Centers for the Histograms of Total Population...................................... 75

Figure 5.13 Bin-Centers for the Histograms of Total Developed Area Percentage .............. 75

Figure 5.14 Bin-Centers for the Histograms of Total Utility......................................... 76

Figure 6.1 Neighbor Weight Distances of the Neurons in the Self-Organizing Map............ 82

Figure 6.2 Number of the Spatial Units belonged to Each Neuron .................................... 82

Figure 6.3 Weight Positions of the Spatial Units and the Four Neurons in the Output Space of

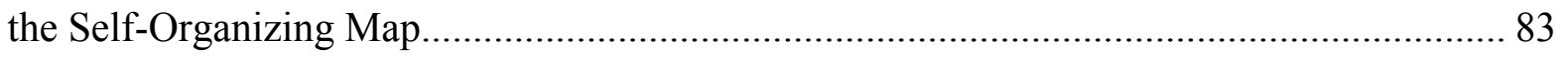

Figure 6.4 The Central Time Series for the Four Neurons (Clusters) ................................ 84

Figure 6.5 The Resilience Groups for all the Spatial Units in the City Areas of the Study Area 85

Figure 6.6 The Property Damages by Coastal Natural Hazards (Dollars) of the Spatial Units in

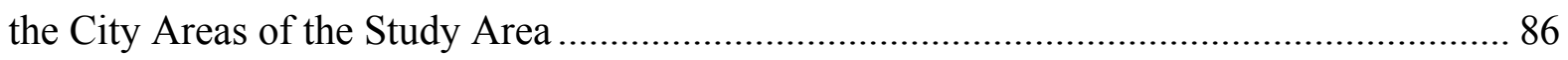

Figure 6.7 The Utility of the Spatial Units in the City Areas of the Study Area................. 87

Figure 6.8 Average Population of the Low-Resilience Spatial Units Simulated with Different

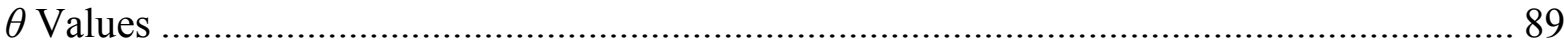

Figure 6.9 Average Population of the Low-Resilience Spatial Units Simulated with Different

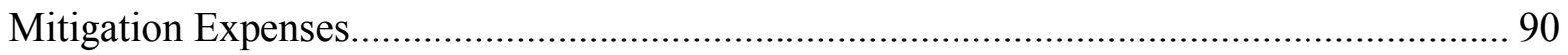


Figure 6.10 Sustainability Analysis of the Low-Resilience Spatial Units with Mitigation Budget Set to 150 Million Dollars per 10 Year and Sustainability Goal Set to 8000 People 92 Figure 6.11 Average Population of the High-Resilience Spatial Units Simulated with Different $\theta$ Values 93

Figure 6.12 Average Population of the High-Resilience Spatial Units Simulated with Different Mitigation Expenses. 93

Figure 6.13 Sustainability Analysis of the High Resilience Spatial Units with Mitigation Budget set to 150 Million Dollars and Sustainability Goal set to 1500 People. 95

Figure 7.1 Average Population of the Low-Resilience Spatial Units Simulated with Different Utility Increases 98

Figure 7.2 Average Population of the Low-Resilience Spatial Units for Different Utility and Mitigation Expenses. 99

Figure 7.3 Average Population of the High-Resilience Spatial Units Simulated with Different Utility Increases 100

Figure 7.4 Average Population of the High-Resilience Spatial Units for Different Utility and Mitigation Expenses. 101

Figure 7.5 The Relative Land Price of the High Resilience Spatial Units and the Relative Land Price of the Low Resilience Spatial Units 102

Figure 7.6 Average Population of all the Spatial Units in the City Areas of the Study Area Simulated with Different Mitigation Expenses. 104

Figure 7.7 Average Population of all the Spatial Units in the City Areas of the Study Area Simulated with Different Utility Increases 105 
Figure 7.8 The Relative Land Price of all the Spatial Units in the City Areas of the Study Area 106

Figure 8.1 The City Areas in the Study Area with the "North" and "South" Boundary ...... 108 Figure 8.2 Average Simulated Population of the Low-Resilience Areas in New Orleans and Baton Rouge and the Whole City Areas of New Orleans and Baton Rogue from 2010 to 2050 109

Figure 8.3 Average Simulated Population of the "North" City Areas and the "South" City Areas from 2010 to 2050 109

Figure 8.4 Average Simulated Population of the Low-Resilience Areas in New Orleans and Baton Rouge and the Whole City Areas of New Orleans and Baton Rogue from 2010 to 2210

Figure 8.5 Average Simulated Population of the "North" City Areas and the "South" City Areas from 2010 to 2210 110

Figure 8.6 Average Simulated Developed Land Use Area Percentage of the "North" Areas and the "South" Areas from 2010 to 2050. 112

Figure 8.7 Average Simulated Developed Land Use Area Percentage of the "North" Areas and the "South" Areas from 2010 to 2210. 112

Figure 8.8 Areas with their Simulated Developed Land Percentages increased by at Least 10\% 114

Figure 8.9 Percentages of Land Use Types of the North and the South Areas in 2010 with their Developed Land Percentages increased by at Least $10 \%$ by 2050 114

Figure 8.10 Average Simulated Relative Land Price of the "North" Areas and the "South" Areas from 2010 to 2050 115 
Figure 8.11 Average Simulated Relative Land Price of the "North" Areas and the "South" Areas from 2010 to 2210 


\begin{abstract}
Achieving coastal sustainability in low-lying coastal areas is a great challenge. This study developed a spatial dynamic model to study the coupled natural-human responses in the form of population changes in the Lower Mississippi River Basin region. The goal was to identify the key social-economic factors (utility) and selected environmental factors (such as hazards damage, elevation, and subsidence rate) that affect population changes, as well as how population changes affect the local utility and the local environment reciprocally. The study area was partitioned into the "north' and the "south" by a hypothetical boundary to test the differences of the emergence. Areal interpolation techniques with volume preserving property were used to integrate all the data acquired from different sources and defined in various formats into a unified $3 \mathrm{~km}$ by $3 \mathrm{~km}$ cellular space. An Elastic Net model was built to extract the rules and calibrate the parameters. Genetic Algorithms were applied to calibrate the neighborhood effects. A Monte Carlo approach using random sampling was used to conduct the uncertainty analysis. The final model yielded an accuracy of above $97 \%$ in projecting both the population changes and the developed area percentage changes from 2000 to 2010 .
\end{abstract}

A resilience assessment framework and a sustainability assessment framework were used to examine the simulated results from 2010 to 2050 . The low-resilience areas were found to concentrate in the "south" in the central metropolitan areas of New Orleans. The sustainability analysis shows that high-resilience areas will always be sustainable. However, for the low-resilience areas, three sustainability conditions can occur depending on the mitigation budget: the tipping space, the mitigatable space, and the sustainable space. A Relative Land Price concept was defined to indicate the surplus value of a spatial unit due to its population and utility. The low-resilience areas were found to have higher Relative Land Prices mainly due to their high populations. In the short time-period simulation (2010-2050), 
the "south" will fall behind the "north" in population growth and developed land increase, and its average population was projected to be decreasing. However, in the long time-period simulation (2010-2210), its average population is able to bounce back from a certain population level. The results from this study will shed light on the relationships between coastal hazards and human responses and provide valuable insight into the development of optimal strategies for coastal sustainability. 


\section{CHAPTER 1 : INTRODUCTION}

\subsection{Background}

Coastal areas have their unique benefits over inland areas, such as convenient access to coastal resources, marine transportations, oceanic crude oil explorations, and favorable climate for human living. At the same time, these areas are much more exposed to natural coastal hazards, including land loss, land subsidence, coastal erosion, coastal flooding, tsunami, sea level rise, and hurricanes. All these hazards have negative effects on the economic growth and social construction of the communities along the coast. In the United States, the Lower Mississippi River Basin (LMRB) in southern Louisiana is highly vulnerable to coastal hazards (Fig. 1). In the past ten years (2005-2015), the region has experienced at least five hurricanes (Katrina, Rita, Gustav, Ike, and Isaac), which caused significant loss of human lives and damages to properties (Lam et al., 2009a\&b, 2012a; LeSage et al., 2011 a, b\&c). With the impending threats of climate change and sea level rise, coastal Louisiana is facing a serious challenge, which is how to protect the land while maintaining economic growth, or in other words, how to achieve coastal sustainability.

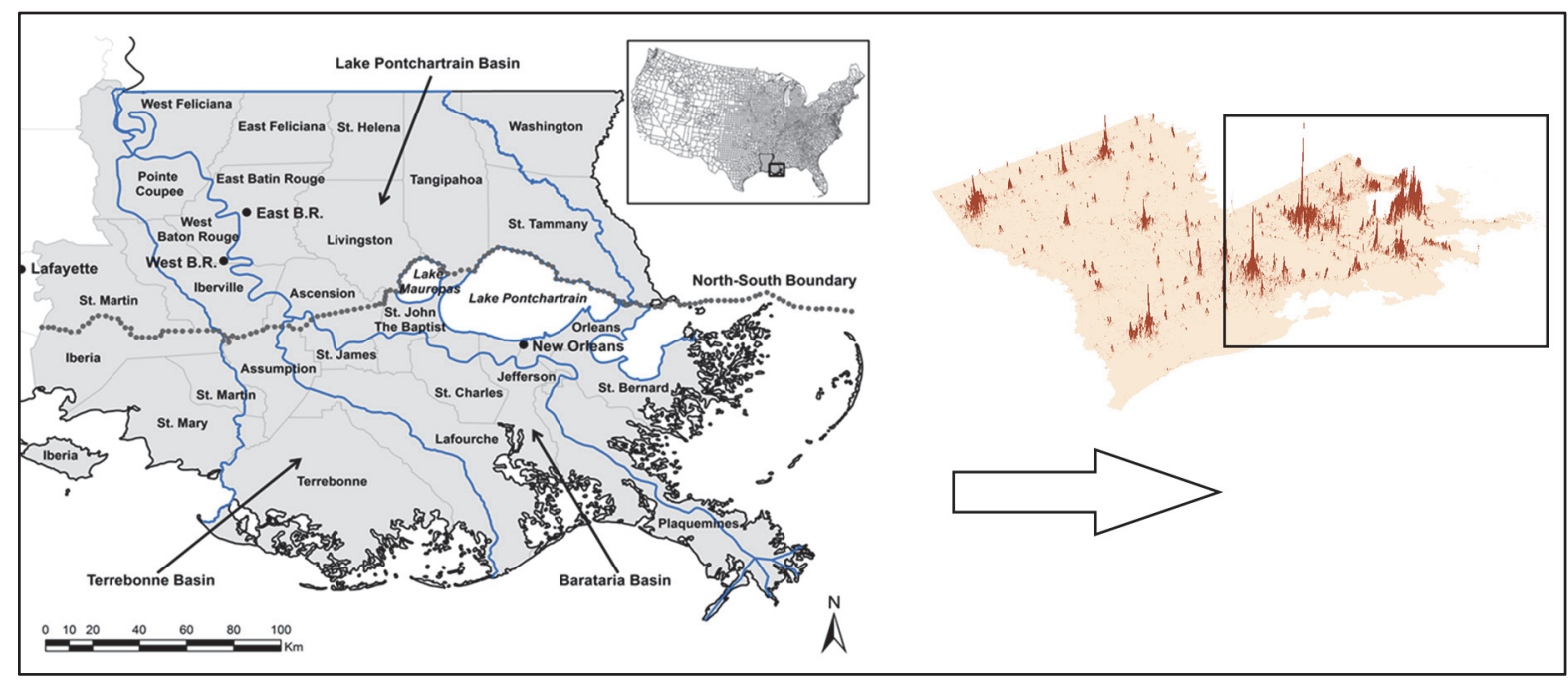

Figure 1.1 The Study Area with the Hypothetical North-South Boundary, and its Population Distribution in 2006 (LandScan Data) 


\subsection{Problem Statement}

There has been extensive research on the causes and consequences of increased vulnerability in the Lower Mississippi River Delta region (Blum \& Roberts, 2009). Significant efforts from governmental and non-governmental agencies have also been made to develop long-term master plans for coastal protection and restoration (Coastal Protection and Restoration Agency of Louisiana [CPRA], 2012). However, the existing literature on coastal protection and restoration has largely focused on understanding the natural environment, with fewer studies devoted to the human side. There is very few published literature on how the natural and human systems are coupled in this coastal environment. Studying how the natural and human environments interact is critical to a better understanding of the dynamics of the coastal vulnerability and sustainability problem (Liu J. et al., 2007a \&b; Collins et al. 2010; Kates, 2011).

Moreover, in the past decade, a gradual population growth has been observed in the northern of the Lower Mississippi River Delta, in contrast with a dramatic decline in its southern part (Lam et al., 2012b). Some of the increase in population has been documented as the migration from the southern part of the region (Plyer, 2013). This phenomenon is noteworthy in that it signals a voluntary migration that is not part of the governmental coastal restoration plan. In other words, people choose to move to places that are less subject to natural hazards while providing economic opportunities. They might also want to move to places that are not far from their original places to maintain their cultures and social network. With consideration of this autonomous human factor, the traditional "top-down" approach of coastal restoration may not be effective in achieving a long-term protection and the coastal sustainability, as people may find those places not "desirable" to live even if there are land protection and restoration. Therefore, in planning for coastal sustainability, we must consider these "bottom-up" facts so that we can formulate optimal strategies on where and how to protect the land and its resources while maintaining economic growth. 


\subsection{Objectives}

The overall objective of this dissertation research is to develop a spatial dynamic model to understand the coupled natural-human responses in the form of population changes in the Lower Mississippi River Basin. This study seeks to answer these related questions: (1) What are the key socio economic factors (utility) that trigger the population changes; (2) How important are the environmental factors (such as natural coastal hazards, elevation, subsidence rate, and so on) in affecting the population changes in the LMRB; (3) Reciprocally, how do the changes of population affect the local utility and the local natural environment, for example, by increasing the local developed land use areas; (4) How to evaluate the local resilience of this coupled natural and human dynamic system; (5) What will the future of the region likely be under different animated scenarios. The findings from these studies can provide valuable insight into the development of optimal strategies for coastal sustainability.

\subsection{Significance and Rationale}

This research is significant in four aspects. First, this study is one of the few studies that focus explicitly on simulating human population changes in the context of coastal resilience and coupled natural and human dynamics. The simulation helps increase our understanding of the complex coupling process, especially on what determines the population changes and how the changes reciprocally affect the local land units. The results from this study will shed light on the relationships between climate change, human responses, and environmental policies. Second, few studies have attempted to measure the resilience of human dynamics. This study developed a system dynamic model with focuses on quantifying local vulnerability and resilience, capturing the complex interactions between human and nature components, and empirically validating the proposed measures. Finally, the use of empirical data and evolutionary algorithms for calibration and validation is useful. A validated simulation model supported by the real data will allow policy makers to design and test sustainable policies so that resources can be better allocated to help build the local resilience. 
Although the simulation model was derived by the data of the Lower Mississippi River Basin focusing on the coast-hazard vulnerability, it can also be applied to other locations affected by natural disasters of the similar or other nature. Findings from this study can be used to support the decision-making in a broader range of sustainability planning, not only the coastal. A better understanding of how the human system responds to disaster events will allow policy makers to improve disaster preparedness plans. The simulation model will be a valuable tool for climate change planning. The model can also be used to predict future human residential location patterns under different hazard scenarios. The model results will help increase our understanding of what socioeconomic indicators would affect the vulnerability of a land unit the most, and what planning or aid strategies would be the best to help the land unit in preventing population from decreasing.

\subsection{Methodology Synopsis}

To address the above objectives, there are two major challenges: (1) what do we mean by "vulnerability" or "resilience" and how do we measure the sustainability, and (2) how can we define and model the internal reciprocal mechanism of the feedback loop between the natural and the human environments?

For the first challenge, the evaluation of "vulnerability" or "resilience" in this study was an extension of the study from the Resilience Inference Measurement (RIM) model (Lam et al., 2015 a\&b; Li K., 2011; Li K. et al. 2015; Li C., 2013). The final "resilience" capacity and the sustainability were assessed by the simulated population changes under different scenarios with different degrees of hypothesized disturbance from natural hazards.

For the second challenge, a set of coupled system dynamics were developed with the coupled feedback loops extracted from statistical models, and a spatially explicit dynamic model was developed to model the population changes with neighborhood effects. The spatial units in this model are $3 \mathrm{~km}$ by $3 \mathrm{~km}$ land cells. For each land cell, the aggregated population, the percentage of developed land use areas, and the other social, ecological, and environmental 
variables are homogeneous and considered as its characteristics. A set of coupled system dynamics was designed with these variables. Statistical methods such as Elastic Net (a regulated regression method, see Chapter 4) were used to extract the key variables and their parameters in the coupled dynamics. The coupled dynamics was simulated for each single spatial unit, with neighborhood effects added. The neighborhood effects was calibrated using Genetic Algorithms to match the real historic data.

Thus, this research consists of three stages. The first stage is to understand the process of population changes through statistical analyses so that rules and parameters used in the simulation model are as realistic as possible. These extracted rules and parameters should be able to represent the human and nature interaction process in this study area. The second stage is to use the system dynamics to create a "bottom-up" model so that autonomous emergence can be studied. The third is to further calibrate the parameters of the spatial dynamic model by using the evolutionary algorithm approach. Once the simulation model is calibrated and validated, the effects of local vulnerability and externality on the population changes, and how such effects propagate through time, can be estimated and visualized by the simulation model.

The following chapters are organized in the order according to the way that can best interpret the three stages. Chapter 2 reviews the recent literatures on the topics of resilience, coupled natural and human systems, and "bottom-up" modeling techniques for complex systems. Chapter 3 describes the data source, the geographical unit used in this study, and the areal interpolation methodology. Chapter 4 focuses on the identification of the rules, the extraction of the variables and the building of the coupled system dynamics. Chapter 5 introduces the neighborhood effects, and the calibration of the simulation. Chapter 6 assesses the resilience and the sustainability of the land cells. Chapter 7 defines the Relative Land Price concept to measure a relative value of the land cells due to their population and "utility" (to be defined later), according to the simulated results. Chapter 8 compares the simulated results between the "north" part and the "south" part partitioned by the hypothesized north-south boundary. Chapter 9 summarizes the conclusions and the significant findings. 


\subsection{References}

Blum, M. D., Roberts H. H. (2009). Drowning of the Mississippi Delta due to insufficient sediment supply and global sea-level rise. Nature Geoscience Letters 2(7), 488-49.

Collins, S. L., Carpenter S. R., et al. (2011). An integrated conceptual framework for longterm social-ecological research. Frontiers in Ecology and the Environment 9(6), 351357.

CPRA, Coastal Protection and Restoration Authority of Louisiana, (2012). Louisiana's comprehensive master plan for a sustainable coast. CPRA, State of Louisiana, Baton Rouge, LA.

Kates, R. W. (2011). From the unity of nature to sustainability science: ideas and practice. CID Working Paper (218). Center for International Development, Harvard University. Cambridge, MA. http://www.hks.harvard.edu/centers/cid/publications/facultyworkingpapers/cid-working paperno.-218. Last access 11/15/2011.

Lam, N. S. N., Arenas H., Li Z., Liu K.B. (2009a). An estimate of population impacted by climate change along the U.S. coast. Journal of Coastal Research, Special Issue 56, 1522-1526.

Lam, N.S.N., Arenas H., Pace R.K., LeSage J.P., Campanella R. (2012a). Predictors of Business Return in New Orleans after Hurricane Katrina. PLoS One 7(10), e47935: 18.

Lam, N. S. N., Pace K., Campanella R., LeSage J., Arenas H., (2009b). Business return in New Orleans: decision making amid post-Katrina uncertainty. Public Library of Science (PLoS ONE), 4(8), 6765.

Lam, N.S.N., Liu, K.B., Reams, M., Rivera-Monroy, V., Xu, J., Pace, K., Dismukes, D. (2012b). CNH: Coupled natural-human dynamics in a vulnerable coastal system. NSF award abstract (http://nsf.gov/awardsearch/showAward?AWD_ID=1212112).

Lam, N., Reams, M., Li, K., Li, C., Mata, L. (2015a). Measuring community resilience to coastal hazards along the northern Gulf of Mexico. Natural Hazards Review.

Lam N.S.N., Qiang, Y., Arenas, H, Brito P., Liu, K.B. (2015b). Mapping and assessing coastal resilience in the Caribbean region. Cartography and Geographic Information Science 42(4): 315-322.

LeSage, J. P., Pace R. K., Lam N. S. N., Campanella R., Liu X. (2011a). New Orleans business recovery in the aftermath of Hurricane Katrina. Journal of Royal Statistics Society A 174(4), 1007-1027.

LeSage, J.P., Pace R.K., Lam N.S.N., Campanella R. (2011b). Space-time modeling of natural disaster impacts. Journal of Economic and Social Measurement 36:169-191. 
LeSage J.P., Pace R.K., Lam N.S.N., Campanella R, Liu X. (2011c). Do what the neighbors do: reopening businesses after Hurricane Katrina. Significance 8(4) 160-163

Li, C. (2013). Community Resilience to Coastal Hazards: An Analysis of Two Geographical scales in Louisiana. Master's Thesis. Baton Rouge, Louisiana: Louisiana State University.

Li, K. (2011). Temporal changes of coastal community resilience in the Gulf of Mexico Region. Master's Thesis. Baton Rouge, Louisiana: Louisiana State University.

Li, K., Lam, N., Qiang, Y., Zou, L., Cai, H. (2015). A cyberinfrastructure for community resilience assessment and visualization. Cartography and Geographic Information Science 34-39.

Liu, J. G., Dietz T., et al. (2007a). Complexity of coupled human and natural systems. Science 317(5844): 1513-1516.

Liu, J. G., Dietz T., et al. (2007b). Coupled human and natural systems. Ambio 36(8), 639-649.

Plyer, A. (2013). Facts for Features: Hurricane Katrina Recovery. GNOCDC. Greater New Orleans Community Data Center, 30 Jan. 2013. Web. 3 May 2013. http://www.gnocdc.org/Factsforfeatures/HurricaneKatrinaRecovery/. 


\section{CHAPTER 2 : LITERATURE REVIEW AND CONCEPT FRAMEWORK DEVELOPMENT}

Before building the concept framework of the spatial dynamic model, several terms and concepts need to be clarified. First, it is necessary to understand the concepts of sustainability, vulnerability, and resilience through a solid literature review. Thus, the background of resilience and sustainability is reviewed in this chapter. Second, In order to build a reasonable coupled natural and human dynamics model, the major modeling approaches and techniques are also reviewed, and the advantages and verification methods for using these techniques were summarized. Third, the terminology used in this spatial dynamic model and the concept framework development are illustrated. This chapter serves as the theoretical foundation for the development of the proposed model.

\subsection{Coupled Natural and Human Dynamics and Complex Systems}

It has been widely recognized that to assess the resilience and the sustainability of a place-based system, one must consider both the natural and the human systems and evaluate how both systems are coupled (Bolin et al., 2000; Liu J. et al., 2007a\&b; Collins et al., 2010; Kates, 2011). The term coupled natural and human (CNH) system was evolved to describe a branch of interdisciplinary study that examines the interactions between ecological and social systems. A simple and straightforward definition is that $\mathrm{CNH}$ systems are integrated systems

in which human components interact with natural components (Liu J. et al., 2007a\&b). From the program solicitation of the dynamics of coupled natural and human systems by the National Science Foundation (NSF 14-60), a CNH dynamics research should include the following aspects: (1) the dynamics of a natural system; (2) the dynamics of a human system; (3) the processes through which the natural system affects the human system; and (4) the processes through which the human system affects the natural system. This study emphasizes the human dynamics part in a CNH system, and it develops a model mainly addressing the last three aspects. 
CHN systems are treated as complex adaptive systems (Levin, 1999; Gunderson \& Hollings, 2002), rather than as separate ecological and social systems in traditional studies. Although there is no general agreement on the definition of complex systems, they normally feature large population of interacting elements. They are "complex" because the elements are interacting in a disordered way with no central controller, and the emergences they generated are difficult to be anticipated by the traditional "top-down" approaches. Studies on $\mathrm{CNH}$ systems offer unique insights into complexities that cannot be gained from separate ecological or social research such as the reciprocal effects, the feedback loops, the nonlinearity, and the thresholds of shifting system status (Liu J. et al., 2007a\&b). There are many ways to manifest the complexity in a coupled system, including path-dependence, criticality, self-organization, difficulty of prediction, and emergence of qualities not analytically tractable from system components and their attributes alone (Sole \& Goodwin, 2000; Manson, 2001; Bankes, 2002).

\subsection{Vulnerability, Resilience, and Sustainability}

There is abundant literature on vulnerability, resilience, and sustainability (National Research Council NRC, 2012). Some researchers define resilience as the speed of a system returning to the original state after disruption, whereas others define resilience as the magnitude that a system could be perturbed without shifting to a different state (Holling, 1973, 1996; Walker et al., 2006a\&b). The two definitions reveal two different ways of assessing the resilience capability of a given system: either by measuring the speed of return or by measuring the threshold of changing. Both definitions imply that resilience is an ability of a system to fight against disturbances from the outside environment. Adger and others (2005: p.1036) defined resilience as "the capacity of linked social-ecological systems to absorb recurrent disturbances such as hurricanes or floods so as to retain essential structures, processes, and feedbacks".

The term vulnerability is closely related to resilience in the literature (Lam et al., 2015a). Folke and others (2002) considered vulnerability as "the propensity of an ecological 
system to suffer harm from exposure to external stresses and shocks". Turner and others (2003) considered vulnerability to include three dimensions: exposure to the hazard, sensitivity of the population to that exposure, and an ability to adapt to the changing circumstances. Cutter and others developed an index of social vulnerability, which is a measure of both the sensitivity of a population to natural hazards and its ability to respond to and recover from the impacts of hazards (Cutter \& Finch, 2008). The Intergovernmental Panel for Climate Change (IPCC) defined vulnerability as a function of exposure, sensitivity, and adaptive capacity (IPCC, 2001; Yusuf \& Francisco, 2009). In this study, we consider resilience a bigger concept that includes both aspects of vulnerability and adaptability. In terms of indicators representing vulnerability and adaptability, they are often overlapping with each other and used interchangeably (Cutter et al., 2003 \& 2010; Sherrieb et al., 2010; Community and Regional Resilience Institute (CARRI), 2013a\&b).

Sustainability is commonly defined as "the capacity of society to meet its current needs while assuring the wellbeing of future generations" (National Council on Science and the Environment [NCSE], 2013). Turner (2010) discussed the differences and commonalities between vulnerability and resilience and how they are related to sustainability science. He proposed the use of the concept of "tradeoffs" (among various sets of environmental services and human outcomes) as the key for intellectual fusion among the three concepts (vulnerability, resilience, and sustainability). Lately, resilience and sustainability are considered the new dual challenges to society (NCSE, 2013). In this dissertation, we adopt the position that resilience insures continuity, whereas sustainability insures balance. Resilience is a prerequisite of sustainability, and long-term resilience is sustainability (Lam at al., 2012b).

Despite the voluminous literature on resilience, vulnerability, and sustainability, there is no widely accepted index reflecting these concepts. The difficulties of resilience assessment arise because of the numerous definitions, and for those who have attempted to derive an index, there is seldom any validation. Developing metrics for measuring resilience is a priority (NRC, 2012). In this study, the assessment of resilience of a community will be based on the 
Resilience Inference Measurement (RIM) model, which was developed previously by our research group (Baker, 2009; Li K., 2011; Lam et al., 2015a\&b; Li et al., 2015). The RIM model creates a new way to measure community resilience and has both the properties of validation and inference. The RIM model considers three dimensions and two abilities (or disabilities). The three dimensions are: (1) the exposure to hazards (such as the number of times a community is hit by hurricanes or climate-related hazards), (2) the damage from exposure to hazards (such as property damages), and (3) the recovery (such as population return). The community's ability to minimize the damage at the time of the event is called vulnerability. Similarly, the community's ability to bounce back over time is called adaptability.

In the RIM model, four resilience states from the lowest to the highest resilience, susceptible, recovering, resistant, and usurper, are defined. A susceptible system is considered the least resilient, as it has high vulnerability and low adaptability. A recovering system has about the mean vulnerability and adaptability. A resistant system has low vulnerability and average adaptability, whereas a usurper system has low vulnerability but very high adaptability. K-means clustering method is used to identify the discrete states of resilience of each geographic unit, and discriminant analysis is used to test the socioeconomic and environmental variables that discriminate these states. The selected variables are used to develop the resilience assessment function.

\subsection{Population Changes and Residential Dynamics}

Literature on population changes and residential dynamics is huge. However, studies that model human population dynamics in the context of vulnerability, resilience, and sustainability are not common. The United Nations defined several types of human migration and relocation according to the motives: urbanization, family reunification, impelled or reluctant migration, labor migration, forced migration, return migration, student migration, chain migration, and seasonal migration (Claydon, 2012). Different studies took their different 
favorite factors, such as lifecycle, economic motivations, neighborhood facilities, environmental amenities, and/or housing quality (Lindberg et al. 1992; Nijkamp et al. 1993; Dokmeci \& Berkoz, 2000; Torrens, 2001; Yin \& Muller, 2007; Niedomysl, 2008). Studies on the simulation of residential dynamics can be traced back to the basic segregation model as early as in the 1970s (Schelling, 1971). Most of the recent studies used "bottom-up" approaches featuring ABM techniques. Benenson (1998) examined the inhabitants' behaviors using changing economic and cultural status as well as the properties of local and global environment; the study is among the earliest on residential dynamics using ABM. Kii and Doi (2005) proposed a multi-agent model for testing various development policies. Li and Liu (2008) incorporated the sustainability theory into ABM. Fontaine and Rounsevell (2009) integrated household life-cycle events in their HI-LIFE ABM. With a few exceptions (Schultz and Elliott, 2012), studies on modeling residential dynamics in the context of hazard, vulnerability, or resilience remain to be scarce.

There are many issues in applying "bottom-up" methods in modeling residential dynamics. A major problem is how to explicitly define the dynamics of the "bottom" spatial units using empirical information. Li and Liu (2007) used an ABM modeling approach and determined the parameters of their ABM according to multi-criteria evaluation techniques; however, their method assumes all the modeling units perform the same way as experts. Urban economic theory has been widely used (Fujita, 1989; Krugman, 1991; Anas \& Kim, 1996; Chen, 2012), which demonstrates that the formation of urban spatial structure is an endogenous process resulting from the interactions among individuals, assuming a monocentric study area, with population density, land value, and housing price declining with distance from the center (Anas et al, 1998; Parker and Filatova, 2008). Recent studies extend this urban economic theory by incorporating developers' decisions on open space amenities and spatial externalities (Cavailhes et al, 2004; Caruso et al, 2007; Irwin and Bockstael, 2002; Wu and Plantinga, 2003), and by extending the space into polycentric form (Fujita and Ogawa, 1982; Fujita and Thisse, 2002; Munroe, 2007). Some empirical statistical models also gain popularity in specifying the 
local special dynamics, such as discrete choice model that uses conditional logit regression equation to predict parameters (McFadden, 1978; Bruch and Mare, 2012; Bruch, 2014). This study used statistical analysis (Elastic Net) to extract the rules to develop the coupled spatial dynamics and applied them to the spatial units. Then evolutionary algorithms were used to optimize the neighborhood effects.

\subsection{Modeling Techniques of Complex Systems}

To understand the complexity in many theoretical (e.g., Epstein \& Axtell, 1996; Axelrod \& Cohen, 1999; Axtell et al., 2002) and empirical CNH studies (Benenson \& Torrens, 2004; Batty, 2005, 2007), two major modeling tools, cellular automata (CA) (Batty et al., 1994, 1997; Clarke \& Gaydos, 1998; Malanson et al., 2006a \& b, Qiang and Lam, 2015), and agentbased modeling (ABM), have been extensively employed. There is significant overlaps between the two approaches. Increasingly researchers combine both approaches to examine complex systems (Brown et al., 2006; Brown \& Xie, 2006; Bennett et al., 2011). There are indeed a variety of agent-based models developed by different researchers for different applications. However, despite recent attempts to develop protocols and ontology for $\mathrm{CNH}$ systems and sustainability modeling, cross-fertilization between the various models remain very difficult (An, 2011; Nyerges et al., 2014; An et al., 2014)."

Bazghandi (2012) summarized some advantages of using ABM over other modeling techniques in complex systems modeling: the ability to capture emergent phenomena, providing a natural description of a system, and flexibility. Although Bazghandi's comments were meant for ABMs, these benefits are actually shared by many "bottom-up" modeling approaches. In most "bottom-up" modeling approaches, the ability to reveal emergent phenomena is the key benefit. The unique advantage of capturing emergent phenomena is that researchers do not need to know the macro-pattern dynamics. Only the micro-rules that control local interactions are needed to be specified, and the macro-level results that emerge through the simulation of the specified micro-interactions can be measured. The bottom-up modeling 
approach gives researchers the opportunity to gain knowledge and understanding of emergent phenomena previously beyond their reaches. In many cases, a "bottom-up" model design is the most natural way for describing and simulating a system composed of "behavioral" entities, and makes the model more likely to be closer to reality. The flexibility of "bottom-up" modeling approaches can be observed in multiple ways. First, it is easy to increase or reduce the independent bottom-level "behavioral" entities. It is also easy to tune the complexity of the modeling units, by changing their behaviors, their ability to learn and evolve, and the rules of interactions. It is also flexible to change the levels of description and aggregation. The model can be changed to animate at different levels, including the aggregate modeling units, subgroups of modeling units, or individual modeling units, all of which can coexist in a complex system.

\subsubsection{Features of Cellular Automata}

In this study, the spatial dynamic model developed is like a cellular automata model in some aspects, because the spatial units are represented by cells and the updating rules of the spatial units' attributes are similar to cellular automation rules. A cellular automata model uses cells with different states as the smallest modeling units, and defines the rules for updating the states over time with the consideration of the neighborhood effects. In a cellular automation model, each cell has a state space, from which the cell can choose its state values. The states are controlled by a set of cellular automation rules. The cellular automation rules set the state of each cell at each time step, according to its previous state and its neighbor cells' previous states. In some cellular automata models (Boccara and Cheong, 1992, 1993), the cells can also have mobility with certain site-exchange rules defined. Figure 2.1 shows the structure of a cellular automata model. 


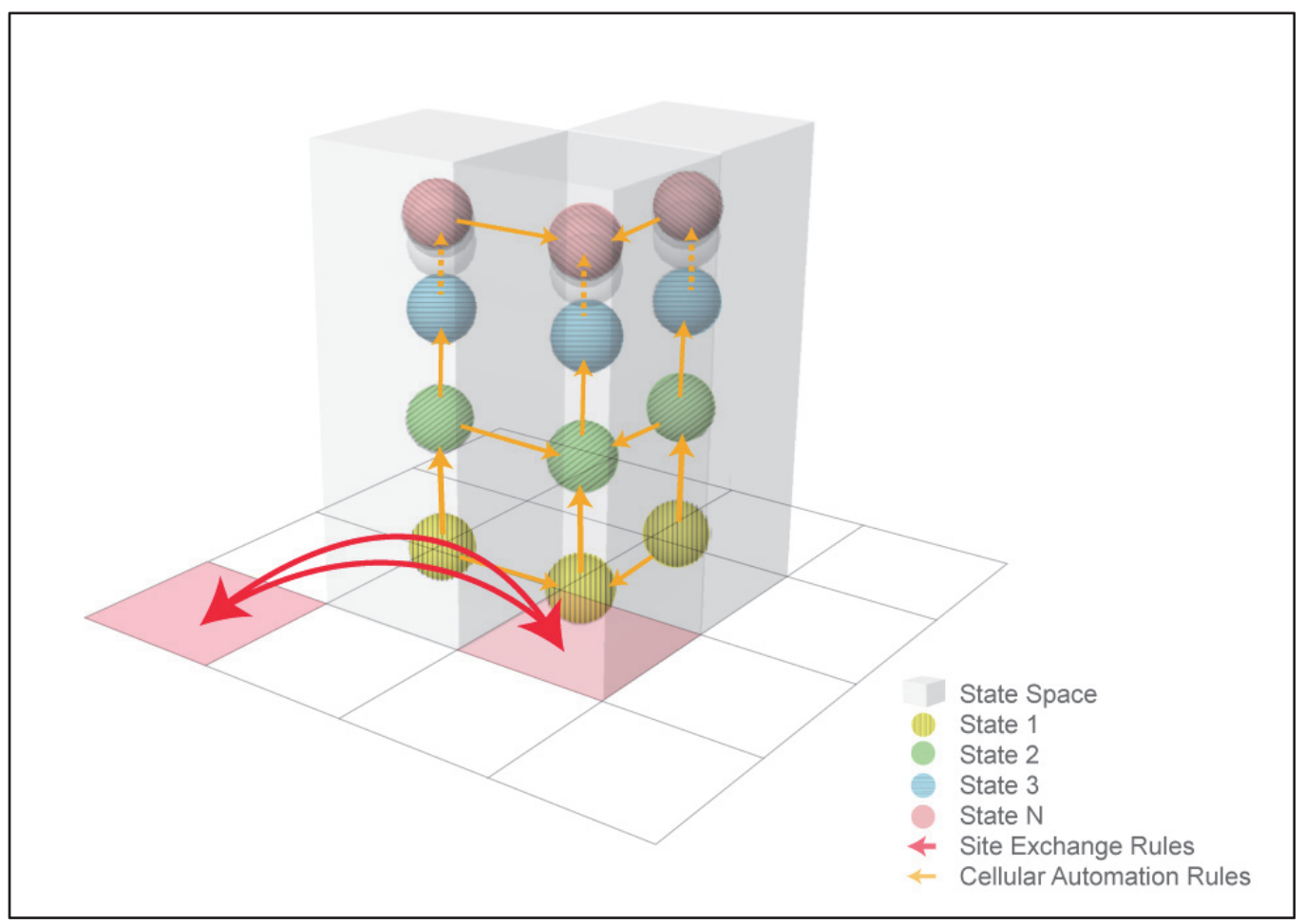

Figure 2.1 Structure of a Cellular Automata Model

The spatial units in this study are similar to the cellular automata in terms of representation by situated cells. However, different from the classic cellular automata which only have discrete states, the spatial units have a set of continuous attributes interacting with each other over time and space, and these interactions reflect the spatial units' capability of sensing the environments and acting on them.

\subsubsection{Features of Agent Based Modeling}

$\mathrm{ABM}$, as an approach to simulating the behavior of a complex system in which agents interact with each other and with their environment using local rules, has gained popularity and widespread use in many fields of study. ABM techniques have been used to study complex systems effectively, and have been proven to be fruitful in human system simulation (Monticino et al., 2007; Evans \& Kelly, 2004; Murray-Rust et al., 2013).

There is no precise definition with universal agreement of the term agent in the context of ABM (Macal and North, 2011). Some studies considered any type of independent component, either a software component or a sub-model, as an agent (Bonabeau 2001). Other 
researchers insisted that an agent's behavior must include adaptive components to represent the responses of the agents to the environment (Conte, 2002). Jennings (2000) from a computer science view emphasized the autonomous behavior of an agent an essential characteristic in an ABM. Figure 2.2 shows the basic structure of an ABM, which emphasizes some common points shared by these various definitions. These common characteristics of ABMs include that: (1) the agents have the ability to change their physical locations and modify their attributes, (2) the agents can actively sense the environment conditions and response accordingly, and (3) the agents can interact with other agents within their perspectives.

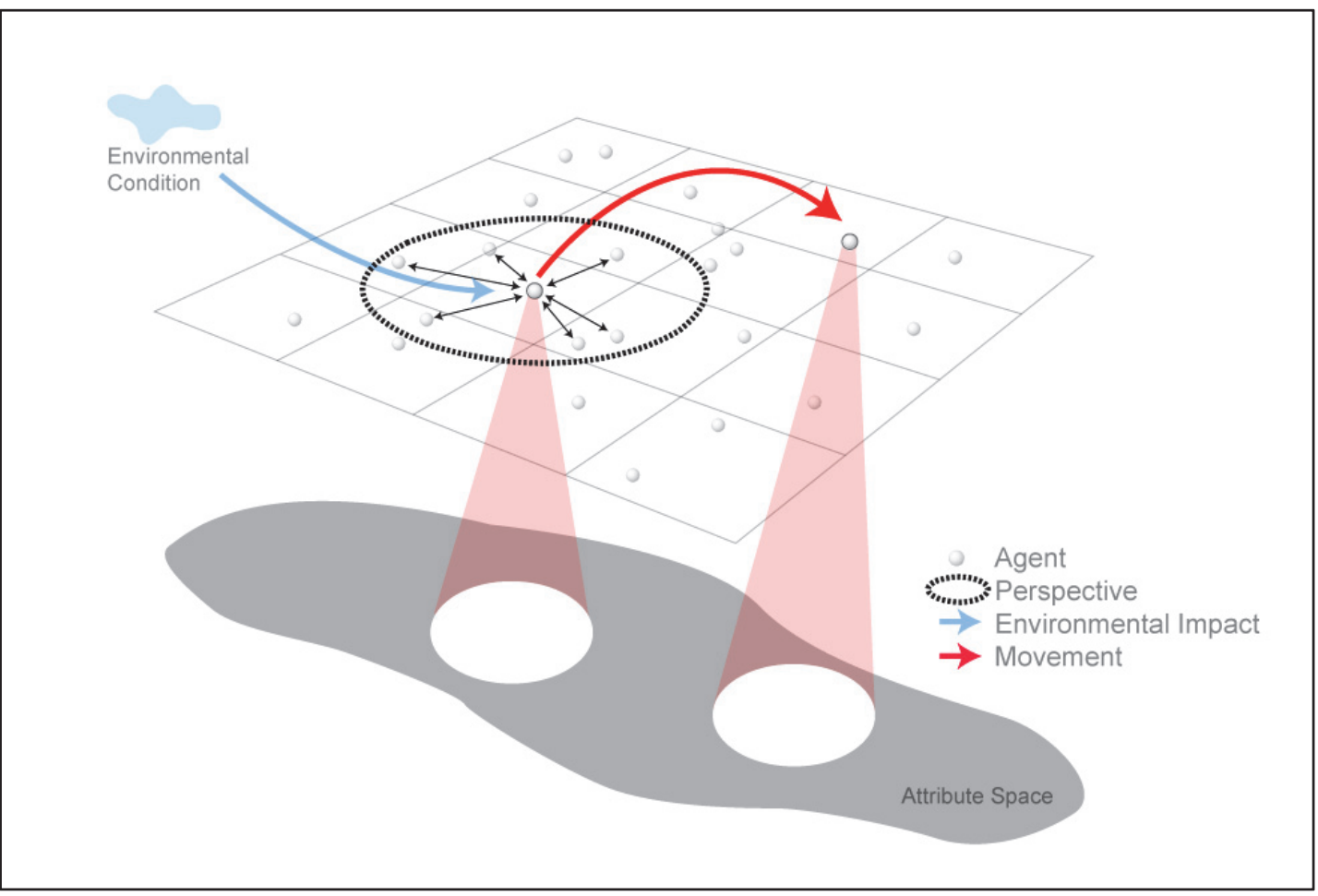

Figure 2.2 Structure of an Agent Based Model

Inspired by some of definitions of features of ABM (Wooldridge, 1995; Russel, 1995), three characteristics are considered as useful for my system dynamic model: autonomy, interaction ability, and reactivity. Autonomy means that units in the model can self-control their own internal states without interventions from other controllers. Interaction ability means that units in the model are able to interact with other units located within their perspectives. Reactivity means that units in the model are capable of perceiving their environment and 
responding. The spatial dynamic model developed in this study is not an ABM, however the developed model process these three features.

\subsection{Lagrangian V.S Eulerian}

In modeling the spatial dynamics of a system, two frameworks originally from the fluid dynamics modeling researches can be used: Lagrangian and Eulerian (Zhang and Chen, 2007). In a Lagrangian framework, the dynamics of the individuals are modeled by tracking their positions from places to places over time. In contrast, a Eulerian framework only focuses on the in-and-out flows constituted by the individuals to situated fields. For the modeling of the human dynamics in this study, a Lagrangian framework fits better in modeling the population migration from places to places, whereas a Eulerian framework is more suitable for modeling the population changes. Contraposing the spatial dynamic model in this study, a Eulerian framework was used to model the population changes, since the data for human migration is not available but only population changes data is available. Thus situated cells were used as the bottom-level spatial units in this model. Figure 2.3 shows of the differences between a Lagrangian Framework and a Eulerian Framework for modeling the spatial dynamics in this study. 


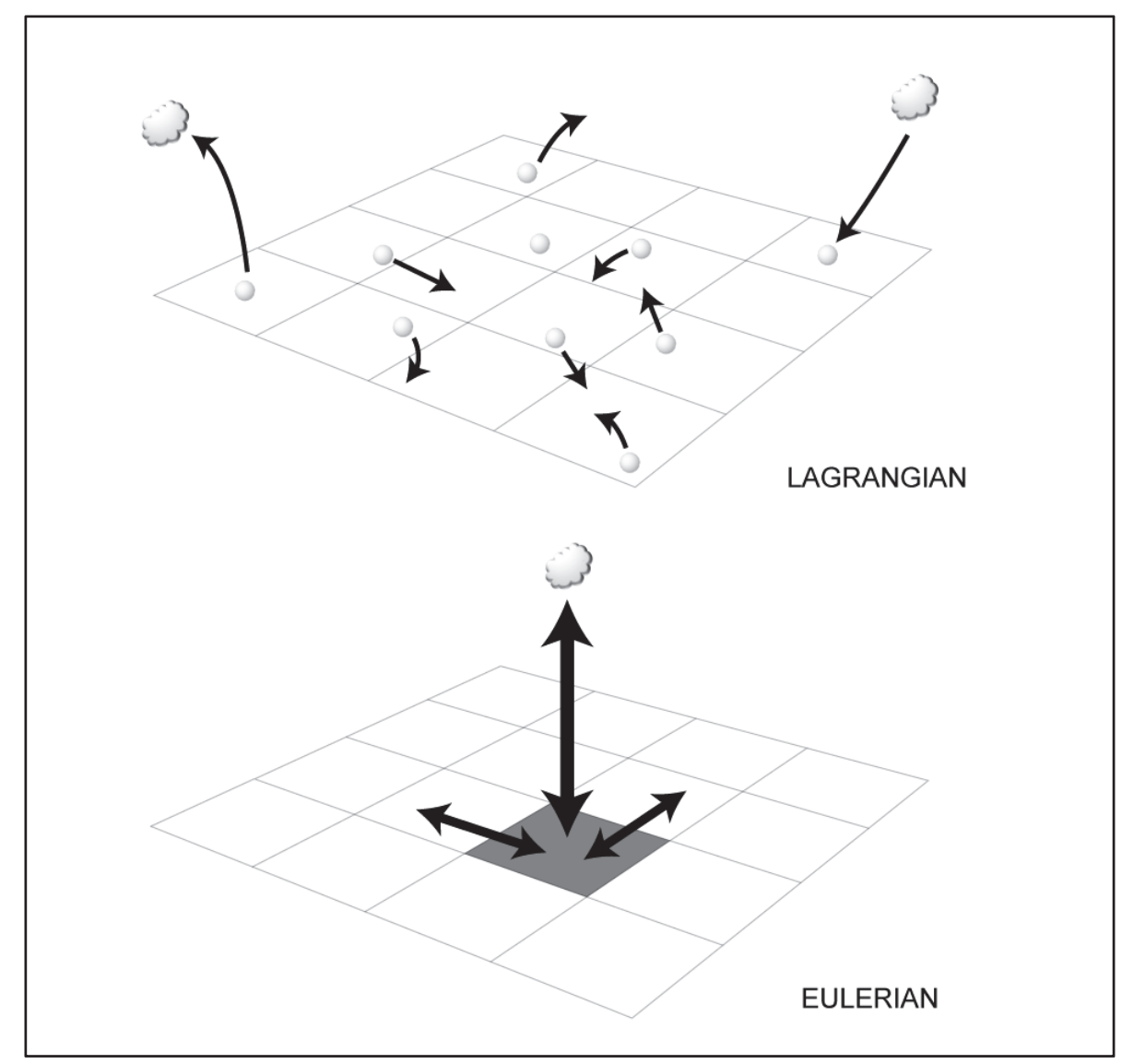

Figure 2.3 Lagrangian Framework V.S Eulerian Framework

In Figure 2.3, a cellular space is defined for modeling the dynamics of certain entities. A Lagrangian framework simulates the movements of the entities from places to places in the space with some adding-ins and moving-outs of the entities over time. In contrast, a Eulerian framework simulates the factors that influence the in-and-out flows consisting of the entities between each situated field and its neighbor fields or the external field out of the cellular space.

\subsection{Calibration and Genetic Algorithms}

When developing a complex system model consisting a lot of interacting units, the tuning of the model constitutes a crucial step of the design process. Indeed, a complex system model is generally characterized by lots of parameters, which together determine the global dynamics of the complex system. It typically have many parameters governing the behavior of the system, and using Genetic Algorithms (GA) is one of the choices to tune these parameters. GAs have been applied successfully to optimize these parameters, in many complex system 
models, most of which are ABMs (Calvez \& Hutzler, 2006; Heppenstall et al., 2007; Namboodiri, 2006; Wang \& Zhang 2012). The approaches common to all these previous studies are that they all consider the calibration and validation of a model as an optimization problem. The optimization function would be the difference between the simulated and the real systems. Genetic Algorithms are used to explore the parameter space and to find the best parameter set with respect to the optimization function.

Genetic algorithms are a family of computational models inspired by the evolution theory. In a GA's framework, the potential solution of a problem is encoded as a linear data structure, which is called a chromosome. The algorithm works on a set of chromosomes that is called a population. Operators are applied to this population. Each chromosome is then evaluated using a fitness function, which measures how good this potential solution is with respect to the initial problem. A selection is made among the population of chromosomes. Recombination and mutation operators are then applied to this population. The recombination consists in swapping parts between two chromosomes, which is the most frequent operator in genetic algorithms. The mutation consists of changing a part of a chromosome. The best solution will be driven from the last generation of population with the highest fitness function score.

\subsection{The Framework of the Spatial Dynamic Model}

A typical framework of a spatial dynamic model would include: the definition of the modeling units, the definition of the functions controlling the dynamics within each modeling unit, and the definition of the essential characteristics of the modeling units. In this study, the spatial dynamic model consists of the following components: a set of spatial units as the

modeling units, a set of finite internal states and their initials for the spatial units, a set of functions that determine a spatial unit's internal states and its actions over time (autonomy functions), a set of functions that determine the inputs from other spatial units and the outputs 
to them (interaction functions), and a set of functions determine a spatial unit's responses to the natural environment (reactivity functions).

Two major challenges in applying the "bottom-up" approaches to model the complex systems are: first, the typical large number of bottom-level entities can require sufficient computation time to limit analyses that use hundreds of runs, second and most importantly, the data for the individual entities are often only available in aggregative forms, such as at the county, the census tract, or the census block level for the human dynamics components. In order to build an empirical model for a relatively large geographic scale such as the Lower Mississippi River Basin in this study, modeling units defined in an aggregation form need to be used (Scheffer et al., 1995; Hellweger, 2008). In this study, spatial units immersed in a cellular space were designed in an attempt to capture the emergent phenomena of the coupled system dynamics, which are the outcomes of the social, economic and environment variables of these spatial units interacting over space and time. A spatial unit will be used as a coherent modeling unit of the analysis on the coupled human and natural dynamics. A spatial unit is an aggregation of the population with the homogeneous social and economic characteristics located in the same land area. The spatial units are situated entities located in a grid space, and a spatial unit is represented by a $3 \mathrm{~km}$ by $3 \mathrm{~km}$ grid lattice.

The internal states of a given spatial unit in this study are the number of population it contains and its socioeconomic characteristics (utility). Autonomy functions control the flows of the internal states. The spatial units' responses to their environment in this study are reflected by the changes of the developed land area percentage of the land area they locate in. Reactivity functions control the responses of the spatial units to their environment. It should be noted that with the consideration of neighborhood effects, the reactivity responses of a spatial unit not only change the developed land are percentage of the land area it locates in but also affect the changes of the developed land area percentage of its neighboring land areas. Interaction functions affect the spatial units' response actions using the outputs from its neighboring spatial units as inputs. The outputs of each spatial units are the changes of its internal states 
(population and utility), and these outputs are the inputs to its neighboring spatial units. Taking the internal state of population as an example, the spatial units actively use the autonomy functions to calculate their population changes at each time step and output them to their neighboring spatial units. For each spatial unit, the outputs from its neighbors are received by its interaction functions as input and response actions will be triggered. These actions will lead to population changes of this spatial unit in the next time step, hence its population changes are determined not only by its autonomy functions but also its interaction functions. The updating rules of the spatial dynamic model in this study are tightly bonded with these three sets of functions. The structure of the spatial units in this study are shown in Figure 2.4.

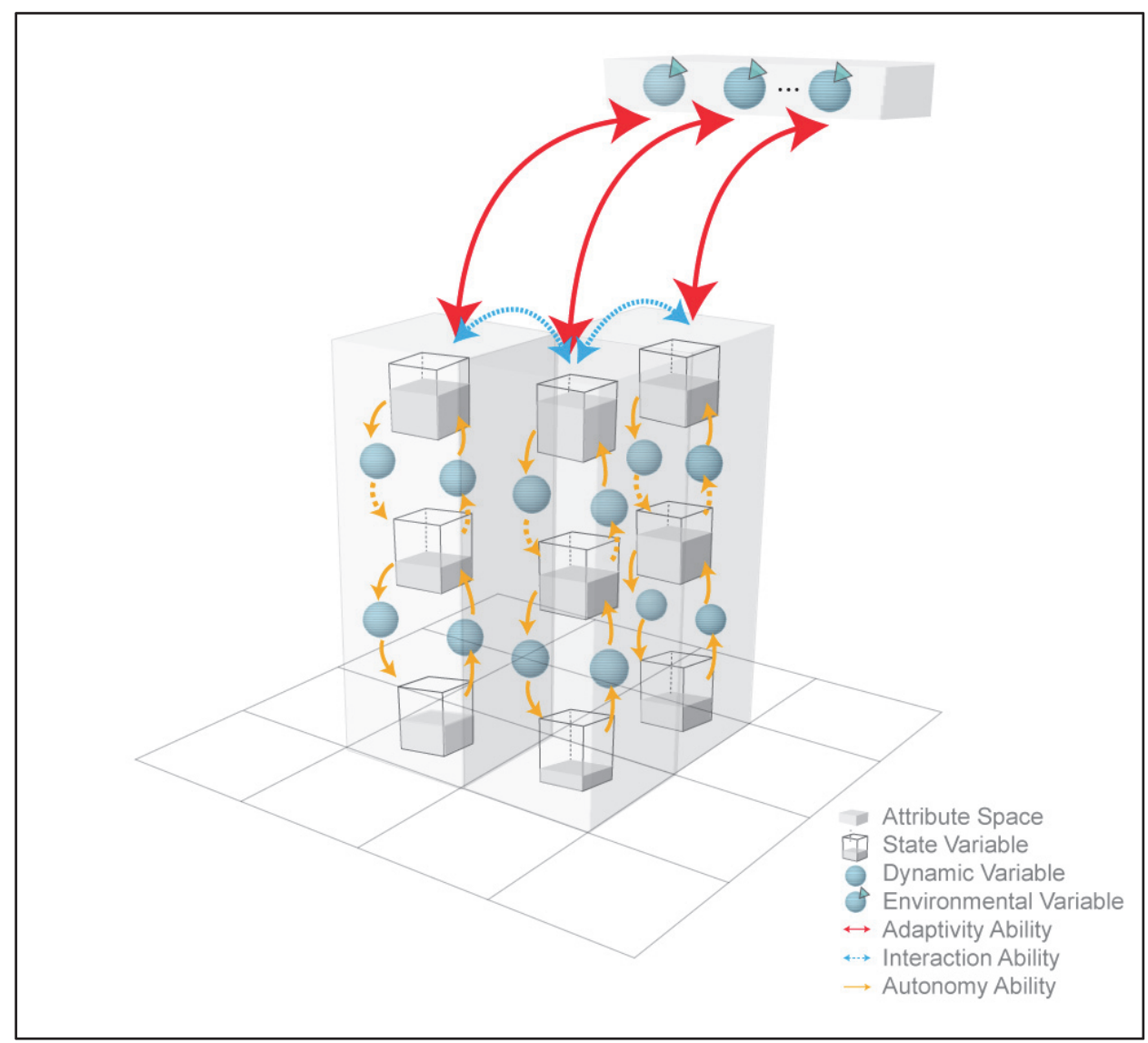

Figure 2.4 Structure of the Modeling Units of the Spatial Dynamic Model in this Study

In comparison with cellular automata, the spatial units in this study are similarly represented by the situated cells, but they have more than one continuous state variables, the changes of which are driven by the three sets of functions with several feedback loops. 
Borrowing form some ABMs, the three characteristics of the ABMs are reflected in this Eulerian model by the three sets of spatial dynamic functions of the spatial units: autonomy, interaction ability, and reactivity. The autonomous characteristic is reflected by that a spatial unit is able to control its internal state. Interaction ability is included because the spatial units are able to "communicate" with their neighbors. Finally, the fact that the spatial units are able to actively perceive their environment and react accordingly that leads to population changes reflects the reactivity.

\subsection{Development Platforms}

Although the spatial dynamic model built in this study was not an ABM, an ABM development platform was chosen, because most of the desired functions of the modeling units can be realized by utilizing the embedded libraries in an ABM development platform. The $\mathrm{ABM}$ development platforms are as diverse as the community of people who use them. With so many toolkits available, the choice of which one is best suited for a project is especially troublesome for researchers. Nikolai and Madey (2008) characterized each possible platform based on five important characteristics users consider when making choices, and then categorized their characteristics into user-friendly taxonomies, which offered a useful guide. The five characteristics they examined are: language required to program a model and to run a simulation, operating system required to run the toolkit, type of license that governs the toolkit, primary domain for which the toolkit is intended, and types of support available to the user.

Various ABM tool kits such as Swarm (http://www.swarm.org), Repast (http://repast.sourceforge.net), Net Logo (http://ccl.northwestern.edu/netlogo), TerraME (http://www.terrame.org), GAMA (https://github.com/gama-platform), MASON (http://cs.gmu.edu/ eclab/projects/mason), and AnyLogic (http://www.anylogic.com) have been chosen and used by various researchers. Each one of those have different capabilities such as ease of GIS integration and the level of programming expertise required. Various surveys give a better understanding of the abilities of these modelling platforms (Nikolai and 
Madey, 2008; Railsback et al., 2006). AnyLogic was chosen because of its proven capabilities to support spatial simulations and the extensive user support available through its website. 


\subsection{References}

Adger, W.N., Hughes T.P., Folke C., Carpenter S.R., Rockstrom J. (2005). Social-ecological resilience to coastal disasters. Science 309:1036-1039.

An, L. (2011). Modeling human decisions in coupled human and natural systems: Review of agent-based models. Ecological Modeling 229:25-36.

An, L., Zvoleff, A., Liu, J., Axinn, W. (2014) Agent-based modeling in coupled human and natural systems (CHANS): Lessons from a comparative analysis. Annals of the Association of American Geographers 104(4):723-745.

Anas, A., Kim, I. (1996). General equilibrium models of polycentric urban land use with endogenous congestion and job agglomeration. Journal of Urban Economics 40(2): 232-256.

Axelrod, R., Cohen, M.D. (1999). Harnessing Complexity: Organizational Implications of a Scientific Frontier. The Free Press, New York.

Axtell, R.L., Epstein, J.M., Dean, J.S., Gumerman, G., Swedlund, A., Harburger, J. (2002). Population growth and collapse in a multi-agent model of the Kayenta Anasazi in Long House Valley. Proceedings of the National Academy of Sciences 99:7275-7279.

Baker, A. (2009). Creating an Empirically Derived Community Resilience Index of the Gulf of Mexico Region. Master's thesis. Baton Rouge, Louisiana: Louisiana State University.

Bankes, S.C. (2002). Tools and techniques for developing policies for complex and uncertain systems. Proceedings of the National Academy of Sciences, 99: 7263-7266.

Batty, M. (2005). Agents, cells, and cities: new representational models for simulating multiscale urban dynamics. Environment and Planning A 37:1373-1394.

Batty, M. (2007). Cities and Complexity: Understanding Cities with Cellular Automata, AgentBased Models, and Fractals. The MIT Press, Cambridge, Massachusetts.

Batty, M., Couclelis H., Eichen M. (1997). Urban systems as cellular automata. Environment and Planning $B$ 24:59-164.

Batty, M., Xie Y., Sun Z. L. (1994). Modeling urban dynamics through GIS-based cellular automata. Computer, Environment and Urban Systems 23:205-233.

Bazghandi, A. (2012). Techniques, advantages and problems of agent based modeling for traffic simulation. International Journal of Computer Science Issues 9(1), 115-119.

Benenson, I. (1998). Multi-agent simulations of residential dynamics in the city. Computers, Environment and Urban Systems 22(1): 25-42. 
Benenson, I., Torrens, P.M., (2004). Geosimulation: Automata-Based Modelling of Urban Phenomena. Wiley, West Sussex, UK.

Bennett, D., Tang, W., and Wang, S. (2011). Toward an understanding of provenance in complex land use dynamics. Journal of Land Use Science 6(2-3):211-230.

Bolin, B., Clark W., Corell R., Dickson N., Faucheux S., Gallopan G., Gruebler A., Hall M., Huntley B., Jaeger J., Jaeger C., Jodha N., Kasperson R., Kates R., Lowe I., Mabogunje A., Matson P., McCarthy J., Mooney H., Moore B., O'Riordan T., Schelnhuber J., Svedin U. (2000). Core Questions of Science and Technology for Sustainability. http://sustainabilityscience.org/content.html?contentid=776/. Last access 2/11/2014.

Bonabeau, E. (2002). Agent-based modeling: methods and techniques for simulating human systems, Proceedings of the National Academy of Sciences, 7280-7287.

Brown, D. G., Xie, Y. (2006). Spatial agent-based modeling. Guest Editorial. International Journal of Geographical Information Science 20(9):941-943.

Brown, D.G., Aspinall, R., Bennett, D.A. (2006). Landscape models and explanation in landscape ecology. The Professional Geographer 58(4):369-382.

Bruch, E. (2014). How population structure shapes neighborhood segregation. NIH Public Access 119(5): 1221-1278.

Bruch, E., Mare, R. (2012). Methodological issues in the analysis of residential preferences, residential mobility and neighborhood change. Sociological Methodology 42(1): 103154.

Calvez, B., Hutzler G. (2006). Automatic tuning of agent-based models using genetic algorithms. Lecture Notes in Computer Science 3891:41-57.

Caruso, G., Peeters D., Cavailhes J., Rounsevell M. (2007). Spatial configurations in a periurban city. A cellular automata based microeconomic model. Regional Science and Urban Economics 37:542-567.

Cavailhès, J., Frankhauser, P., Peeters, D., Thomas, I. (2004). Where Alonso meets Sierpinski: an urban economic model of a fractal metropolitan area. Environment and Planning $A$ 36:1471-1498.

Clarke, K.C., Gaydos L. (1998). Loose coupling a cellular automaton model and GIS: longterm growth prediction for San Francisco and Washington/Baltimore. International Journal of Geographical Information Science 12: 699-714.

Claydon, K. J. (2012). A global model of human migration. UCL Working Paper Series (186). CASA University College London. 
Chen, Y., Li, X., Wang, S., Liu, X. (2012). Defining agents' behavior based on urban economic theory to simulate complex urban residential dynamics. International Journal of Geographical Information Science 26: 1155-1172.

Collins, S. L., Carpenter S. R., et al. (2011). An integrated conceptual framework for longterm social-ecological research. Frontiers in Ecology and the Environment, 9(6), 351357.

Community and Regional Resilience Institute (CARRI). (2013a). Definitions of community resilience: an analysis. http://www.resilientus.org/wpcontent/uploads/2013/08/definitions-of-community-resilience.pdf $\quad$ (accessed 01/11/2014).

Community and Regional Resilience Institute (CARRI). (2013b). Building resilience in America's communities: observations and implications of the CRS pilots. A CARRI Report. $\quad$ http://www.resilientus.org/wp-content/uploads/2013/05/CRS-FinalReport.pdf (accessed July 3, 2013).

Conte, R. (2002). Agent-based modeling for understanding social intelligence, Proceedings of the National Academy of Sciences of the United States of America PNAS, 99(3): 71897190.

Cutter, S. L., Finch C. (2008). Temporal and spatial changes in social vulnerability to natural hazards. Proceedings of the National Academy of Sciences 105(7) 2301-2306.

Cutter, S. L., Boruff B. J., Shirley W. L. (2003). Social vulnerability to environmental hazards. Social Science Quarterly 84:242-261.

Cutter, S.L., Burton C. G., Emrich C. T., (2010). Disaster resilience indicators for benchmarking baseline conditions. Journal of Homeland Security and Emergency Management 7(1), Article 51.

Dokmeci, V., Berkoz L. (2000). Residential-location preferences according to demographic characteristics in Istanbul. Landscape and Urban Planning 48(1-2):45-55.

Epstein, J.M., Axtell R. (1996). Growing artificial societies: social science from the bottom up. Brookings Institute, Washington.

Evans, T.P., Kelly, H. (2004). Multi-scale analysis of a household level agent-based model of land cover change. Journal of Environmental management 72: 57-72.

Folke, C., Carpenter S., Elmqvist T., Gunderson L., Holling C.S., Walker B. (2002). Resilience and sustainable development: building adaptive capacity in a world of transformations. Ambio 31: 437-440.

Fontaine, C. M., Rounsevell M. D. A. (2009). An agent-based approach to model future residential pressure on a regional landscape. Landscape Ecology 24(9):1237-1254. 
Fujita, M. (1989). Urban economic theory: land use and city size. Cambridge: Cambridge University Press.

Fujita, M., Ogawa, H. (1982). Multiple equilibria and structural transition of non-monocentric urban configurations. Regional Science and Urban Economics 12: 161-196.

Fujita, M., Thisse, J.F. (2002). Economics of agglomeration: cities, industrial location and regional growth Cambridge: Cambridge University Press.

Hellweger, F. (2008). Spatially explicit individual-based modeling using a fixed superindividual density, Computers \& Geosciences, 144-152.

Heppenstall, A., Evens A. J., Birkin M. H. (2007). Genetic algorithm optimization of an agentbased model for simulating a retail market. Environment and Planning B: Planning and Design 34: 1051-1070.

Gunderson, L.H., Holling C.S. (2002). Panarchy: Understanding Transformations in Human and Natural Systems. Island Press, Washington, DC.

Holling, C.S. (1973). Resilience and stability of ecological systems. Annual Review of Ecology and Systematics 4:1-23.

Holling, C.S. (1996). Engineering resilience versus ecological resilience. In Schelze P, eds. Engineering within Ecological Constraints (31-44). National Academy Press, Washington DC.

Irwin, E.G., Bockstael, N.E. (2002). Interacting agents, spatial externalities and the evolution of residential land use patterns. Journal of Economic Geography (2):31-54.

Jennings, N. (2000). On agent-based software engineering, Artificial Intelligence, 277-296.

Kates, R. W. (2011). From the unity of nature to sustainability science: ideas and practice. CID Working Paper (218). Center for International Development, Harvard University. Cambridge, MA. http://www.hks.harvard.edu/centers/cid/publications/facultyworkingpapers/cid-working paperno.-218. Last access 11/15/2011.

Kii, M., Doi, K. (2005). Multi-agent land use and transport model for the policy evaluation of a compact city. Environment and Planning B: Planning and Design 32(4): 485-504.

Krugman, P. (1991). Increasing returns and economic geography. Journal of political economy 99(3):483-499.

Lam, N., Reams, M., Li, K., Li, C., Mata, L. (2015a). Measuring community resilience to coastal hazards along the northern Gulf of Mexico. Natural Hazards Review.

Lam, N.S.N., Qiang, Y., Arenas, H, Brito P., Liu, K.B. (2015b). Mapping and assessing coastal resilience in the Caribbean region. Cartography and Geographic Information Science 42(4): 315-322. 
Levin, S.A. (1999). Fragile dominion: complexity and the commons. Perseus Publishing, Cambridge, MA.

Li, K. (2011). Temporal changes of coastal community resilience in the Gulf of Mexico Region. Master's Thesis. Baton Rouge, Louisiana: Louisiana State University.

Li, K., Lam, N., Qiang, Y., Zou, L., Cai, H. (2015). A cyberinfrastructure for community resilience assessment and visualization. Cartography and Geographic Information Science 34-39.

Li, X., Liu, X.P. (2007). Defining agents' behaviors to simulate complex residential development using multi-criteria evaluation. Journal of Environmental management 85(4): 1063-1075.

Li, X., Liu X.P. (2008). Embedding sustainable development strategies in agent-based models for use as a planning tool. International Journal of Geographical Information Science 22(1):21-45.

Lindberg, E., Hartig T., Garvill J., Garling T. (1992). Residential location preferences across the life-span. Journal of Environmental Psychology 12:187-198.

Liu, J. G., Dietz T., et al. (2007a). Complexity of coupled human and natural systems. Science 317(5844): 1513-1516.

Liu, J. G., Dietz T., et al. (2007b). Coupled human and natural systems. Ambio 36(8): 639-649.

Macal, C., North, M. (2011). Introductory tutorial: Agent-based modeling and simulation, Proceedings of the 2011 Winter Simulation Conference (WSC).

Malanson, G.P., Zeng Y., Walsh S.J., (2006a). Complexity at advancing ecotones and frontiers. Environment and Planning A, 38, 619-632.

Malanson, G.P., Zeng Y., Walsh S.J., (2006b). Landscape frontiers, geography frontiers: lessons to be learned. The Professional Geographer, 58, 383-396.

Manson, S.M. (2001). Simplifying complexity: a review of complexity theory. Geoforum, 32, 405-414.

McFadden, Daniel. (1978). Choice of residential location. Cowles Foundation Discussion Paper 477.

Monticino, M., Acevedo, M., Callicott, B., Cogdill, T., Lindquist, C. (2007). Coupled human and natural systems: a multi agent-based approach. Environmental Modeling \& Software 22:656-663.

Munroe, D. K. (2007). Exploring the determinants of spatial pattern in residential land markets: amenities and disamenities in Charlotte, NC, USA. Environment and Planning B: Planning and Design 34: 336-354. 
Murray-Rust, D., Rieser, V., Robinson, D.T., Milicic, V. (2013). Agent-based modelling of land use dynamics and residential quality of life for future scenarios. Environmental Modeling \& Software, 46, 75-89.

Namboodir, E. (2006). Using agent based modeling and genetic algorithms to understand and predict the behavior of complex environmental systems. (Master's Thesis). Retrieved from https://etd.ohiolink.edu/.

National Council on Science and the Environment (NCSE) workshop. (2013). Resilience and Sustainability. Organized by EPA office of Research \& Development.

National Research Council Report. (2012). Disaster Resilience: A national Imperative. Washington, DC: The National Academies Press.

National Science Foundation. (2014). Dynamics of Coupled Natural and Human Systems $(\mathrm{CNH})$, Program Solicitation NSF 14-60.

Niedomysl, T. (2008). Residential preferences for interregional migration in Sweden: demographic, socioeconomic, and geographical determinants. Environment and Planning A, 40, 1109 - 1131.

Nijkamp, P., Van Wissen L., Rima A., (1993). A household life cycle model for residential relocation behavior. Socioeconomic Planning Sciences, 27, 35-53.

Nikolai, C., Madey, G. (2008). Tools of the trade: A survey of various agent based modeling platforms. Journal of Artificial Societies and Social Simulation 12(2): 2.

Nyerges, T., Roderick, M., Prager, S., Bennett, D., Lam, N.S.N. (2014) Foundations of sustainability information representation theory: spatial-temporal dynamics of sustainable systems. International Journal of Geographic Information Science 28(5): 1165-1185.

Parker, D. C., Filatova, T. (2008). A conceptual design for a bilateral agent-based land market with heterogeneous economic agents. Computers, Environment and Urban Systems 32:454-463.

Railsback, S. F., Lytinen, S. L., Jackson S. K. (2006). Agent-based simulation platforms: review and development recommendations. Simulation 82(9): 609-623.

Russel, S. J., Norvig P. (1995). Artificial intelligence: a modern approach. Nova Jersey, Prentice Hall.

Reams, M.A., Lam N.S.N., Baker, A. (2012) Measuring capacity for resilience among coastal counties of the U.S. Northern Gulf of Mexico Region. American Journal of Climate Change 1:194-204. 
Scheffer, M., Baveco, J.M., DeAngelis, D.L., Rose, K.A., van Nes, E.H. (1995). Superindividuals a simple solution for modelling large populations on an individual basis. Ecological Modelling 80: 161-170.

Schelling, T. (1971). Dynamic models of segregation. Journal of Mathematical Sociology 1(2):143-186.

Schultz, J., Elliott, J. R. (2012). Natural disasters and local demographic change in the United States. Population Environment 34(3): 293-312.

Sherrieb, K., Norris F. H., Galea S. (2010). Measuring capacities for community resilience. Social Indicators Research 99: 227-247.

Shanthi, M., Rajan, E. G. (2012). Agent based cellular automata: a novel approach for modeling spatiotemporal growth processes, International Journal of Application or Innovation in Engineering \& Management (IJAIEM) 1(3): 56-61.

Sole, R., Goodwin B. (2000). Signs of Life: How Complexity Pervades Biology. New York: Basic Books.

Sudhira, H. S., Ramachandra, T. V., Wytzisk, A., Jeganathan, C. (2005). Framework for integration of agent-based and cellular automata models for dynamic Geospatial Simulations, Technical Report: 100.

Torrens, P.M. (2001). Can geocomputation save urban simulation? Throw some agents into the mixture, simmer, and wait. University College of London, Centre for Advanced Spatial Analysis, Working Paper series 32.

Turner, B.L. (2010). Vulnerability and resilience: Coalescing or paralleling approaches for sustainability science? Global Environmental Change 20: 570-576.

Turner, B.L., Kasperson R.E., Matson P.A., McCathy J.J., Corell R.W., Christensen L., Eckley N., Kasperson J.X., Luers A., Martello M.L., Polsky C., Pulsipher A., Schiller A. (2003). Science and technology for sustainable development special feature: A framework for vulnerability analysis in sustainability science. Proceedings of the National Academy of Sciences 100(14): 8074-8079.

Walker, B.H., Anderies J.M., Kinzing A.P., Ryan P. (2006a). Exploring resilience in socioecological systems through comparative studies and theory development: Introduction to the special issue. Ecology and Society 11(1): 12.

Walker, B.H., Gunderson L.H., Kinzing C., Folke S.R., Carpenter L., Schultz. (2006b). A handful of heuristics and some propositions for understanding resilience in socialecological systems. Ecology and Society 11(1): 13.

Wang, Z., Zhang J. (2012). Agent-Based Modeling and Genetic Algorithm Simulation for the Climate Game Problem. Mathematical Problems in Engineering 2012, 14. 
Wooldridge, M. J., Jennings, N. R. (1995). Intelligent agents: theory and practice, Knowledge Engineering Review 10(2).

Wu, J., Plantinga, A. J. (2003). The influence of public open space on urban spatial structure. Journal of Environmental Economics and Management 46: 288-309.

Yin, L., Muller B. (2007). Residential location and the biophysical environment: exurban development agents in a heterogeneous landscape. Environment and Planning $B$ Planning and Design 34, 279-295.

Yusuf, A.A., Francisco H. (2009). Climate change vulnerability mapping for Southeast Asia. Singapore: Economy and Environmental Program for Southeast Asia (EEPSEA).

Zhang, Z., Chen, Q. (2007). Comparison of the Eulerian and Lagrangian methods for predicting particle transport in enclosed spaces. Atmospheric Environment 5236-5248. 


\section{CHAPTER 3 : STUDY AREA AND DATA}

\subsection{Study Area}

The study area, broadly recognized as the Lower Mississippi River Basin (LMRB), is located in southeastern Louisiana and extends from the parishes (i.e. counties) north of Lake Pontchartrain to the Gulf coast (Fig. 1.1). It consists of 25 counties and two major metropolitan areas (New Orleans in the South and Baton Rouge in the North). In this research, Lake Pontchartrain is considered as an approximate boundary, where parishes north of the Lake are considered the "North", whereas parishes south of Lake Pontchartrain are labeled as the "South". According to the U.S. 2010 census statistics, the total population in the study area was $2,699,486$. During the past decade (2000-2010), average population increased by $3.26 \%$. Parishes in the North experienced very high population growth (e.g., Ascension Parish 39.9\% and Livingston Parish 39.4\%), whereas parishes in the South had high population decrease (e.g., St. Bernard Parish $-46.6 \%$ and Orleans Parish -29.1\%)(Lam et al., 2012). Some of the increase in population in the North has been documented to be from the South (Plyer, 2013). Many other factors were also cited as potential reasons (Crisp, 2014).

\subsection{Data Acquisition and Description}

Three sets of data were collected: (1) Natural hazards data were obtained from the "Storm Data and the Storm Events Database" maintained by the National Climate Data Center (NCDC) from the National Oceanic \& Atmospheric Administration (NOAA) (http://www1.ncdc.noaa.gov/pub/data/swdi/stormevents/). Five major types of coastal hazards were included in this study: coastal (which includes coastal flooding and storm surge), flood, hurricane, thunderstorm, and tornado. The detail definitions of these hazard types are listed in Table 3.1. The locations of these hazards data come in three formats: counties or zones (a combination of several counties), metropolitan areas, or point locations with longitudes and latitudes. 
Table 3.1 Major Types of Coastal Hazards in this Study with Descriptions

\begin{tabular}{|c|c|c|}
\hline \multicolumn{2}{|c|}{ Types of Hazard Events } & Description of the Hazard Events (Definition By NOAA-NCDC) \\
\hline \multirow[b]{2}{*}{ Coastal } & Flood & $\begin{array}{l}\text { Flooding of coastal areas due to the vertical rise above normal water level caused } \\
\text { by strong, persistent onshore wind, high astronomical tide, and/or low } \\
\text { atmospheric pressure. }\end{array}$ \\
\hline & $\begin{array}{l}\text { Storm } \\
\text { Surge/Tide }\end{array}$ & $\begin{array}{l}\text { For coastal and lakeshore areas, the vertical rise above normal water level } \\
\text { associated with a storm of tropical origin (e.g., hurricane, typhoon, or tropical } \\
\text { storm) caused by any combination of strong, persistent onshore wind, high } \\
\text { astronomical tide and low atmospheric pressure. }\end{array}$ \\
\hline \multicolumn{2}{|l|}{ Flood } & $\begin{array}{l}\text { Any high flow, overflow, or inundation by water which causes or threatens } \\
\text { damage. }\end{array}$ \\
\hline \multicolumn{2}{|c|}{ Hurricane/Typhoon } & $\begin{array}{l}\text { A tropical cyclone in which the maximum 1-minute sustained surface wind is } 64 \\
\text { knots }(74 \mathrm{mph}) \text { or greater. }\end{array}$ \\
\hline \multicolumn{2}{|c|}{$\begin{array}{l}\text { Thunderstorm Wind } \\
\text { (Severe) }\end{array}$} & $\begin{array}{l}\text { Winds, arising from convection (occurring within } 30 \text { minutes of lightning being } \\
\text { observed or detected), Severe Thunderstorms have speeds of at least } 50 \text { knots ( } 58 \\
\text { mph). }\end{array}$ \\
\hline \multicolumn{2}{|c|}{ Tornado } & $\begin{array}{l}\text { A violently rotating column of air, extending to or from a cumuliform cloud or } \\
\text { underneath a cumuliform cloud, to the ground, and often (but not always) visible } \\
\text { as a condensation funnel. }\end{array}$ \\
\hline
\end{tabular}

(2) Demographic and socioeconomic data were obtained from the U.S. Census Bureau (http://www.census.gov/). Population count data was obtained at the census block level, whereas the other social and economic variables were obtained at the census tracts level. (3) Environmental data, including elevation, water body locations, and road transportation networks were obtained from the Nation Map View developed by the U.S. Geological Survey (USGS) (http://viewer.nationalmap.gov/viewer/). (4) The land cover and land use data were obtained from the Multi-Resolution Land Characteristics Consortium (MRLC) (http://www.mrlc.gov/). (5) Subsidence Rates data were from the NOAA Technical Report 50: Rates of Vertical Displacement at Benchmarks in the Lower Mississippi Valley and the Northern Gulf Coast (http://www.ngs.noaa.gov/heightmod/Tech50.shtml). (6) Energy structures data such as pipeline and oil/gas wells locations were from the Louisiana Department of Natural Resources (LDNR) Oracle database (http://sonris.com/dataaccess.asp). All the variables collected for the interpolation and the model building are listed in Table 3.2 and Table 3.3 . 
Table 3.2 Acronyms and Descriptions of the Socioeconomic and Housing Variables used in this Study.

\begin{tabular}{|c|c|c|c|}
\hline \multicolumn{4}{|c|}{ Socioeconomic and Housing Variables } \\
\hline Categories & Acronym & Description & Year \\
\hline \multirow{11}{*}{ Housing } & Occupied & Percent; Occupied Housing Units & 2000,2010 \\
\hline & NonVehicle & $\begin{array}{l}\text { Percent; Occupied Housing Units with No Vehicles } \\
\text { Available }\end{array}$ & 2000,2010 \\
\hline & NonFuel & Percent; Occupied Housing Units with No House Heating & 2000,2010 \\
\hline & NonPlumb & Percent; Occupied Housing Units Lacking Complete & 2000,2010 \\
\hline & NonKitchen & Percent; Occupied Housing Units Lacking complete Kitchen & 2000,2010 \\
\hline & NonTele & Percent; Occupied Housing Units with No Telephone & 2000,2010 \\
\hline & I & Service $\ldots$ & \\
\hline & NonMtg & Percent; Specified Owner Occupied Units without Mortgage & 2000,2010 \\
\hline & OwnerR & Percent; Owner Occupied Housing Units & 2000,2010 \\
\hline & MedValue & $\begin{array}{l}\text { Number; Median Value of Specified Owner Occupied Units } \\
\text { (Dollars) }\end{array}$ & 2000,2010 \\
\hline & MedRent & $\begin{array}{l}\text { Number; Median Gross Rent of Specified Renter Occupied } \\
\text { Units (Dollars) }\end{array}$ & 2000,2010 \\
\hline \multirow{9}{*}{ Households } & OCST20 & $\begin{array}{l}\text { Percent; Owner Cost as a Percentage of Household Income } \\
\text { Less than } 15 \text { Percent }\end{array}$ & 2000,2010 \\
\hline & OCST35 & Percent; Owner Cost as a Percentage of Household Income & 2000,2010 \\
\hline & Rent15 & Percent; Gross Rent as a Percentage of Household Income & 2000,2010 \\
\hline & Rent35 & $\begin{array}{l}\text { Percent; Gross Rent as a Percentage of Household Income } \\
\text { More than } 35 \text { Percent }\end{array}$ & 2000,2010 \\
\hline & OCSTWMTG & $\begin{array}{l}\text { Number; Median Selected Monthly Owner Costs with a } \\
\text { Mortgage (Dollars) }\end{array}$ & 2000,2010 \\
\hline & OCSTNMRG & $\begin{array}{l}\text { Number; Median Selected Monthly Owner Costs without a } \\
\text { Mortgage (Dollars) }\end{array}$ & 2000,2010 \\
\hline & HhSize & Number; Average Household Size & 2000,2010 \\
\hline & MeanTime & Number; Mean Travel Time to Work (Minutes) & 2000,2010 \\
\hline & MedIcm & Number; Households Median Income (Dollars) & 2000,2010 \\
\hline
\end{tabular}


Table 3.3 Acronyms and Descriptions of the Demographic and Environmental Variables used in this Study.

\begin{tabular}{|c|c|c|c|}
\hline \multicolumn{4}{|c|}{ Demographic and Environmental Variables } \\
\hline Categories & Acronym & Description & Year \\
\hline \multirow{8}{*}{ Individuals } & Female & Percent; Total Female Population & 2000,2010 \\
\hline & Population & Number; Total Population & 2000,2010 \\
\hline & Under5 & Percent; Total Population under 5 Years Old & 2000,2010 \\
\hline & Over65 & Percent; Total population over 65 Years Old & 2000,2010 \\
\hline & HighSch & Percent; Population over 25 Years Old with High School & 2000,2010 \\
\hline & Married & $\begin{array}{l}\text { Percent; Population over } 15 \text { Years Old and Now Married } \\
\text { (Except Separated) }\end{array}$ & 2000,2010 \\
\hline & Employed & Percent; Population over 16 Years Old Employed & 2000,2010 \\
\hline & Poverty & Percent; Individuals below Poverty Level & 2000,2010 \\
\hline \multirow{3}{*}{ Structures } & Road & Number; Road Density & 2007 \\
\hline & Pipeline & Number; Pipeline Density & 2007 \\
\hline & GasWell & Number; Oil and Gas Injection Wells Density & 2007 \\
\hline \multirow{3}{*}{ Environmental } & Damages & Number; Property Damages in 2010 Inflation Rate (dollars) & 2000 to 2010 \\
\hline & Subsidence & $\begin{array}{l}\text { Number; Subsidence Rate Interpolated by Empirical Bayesian } \\
\text { Kriging using Bench Marks }\end{array}$ & 2004 \\
\hline & Elevation & $\begin{array}{l}\text { Number; Mean Elevation Calculated from LIDAR images } \\
\text { (Meters) }\end{array}$ & 2000 to 2010 \\
\hline \multirow{2}{*}{$\begin{array}{l}\text { Land Use and } \\
\text { Land Cover }\end{array}$} & Developed & Percent; Percent of Developed Land Use Area & 2001,2011 \\
\hline & Water & Percent; Percent of Open Water Land Use Area & 2001,2011 \\
\hline
\end{tabular}

\subsection{Data Preprocessing}

Since the spatial units of the simulation model are in grid form $(3 \mathrm{~km}$ by $3 \mathrm{~km})$ and the data acquired are in various forms and at various scales, areal interpolation is needed to integrate the disparate data into a single platform. For data defined in vector data form, such as roads, pipelines, and oil/gas wells, kernel densities were calculated before areal interpolation to convert them into a continuous surface. For the variable of property damages, the events collected at point locations and city area locations were summarized into the county they belong to. For the subsidence rate variable, point interpolation is needed to convert it from points to a continuous surface.

The variable of subsidence rate was interpolated using the Empirical Bayesian Kriging method. Classic Kriging is a geostatistical technique that uses a "semivariogram", a function of the distance separating two locations and their variance, to quantify the spatial dependence in the data. This semivariogram is then used to define the weights that determine the contribution of each observed data point to the prediction of new values at the un-sampled 
locations (Lam, 1983, 2009). The Empirical Bayesian Kriging (EBK) differs from the classical kriging methods by accounting for the error introduced by estimating the semivariogram model (Krivoruchko, 2011). For EBK, after the semivariagram model is built, a new value is simulated at each of the input data locations. A new semivariogram model is estimated from the simulated data. Then the Bayes' rule is used to calculate a weight for this semivariogram, which shows how likely the observed data can be generated from the semivariogram. The semivarigram generation process is iterated and creates a spectrum of semivariograms (Figure $3.1)$.

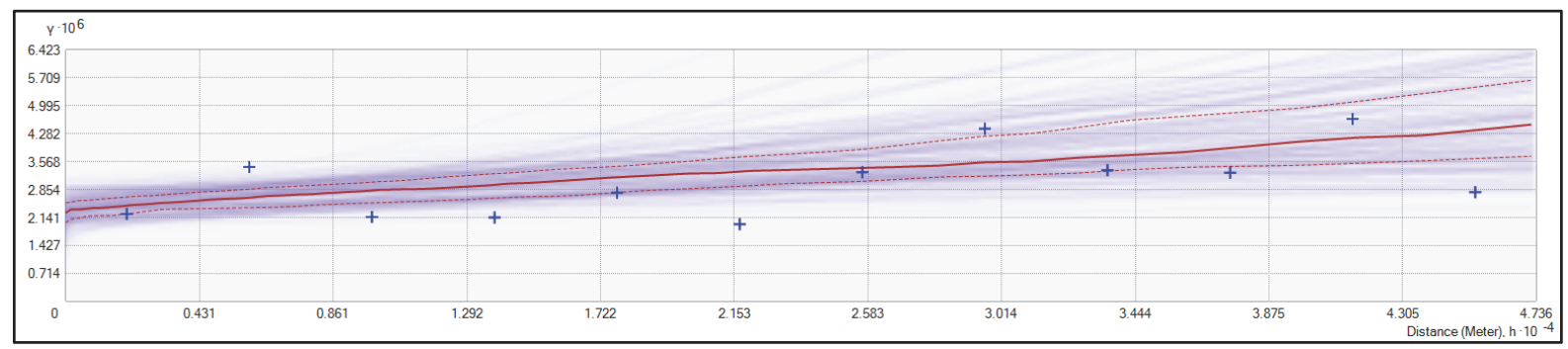

Figure 3.1 Spectrum of Semivarigrams of the Yearly Subsidence Rate in the Study Area

In Figure 3.1, the median of the distribution is shown with a solid red line. The $25_{\text {th }}$ and $75_{\text {th }}$ percentiles are colored with red dashed lines. The width of the blue lines is proportional to the semivariogram weights so that the models with smaller weights are shown by thinner lines. The final EBK predictions of the un-sampled locations for the average yearly subsidence rate are calculated using these weights.

\subsection{Areal Interpolation}

The data obtained in this study were from different temporal and spatial scales, such as subsidence data in point form, census data at the block, block-group, or census tract scale, and remote sensing images such as land use and land cover data in pixels. Even for the census data, especially at the block level, the geographic boundaries changed a lot over the study time span. For example, there were 62,716 blocks in the year 2000 , but the number increased to 94,609 in the year 2010. The number of census tracts were 654 in the year 2000, but became 683 in the year 2010. Thus, there is a crucial need to create a single platform that incorporates all 
these data from heterogeneous sources and units into a unified set of geographic spatial units to make them spatially and temporally comparable.

The term "areal interpolation" was first coined in Goodchild and Lam (1980) to denote the problem of transforming data defined in one set of areal units (source zones) to another (target zones), where the two sets of boundaries do not coincide (Lam 1980, 1983, 2009). The target zones for the areal interpolation in this study were the $3 \mathrm{~km}$ by $3 \mathrm{~km}$ contiguous cells. The $3 \mathrm{~km}$ by $3 \mathrm{~km}$ size was chosen with the consideration of a tradeoff between the resolution and the computational-time consumption later in the spatial dynamic model. Also the cell size is approximately equal to the average size of census tracts in urban area, the level at which most of our social and economic data were acquired. There are totally $5,8903 \mathrm{~km}$ by $3 \mathrm{~km}$ grids in the whole study area.

The areal interpolation procedure used in this study was an "intelligent" areal interpolation method (Cromley et al., 2012) that has the volume-preserving property. This study used the areal-weighting method with the developed land cover area as an ancillary layer. Ancillary data such as land cover in this study are called "control' variables. Areal interpolation with additional control variables is very similar to the principle of dasymetric mapping, a mapping technique designed to reflect within-zone variations (Lam, 1983, 2009).

With ancillary data, the accuracy of areal interpolation can be substantially improved. In this binary dasymetric method, control variables (land-cover data) were used to identify locations where certain attribute would not exist. In this study, it was assumed that only the developed area can have the values of the social and economic variables to be interpolated. Therefore, instead of using the total area of the source zone to derive the weights in areal weighting interpolation, only the developed area was used. For example, in estimating the population counts for the target zones ( $3 \mathrm{~km}$ by $3 \mathrm{~km}$ grids), if a source zone or a target zone has a large body of water area, it can be assumed that the water area does not have population. In summary, for a given target zone, its interpolated value is the weighted mean of the values of the source zones intersected with it, and the weights are defined by the total areas of these 
intersected areas on the ancillary layer. Figure 3.2 shows the areal interpolation process in this study with ancillary data.

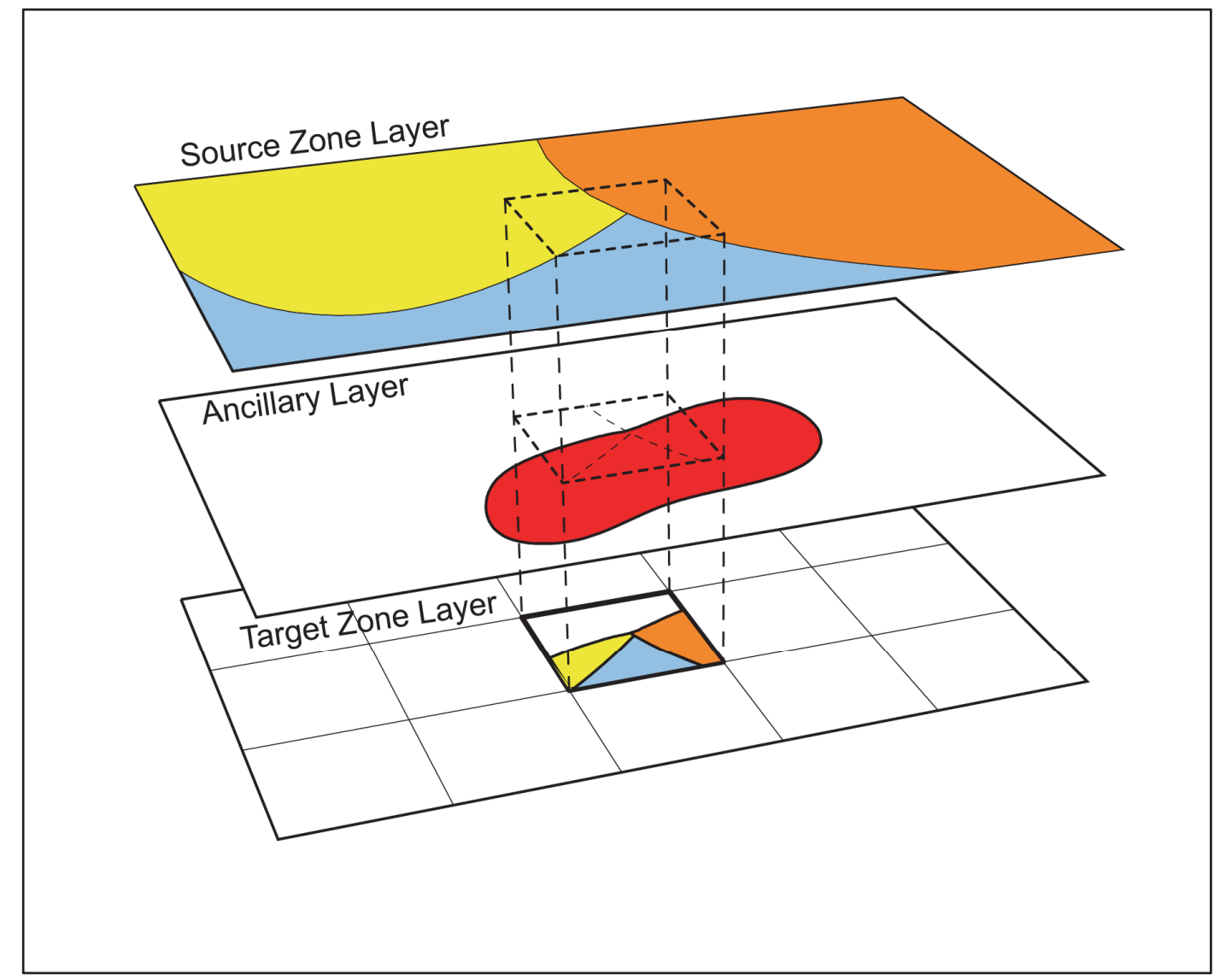

Figure 3.2 Areal Interpolation with Ancillary Layer in this study

There were two types of variables to be interpolated: "average rate" (intensive data such as median household income and percentage of individuals employed), and "total amount" (extensive data such as total population and total property damages). For the variables of the "average rate" type, a target zone's value was the mean of the values of the source zones, which the target zone intersects, weighted by the developed area. For the variable of the "total amount" type, the total number of developed land area pixels was calculated for each source zone. The value of a variable was divided by the total number of developed pixels in that source zone and assigned to the developed pixels in that source zone. Then the new target-zone value of this variable was simply the sum of the values of all developed cells within the target zone. Take the population density of 2000 (number of people per $9 \mathrm{~km}^{2}$ ) as an example, the values 
of the source zones (at the census block level), which means the values before the areal interpolation, are shown in Figure 3.3, and the values of the target zones (the $3 \mathrm{~km}$ by $3 \mathrm{~km}$ grids), which means the values after the areal interpolation, are shown in Figure 3.4. The comparisons of some other selected variables between before and after areal interpolation are shown in Appendix A.

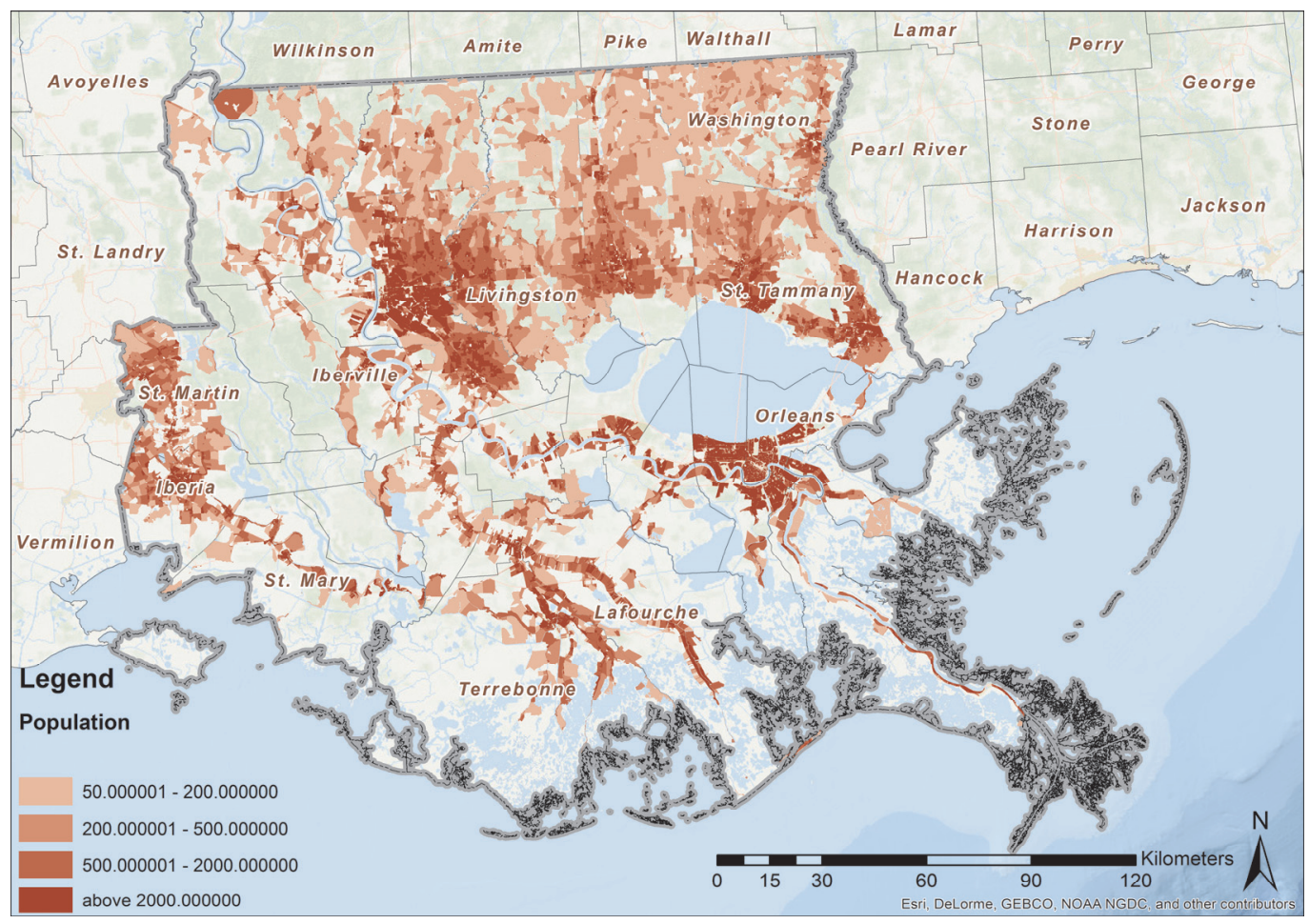

Figure 3.3 Population Density of the Census Blocks in 2000 


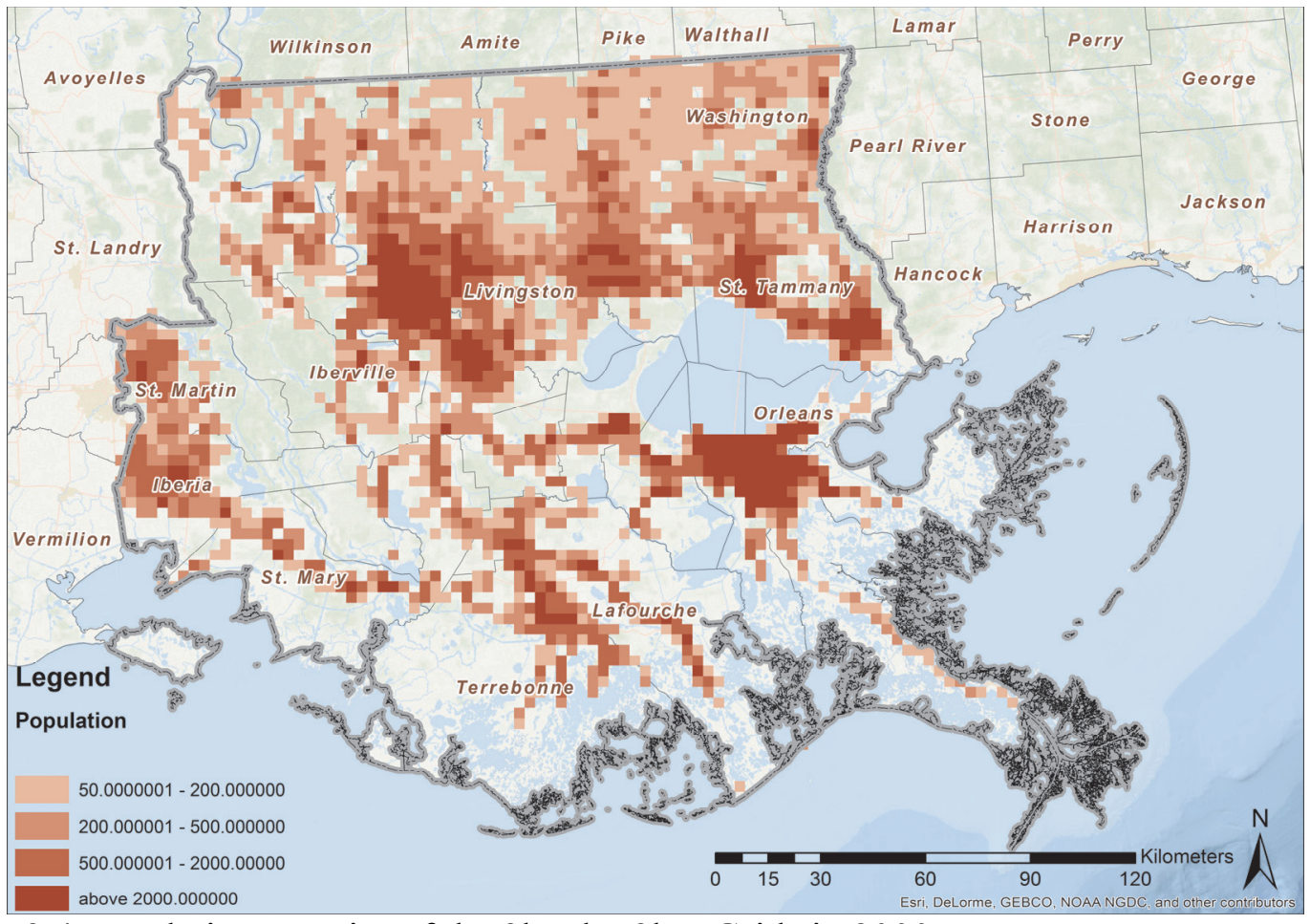

Figure 3.4 Population Density of the $3 \mathrm{~km}$ by $3 \mathrm{~km}$ Grids in 2000 


\subsection{References}

Crisp, E. (2014, February 7th). Census says EBR lost residence. The Advocate, $1 \mathrm{~b}$.

Cromley, R. G., Hanink D. M., Bentley, G. C. (2012). A quantile regression approach to areal interpolation. Annals of the Association of American Geographers 102(4):763-777.

Goodchild, M. F., Lam, N.S.N. (1980). Areal interpolation: a variant of the traditional spatial problem. Geo-Processing 1:297-312.

Krivoruchko, K. (2011). Spatial statistical data analysis for GIS users. Redlands, CA: ESRI Press, 928.

Lam, N.S.N. (1980). Methods and Problems of Areal Interpolation. PhD Dissertation, University of Western Ontario. London, Ontario, Canada.

Lam, N.S.N. (1983). "Spatial Interpolation Methods: A Review.” The American Cartographer 10(2):129-149.

Lam, N.S.N., Kitchin, R., Nigel, T. (2009). Spatial interpolation. In International Encyclopedia of Human Geography, Oxford: Elsevier 10: 369-376.

Lam, N.S.N., Liu, K.B., Reams, M., Rivera-Monroy, V., Xu, J., Pace, K., Dismukes, D. (2012). CNH: Coupled natural-human dynamics in a vulnerable coastal system. NSF award abstract.

Plyer, A. (2013). Facts for Features: Hurricane Katrina Recovery. GNOCDC. Greater New Orleans Community Data Center. 


\section{CHAPTER 4 : VARIABLE SELECTION AND RULES EXTRACTION}

The variable selection and rules extraction methods of the spatial dynamics model are described in this chapter. There are three stock variables in the system dynamics: population, developed land area percentage, and utility. The stock variable of utility is defined as a linear combination of the selected socio-ecological variables (details in Section 4.1.3). Elastic Net, a type of regulated linear regression approach, was used to select the variables, determine their coefficients, and derive the coupled relationships within the spatial dynamic model.

\subsection{Regulated Regression}

In order to derive the relationships to be applied to the spatial dynamic model, statistical learning methods that ensure the discovery of the most relevant predictive variables with a high prediction accuracy is needed. A possible way is to use the best subset selection approach, which conducts the ordinary regression on each possible subset of all the variables and picks out the subset with the highest $\mathrm{R}^{2}$ Value. However, this method becomes infeasible for variable selection from high-dimensional data due to high computational cost (Zou and Li, 2008). Furthermore, the best subset selection suffers from several drawbacks, the most severe of which is its lack of stability as analyzed in Breiman (1996).

\subsubsection{Stepwise Multiple Regression}

Stepwise regression is one of the most widely used method of variable selection. Stepwise multiple regression algorithms operate by successive addition of significant variables (forward selection), or successive removal of significant variables (backward elimination), according to a specified criterion of variance. Whittingham (2006) examined papers published in 2004 in three leading ecological and behavioral journals (Journal of Applied Ecology, Animal Behavior, and Ecology Letters) and found that out of the 65 papers in which a multiple regression approach was used, $57 \%$ of the studies used a stepwise procedure.

However, a number of publications have drawn attention to the problems of bias arising from variable selection on the basis of statistical significance in stepwise regression (e.g. 
Anderson et al., 2000; Burnham and Anderson, 2002). Whittingham (2006) summarized that the principal drawbacks of stepwise multiple regression include bias in parameter estimation, inconsistencies among model selection algorithms, an inherent problem of multiple hypothesis testing, and an inappropriate focus or reliance on a single best model. The problem of inconsistencies, arising from the order of parameter entry (or deletion) and the number of candidate parameters (Derksen and Keselman, 1992) is particularly acute when the predictors have collinearity (Grafen and Hails, 2002).

\subsubsection{Elastic Net}

Due to the above reasons, Elastic Net, a new regularization and variable selection method proposed by Zou and Hastie (2005), was chosen in this study as the approach to select the variables and derive relationships for the spatial dynamic model. Elastic net is a hybrid of the Lasso regression (Tibshirani, 1996) and the Ridge regression (Hoerl \& Kennard 1988), both of which add a penalty term in the fitting function of ordinary linear regression, and the penalty terms are often referred to as $L_{1}$ and $L_{2}$ penalty, respectively. The Ridge regression can achieve the goal of continuous shrinkage of the parameters, but it cannot produce a parsimonious model, because it always keeps all the predictors in the model. The Lasso regression can achieve the goal of both continuous shrinkage of the parameters and automatic selection of the variables simultaneously. However, the Lasso regression tends to select only one variable from the group, among which the pairwise correlations are very high, and does not care which one is selected. If there exists high collinearity among predictors, it has been empirically observed that the prediction performance of the Lasso regression is dominated by the Ridge regression (Tibshirani, 1996). The Elastic Net used in this study can do the job of both automatically selecting the variables as the Lasso and keeping a high prediction performance when collinearity exists as the Ridge (Zou and Hastie, 2005). The regularization problem of Elastic Net is defined as minimizing the parameter $\beta_{0}$ and $\beta$ in Equation 4.1. 


$$
\frac{1}{2 N} \sum_{i=1}^{N}\left(y_{i}-\beta_{0}-x_{i}^{T} \beta\right)^{2}+\lambda \sum_{j=1}^{p}\left(\left(\frac{(1-\alpha)}{2}\right) \beta_{j}^{2}+\alpha\left|\beta_{j}\right|\right)
$$

where $N$ is the number of observations, $y_{i}$ is the value of the dependent variable at observation $i$. $x_{i}$ is a vector of the values of the independent variables at observation $i, \lambda$ is a positive regularization parameter, parameters $\beta_{0}$ and $\beta$ are the coefficients to be estimated, $\beta_{j}$ is the $j^{\text {th }}$ element in $\beta, p$ is the number of independent variables, and $\alpha$ is the parameter that determines if the regression model is more like a Lasso or a Ridge regression. As $\lambda$ increases, the number of nonzero components of $\beta$ decreases, which means the number of variables selected decreases, and vice versa. The Elastic Net is the same as a Lasso regression when $\alpha$ equals one. On the contrary, as $\alpha$ shrinks toward zero, the Elastic Net approaches a Ridge regression.

\subsubsection{Relationship Development}

From Chapter 3, all the data were spatially interpolated into the $3 \mathrm{~km}$ by $3 \mathrm{~km}$ grids. Each grid is a homogeneous unit with all the variables having a single value, and represent a spatial unit. An Elastic Net model is built with these spatial units as the sample data points, in order to derive a set of relationships for the spatial dynamic model to be developed later.

In this study, population, developed land area percentage, and utility (defined later) are stock variables in the system dynamics model, the changes of which are to be modeled. The temporal changes of these three variables between 2000 and 2010 were calculated. These changes are to be used as dependent variables, and to be predicted by independent variables at 2000 in the Elastic Net model. Each spatial unit with its stock variables' changes from 2000 to 2010 as dependent variables and its other auxiliary variables at 2000 as independent variables is one sample data point for the Elastic Net model. It should be noted that for a regression model created in this way, the assumption is that the changes in a dependent variable (e.g., population) for a given set of independent variables are the same for all spatial units on the grid space. In other words, all the spatial units have the same set of rules extracted and only different initializations. In order to eliminate the data scale impact, all the variables including their temporal changes, were standardized before input into the Elastic Net. 
In the first step, the goal was to identify the predictors of the population change. The real population change between 2000 and 2010 was input into the Elastic Net as dependent variable, and all the other social, economic, and environmental variables of 2000 (totally 35 variables) were input as independent variables. The value of $\alpha$ was set to 0.5 to consider the contributions of the $\mathrm{L}_{1}$ and the $\mathrm{L}_{2}$ penalty as half each. A cross-validated mean square error (MSE) was used to determine the value of $\lambda$, as shown in Figure 4.1, and the degree of freedom (number of predictor variables selected) under each value of $\lambda$ is shown in Figure 4.2. The cross validated MSE was calculated by dividing the whole data set into 10 groups, calibrating the Elastic Net using the first 9 groups, comparing the projected results of the $10^{\text {th }}$ group with the real data, and calculating the MSE for the $10^{\text {th }}$ group. The following equation shows the calculation of MSE.

$$
M S E=\frac{1}{N} \sum_{i=1}^{N}\left(\widehat{y}_{l}-y_{i}\right)^{2}
$$

where, $y_{i}$ is $i^{\text {th }}$ observed value, $\widehat{y}_{l}$ is the $i^{\text {th }}$ projected value, and $N$ is the number of observations.

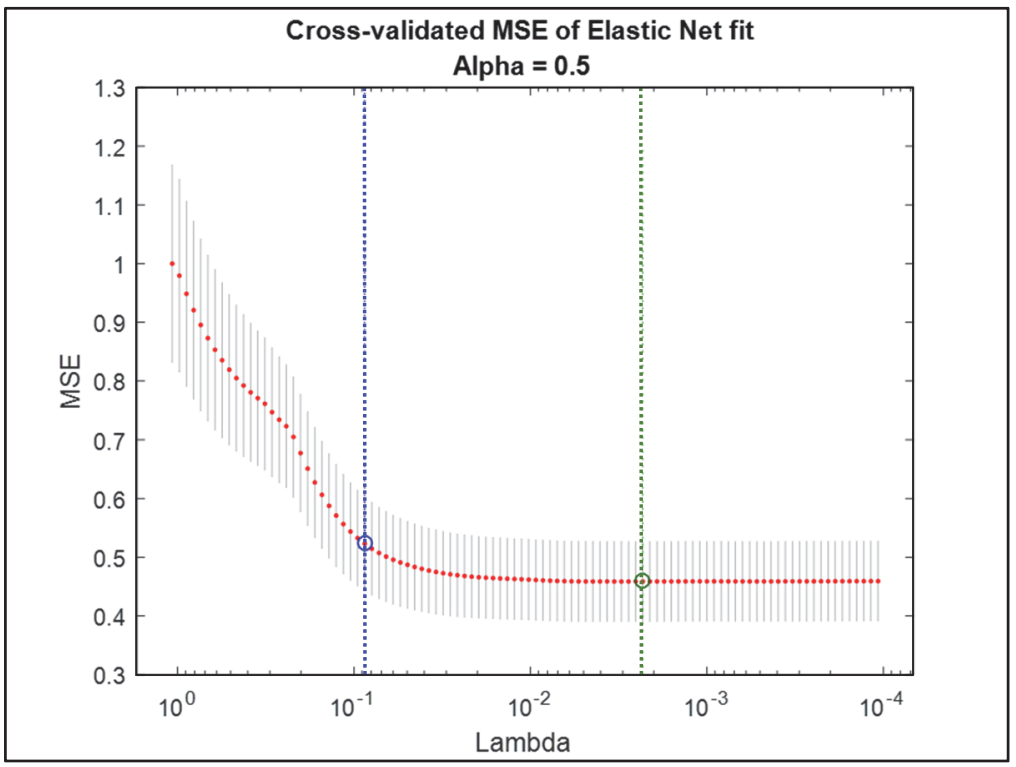

Figure 4.1 Cross Validated MSE of Elastic Net for Population Change 


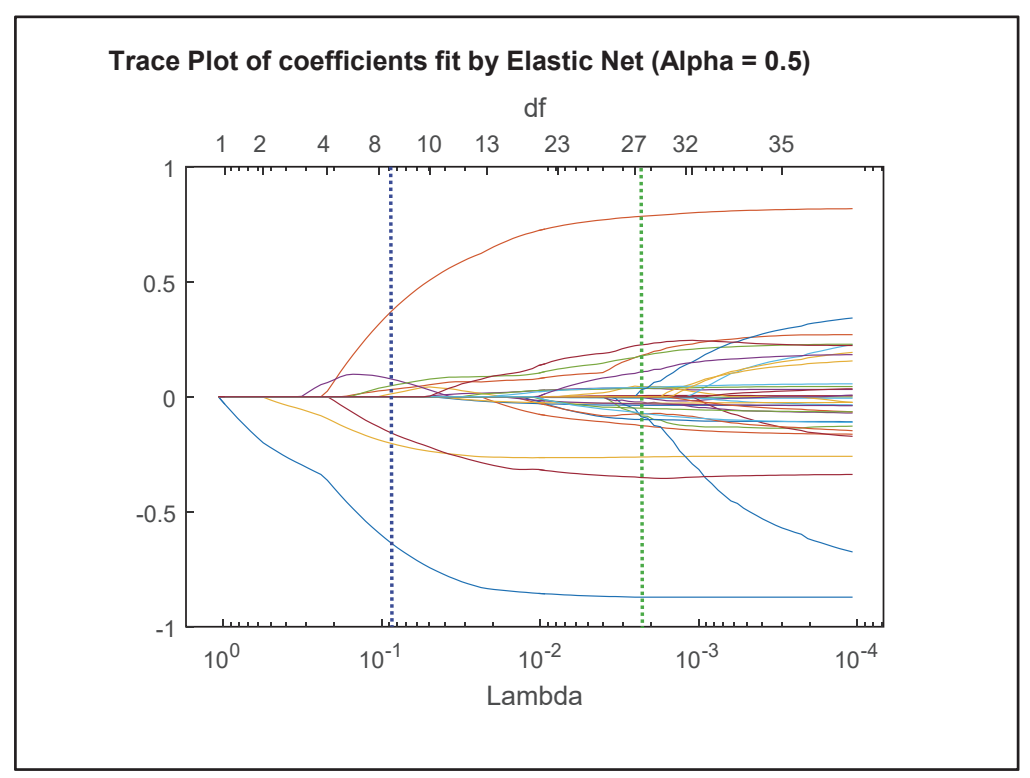

Figure 4.2 Trace Plot of Coefficients by Elastic Net for Population Change

The green dash lines in Figure 4.1 and Figure 4.2 represent the lower bound of the value of $\lambda(0.0023)$, smaller than which no predictors was trimmed. The blue dash lines in both figures represent the upper bound of the value of $\lambda(0.0870)$, greater than which the MSE will be increased significantly, which means low accuracy on prediction performance. The value of $\lambda$ was chosen as 0.0123 in this study with 17 variables included, and their standardized coefficients are plotted in Figure 4.3 and documented in Table 4.1.

Table 4.1 Standardized Coefficients of the Variables Related to Population Change Selected By Elastic Net

\begin{tabular}{|l|r|l|r|l|r|}
\hline \multicolumn{2}{|c|}{ Column 1 } & \multicolumn{2}{c|}{ Column 2 } & \multicolumn{2}{c|}{ Column 3 } \\
\hline Abbreviation & Coefficient & Abbreviation & \multicolumn{1}{c|}{ Coefficient } & Abbreviation & Coefficient \\
\hline Population & -0.8508 & Pipelines & -0.0280 & NonFuel & -0.0234 \\
\hline Developed & 0.7076 & Roads & -0.0634 & NonKitchen & -0.0109 \\
\hline Damages & -0.2646 & MedValue & 0.0745 & NonPlumb & 0.0243 \\
\hline Elevation & 0.0236 & MedRent & 0.0954 & NonTele & 0.0160 \\
\hline Water & -0.0015 & OCST35 & -0.0059 & NonVehicle & -0.3163 \\
\hline & & NonMtg & -0.0140 & Under 5 & 0.1141 \\
\hline
\end{tabular}




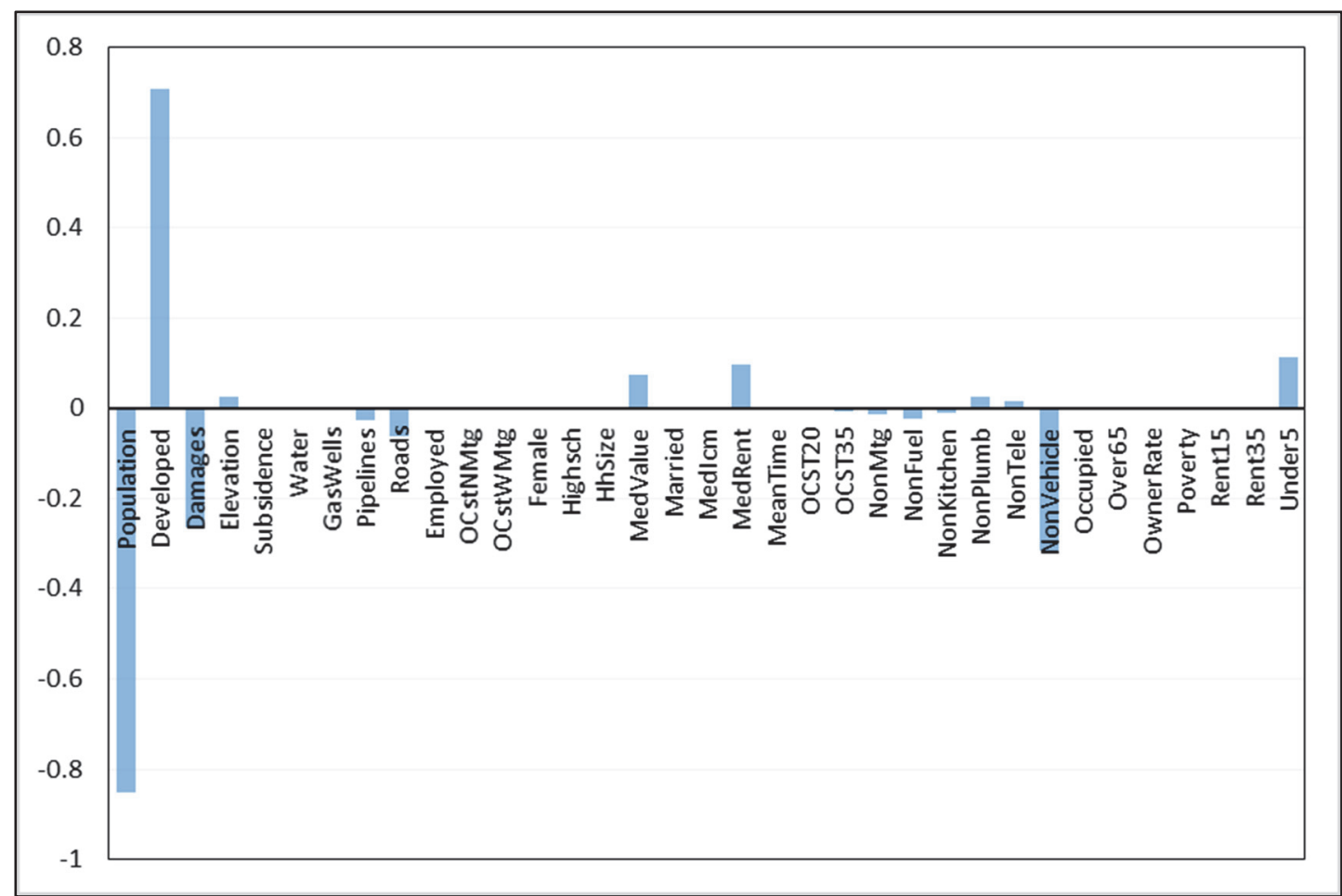

Figure 4.3 Standardized Coefficients of the Variables Related to Population Change Selected By Elastic Net

As Figure 4.3 shows, population change is highly negatively related to population, whereas it is highly positively related to developed land area percentage. The variable of property damages caused by coastal natural hazards also has a large negative effect on population change. Mean elevation slightly increases the possibility of population growth, whereas the total open water area percentage decreases it. In addition to these natural factors, there are 12 social and economic variables highly related with population change. They are combined into a single variable and termed as "utility". Utility is defined as a combination of all the socio-ecological externalities that are highly related with population change, excluding the natural and environmental externalities. Utility is defined as positively correlated with population change. In other words, positive utility will trigger population growth, whereas negative utility will trigger population loss. In this study, utility was calculated using a linear function of the 12 social and economic variables and their coefficients (column 2 and column 3 
in Table 4.1) derived from the Elastic Net model, and the calculation of Utility ${ }_{i j}$ (the utility for spatial unit $i j$ ) is shown in Equation 4.3:

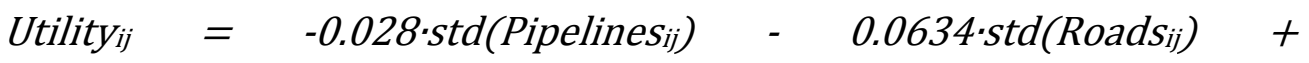

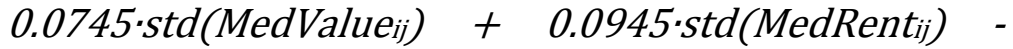

$$
\begin{aligned}
& \text { 0.0059:std(OCST35 } i j) \quad-\quad 0.0140 \cdot s t d\left(N_{i j} \text { Ontg }_{i j}\right) \quad \text { - }
\end{aligned}
$$

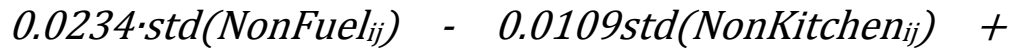

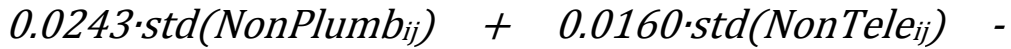

$$
\begin{aligned}
& 0.3163 \cdot s t d(\text { NonVehicle } i j)+0.1141 \cdot \text { std } \text { (Under5ij) }
\end{aligned}
$$

where the acronym of a variable (Column 2 and 3 in Table 4.1) with subscript $i j$ represents its value for spatial unit $i j$, and the function $s t d()$ means the standardization function, and the detail calculation of the standardized value of an input variable $x$ in shown in Equation 4.4:

$$
\operatorname{std}\left(x_{i j}\right)=\frac{x_{i j}-\bar{x}}{\sqrt{\frac{1}{N M} \sum_{i=1}^{N} \sum_{j=1}^{M}\left(x_{i j}-\bar{x}\right)^{2}}}
$$

where $x_{i j}$ is the value of variable $x$ for spatial unit $i j$, and $\bar{x}$ is the mean value of variable $x$ of all the spatial units.

In the second step, the goal was to select the variables that contribute the most to the change of developed land area percentage. The change of developed land area percentage was input as the dependent variable. Population, development land area percentage, the environmental variables (property damages, mean elevation, open water area, and subsidence rate), and utility (not the social and economic factors by themselves) were input as independent variables. The results are shown in Figure 4.4, and Figure 4.5. 


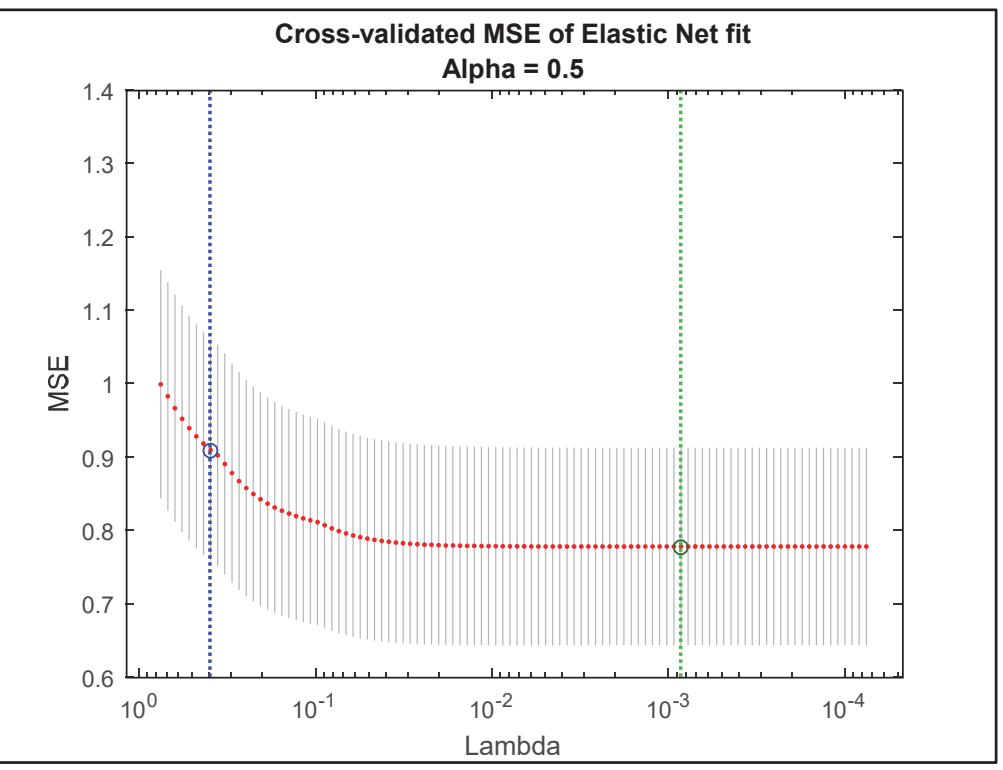

Figure 4.4 Cross Validated MSE of Elastic Net for

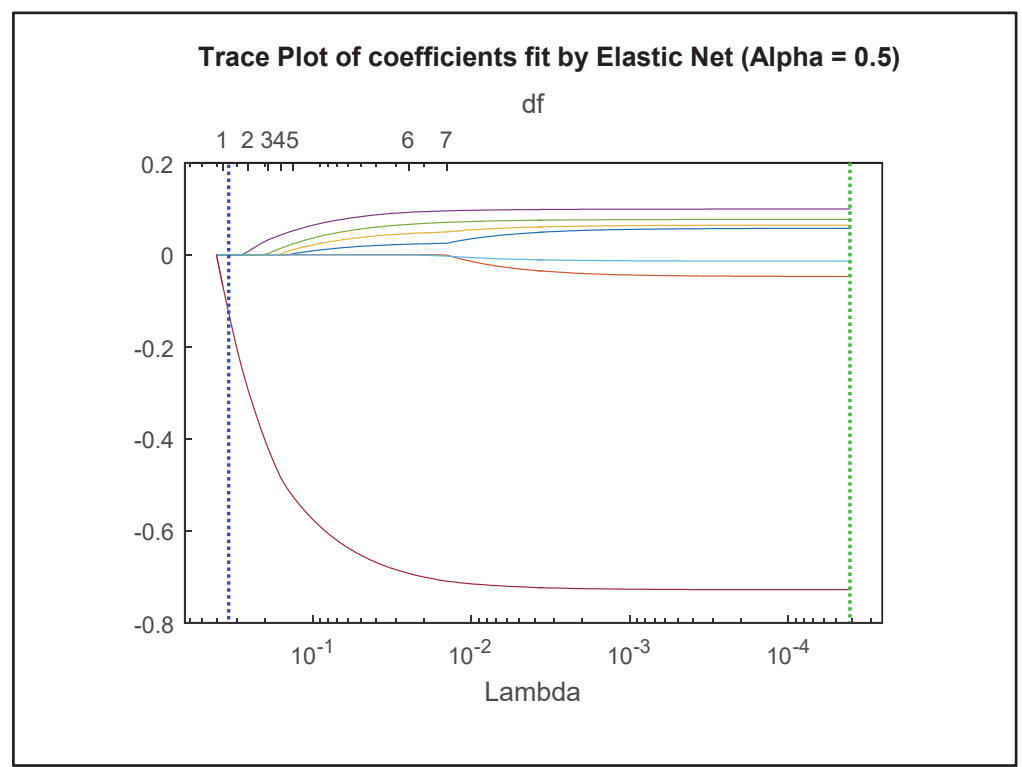

Figure 4.5 Trace Plot of Coefficients by Elastic Net for Developed Land Area Percentage Change

For the Elastic Net of the developed land area percentage, the lower bound of $\lambda$ was found to be 0.0008 , whereas the upper bound was 0.3589 , and the final value was chosen as 0.0422 with four variables selected. The standardized coefficients of the selected variables are shown in Figure 4.6 and Table 4.2. 


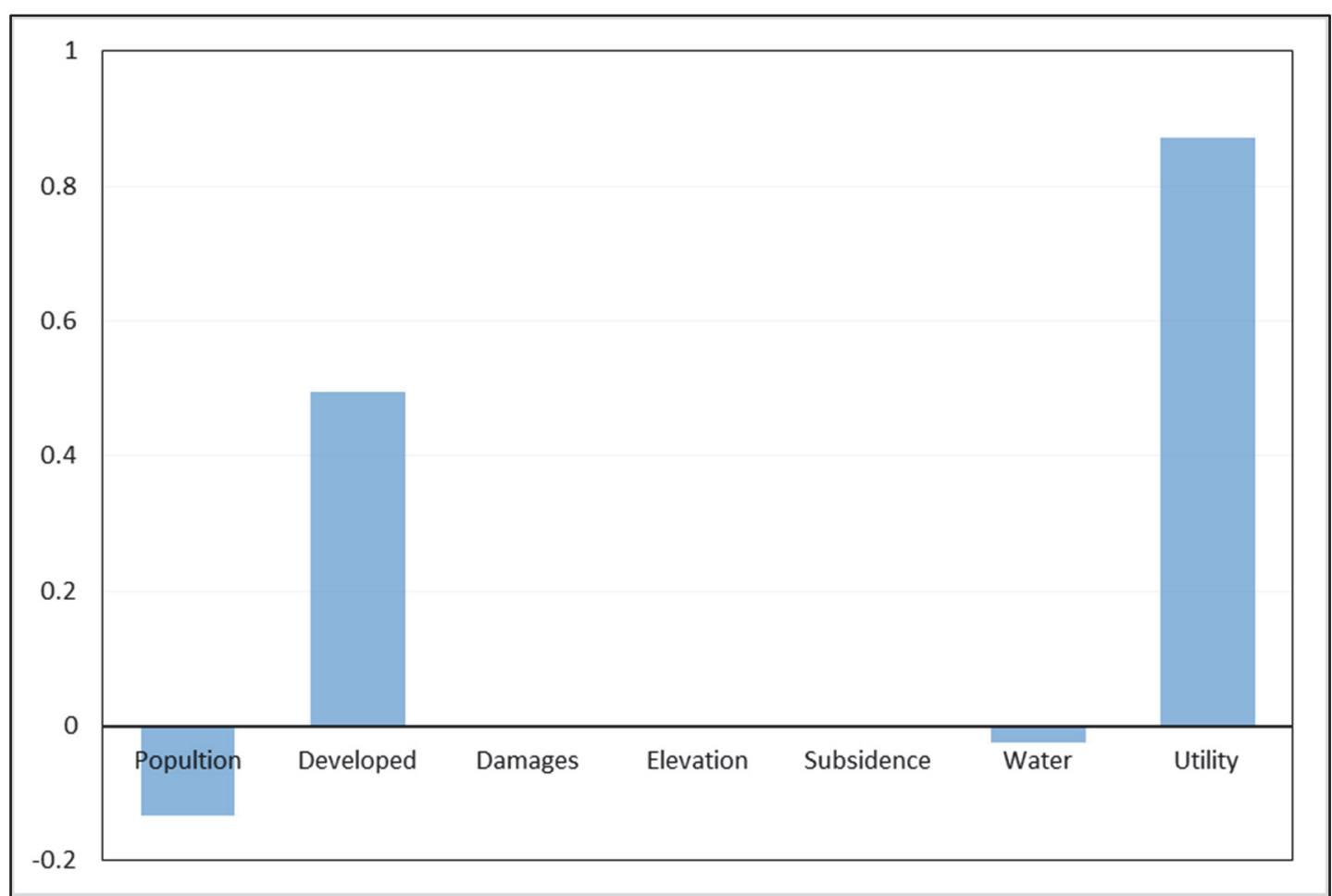

Figure 4.6 Standardized Coefficients of the Variables Related to Developed Land Area Change Selected By Elastic Net

Table 4.2 Standardized Coefficients of the Variables Related to Developed Land Area Change Selected By Elastic Net

\begin{tabular}{|l|l|l|l|l|}
\hline Abbreviation & Population & Developed & Water & Utility \\
\hline Coefficient & -0.1324 & 0.4955 & -0.0243 & 0.8706 \\
\hline
\end{tabular}

As shown in Figure 4.6, developed land area percentage and utility are the major predictors in this step, and they are positively related with the developed land area percentage change. On the other hand, high population and large open water area percentage reduce the tendency of developed land area growth.

In the third step, the goal was to find out what makes the utility change. Utility change was input as the dependent variable in this step. Population, developed land area percentage, utility value, and the environmental variables (same ones as in the second step) were input as independent variables. The results from the Elastic Net analysis in this step are shown in Figure 4.7 and Figure 4.8. 


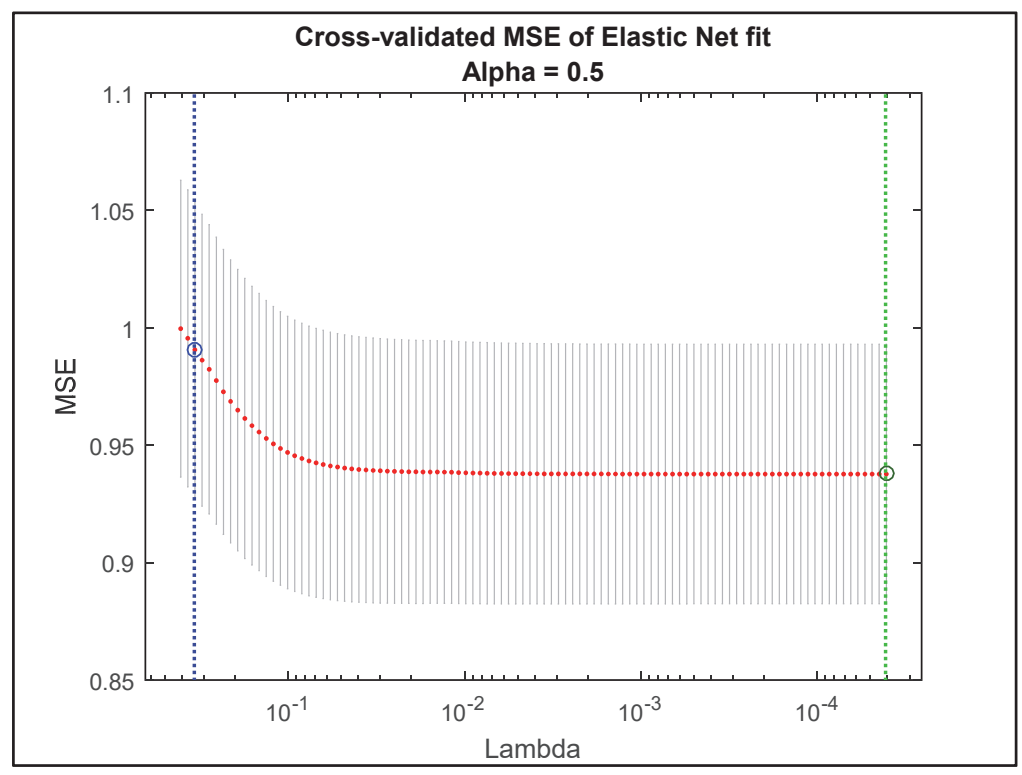

Figure 4.7 Cross Validated MSE of Elastic Net for Utility Change

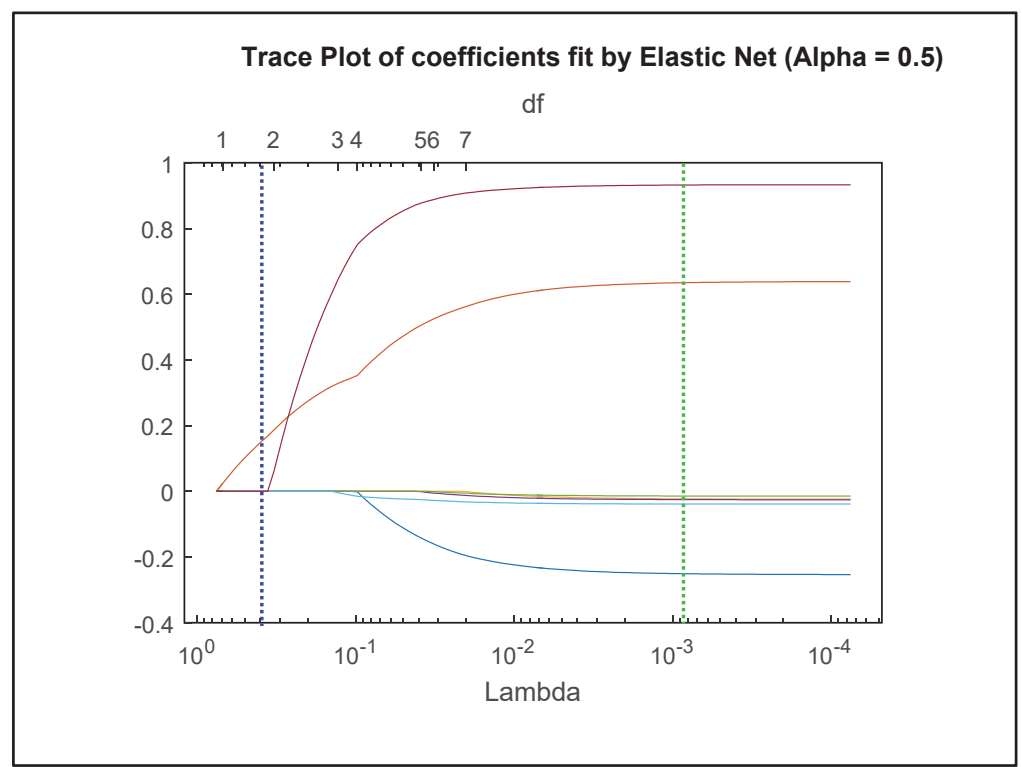

Figure 4.8 Trace Plot of Coefficients by Elastic Net for Utility Change

The lower bound was found to be $4.06 \mathrm{e}-05$, and the upper bound was 0.2117 . The final value was chosen as 0.0273 with five predictors selected. The standardized coefficients of the selected variables are shown in Figure 4.9. 


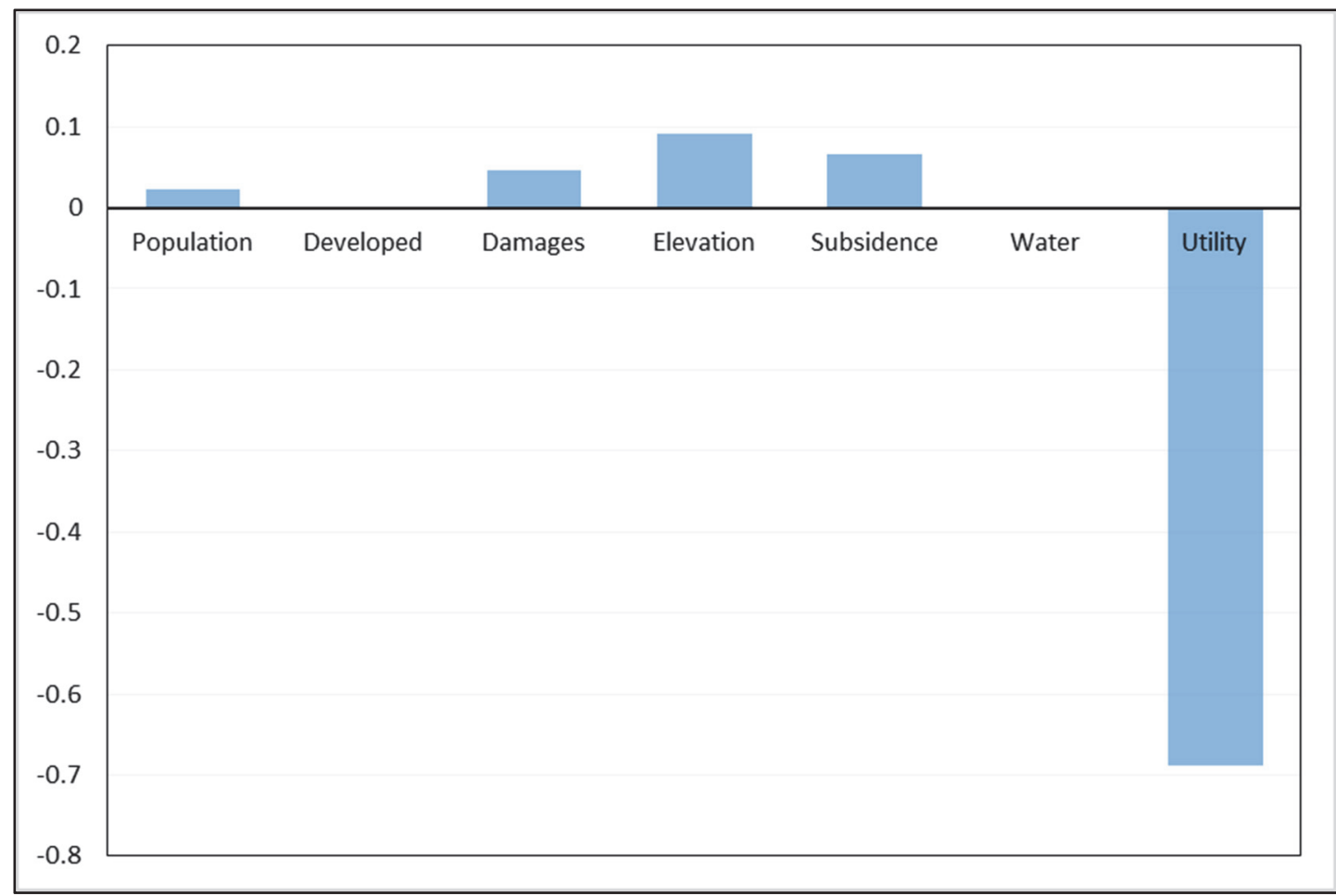

Figure 4.9 Standardized Coefficients of the Variables Related to Utility Change Selected By Elastic Net

Table 4.3 Standardized Coefficients of the Variables Related to Utility Change Selected By Elastic Net

\begin{tabular}{|l|l|l|l|l|l|}
\hline Abbreviation & Population & Damages & Elevation & Subsidence & Utility \\
\hline Coefficient & 0.0227 & 0.0453 & 0.0916 & 0.0652 & -0.6888 \\
\hline
\end{tabular}

From the results shown in Figure 4.9 and Table 4.3, population was found to have a small positive effect on increasing utility. Increases in environment disturbances (property damages and subsidence rate) seemed to increase utility, and so did mean elevation. However utility was found to have an extremely high tendency of decreasing itself. In other words, the higher the utility, the lower the utility growth.

\subsection{Coupled System Dynamics}

System dynamics modeling is an approach to discover the nonlinear behavior of complex systems over time using stock variables and flows (the changes of the stock variables). It is "a computer-aided approach to theory building, policy analysis, and strategic decision support emerging from an endogenous point of view" (Richardson, 2008). The field was 
developed initially from the work of Jay W. Forrester (1961) in industry dynamics. After decades it has been extended to many dynamic problems arising in complex social, managerial, economic, and ecological systems, and literally any dynamic systems characterized by interdependence, mutual interaction, information feedback, and circular causality. System dynamics modeling uses stocks and flows to quantitatively analyze the feedback loops. Stocks are the variables that accumulate or deplete over time, whereas flows are the changing rates of these stocks. The establishment of a system dynamics model includes a graphic diagram corresponding to the structure of feedback loops and a set of calibrated parameters. Mathematically, the basic structure of a formal system dynamics model is a set of coupled, nonlinear, first-order differential (or integral) equations.

This section describe the procedure of building the system dynamics as shown in Figure 4.10 .

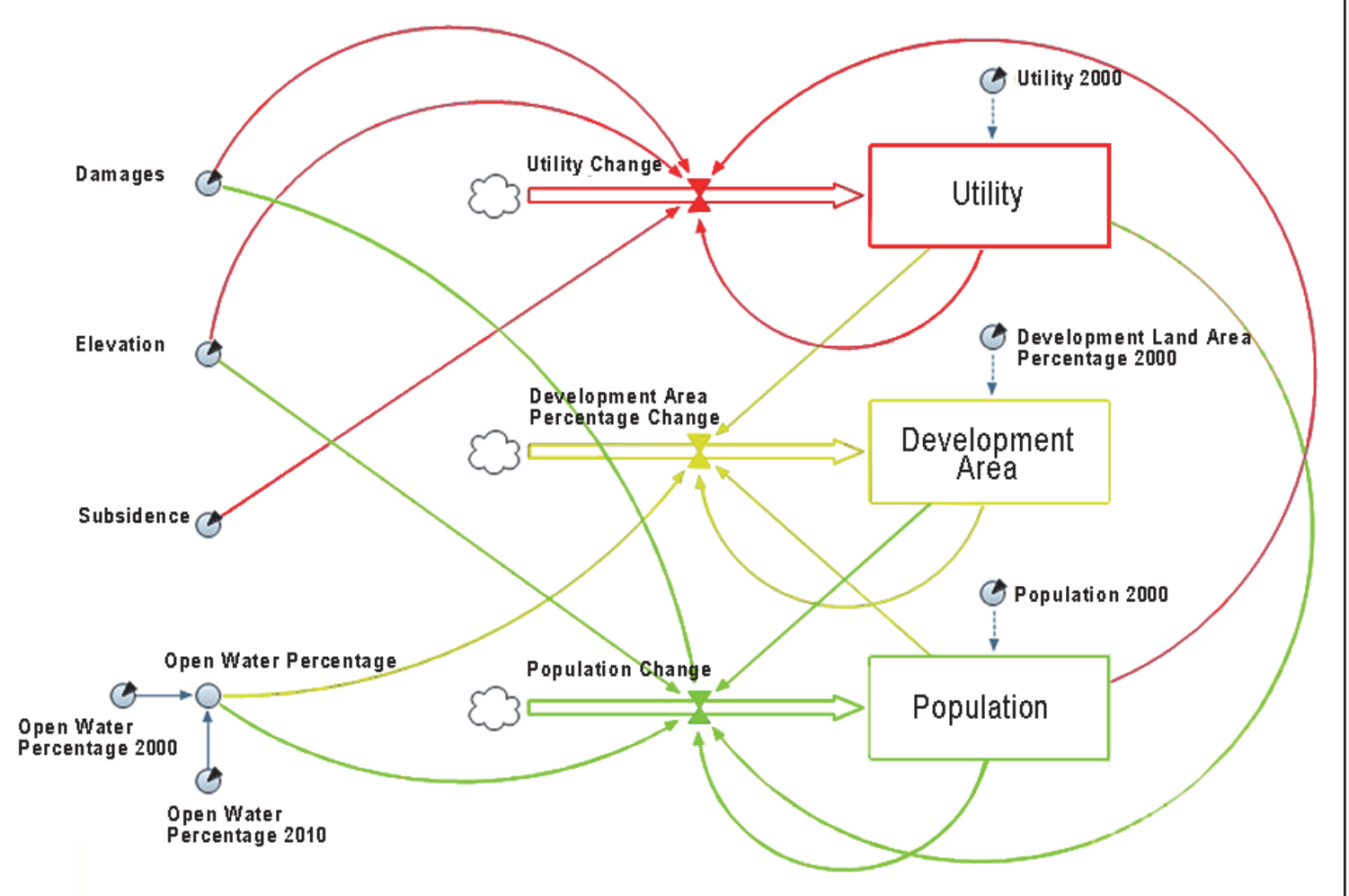

Figure 4.10 The Coupled System Dynamics Diagram 
In this study, difference equations were used for updating the values of the variables over time. All the spatial units use the same set of difference equations for their system dynamics but with their own initial values. The description of all the parameters in the equations are in Table 4.4. The description of all the variables in the equations denoted by their acronyms are in Table 4.5 .

Table 4.4 Symbols, Units and Definitions for the Coefficients of the Equations in the Spatial Dynamic Model

\begin{tabular}{|c|c|c|c|c|}
\hline Symbol & Unit & Definition & Equation & Calibration \\
\hline $\mathrm{a}_{1}$ & 1/dollar & Coefficient of utility change dependency on damages & $4.5 ; 5.1$ & Elastic Net \\
\hline $\mathrm{b}_{1}$ & $1 / \mathrm{m}$ & $\begin{array}{l}\text { Coefficient of utility change dependency on } \\
\text { elevation }\end{array}$ & $4.5 ; 5.1$ & Elastic Net \\
\hline $\mathrm{c}_{1}$ & year $/ \mathrm{m}$ & $\begin{array}{l}\text { Coefficient of utility change dependency on } \\
\text { subsidence }\end{array}$ & $4.5 ; 5.1$ & Elastic Net \\
\hline $\mathrm{d}_{1}$ & - & Coefficient of utility change dependency on utility & $4.5 ; 5.1$ & Elastic Net \\
\hline $\mathrm{f}_{1}$ & $1 /$ number & $\begin{array}{l}\text { Coefficient of utility change dependency on } \\
\text { population }\end{array}$ & $4.5 ; 5.1$ & Elastic Net \\
\hline$d_{2}$ & - & $\begin{array}{l}\text { Coefficient of developed change dependency on } \\
\text { utility }\end{array}$ & $4.6 ; 5.2$ & Elastic Net \\
\hline $\mathrm{e}_{2}$ & - & $\begin{array}{l}\text { Coefficient of developed change dependency on } \\
\text { developed }\end{array}$ & $4.6 ; 5.2$ & Elastic Net \\
\hline$f_{2}$ & $1 /$ number & $\begin{array}{l}\text { Coefficient of developed change dependency on } \\
\text { population }\end{array}$ & $4.6 ; 5.2$ & Elastic Net \\
\hline $\mathrm{g}_{2}$ & - & $\begin{array}{l}\text { Coefficient of developed change dependency on } \\
\text { water }\end{array}$ & $4.6 ; 5.2$ & Elastic Net \\
\hline$a_{3}$ & number/dollar & $\begin{array}{l}\text { Coefficient of population change dependency on } \\
\text { damages }\end{array}$ & $4.7 ; 5.3$ & Elastic Net \\
\hline $\mathrm{b}_{3}$ & number/meter & $\begin{array}{l}\text { Coefficient of population change dependency on } \\
\text { elevation }\end{array}$ & $4.7 ; 5.3$ & Elastic Net \\
\hline$d_{3}$ & number & $\begin{array}{l}\text { Coefficient of population change dependency on } \\
\text { utility }\end{array}$ & $4.7 ; 5.3$ & Elastic Net \\
\hline$e_{3}$ & number & $\begin{array}{l}\text { Coefficient of population change dependency on } \\
\text { developed }\end{array}$ & $4.7 ; 5.3$ & Elastic Net \\
\hline$f_{3}$ & - & $\begin{array}{l}\text { Coefficient of population change dependency on } \\
\text { population }\end{array}$ & $4.7 ; 5.3$ & Elastic Net \\
\hline $\mathrm{g}_{3}$ & number & $\begin{array}{l}\text { Coefficient of population change dependency on } \\
\text { water }\end{array}$ & $4.7 ; 5.3$ & Elastic Net \\
\hline
\end{tabular}


Table 4.5 Acronyms, Types, Units, Definitions, and Updating Rules of the Variables in the Spatial Dynamic Model

\begin{tabular}{|c|c|c|c|c|}
\hline Acronym & Type & Unit & Definition & $\begin{array}{l}\text { Updating } \\
\text { Rule }\end{array}$ \\
\hline Population & State & number & Total Population of a Spatial Unit & $\begin{array}{l}\text { Equation } \\
4.5 \text { or } 5.1\end{array}$ \\
\hline Developed & State & - & Percent of Developed Land Use Area & $\begin{array}{l}\text { Equation } \\
4.6 \text { or } 5.2\end{array}$ \\
\hline Utility & State & - & $\begin{array}{l}\text { A Combination of all the Socio-Ecological Externalities } \\
\text { that Highly Related with Population Change (Equation } \\
4.5)\end{array}$ & $\begin{array}{l}\text { Equation } \\
4.7 \text { or } 5.3\end{array}$ \\
\hline Damages & Auxiliary & $\begin{array}{l}\text { dollar/ } \\
\text { year }\end{array}$ & $\begin{array}{l}\text { Average Property Damages Caused by Coastal Natural } \\
\text { Hazards per Year }\end{array}$ & Constant \\
\hline Elevation & Auxiliary & $\mathrm{m}$ & Mean Elevation & Constant \\
\hline Subsidence & Auxiliary & m/year & $\begin{array}{l}\text { Average Subsidence Rate per Year Measured by Bench } \\
\text { Mark }\end{array}$ & Constant \\
\hline Water & Auxiliary & - & Percent of Open Water Land Use Area & $\begin{array}{l}\text { Equation } \\
4.8\end{array}$ \\
\hline Pipelines & Auxiliary & $1 / \mathrm{m}^{2}$ & Kernel Density of Oil and Gas Pipelines & Constant \\
\hline Roads & Auxiliary & $1 / \mathrm{m}^{2}$ & Kernel Density of Traffic Roads & Constant \\
\hline MedValue & Auxiliary & dollar & Median Value of Specified Owner Occupied Units & Constant \\
\hline MedRent & Auxiliary & dollar & Median Gross Rent of Specified Renter Occupied Units & Constant \\
\hline OCST35 & Auxiliary & - & $\begin{array}{l}\text { Percent of Households with Owner Cost more than } 35 \\
\text { Percent of their Household Income }\end{array}$ & Constant \\
\hline NonMtg & Auxiliary & - & $\begin{array}{l}\text { Percent of Specified Owner Occupied Units without } \\
\text { Mortgage }\end{array}$ & Constant \\
\hline NonFuel & Auxiliary & - & $\begin{array}{l}\text { Percent of Occupied Housing Units with No House } \\
\text { Heating Fuel Used }\end{array}$ & Constant \\
\hline NonKitchen & Auxiliary & - & $\begin{array}{l}\text { Percent of Occupied Housing Units Lacking Complete } \\
\text { Plumbing Facilities }\end{array}$ & Constant \\
\hline NonPlumb & Auxiliary & - & $\begin{array}{l}\text { Percent of Occupied Housing Units - Lacking complete } \\
\text { Kitchen Facilities }\end{array}$ & Constant \\
\hline NonTele & Auxiliary & - & $\begin{array}{l}\text { Percent of Occupied Housing Units with No Telephone } \\
\text { Service }\end{array}$ & Constant \\
\hline NonVehicle & Auxiliary & - & $\begin{array}{l}\text { Percent of Occupied Housing Units with None Vehicles } \\
\text { Available }\end{array}$ & Constant \\
\hline Under5 & Auxiliary & - & Percent of Total Population under 5 Years Old & Constant \\
\hline
\end{tabular}

The difference equations are derived by the Elastic Net regression equations described in the last section (Section 4.1), with their coefficients divided by 10, since the Elastic Net was calibrated to project the changes of the stock variables for ten years. Thus each time step of running the difference equations in the model represents one year. The deference equations for updating the three stock variables are shown in Equation 4.5, 4.6, and 4.7. 


$$
\begin{aligned}
\text { Population }_{i j}(t+1)= & \text { Population }_{i j}(t)+f_{3} \cdot \text { Population }_{i j}(t)+e_{3} \cdot \text { Developed }_{i j} \\
& (t)+a_{3} \cdot \text { Damages }_{i j}+b_{3} \cdot \text { Elevation }_{i j}+g_{3} \cdot \text { Water }_{i j}(t)+ \\
& d_{3} \cdot \text { Utilityij }_{i j}(t)
\end{aligned}
$$

$$
\begin{aligned}
& \text { Developed }_{i j}(t+1)=\text { Developed }_{i j}(t)+f_{2} \cdot \text { Population }_{i j}(t)+e_{2} \cdot \text { Developed }_{i j} \\
& (t)+g_{2} \cdot \text { Water }_{i j}(t)+d_{2} \cdot \text { Utilityij }_{i j}(t) \\
& \text { Utilityij }_{i j}(t+1)=\text { Utilityij }_{i j}(t)+f_{1} \cdot \text { Population }_{i j}(t)+a_{1} \cdot \text { Damagesij }+b_{1} \cdot \\
& \text { Elevation }_{i j}+c_{1} \cdot \text { Subsidence }_{i j}+d_{1} \cdot \text { Utility }_{i j}(t)
\end{aligned}
$$

where subscript $i j$ refers to the spatial unit $(i, j)$ in row $i$ and column $j$, and $t$ denotes a certain time step.

Equation 5.1, 5.2, and 5.3 mentioned in Table 4.4 and 4.5 will be introduced in Chapter 5. The environmental variables of Damages, Subsidence and Elevation are not updated over time during the simulation. They were not modeled in this study due to the temporal data availability. For the environmental variable of Water, although it was not modeled, it is updated with a constant rate calculated by the historical data between 2000 and 2010. The deference equation is shown in Equation 4.7.

$$
\text { Waterij }_{i+1}\left(t+\text { Waterij }_{i j}(t)+\left(\text { Water }_{i j}(2010)-\text { Water }_{i j}(2000)\right) / 10\right.
$$

where, subscript $i j$ refers to the spatial unit in row $i$ and column $j, t$ denotes a certain time step, and 2000 and 2010 denote the time step at the year 2000 and the year 2010.

The system dynamics model with these coupled difference equations was run on all of the spatial units for ten time steps, and the simulated results were compared with the real data of 2010 (Figure 4.11 and Figure 4.12). The initialization of the system dynamics used the real data of 2000. The initialization of utility used Equation 4.3. Three constraints were added to the simulation: (1) if the population of a spatial unit is below zero, then it cannot receive negative flows to its population in the next time step, (2) if the developed area percentage of a spatial unit is over $100 \%$, then it cannot receive positive flows to its developed area percentage 
in the next time step. (3) Since from the historical data, by which the model was calibrated, there was no developed area decreases, it was assumed that the developed area percentage cannot have negative flows. If a negative flow is projected by the difference functions, then it is set to zero. The prediction accuracies of using the Elastic Net regression function directly are also shown in Figure 4.13 and Figure 4.14 for comparison.

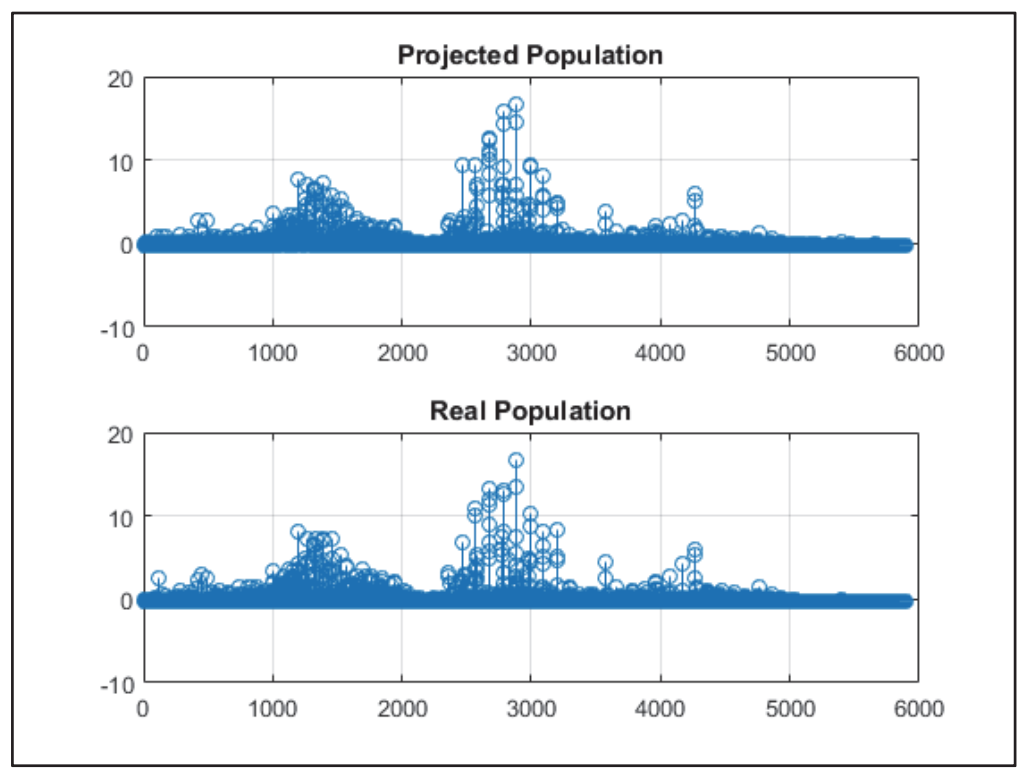

Figure 4.11 Comparison between the Predicted and the Real Population (Standardized) in 2010 by Spatial Units (System Dynamics)

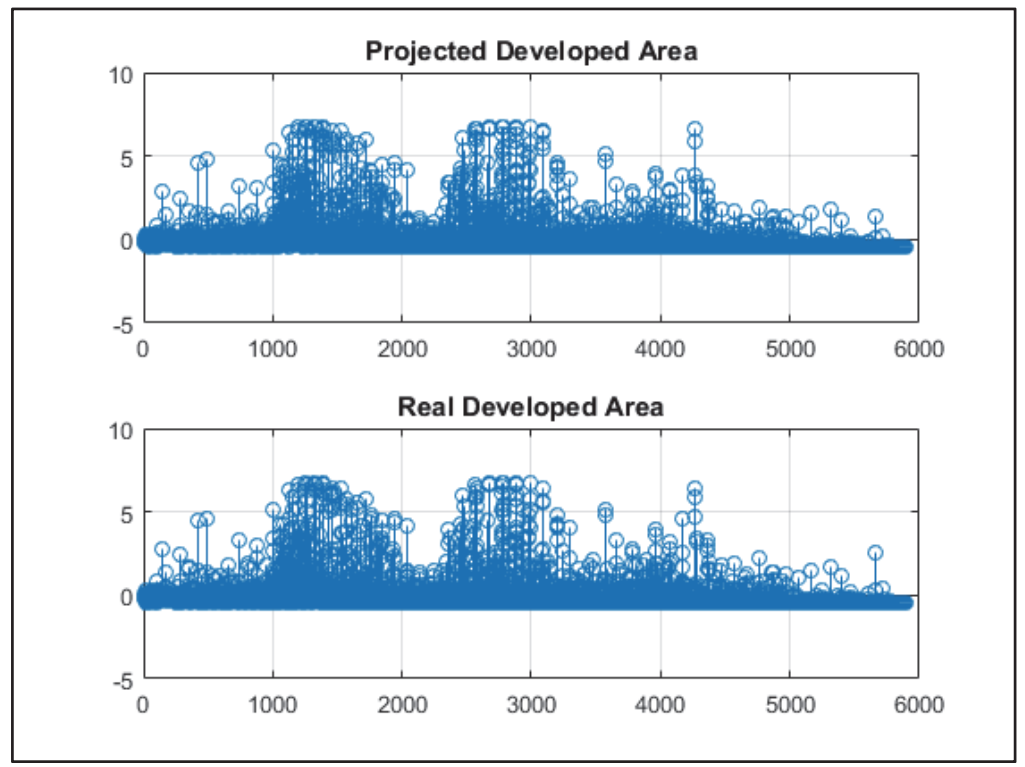

Figure 4.12 Comparison between the Predicted and the Real Developed Land Area Percentage (Standardized) in 2010 by Spatial Units (System Dynamics) 

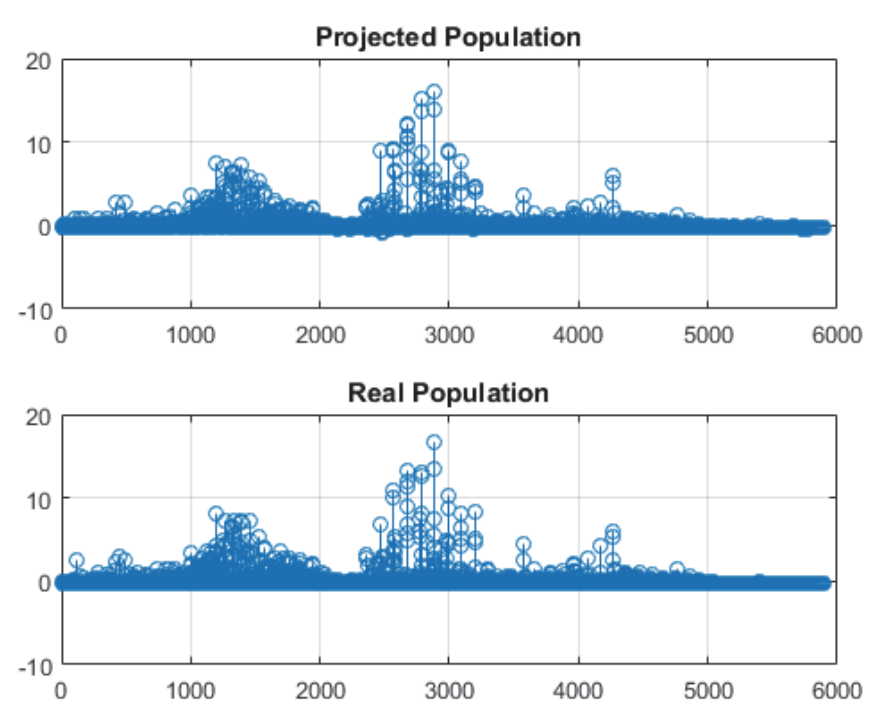

Figure 4.13 Comparison between the Predicted and the Real Population (Standardized) in 2010 by Spatial Units (Elastic Net)

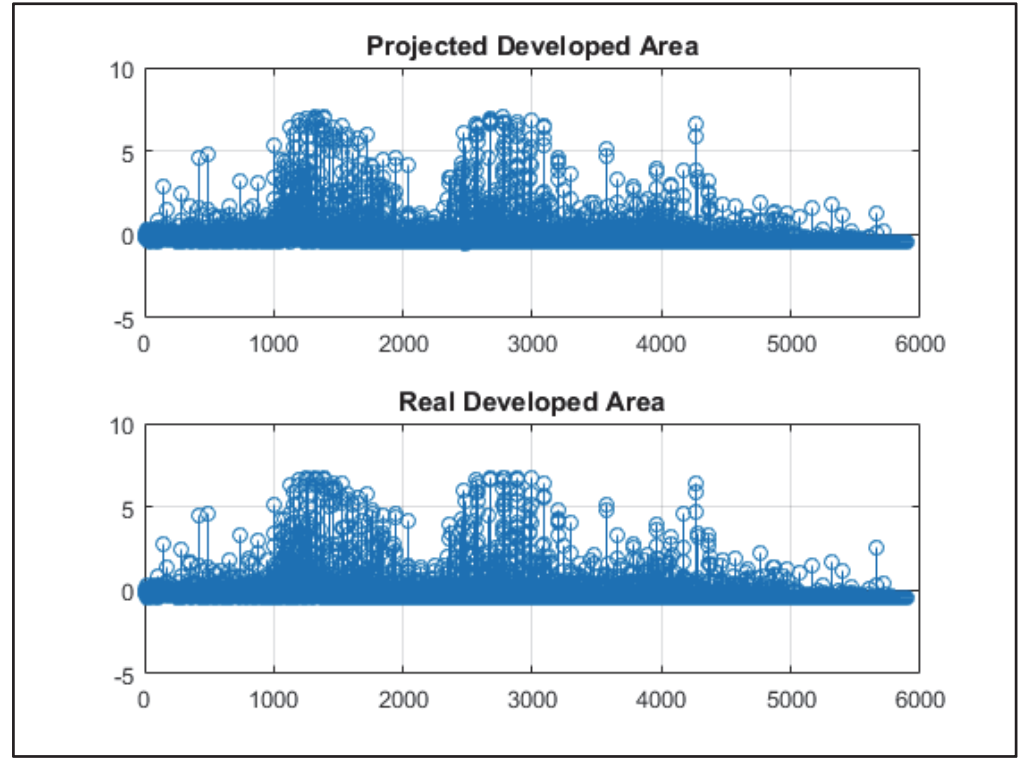

Figure 4.14 Comparison between the Predicted and the Real Developed Land Area Percentage (Standardized) in 2010 by Spatial Units (Elastic Net)

The mean squared error (MSE) measurement (Equation 4.2) was used to indicate the prediction accuracy. Calculated from the data in Figure 4.11, Figure 4.12, Figure 4.13 and Figure 4.14, the MSEs for using Elastic Net directly to predict population and development land area percentage was 0.1350 and 0.0622 respectively. The MSEs for using the system dynamics model built by the functions derived by the Elastic Net was 0.1369 and 0.0615 for 
population and development land area percentage, respectively. By comparing the MSEs, it was found that the prediction accuracies of the system dynamics model determined by the Elastic Net was maintained. In the meantime, the linear prediction functions from the Elastic Net were transformed to the non-linearly coupled feedback loops in the system dynamics model. Although the Elastic Net Model by itself has the ability to predict, but if directly using the linear prediction functions from it to predict, the increasing and decreasing trends of the variables to be predicted will never change, since the Elastic Net functions are never updated and always use the initial values of the predictors to guide the future change. By using the system dynamics, the prediction rules are still kept and the perdition accuracy is maintained. Most importantly, since the prediction functions are updated by the instant values of the predictor variables at each time step, the existence of the emergence resulting from the coupled dynamics can be tested in the future simulation. 


\subsection{References}

Anderson, D.R., Burnham, K.P., Thompson, W.L. (2000). Null hypothesis testing: problems, prevalence, and an alternative. Journal of Wildlife Management 64: 912-923.

Breiman, L. (1996). Heuristics of instability and stabilization in model selection. The Annals of Statistics. 24: 2350-2383.

Burnham, K.P., Anderson, D.R. (2002). Model selection and multimodel inference: a practice information theoretic approach. Springer Verlag, New York.

Derksen, S., Keselman, H.J. (1992). Backward, forward and stepwise automated subset selection algorithms: frequency of obtaining authentic and noise variables. British Journal of Mathematical and Statistical Psychology 45: 265-282.

Fan, J., Li, R. (2006). Statistical challenges with high dimensionality: Feature selection in knowledge discovery. In Proceedings of the Madrid International Congress of Mathematicians 3: 595-622. EMS, Zürich.

Forrester, J.W. (1961). Industrial dynamics. Cambridge, MA: The MIT Press. Reprinted by Pegasus Communications, Cambridge, MA.

Grafen, A., Hails, R. (2002). Modern statistics for the life sciences. Oxford University Press, Oxford.

Hoerl, A., Kennard, R. (1988) Ridge regression. In Encyclopedia of Statistical Sciences. New York: Wiley 8: 129-136.

Richardson, G. P. (2008). The Core of System Dynamics. Encyclopedia of Complexity and Systems Science.

Tibshirani, R. (1996). Regression shrinkage and selection via the lasso. J. R. Statist. Soc. B 58: 267-288.

Whittingham, J., Stephens, A., Bradbury, B. And Freckleton, P. (2006). Why do we still use stepwise modelling in ecology and behavior? Journal of Animal Ecology 75(5).

Zou, H., Hastie, T. (2005). Regularization and variable selection via the elastic net. Journal of the Royal Statistical Society, Series B, 67(2): 301-320.

Zou, H., Li, R. (2008). One-step sparse estimates in non-concave penalized likelihood models The Annals of Statistics 36(4): 1509-1533. 


\section{CHAPTER 5 : SPATIAL DANAMIC MODEL AND GENETIC ALGORITHM}

This chapter describes the development of the spatial dynamic model to include the neighborhood effects. The neighborhood effects rules were derived from the rules extracted from Chapter 4, and reflect the autonomy, the reactivity, and the interaction ability. The autonomy of the spatial units were reflected by the difference equations' dependencies on the spatial units' attributes. The reactivity of the spatial units were reflected by the difference equations' dependencies on the environmental variables. The major difference between the spatial dynamic model of Chapter 5 and Chapter 4 is that the spatial units have interactions with each other in Chapter 5, and new neighborhood effects terms were added to their difference equations. Genetic Algorithms were used to calibrate the coefficients in these terms. Monte Carlo simulation with simple random sampling method was conducted for the uncertainty analysis.

\subsection{Spatial Dynamic Modeling}

In this model, the neighborhood is defined as the Moore neighborhood (the eight contiguous neighbors). Three neighborhood effects terms were added to Equation 4.5, 4.6, and 4.7, as shown in Equation 5.1, 5.2, and 5.3:

$$
\begin{aligned}
& \text { Population }_{i j}(t+1)=\text { Population }_{i j}(t)+\left(1-P_{1}\right) *\left(f_{3} \cdot \text { Population }_{i j}(t)+\right. \\
& e_{3} \cdot \text { Developed }_{i j}(t)+a_{3} \cdot \text { Damages }_{i j}+b_{3} \cdot \text { Elevation }_{i j}+ \\
& \left.g_{3} \cdot \text { Waterij }_{i j}(t)+d_{3} \cdot \text { Utilityij }_{i j}(t)\right)+P_{1} \text { * } \\
& \left(\frac{1}{8} \sum_{k=1}^{8} \text { Population }_{N_{k}}(t+1)-\text { Population }_{N_{k}}(t)\right) \\
& \text { Developed }_{i j}(t+1)=\text { Developed }_{i j}(t)+\left(1-P_{2}\right) *\left(f_{2} \cdot \text { Population }_{i j}(t)+\right. \\
& \left.e_{2} \cdot \operatorname{Developed}_{i j}(t)+g_{2} \cdot \text { Water }_{i j}(t)+d_{2} \cdot \operatorname{Utility}_{i j}(t)\right) \\
& +P_{2} *\left(\frac{1}{8} \sum_{k=1}^{8} \text { Developed }_{N_{k}}(t+1)-\right. \\
& \text { Developed } \left._{N_{k}}(t)\right)
\end{aligned}
$$




$$
\begin{aligned}
\text { Utilityij }_{i j}(t+1)= & \text { Utilityij }_{i j}(t)+\left(1-P_{3}\right) *\left(f_{1} \cdot \text { Population }_{i j}(t)+a_{1} \cdot \text { Damages }_{i j}\right. \\
& \left.+b_{1} \cdot \text { Elevation }_{i j}+c_{1} \cdot \text { Subsidence }_{i j}+d_{1} \cdot \text { Utilityij }_{i j}(t)\right)+P_{3} \\
& *\left(\frac{1}{8} \sum_{k=1}^{8} \text { Utility }_{N_{k}}(t+1)-\text { Utility }_{N_{k}}(t)\right)
\end{aligned}
$$

where subscript $i j$ refers to a spatial unit $(i, j)$ in row $i$ and column $j, t$ denotes a certain time step, and the description of all the parameters except for the ones in the neighborhood terms can be found in Table 4.4. The description of all the variables in the equations denoted by their acronyms can be found in Table 4.5. $P_{1}, P_{2}$, and $P_{3}$ are parameters that determine neighborhood effects weights, $P_{1}$ is the direct neighborhood impact parameter for population change, $P_{2}$ is the direct neighborhood impact parameter for developed area percentage change, and $P_{3}$ is the direct neighborhood impact parameter for utility change. It should be noted that $P_{1}, P_{2}$, and $P_{3}$ also have indirect neighborhood impacts on other stock variables through the system dynamics. $N_{k}$ is the $k^{\text {th }}$ element of the Moore neighborhood set $(N)$ of spatial unit $(i, j)$, and $N=\{(i-1, j-1)$, $(i-1, j),(i-1, j+1),(i, j-1),(i, j+1),(i+1, j-1),(i+1, j),(i+1, j+1)\}$. These equations reflecting the interactions among the spatial units are shown in Figure 5.1 (a revision of the diagram of Figure 4.10). 


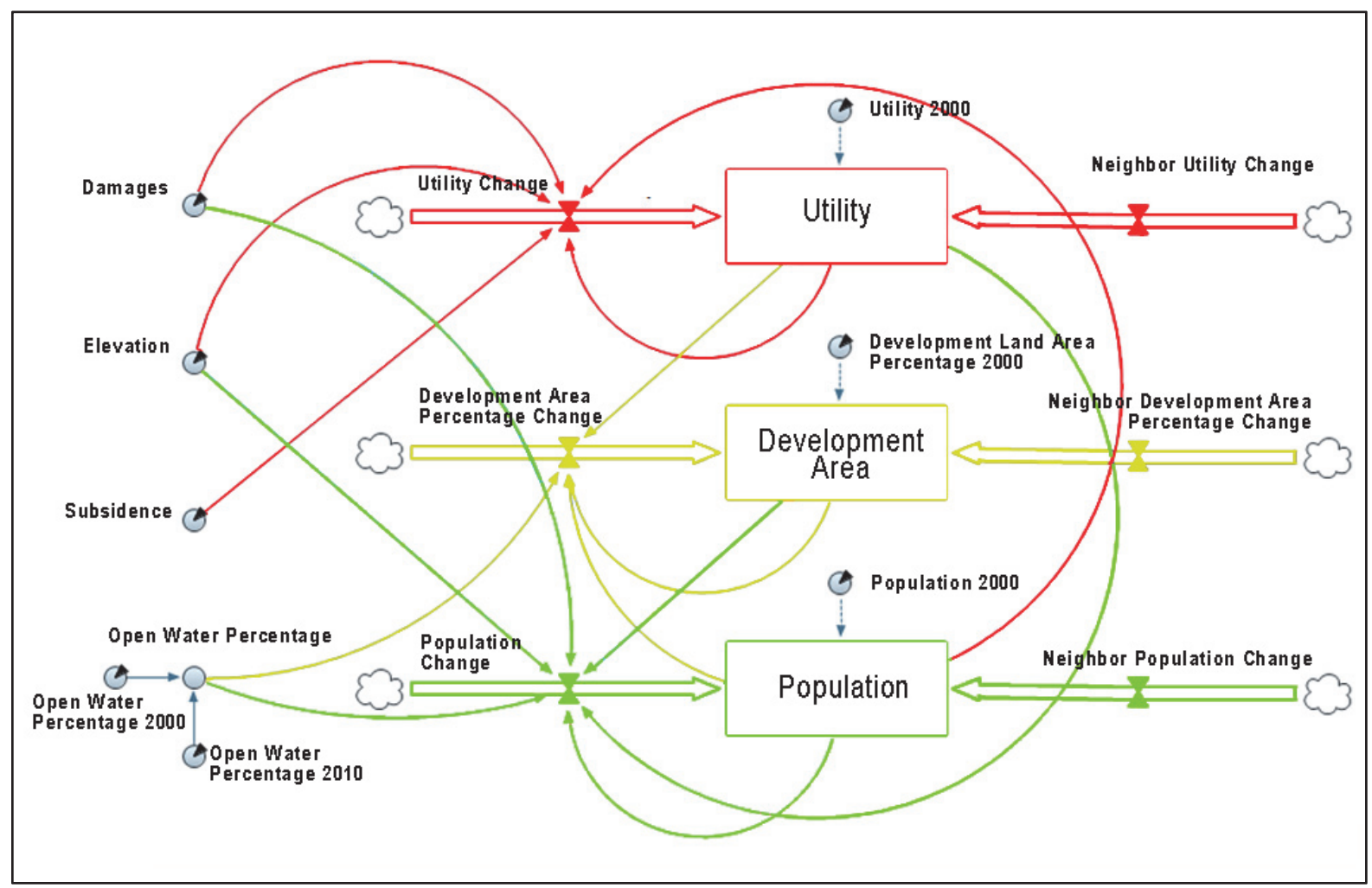

Figure 5.1 The Spatial Coupled Dynamics Diagram

From Figure 5.1, the changes of the stock variables are determined by both the "flows" of itself and the "flows" of its neighbor spatial units. The eight Moore neighbors of a spatial unit are assumed to have equal neighborhood impacts on it.

Assuming that the neighborhood impacts contribute 50\% to the changes of all the three stock variables $\left(P_{1}=0.5, P_{2}=0.5\right.$, and $\left.P_{3}=0.5\right)$, a simulation was run from 2000 to 2010 using all the spatial units. Under this assumption, the changes of population, utility, and developed area percentage in a spatial unit are the means of the flows calculated by the system dynamics and the average flows calculated by its Moore neighbors' system dynamics. The model constraints and initializations mentioned in Chapter 4 (Section 4.2) still stand in this simulation. The simulated results are shown in Figure 5.2 and Figure 5.3. 

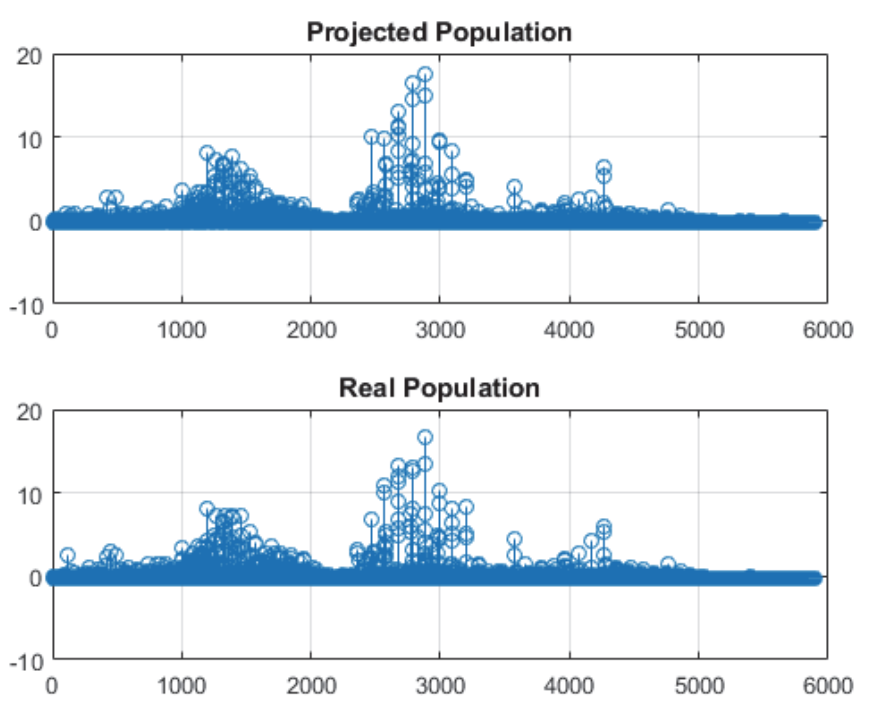

Figure 5.2 Comparison between the Predicted and the Real Population (Standardized) in 2010 by Spatial Units (with Neighborhood Effects)
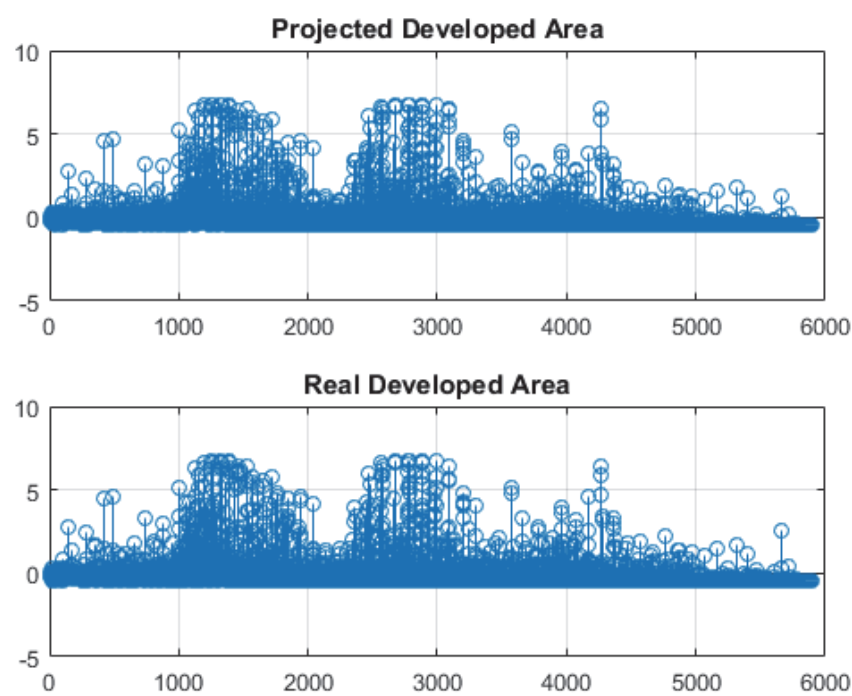

Figure 5.3 Comparison between the Predicted and the Real Developed Land Area Percentage (Standardized) in 2010 by Spatial Units (with Neighborhood Effects)

The MSE for the simulated population was 0.1417 , and the MSE for the simulated development land area percentage was 0.0610. The MSE for the population simulated with neighborhood effects is higher than without neighborhood effects (Chapter 4), whereas the MSE for the simulated development land area percentage is lower, and the sum of them is a little bit higher. Thus it is noted that adding the interactions among the spatial units does not 
make the prediction more accurate. Since the simulation was tested under the assumption that the percentage of the impacts from neighborhood on the changes of the three stock variables are all $50 \%$, whether the prediction accuracies will be improved by tuning this percentage are to be tested in the following section (Section 5.2).

\subsection{Neighborhood Impacts Calibration}

The task of exploring a "bottom-up" model's parameter space and discovering the impact of different parameter settings can be difficult and time-consuming. In this study, the spatial dynamic model's parameter space can be participated into two subsets: the set of parameters controlling the internal flows, and the set of parameters determining the neighborhood impacts. For the first set of parameters, they were calibrated and cross-validated by the Elastic Net model. For the second set of parameters $\left(P_{1}, P_{2}\right.$, and $\left.P_{3}\right)$, they were all arbitrarily set as 0.5 in Section 5.1. Although some evolutionary algorithms such as Genetic Algorithms (GAs) can be used to calibrate all the parameters in order to obtain a better prediction performance, only the second set of parameters were calibrated in this spatial dynamic model. The reasons for not using GAs to calibrate the first set parameters are fourfold: First, the parameters were already empirically estimated by the Elastic Net using the real data; Second, the definition of the utility was derived by the Elastic Net, and tuning the first set parameters will cause the linear combination of the socio-ecological variables represented by the utility no longer be the best predictors for population change; Third, although evolutionary algorithms such as GAs can reduce the computational time of running the model significantly in contrast with the exhaustive methods, the number of iterations required to find a solution in GAs remains unclear (Wright and Alajmi, 2005). Thus it is easier to test the convergences with fewer parameters. Last, GAs need tuning ranges of the parameters to identify their searching spaces for solutions, and unlike the second set of parameters which 
have a logical tuning range (form 0 to 1 ), the first set of parameter do not have tuning ranges. Applying GAs by creating tuning ranges from the empirically identified values by other data mining method (such as Elastic Net in this study) is redundant and trivial.

Thus, in order to find out the best percentage of the neighborhood impacts, the GA in this study was only applied to the second set of the parameters, with the fitness function defined as the total MSE of the prediction of the population and the developed land area percentage. By using this fitness function, it is assumed that the prediction accuracies of population and developed area percentage are of equal importance in determining the final values of the neighborhood impacts parameters. The tuning range of these parameters are set from zero to one. If the parameter equals to zero, it indicates that there are no direct neighborhood impacts, and if the parameter equals to one, it means that there is no autonomy or self-control. One hundred iterations were generated and the results of the GA are shown in Figure 5.4.

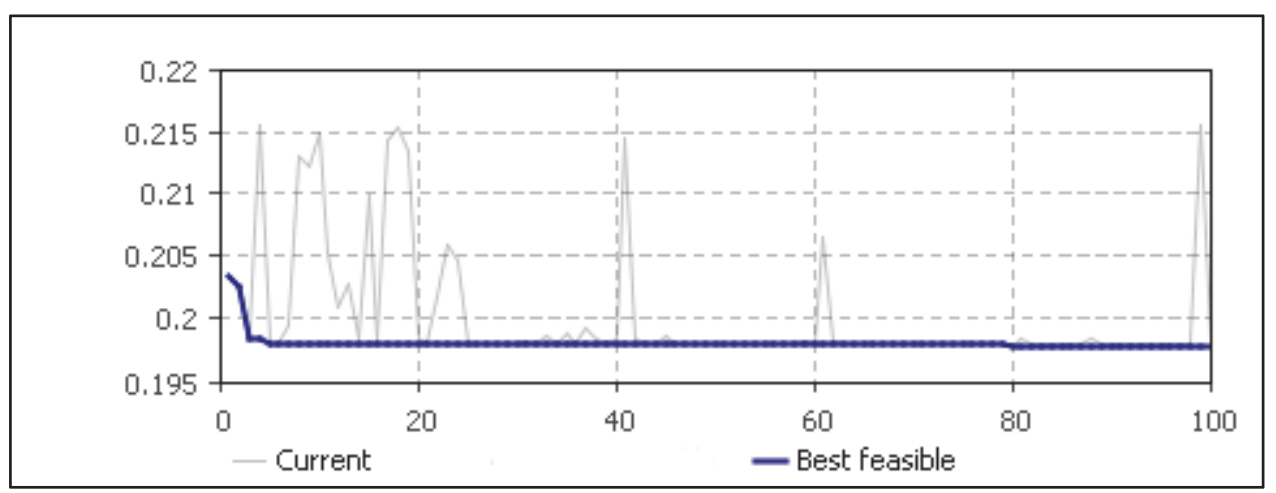

Figure 5.4 Genetic Algorithm Results for Calibrating the Neighborhood Impacts

In Figure 5.4, the grey line represents the MSE of each iteration, whereas the blue line represents the best chromosome (feasible solution) of each generation. From the figure, the best solution of each generation of the GA converges quickly after the first several generations. After the GA calibration, the sum of MSEs drops to 0.1978. The MSE for the population prediction is 0.1368 , and the MSE for the development land area prediction is 0.0609 . From the best solution picked, the contribution percentage of neighborhood impacts are $0 \%$ for 
population change $\left(P_{1}=0\right), 50 \%$ for development land area change $\left(P_{2}=0.5\right)$, and $0 \%$ for utility change $\left(P_{3}=0\right)$, which means that the best prediction accuracy was acquired when there was no direct neighborhood impacts for the utility change and the population change, and half neighborhood impacts and half autonomy for the developed land use area percentage change.

Although the MSEs were proven to be lowered, the calibrated results from the GA, with $P_{1}=0$ and $P_{3}=0$, are not satisfying results, because the emergence resulting from neighborhood feedbacks is one of the major focuses in this spatial dynamic model. In order to test the variations of the simulated results for the future predictions by using different neighborhood effects settings, an independent sensitivity analysis was conducted on $P_{1}, P_{2}$ and $P_{3}$ separately. The sensitivity analysis was conducted for the time period from 2010 to 2050 , which is the time period used for the "short time-period" simulations in the subsequent Chapters. $P_{1}, P_{2}$, and $P_{3}$ were incremented by 0.1 independently from 0 to 1 . The total population and the total developed area percentage of all the spatial units (standardized values) were documented every ten time steps (at the year of 2020, 2030, 2040 and 2050). Each simulation trial only had one of neighborhood parameters varied. The simulated total population time series and total developed land area time series corresponding to different values of $P_{1}, P_{2}$ and $P_{3}$ are shown in Figure 5.5. 


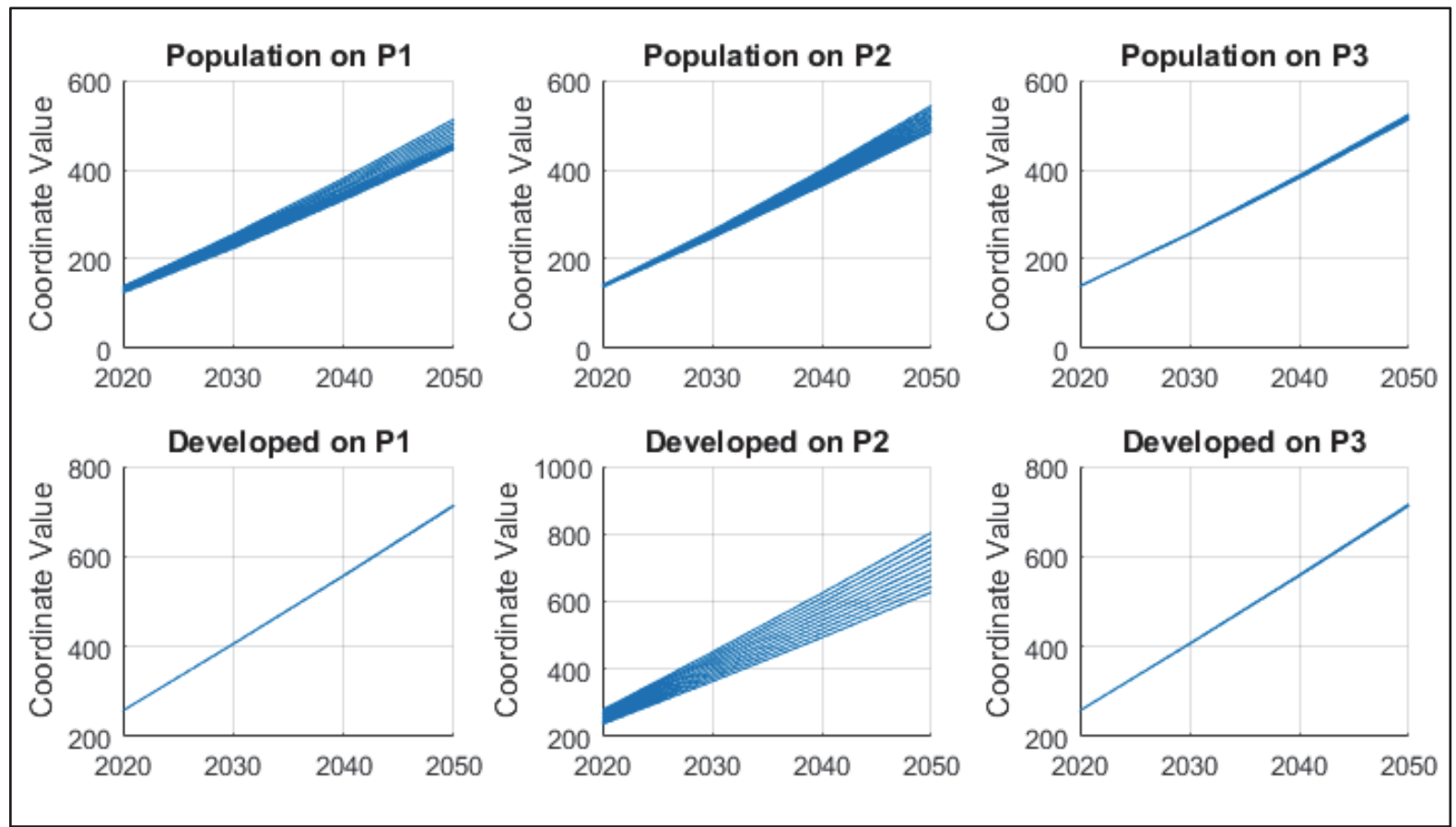

Figure 5.5 Total Population and Total Developed Area Percentage of all the Spatial Units (Standardized Values) in the Sensitivity Analysis of $\mathrm{P}_{1}, \mathrm{P}_{2}$, and $\mathrm{P}_{3}$

From the sensitivity analysis results in Figure 5.5, the total population does not have much variations with different $P_{3}$ values, which means that adding the direct neighborhood effects on utility changes does not make much difference on the final results. In contrast, $P_{1}$ and $P_{2}$ have higher impacts on the total population variations. The variations of the total population due to the changing values of $P_{1}$ and $P_{2}$ is increasing over time. This means that although $P_{2}$ does not directly add neighborhood impact on population changes, its indirect neighborhood feedbacks creates a lot variations on the final total populations. For the total developed area percentage, there are not much variations with different $P_{1}$ and $P_{3}$ values. It is concluded that adding the direct neighborhood effects on population and utility does not make any difference on the final results of developed area percentages. On the contrary, the variations created by different $P_{2}$ values are huge, which means that the direct neighborhood impact on developed area percentages cannot be ignored.

Thus, the predictive simulations showed little variations over the direct neighborhood effects for population changes and utility changes. The final model was determined to base on the results from the GA, since these values were claimed by the GA as most accurate in 
predicting the population and the developed area percentage together. The comparisons of the standardized prediction values by the final model and the standardized values of the real data are shown in Figure 5.9 and Figure 5.10.

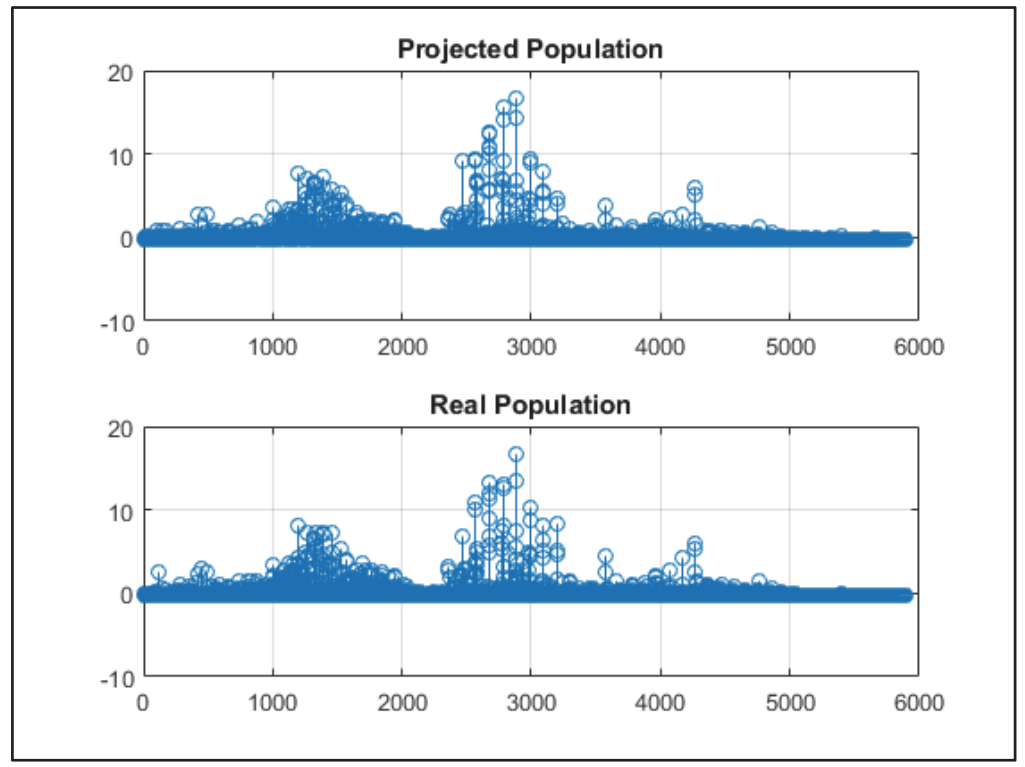

Figure 5.6 Comparison between the Predicted and the Real Population (Standardized) in 2010 by Spatial Units (after GA Calibration)

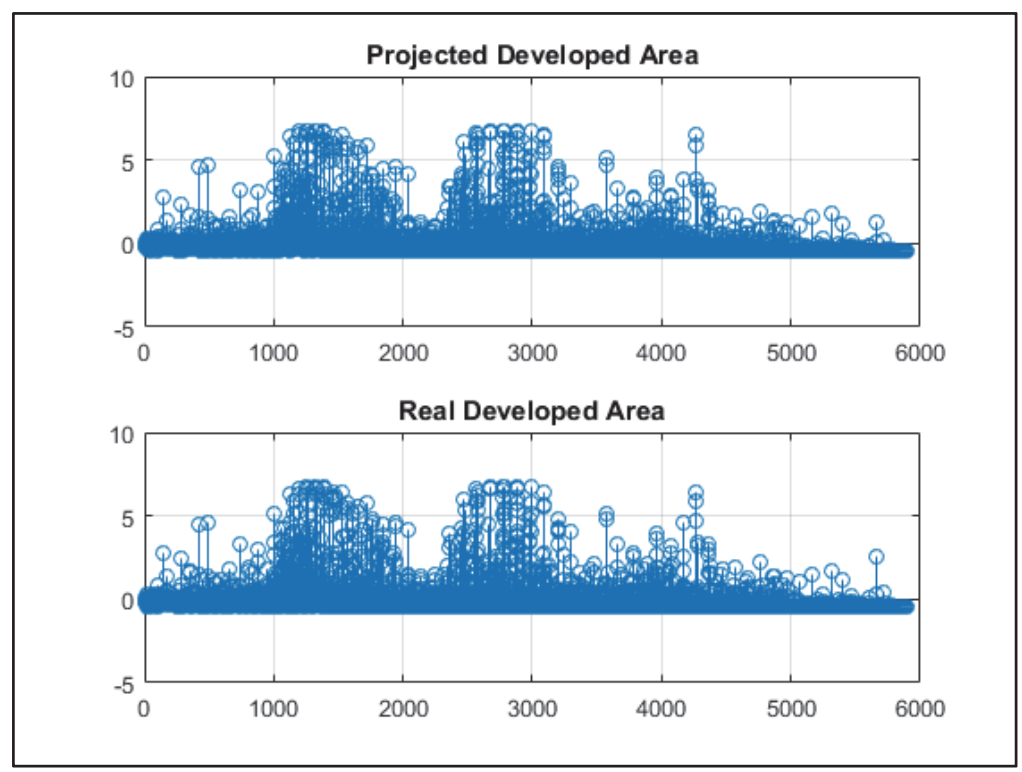

Figure 5.7 Comparison between the Predicted and the Real Developed Land Area Percentage (Standardized) in 2010 by Spatial Units (after GA Calibration)

The real population of the study area in 2010 is shown in Figure 5.8, and the projected population in 2010 is shown in Figure 5.9. The real developed land area percentage and the 
projected one by the spatial dynamic model after GA of the study area in 2010 are shown in Figure 5.10 and Figure 5.11 respectively.

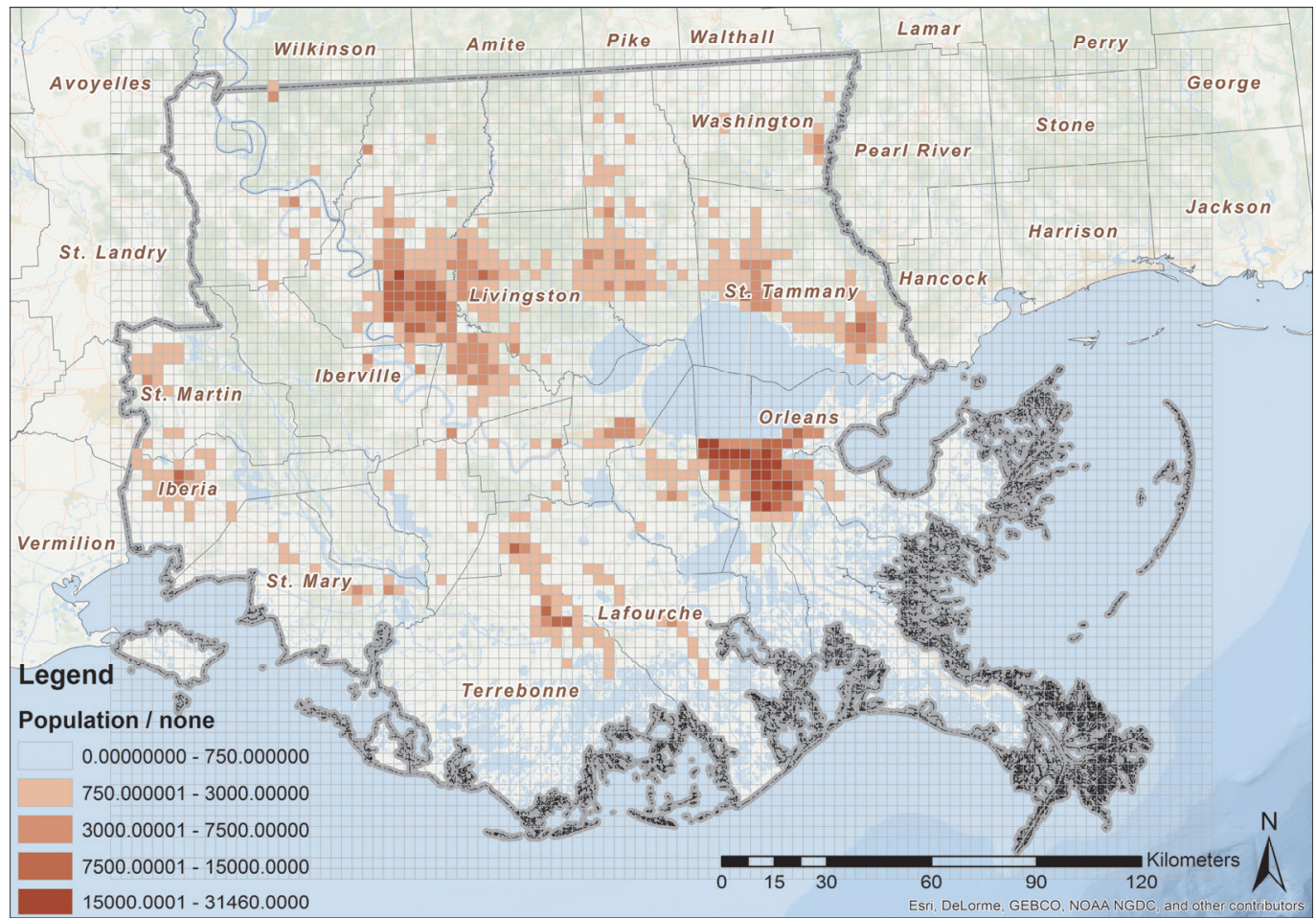

Figure 5.8 Real Population of the Study Area in 2010 


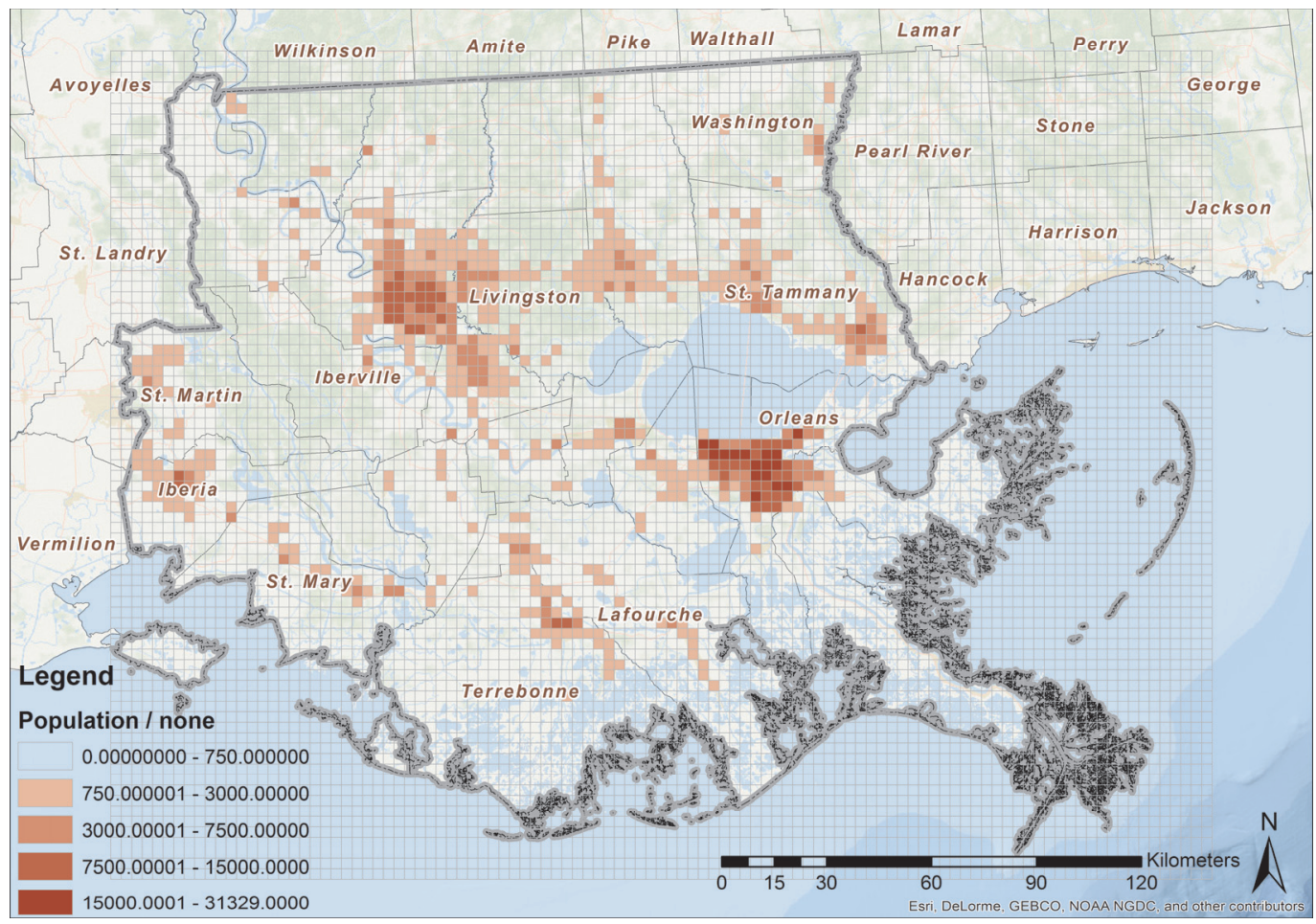

Figure 5.9 Population Projected by the Spatial Dynamic Model after Genetic Algorithms Calibration of the Study Area in 2010

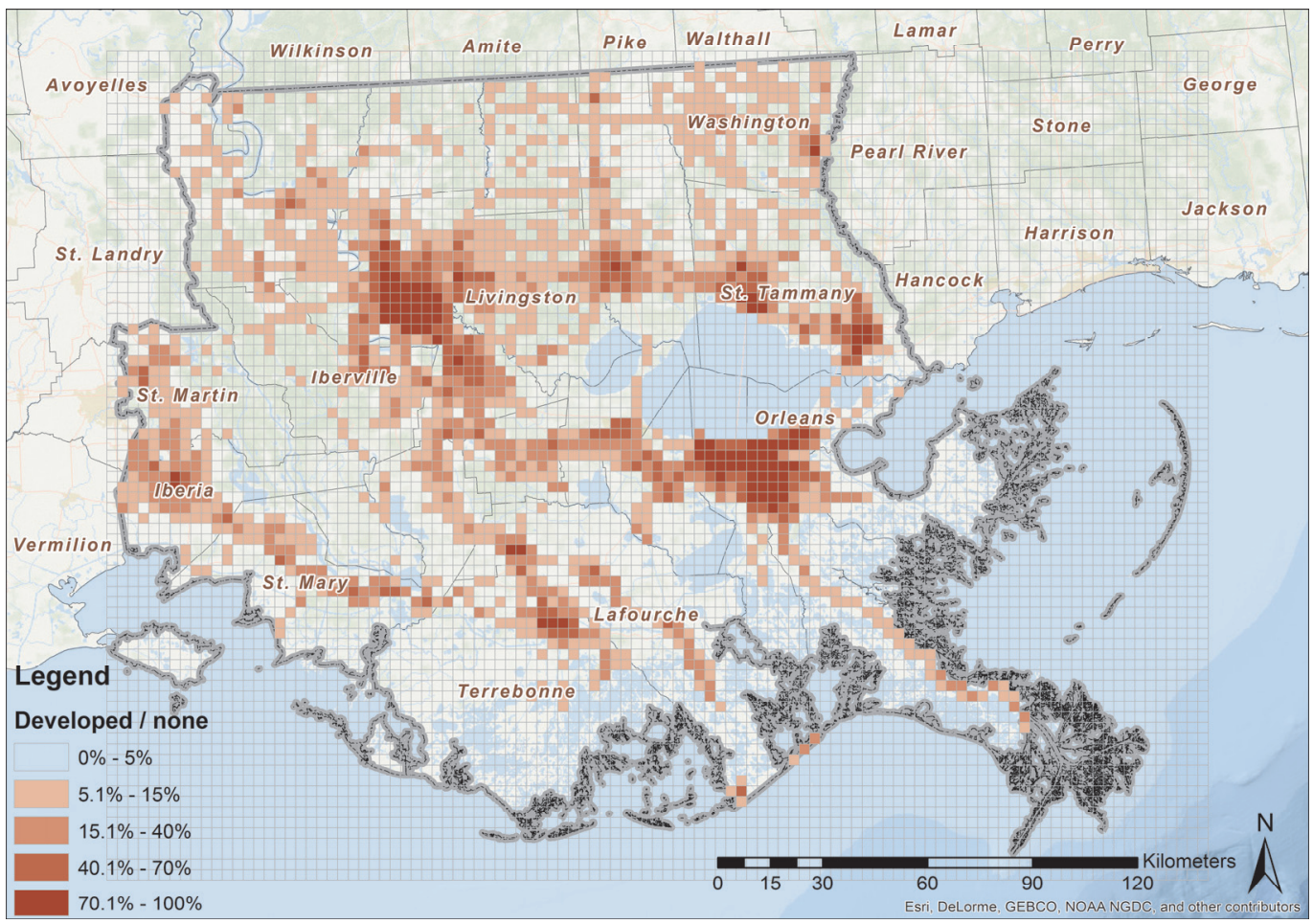

Figure 5.10 Real Developed Land Use Area Percentage of the Study Area in 2010 


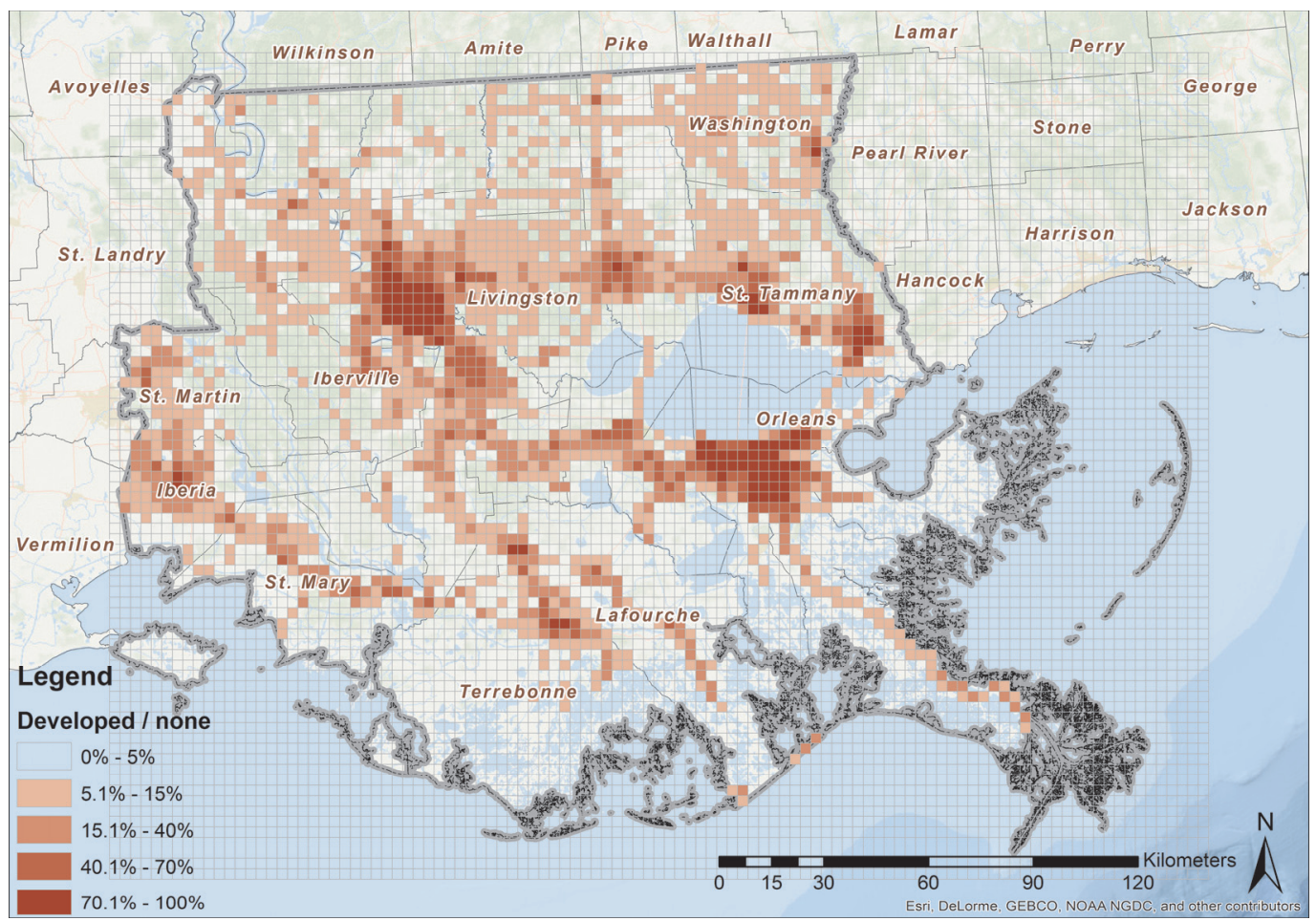

Figure 5.11 Developed Land Use Area Percentage Projected by the Spatial Dynamic Model after Genetic Algorithms Calibration of the Study Area in 2010

The MSE measurement used above reflects the fitness of the simulation results to the real data. It is an effective way to compare the accuracies among different modeling methods and parameters. However, its value range is dependent on the input data used, thus it is only comparable within this research. In order to offer some generally comparable accuracy information, confusion matrix measurement was used to indicate the prediction accuracy of the model from 2000 to 2010 . The simulated results of the populations and the developed area percentages of all the spatial units were classified into five groups, as well as the real data. The break values in the groupings were set manually using the "Jenks Natural Break" values of the real datasets of 2000 as references. Confusion matrixes were calculated for the population and the developed area percentage of 2010 between the simulated results and the real datasets, and they are shown in Table 5.1 and Table 5.2. 
Table 5.1 Confusion Matrix for Population Prediction of 2010

\begin{tabular}{|c|c|c|c|c|c|c|c|}
\hline & \multicolumn{5}{|c|}{ Real Population } & \multirow[b]{2}{*}{ Accuracy } \\
\hline & & $0-750$ & $750-300$ & $\begin{array}{l}3000- \\
7500\end{array}$ & $\begin{array}{l}7500- \\
15000\end{array}$ & $\begin{array}{l}15000- \\
32000\end{array}$ & \\
\hline \multirow{6}{*}{$\begin{array}{l}\text { Simulated } \\
\text { Population }\end{array}$} & $0-750$ & 5256 & 70 & 0 & 0 & 0 & $98.69 \%$ \\
\hline & $750-300$ & 48 & 314 & 12 & 0 & 0 & $83.96 \%$ \\
\hline & $3000-7500$ & 1 & 25 & 94 & 3 & 0 & $76.42 \%$ \\
\hline & $7500-15000$ & 0 & 0 & 5 & 43 & 2 & $86.00 \%$ \\
\hline & $15000-32000$ & 0 & 0 & 0 & 2 & 15 & $88.24 \%$ \\
\hline & Total & 5305 & 409 & 111 & 48 & 17 & $97.11 \%$ \\
\hline
\end{tabular}

Table 5.2 Confusion Matrix for Developed Area Percentage Prediction of 2010

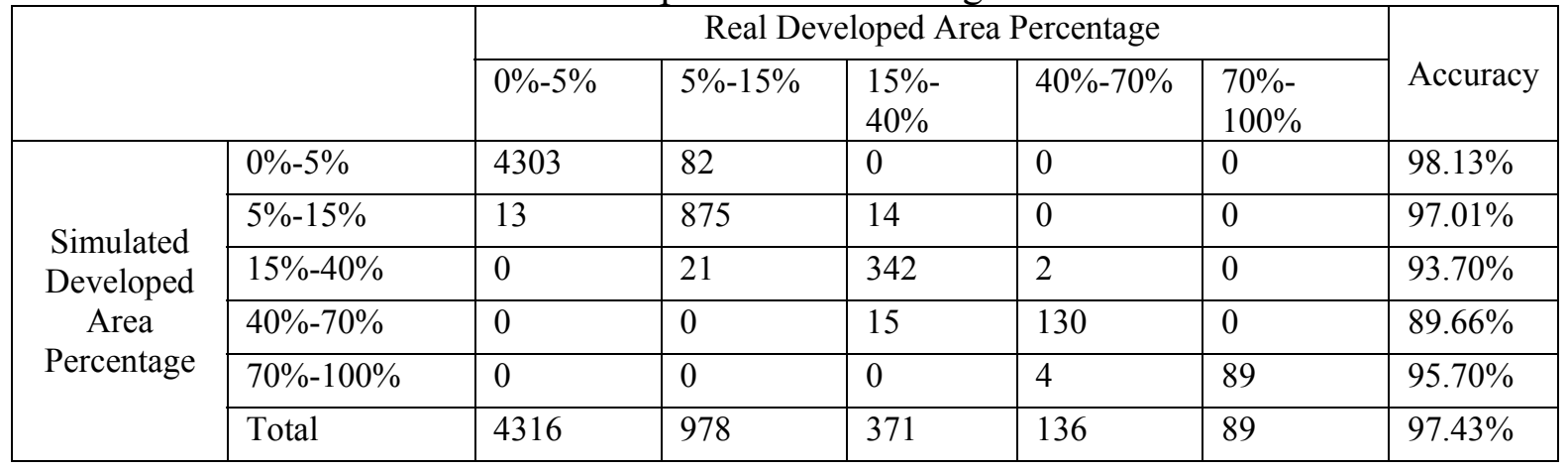

The total accuracies in both of the confusion matrixes are the weighted means of the accuracy of each group using the number of spatial units in each group from the simulated results as weight. From Table 5.1 and Table 5.2, the final spatial dynamic model claims an accuracy above $97 \%$ in both predicting the population and the developed area percentage from 2000 to 2010.

\subsection{Monte Carlo Uncertainty Analysis}

Monte Carlo probabilistic uncertainty analysis using simple random sampling (SRS), was applied to the spatial dynamic mode in this study to examine the propagation of uncertainty errors and identify the most important contributors to uncertainty (Doubilet et al., 1985). The uncertainty analysis was conducted on the simulation time period from 2010 to 2050, which corresponds to the "short time-period" animations discussed in the subsequent chapters. The total values of the three stock variables (population, developed area percentage and utility) over all the spatial units are the assessment variables of the uncertainty errors. Z-score values 
were used for the total values of these stock variables. The endpoints recording the values of the assessment variables were set at the $10^{\text {th }}, 20^{\text {th }}, 30^{\text {th }}$, and $40^{\text {th }}$ time step (every ten years untill the end of the simulations). The uncertainty parameters are all of the parameters listed in Table 4.4 , and the tuning ranges for these parameters were set as from $50 \%$ below their original values to $50 \%$ over their original values. The details of the tuning ranges for these parameters are in Table 5.3.

The uniform probability distribution was used for the Monte Carlo simulation to pick the values for these parameters within their tuning ranges. All the parameters were varied independently by Monte Carlo, and 500 trials was generated. For each trial, a random draw was made for each parameter from its distribution to obtain the value. The values of the assessment variables were documented during the running of each trial at the specified time steps. Histograms were created for the assessment variables from all the 500 trials at all the specified recording endpoints. All the histograms used 50 equal-size bins, and their centers of the bins are plotted in Figure 5.12, Figure 5.13, and Figure 5.14.

Table 5.3 Probability Distribution Specification for the Monte Carlo Uncertainty Analysis (Unstandardized Real Value)

\begin{tabular}{|c|r|r|r|c|}
\hline Parameters & \multicolumn{1}{|c|}{ Original } & \multicolumn{1}{l|}{ Min } & \multicolumn{1}{l|}{ Max } & Probability Distribution \\
\hline $\mathrm{a} 1$ & $2.19 \mathrm{E}-11$ & $1.10 \mathrm{E}-11$ & $3.29 \mathrm{E}-11$ & Uniform \\
\hline $\mathrm{b} 1$ & $8.90 \mathrm{E}-05$ & $4.45 \mathrm{E}-05$ & $1.33 \mathrm{E}-04$ & Uniform \\
\hline $\mathrm{c} 1$ & $2.86 \mathrm{E}-01$ & $1.43 \mathrm{E}-01$ & $4.30 \mathrm{E}-01$ & Uniform \\
\hline $\mathrm{d} 1$ & $-6.88 \mathrm{E}-02$ & $-1.03 \mathrm{E}-01$ & $-3.44 \mathrm{E}-02$ & Uniform \\
\hline $\mathrm{f} 1$ & $2.35 \mathrm{E}-07$ & $1.18 \mathrm{E}-07$ & $3.53 \mathrm{E}-07$ & Uniform \\
\hline $\mathrm{d} 2$ & $5.86 \mathrm{E}+01$ & $2.93 \mathrm{E}+01$ & $8.79 \mathrm{E}+01$ & Uniform \\
\hline $\mathrm{e} 2$ & $4.63 \mathrm{E}+01$ & $2.32 \mathrm{E}+01$ & $6.95 \mathrm{E}+01$ & Uniform \\
\hline $\mathrm{f} 2$ & $-9.25 \mathrm{E}-04$ & $-1.39 \mathrm{E}-03$ & $-4.62 \mathrm{E}-04$ & Uniform \\
\hline $\mathrm{g} 2$ & $-8.01 \mathrm{E}-01$ & $-1.20 \mathrm{E}+00$ & $-4.01 \mathrm{E}-01$ & Uniform \\
\hline $\mathrm{a} 3$ & $-3.35 \mathrm{E}-07$ & $-5.03 \mathrm{E}-07$ & $-1.68 \mathrm{E}-07$ & Uniform \\
\hline $\mathrm{b} 3$ & $6.01 \mathrm{E}-02$ & $3.00 \mathrm{E}-02$ & $9.01 \mathrm{E}-02$ & Uniform \\
\hline $\mathrm{d} 3$ & $2.62 \mathrm{E}+02$ & $1.31 \mathrm{E}+02$ & $3.92 \mathrm{E}+02$ & Uniform \\
\hline e3 & $2.57 \mathrm{E}+02$ & $1.29 \mathrm{E}+02$ & $3.86 \mathrm{E}+02$ & Uniform \\
\hline f3 & $-2.31 \mathrm{E}-02$ & $-3.46 \mathrm{E}-02$ & $-1.15 \mathrm{E}-02$ & Uniform \\
\hline g3 & $-1.92 \mathrm{E}-01$ & $-2.88 \mathrm{E}-01$ & $-9.61 \mathrm{E}-02$ & Uniform \\
\hline
\end{tabular}




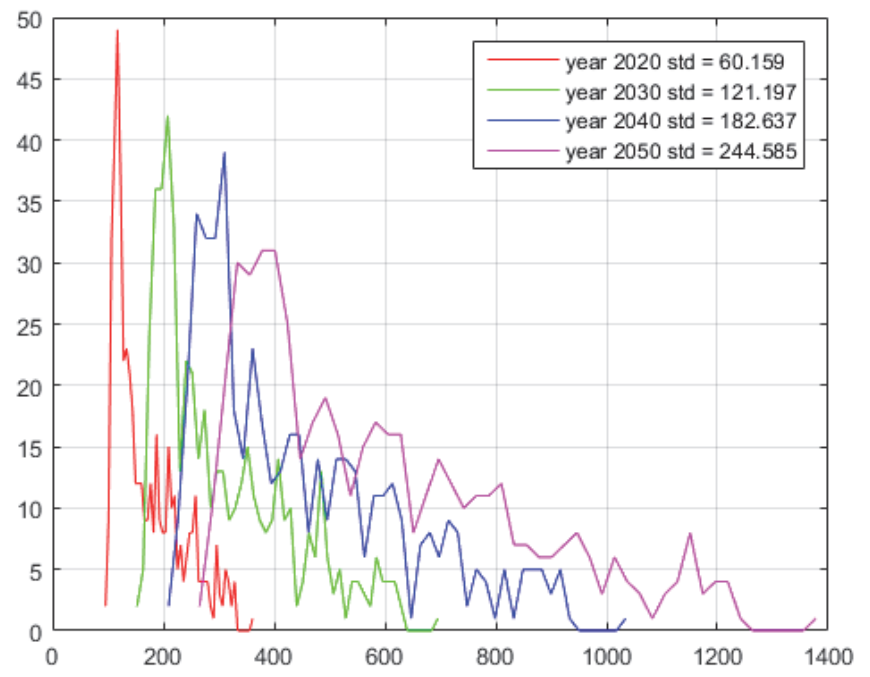

Figure 5.12 Bin-Centers for the Histograms of Total Population

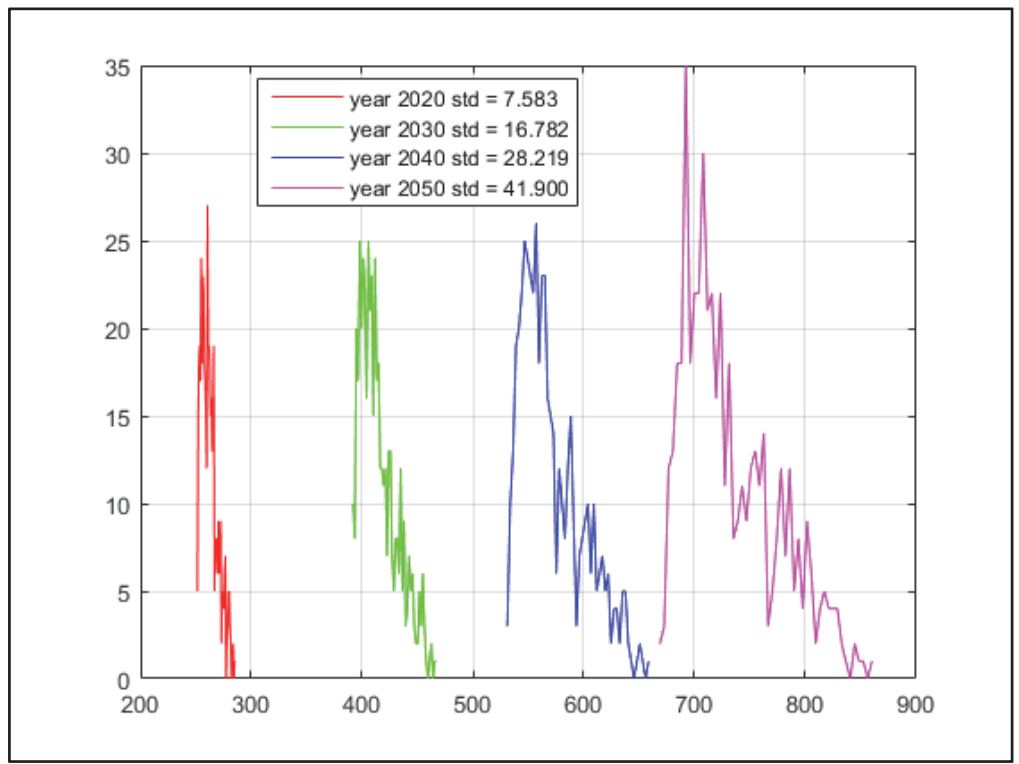

Figure 5.13 Bin-Centers for the Histograms of Total Developed Area Percentage 


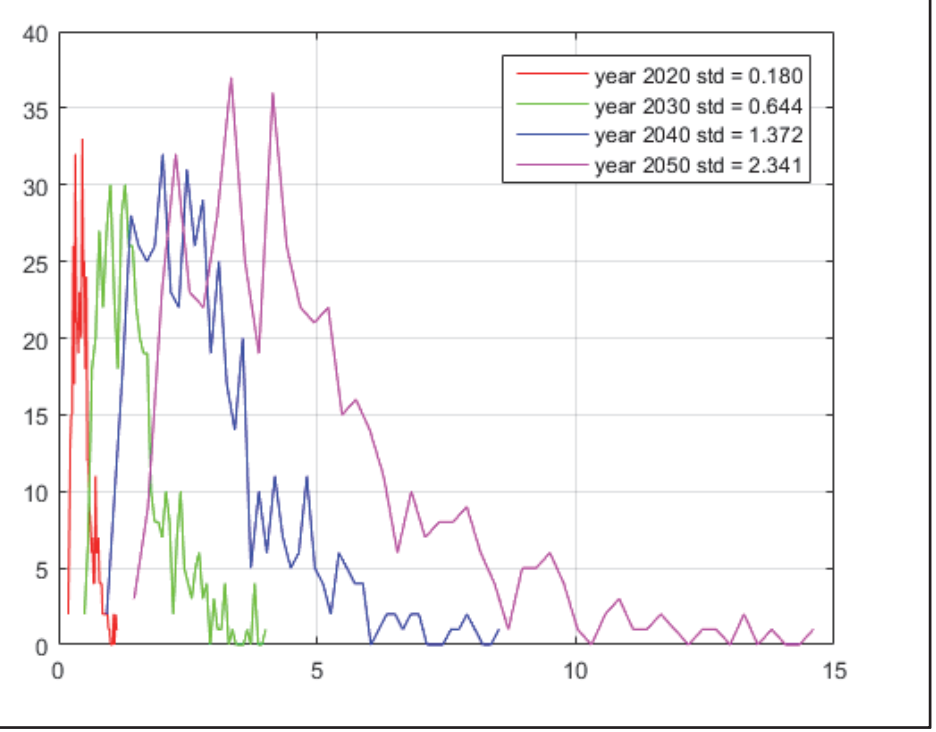

Figure 5.14 Bin-Centers for the Histograms of Total Utility

From Figure 5.12, Figure 5.13, and Figure 5.14, the uncertainties of the assessments propagated over every decades, and the longer the simulation the larger the uncertainty errors. It should be noted that the probability distributions of the simulated total population and the simulated total developed land area percentage are one-tailed to the right. The reason for this phenomena is probably the constraints added in the simulation settings (mentioned in Chapter 3). In contrast, the probability distribution of the simulated total utility is more like two tailed. The propagated uncertainties represented by the standard deviations of the probability distributions for total population and total developed land area percentage (The values are in the legends of Figure 5.12, 5.13, and 5.14) increased almost linearly over simulation time. For the total utility, the uncertainty increased dramatically from the first record endpoint to the second (2020 to 2030), and after that, the increasing speed was also almost linear over simulation time.

Pearson correlation confidents between the assessment variables and the parameters were used to reveal the approximate relative contribution of each parameter to the variance of each assessment. The parameters having the greatest effects are considered to be the 
parameters with the highest correlation coefficients. The color graphs showing these correlation details are in Appendix B1, B2, B3, and B4. The selected parameters with the highest correlation coefficients with the assessment variables are summarized in Table 5.4.

Table 5.4 Pearson Correlations between the Assessment Variables and the Selected Parameters

\begin{tabular}{|c|c|r|r|r|r|}
\hline \multirow{2}{*}{$\begin{array}{c}\text { Assessment } \\
\text { Variable }\end{array}$} & \multirow{2}{*}{ Parameter } & \multicolumn{4}{|c|}{ Pearson Correlation Coefficient } \\
\cline { 2 - 6 } & & Year 2020 & Year 2030 & Year 2040 & \multicolumn{1}{|c|}{ Year 2050 } \\
\hline \multirow{3}{*}{ Total Population } & $\mathrm{e}_{3}$ & 0.78 & 0.77 & 0.77 & 0.76 \\
\cline { 2 - 6 } & $\mathrm{f}_{3}$ & 0.47 & 0.5 & 0.52 & 0.54 \\
\hline \multirow{2}{*}{$\begin{array}{c}\text { Total Developed } \\
\text { Area Percentage }\end{array}$} & $\mathrm{d}_{2}$ & 0.41 & 0.36 & 0.32 & 0.3 \\
\cline { 2 - 6 } & $\mathrm{e}_{2}$ & 0.76 & 0.8 & 0.83 & 0.85 \\
\hline \multirow{3}{*}{ Total Utility } & $\mathrm{f}_{1}$ & 0.68 & 0.61 & 0.58 & 0.57 \\
\cline { 2 - 6 } & $\mathrm{e}_{3}$ & 0.52 & 0.56 & 0.57 & 0.57 \\
\cline { 2 - 6 } & $\mathrm{f}_{3}$ & 0.32 & 0.37 & 0.39 & 0.4 \\
\hline
\end{tabular}

The parameters in Table 5.4 are identified as high-sensitivity parameters, and reducing their variations should reduce the amount of overall uncertainty in the final results. From Table 5.4 , it is also found that the correlation coefficients between these parameters and the assessments do not change much over time, thus their sensitivities do not propagate over time. The scatter plots between these variables and the assessment variables are in Appendix B (from B5 to B16). 


\subsection{References}

Boccara, N., Cheong, K. (1992). Automata network SIR models for the spread of infectious diseases in populations of moving individuals, J. Phys. A: Math. Gen. Journal of Physics A: Mathematical and General 25: 2447-2461.

Boccara, N., Cheong, K. (1993). Critical behaviour of a probabilistic automata network SIS model for the spread of an infectious disease in a population of moving individuals, $J$. Phys. A: Math. Gen. Journal of Physics A: Mathematical and General 26: 3707-3717.

Doubilet, P., Begg, C., Weinstein, M., Braun, P., Mcneil, B. (1985). Probabilistic Sensitivity Analysis Using Monte Carlo Simulation: A Practical Approach. Medical Decision Making 157-177.

Grimm, V., Berger, U., Bastiansen, F., Eliassen, S., Ginot, V., Giske, J., Goss-Custard, J., Grand, T., Heinz, S.K., Huse, G. (2006). A standard protocol for describing individualbased and agent-based models. Ecological Modelling 198(1-2): 115-126.

Parker, D. C., Berger, T., Manson, S. M. (2001). Agent-based models of land-use and land cover change. Report and Review of an International Workshop. Irvine, California, USA.

Wright, J.A., Alajmi, A.F. (2005). The robustness of genetic algorithms in solving unconstrained building optimization problems, 9th Int. IBPSA Building Simulation Conference, International Building Performance Simulation Association. 


\section{CHAPTER 6 : RESILIENCE AND SUSTAINABILITY ASSESSMENT}

The resilience assessment in this study utilizes the framework of the Resilience Inference Measurement (RIM) model, but instead of just analyzing the population changes, the whole simulated time series of the population were analyzed. Self-Organizing Map (SOM) was used to cluster the predicted population time series into unsupervised resilience groups using the distance measurement by the Short Time Series Distance (STSD). The sustainability of this study area was analyzed according to a set of sustainability criteria (goals), a hypothetically anticipated level of property damages caused by coastal nature hazards, and an amount of resources for mitigation planning. The sustainability was compared between the low resilience areas and the high resilience areas. The cost to maintain the low resilience areas in terms of the population count was also estimated.

\subsection{Resilience Assessment and Self-Organizing Map}

In terms of the relationship between resilience and sustainability of this coastal area, resilience is considered a short term expression and a prerequisite of sustainability, and it needs to be assessed with a justified methodology. The resilience assessment in this research utilizes the framework from the RIM model: using the population count of each spatial unit as the indicator of an equilibrium state, and using the population changes as the indicator to determine if the spatial units have the ability to stay around their equilibrium states. A detailed description of the RIM model can be found in a number of publications reviewed in Chapter 2 (Li, 2011; Lam et al., $2015 \mathrm{a \& b}$; Li et al., 2015). The variable of property damages from the coastal natural hazards was used to indicate the outside disturbances. However, for the indicator of the "recovery" dimension, instead of only using the population changes between the start date and

end date as in the RIM model, the whole simulated time series of population were analyzed. 
Self-Organizing Map (SOM) was used instead of K-means (the clustering method used in the RIM model) to put the spatial units into unsupervised resilience groups. The central time series of each group was analyzed to determine its resilience capacity.

\subsubsection{Self-Organizing Map Clustering}

The K-means algorithm used in the RIM model is one of the most widely used clustering techniques in solving Geographic Information Sciences (GISc) problems. However, the initialization procedure that ultimately determines which part of the solution space will be searched is one of the most important issues in the correct use of K-means. SOM as a substitute for K-means clustering was used in this study. Bação (2005) analyzed the performance of the SOM and K-means in the clustering problems of four different selected datasets (two realworld datasets and two synthetic datasets). The two real-world datasets were the well-known iris dataset (Fisher, 1936) and sonar dataset (Sejnowski and Gorman, 1988). In his study, the overall performances of SOM and K-means were compared by the criteria of the average quadratic error, standard deviation, average classification error, and average structural error on over 100 independent initializations. His conclusion was that the SOM is less prone to be limited in local optima than the K-means, and is more robust.

The basic idea of a SOM is to map the data patterns onto an $n$-dimensional grid of neurons or units ( $n$ is usually 1-dimension or 2-dimension to allow easy visualization). That grid space is known as the output space, as opposed to the input space where the data are originally in. Topological relations are preserved in this mapping. Patterns that are close in the input space will be mapped to units that are close in the output space, and vice-versa. A neighborhood function of distance decay is defined (in the output space) for the interactions between different units. During training, the radius of this function will usually decrease, so that each unit will become more isolated from the effects of its faraway neighbors. Many implementations of SOM decrease this radius to 1, which means that even in the final stages of training, each unit will at least be affected by its nearest neighbors, while a few 
implementations allow this parameter to decrease to zero. It should be noted that the SOM and the K-means algorithms are basically identical when the radius of the neighborhood function in the SOM equals zero (Bodt et al., 1997). SOMs can be used in many different ways, including the clustering tasks (Bação et al., 2005). In the clustering task of this study, each SOM unit is assumed as a cluster center, and thus a k-unit SOM will perform a task similar to a K-means.

\subsubsection{Self-Organizing Map Results}

Simulation using the final spatial dynamic model calibrated by GA in Chapter 5 was run from the year 2010 to the year 2050 using the real data of the year 2010 as the initial conditions. In this simulation, the assumption was that all the environmental factors remain the same as the historical data from the year 2000 to the year 2010, including the open water area growth rate, yearly subsidence rate, mean elevation, and the yearly property damages from coastal hazards. The projected population time series were then input into the SOM. It should be noted that for the clustering task of time series, a proper measurement of the distance between time series should be selected. In order to focus on the closeness of the shapes of the time series, "Short Time Series Distance" (STS) measurement was used in this study. Möller et al. (2003) proposed the use of STS distance measurement as the sum of the squared differences of the slopes in the two time series being compared. Mathematically, the STS distance between two time series is defined as Equation 6.1:

$$
d_{S T S}=\sqrt{\sum_{k=1}^{p}\left(\frac{x_{k+1}-x_{k}}{t_{k+1}-t_{k}}-\frac{v_{k+1}-v_{k}}{t_{k+1}-t_{k}}\right)^{2}}
$$

where $x$ and $v$ denote the two time series, $t_{k}$ is the time point for data point $x_{k}$ and $\mathrm{v} k$, and $p$ is the total number of time steps. To remove the effect of different magnitudes of the time series, 
$z$ score standardization of the time series was used in this study. Only the time series of the urban areas were analyzed, which are defined by the city boundaries of 2010 acquired from the U.S Census Bureau (totally 901 spatial units), because the rest of the areas had very low populations. Four resilience groups were chosen according to the RIM model, and the clustering results of the SOM are shown in the following figures (Figure 6.1, Figure 6.2, and Figure 6.3).

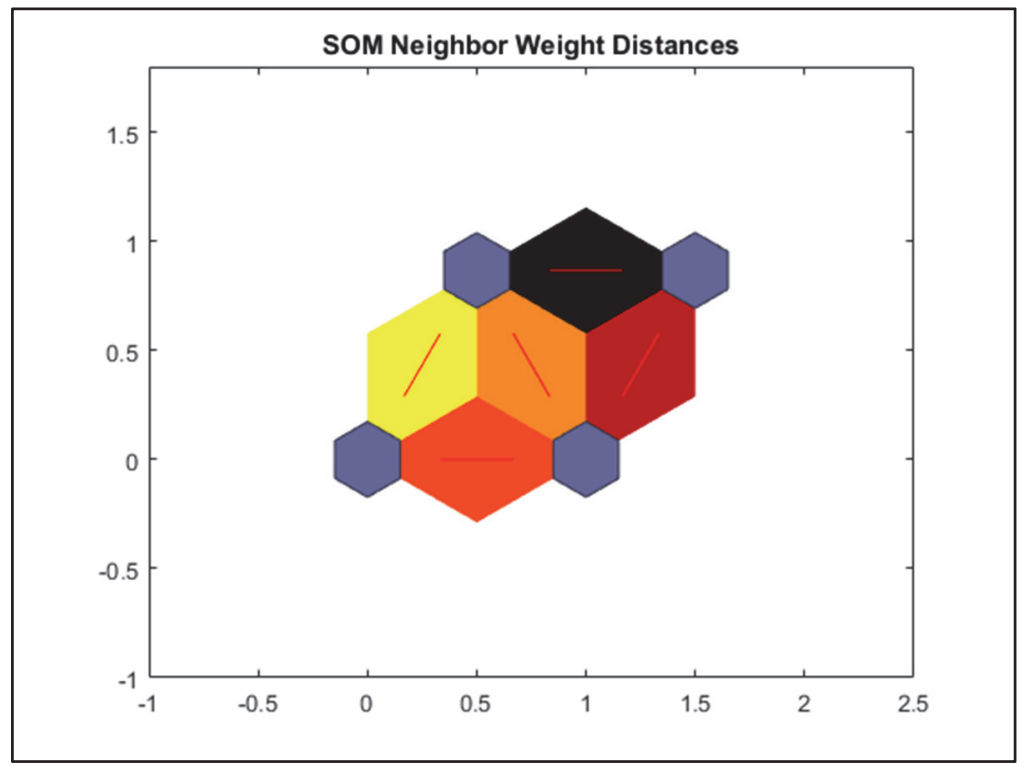

Figure 6.1 Neighbor Weight Distances of the Neurons in the Self-Organizing Map

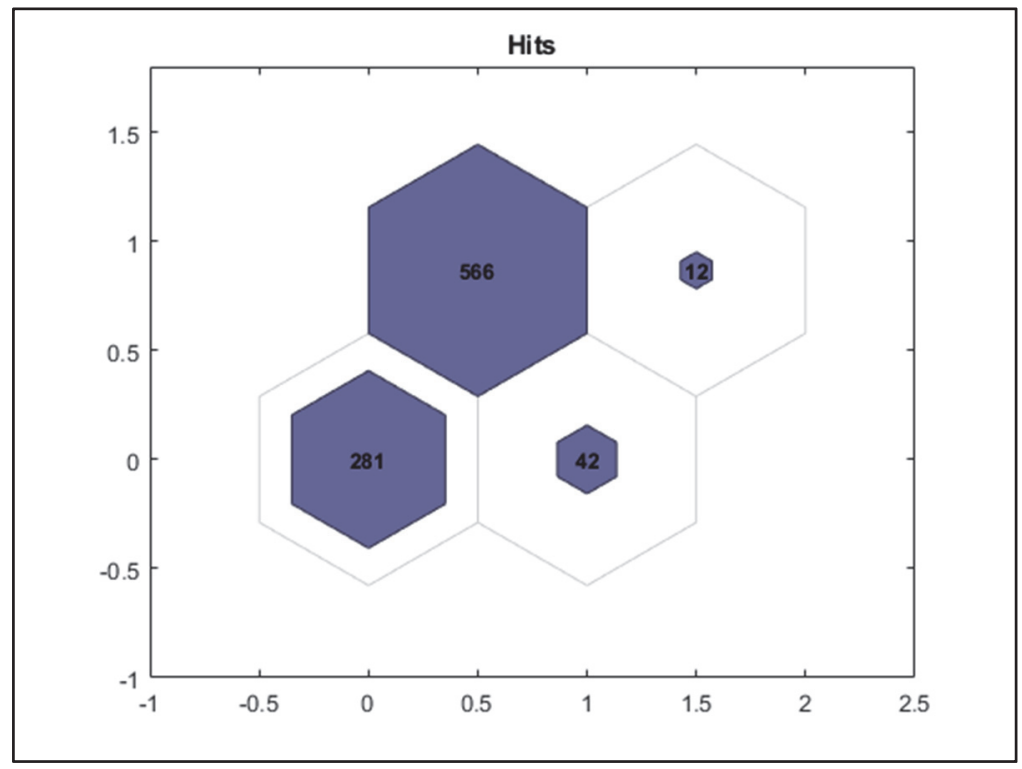

Figure 6.2 Number of the Spatial Units belonged to Each Neuron 


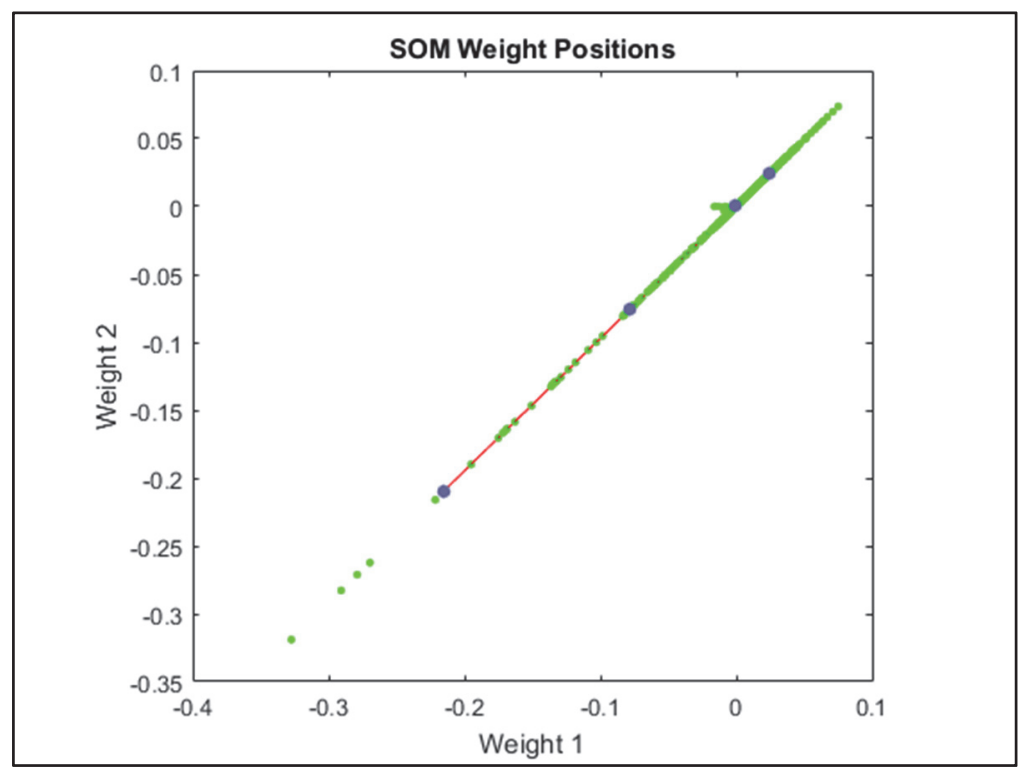

Figure 6.3 Weight Positions of the Spatial Units and the Four Neurons in the Output Space of the Self-Organizing Map

Figure 6.1 shows the neighbor weight distances among the four neurons. The four grey hexagons represent the neurons, and the red lines between each two neighboring neurons represent their closeness. Each red line has its background filled by a color with certain darkness. The darker the color is, the larger the distance, and the lighter the color the smaller the distance. Figure 6.2 shows how many spatial units each neuron consists of. Figure 6.3 maps the SOM weight positions of the 901 urban spatial units as well as the positions of the four neurons in the output space. Each neuron represents a cluster center. The closeness of the spatial units to their cluster centers in the output space are shown. In order to analyze the resilience, the central time series was extracted from the clusters, and the results are shown in Figure 6.4 . 


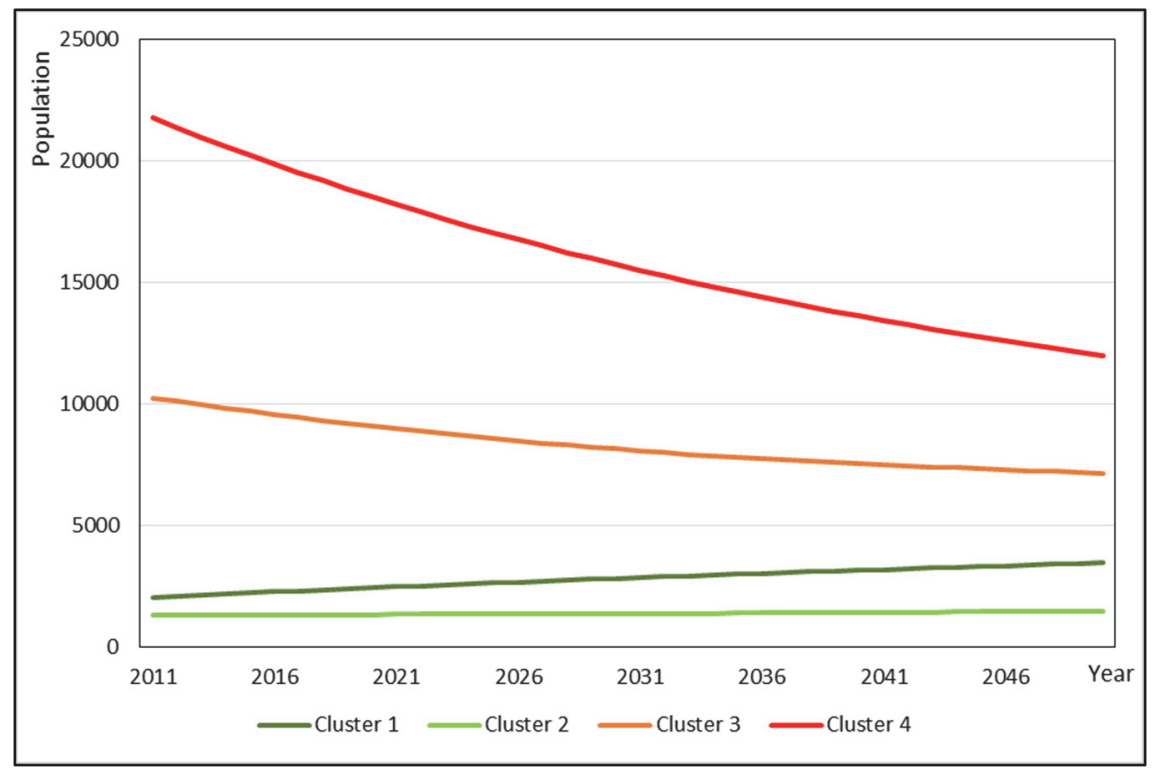

Figure 6.4 The Central Time Series for the Four Neurons (Clusters)

As shown in Figure 6.4, the populations of cluster 1 and cluster 2 are increasing over time throughout the whole simulation, whereas the populations of cluster 3 and cluster 4 are continuously decreasing, though their decreasing speed slows down in the later time steps of the simulation. The spatial units belonged to the four groups are mapped in Figure 6.5.

Four resilience states (usurper, resistant, recovering, and susceptible) used in the RIM model were used to name the four groups according to their central population changes time series. Group 1 (the usurper group), has the largest population growth speed. Group 2 (the resistant group), is very similar and quite closed to Group 1 (in the SOM output space), with its initial average population and its average population growing speed being lower than group 1. Most of the spatial units in this group are at the peripheral areas around the main city areas, and they also have smaller number of people. Group 3 (the recovering group), has a high initial population and a high population decreasing speed. Group 4 (the susceptible group), is like an extreme version of group 3, and has the highest initial population but also the highest decreasing speed. Spatial units in group 3 and group 4 are mostly located in the New Orleans area and the downtown area in Northern Baton Rouge. 


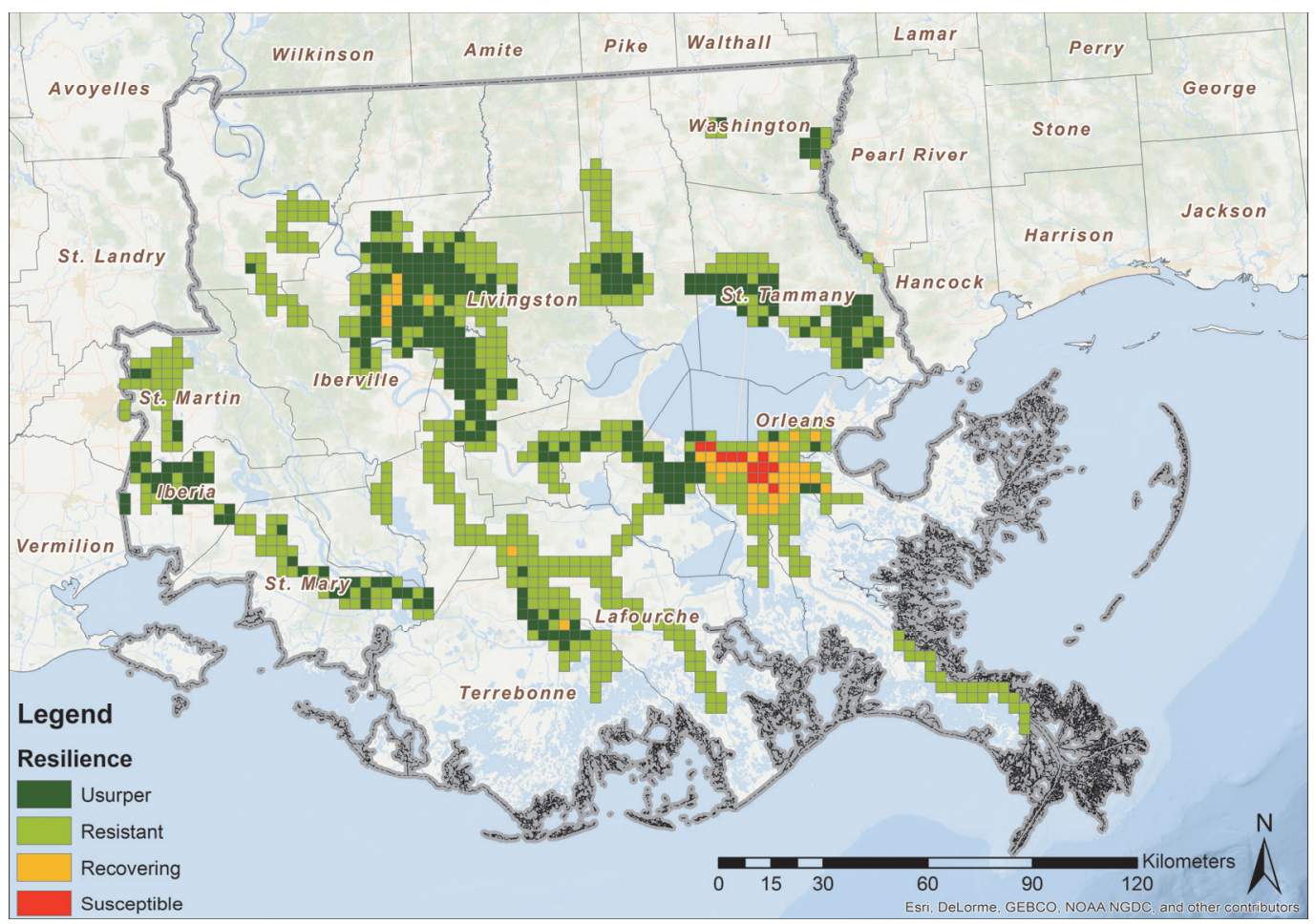

Figure 6.5 The Resilience Groups for all the Spatial Units in the City Areas of the Study Area

\subsection{Sustainability Analysis}

In this study, the sustainability of a region is considered a long term response of the resilience capacity in this study, and is assessed according to different specific criteria (goals). The sustainability criterion of a spatial unit is whether this spatial unit is able to maintain a certain percentage of its initial population (the sustainability goal) after several decades under exposure to coastal natural hazards. For example, if the sustainability goal is to maintain $80 \%$ of the initial population, then all the spatial units in the usurper and resistant groups are considered sustainable, since all of them not only are able to maintain this percentage of their initial population, but also are projected to have their populations increased in future years.

\subsubsection{Sustainability of the Low-Resilience Areas}

The susceptible and recovering spatial units are identified as the low-resilience spatial units. For these low-resilience spatial units, their final projected populations of the year 2050 are about $59 \%$ of their initial populations on average. Thus if the sustainability goal is to maintain a percentage lower than $59 \%$ of their initial populations by the year 2050 , these spatial 
units are sustainable. However, if the sustainability goal is to maintain a percentage more than $59 \%$ of their initial population, these spatial units are not sustainable. It should be noted that most of the low-resilience spatial units are located in the central urban areas of New Orleans (Figure 6.5), and these low resilience areas typically have much higher property damages, and much lower utility than others (Figure 6.6 and Figure 6.7).

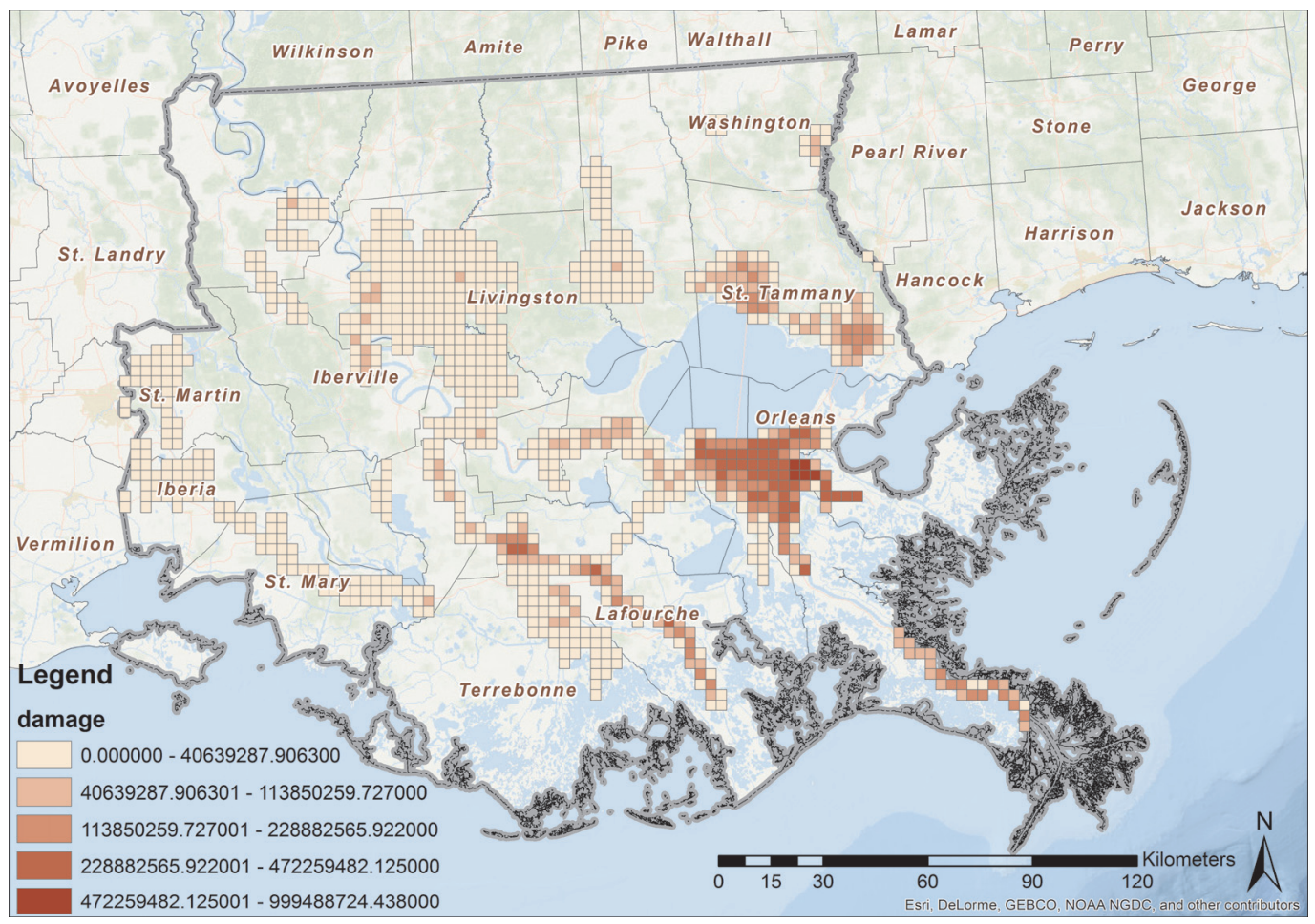

Figure 6.6 The Property Damages by Coastal Natural Hazards (Dollars) of the Spatial Units in the City Areas of the Study Area 


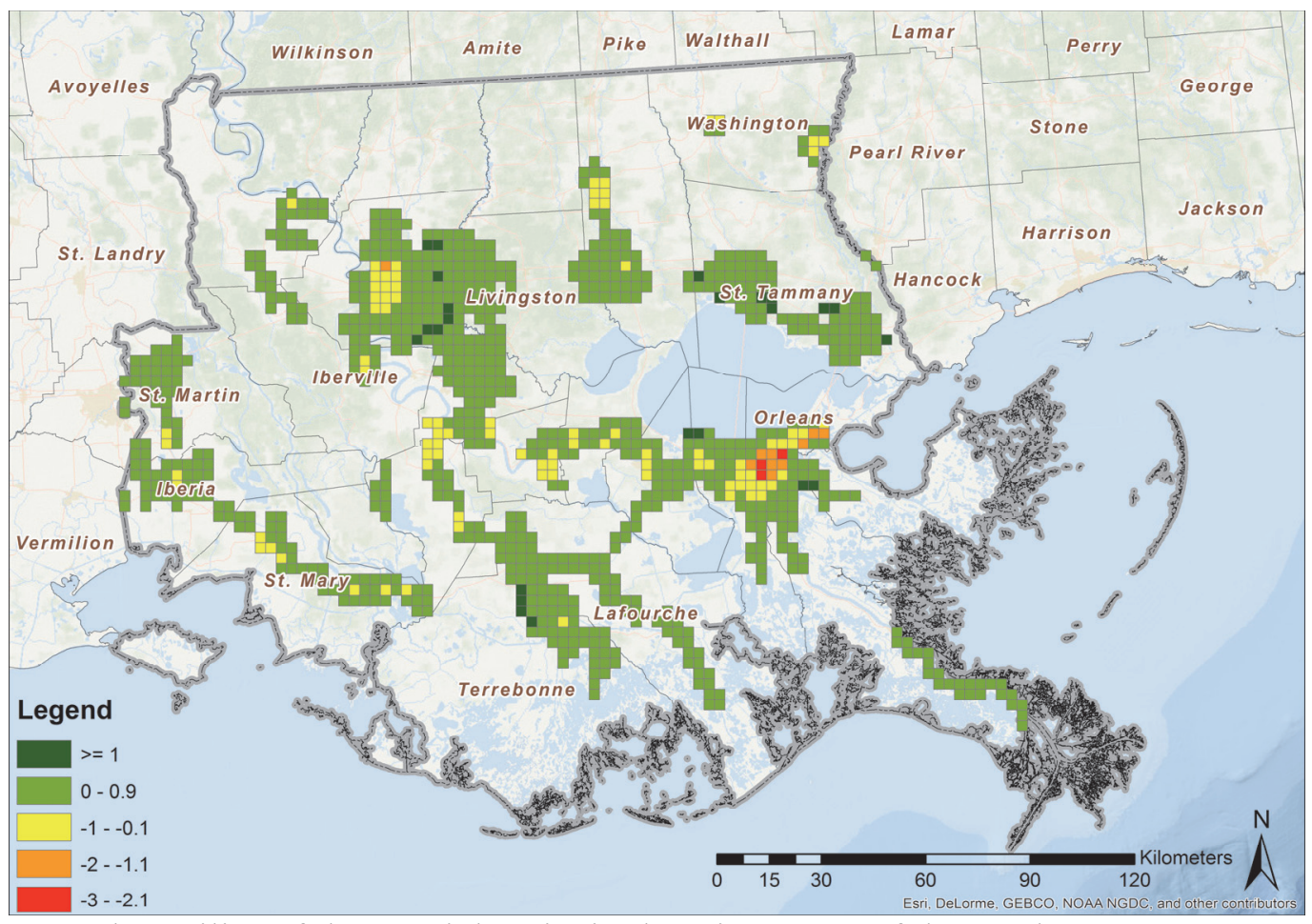

Figure 6.7 The Utility of the Spatial Units in the City Areas of the Study Area

As mentioned above, the simulation from which the resilience clustering results were extracted was run under the assumption that all the environmental factors are the same as the historical data from 2000 to 2010 (especially the property damages), thus the above "nonsustainable" conclusion can only be made under the condition that such assumption stands. In other words, these areas are not competent for a sustainability goal of maintaining a percentage higher than $59 \%$ of their initial populations, assuming that they keep suffering at least the same level of coastal natural hazards as they did from 2000 to 2010 . The objectives in the next two sub-sections are to find out: first, under which coastal hazards exposure scenarios that these spatial units will become sustainable; second, under what circumstances that these areas are mitigatable, and what is the cost of the mitigation. Here, mitigation means helping the population maintain the sustainability goal by using expenses. 


\subsubsection{Scenarios of Exposures}

To test the response of the low-resilience spatial units to different hypothesized amounts of property damages, a shrinkage ratio $\theta$ is defined in Equation 6.2. For each spatial unit, the deviation of its yearly damages from the mean in the future simulation will be the deviation of its yearly damages from the mean in the real data from year 2000 to 2010 multiplied by $\theta$.

$$
\theta=\left(u_{i}-\bar{u}\right) /\left(v_{i}-\bar{v}\right)
$$

where, $u_{i}$ denotes the yearly damages used in the predicting simulation for spatial unit $i, \bar{u}$ is the mean of $u_{i}, v_{i}$ denotes the real historical yearly damages spatial unit $i$ suffered from the year 2000 to the year 2010, and $\bar{v}$ is the mean of $v_{i}$. All the simulations are under the assumption that the mean yearly damages of all the spatial units in the simulation equal to their mean yearly damages of the historical data from 2000 to $2010(\bar{u}=\bar{v})$. For all the hypothesized hazards scenarios created in this way, it is also assumed that the property damages distribution is the same as the historical data, which means that the spatial units that were highly exposed to property damages will still have high property damages in the simulation, and vice versa. When $\theta$ equals 1 , the yearly damages for each spatial unit used in the simulation exactly equals their historical yearly damages. When $\theta$ is greater than 1 , the yearly damages for each spatial unit used in the simulation is more deviated from the mean than the real historical records. For the low-resilience spatial units, this means that they suffer more damages. When $\theta$ is smaller than 1 , the yearly damages for each spatial unit used in the simulation is closer to the mean than the real historical records. For the low-resilience spatial units, this means that they suffer less damages. At an extreme situation when $\theta$ equals 0 , the yearly damages for all the spatial units used in the simulation are the same and equals their mean. Several simulations with $\theta$ values ranged from 0 to 3 were tested, and the average populations of all the low resilience spatial units are monitored and documented at the ends of the simulations (Figure 6.8). 


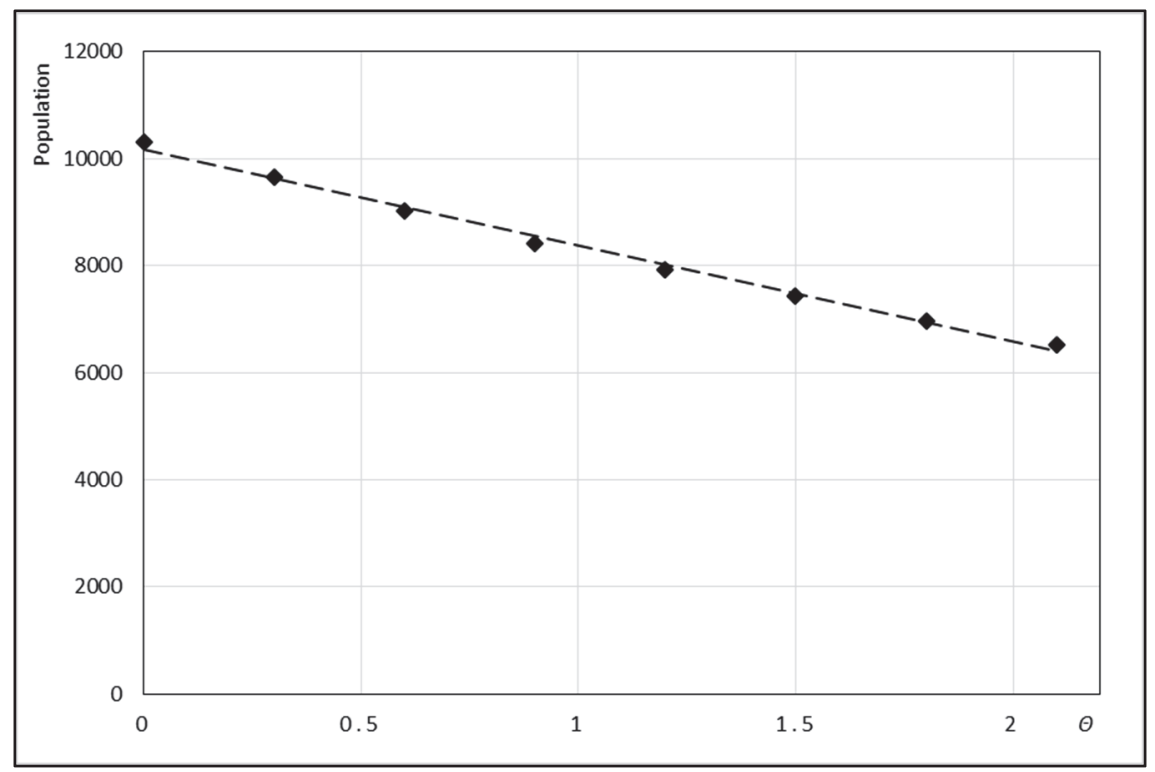

Figure 6.8 Average Population of the Low-Resilience Spatial Units Simulated with Different $\theta$ Values

A monotonous and almost liner relationship was found between the $\theta$ value and the final average population, and the higher the $\theta$ value, the lower the final average population. Thus if the $\theta$ value is smaller than one, these susceptible spatial units are able to achieve the sustainability goal with a higher future population projected.

\subsubsection{Mitigations}

Since the unpredictable coastal hazards have especially high possibility of occurrence in this vulnerable coastal area, mitigation plans are of extreme importance in this study. Here, mitigation refers to any measures that can reduce the damages from coastal hazards. To test the effects of different budgets of mitigation plans, it is assumed that each dollar in the mitigation expenses has the same power to increase the population as the power of each dollar in the property damages to decrease the population. For example, considering a spatial unit having a property damage of $m$ dollars and a mitigation expense of $n$ dollars, the projected population is $p_{1}$ when $m$ equals 0 and $n$ equals 0 , the simulated population is $p_{2}$ when $m$ equals $c$ (a certain amount of money) and $n$ equals 0 , and the simulated population is $p_{3}$ when $m$ equals 
0 and $n$ equals $c$, the following set of equations is established according to the above assumption.

$$
\left\{\begin{aligned}
p_{1} & >p_{2} \\
p_{3} & >p_{1} \\
p_{1}-p_{2} & =p_{3}-p_{1}
\end{aligned}\right.
$$

Several simulations with different amounts of mitigation expenses were tested. Figure 6.9 shows the average populations of the low-resilience spatial units at the end of the simulations.

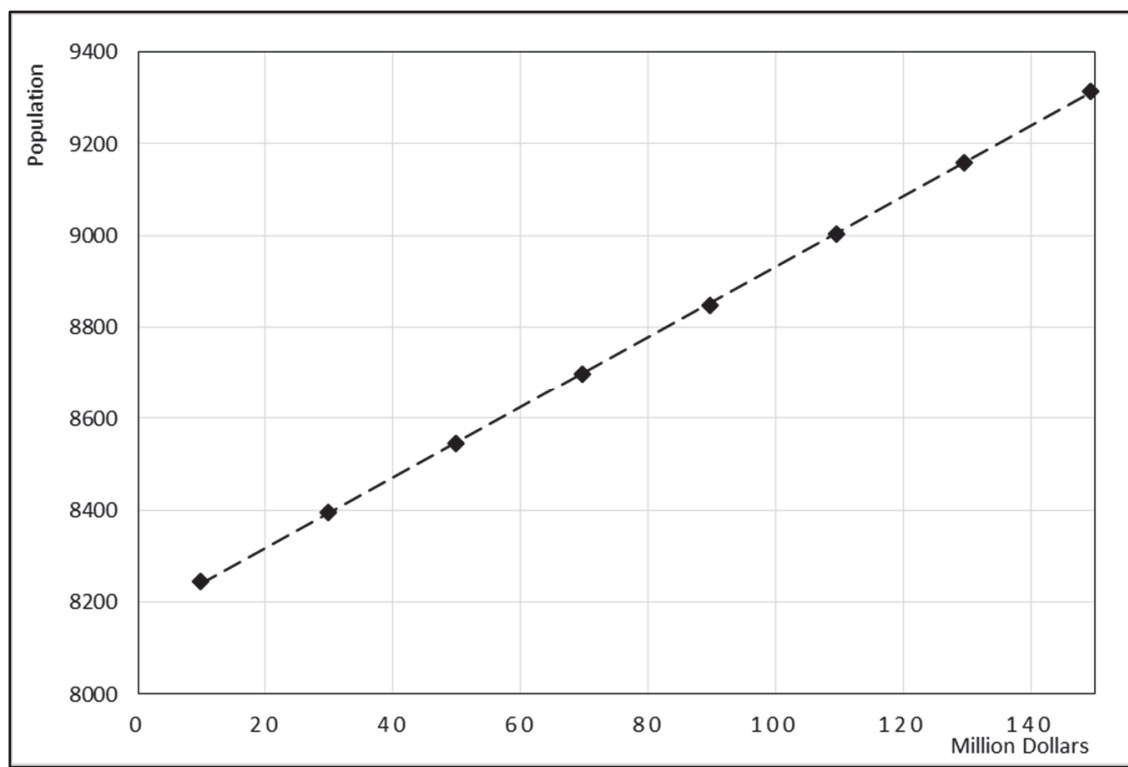

Figure 6.9 Average Population of the Low-Resilience Spatial Units Simulated with Different Mitigation Expenses

Another monotonous and almost liner relationship was found between the mitigation expenses and the final average population. The higher the mitigation expenses, the higher the final average population. These tests are under the hazards scenario with $\theta$ set to 1 , which means the yearly damages in the simulation equals the historical records. In order to find out what the average projected population will be under different combinations of hazards scenarios ( $\theta$ values) and mitigation expenses, a multilinear regression inspired by the two linear and monotonous relationships found above was built with the average projected population of the low-resilience spatial units as dependent variable, and $\theta$ and mitigation expenditures as 
independent variables (predictors) using the data points from Figure 6.8 and Figure 6.9, and the results are shown in Table 6.1.

Table 6.1 Multi-Linear Regression Results for the Average Population of the Low-Resilience Spatial Units (on $\theta$ and Mitigation Expenses)

\begin{tabular}{|l|l|l|l|l|}
\hline \multicolumn{5}{|c|}{ Estimated Coefficients } \\
\hline & Estimate & SE & tStat & pValue \\
\hline Intercept & 10124 & 64.954 & 155.87 & $1.1751 \mathrm{e}-22$ \\
\hline$\theta$ & -1791.2 & 53.739 & -33.331 & $5.6172 \mathrm{e}-14$ \\
\hline Mitigation Expense & 6.0834 & 0.5099 & 11.93 & $2.2405 \mathrm{e}-08$ \\
\hline Number of observations: 16, Error degrees of freedom: 13 & & \\
Root Mean Squared Error: 105 \\
R-squared: 0.99, Adjusted R-Squared 0.988 \\
F-statistic vs. constant model: 644, p-value = 1e-13
\end{tabular}

Table 6.1 shows that the data points fit the multi-linear regression model quite well with an adjusted R-squared value nearly equaled one. This linear regression model offers the foundation to evaluate the sustainability of the low-resilience spatial units. For example, if the sustainability criterion is to maintain an average population of 8000 people (around $63 \%$ of the average of the initial populations of the low-resilience spatial units) from 2010 to 2050 and the mitigation budget is 150 million every 10 year, then the sustainability goal, the mitigation budget, and the average simulated population can all be represented by a 3-dimensional plane, respectively, as shown in Figure 6.10.

In Figure 6.10, the plane of the sustainability goal and the plane of the simulated population separate the whole coordinate system into three spaces: the sustainable space, the mitigatable space, and the tipping space. In the sustainable space, the projected population is always higher than the sustainability goal, which means even without any mitigation efforts and planning, the low-resilience spatial units are still sustainable. In the mitigatable space, the projected population can either be higher than the sustainability goal (the upper triangular cube), or be lower than the sustainability goal (the lower triangular cube). In this space, the sustainability of these spatial units is determined by the mitigation cost line. If the actual cost is above this line, these spatial units are sustainable (the upper space), whereas if the actual cost is below this line (the lower space), these spatial units are not sustainable. In the tipping 
space, the projected population is always below the sustainability goal, which means under the given mitigation budget, these spatial units will never be sustainable

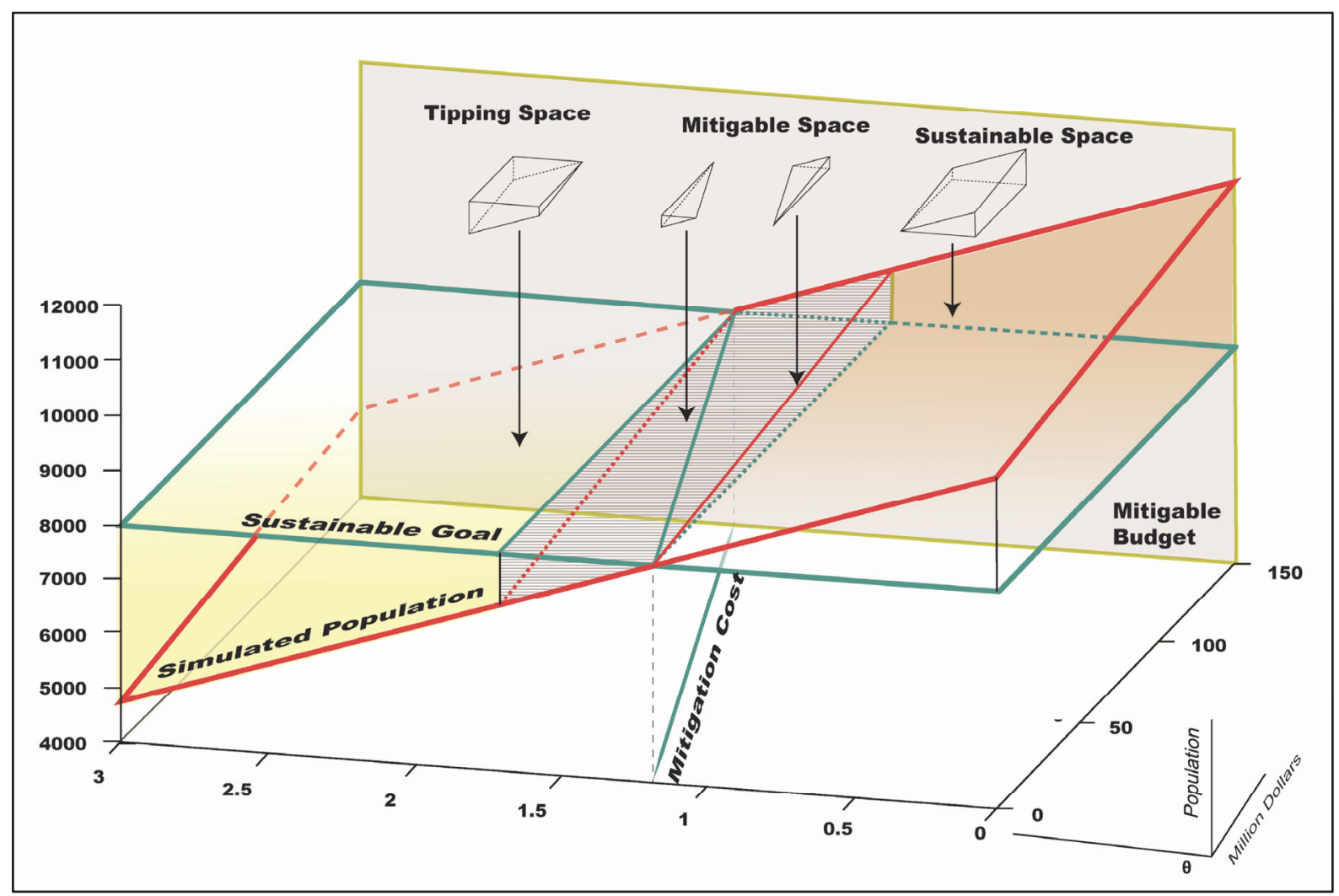

Figure 6.10 Sustainability Analysis of the Low-Resilience Spatial Units with Mitigation Budget Set to 150 Million Dollars per 10 Year and Sustainability Goal Set to 8000 People

\subsubsection{Sustainability of the High-Resilience Areas}

The spatial units in the usurper and the resistant groups are identified as high resilience areas. For these areas, population growths with different rates have been observed. Several simulations were run in the same way to test the response of the final average population of these spatial units to different $\theta$ values (Figure 6.11) and different mitigation expenditures (Figure 6.12). 


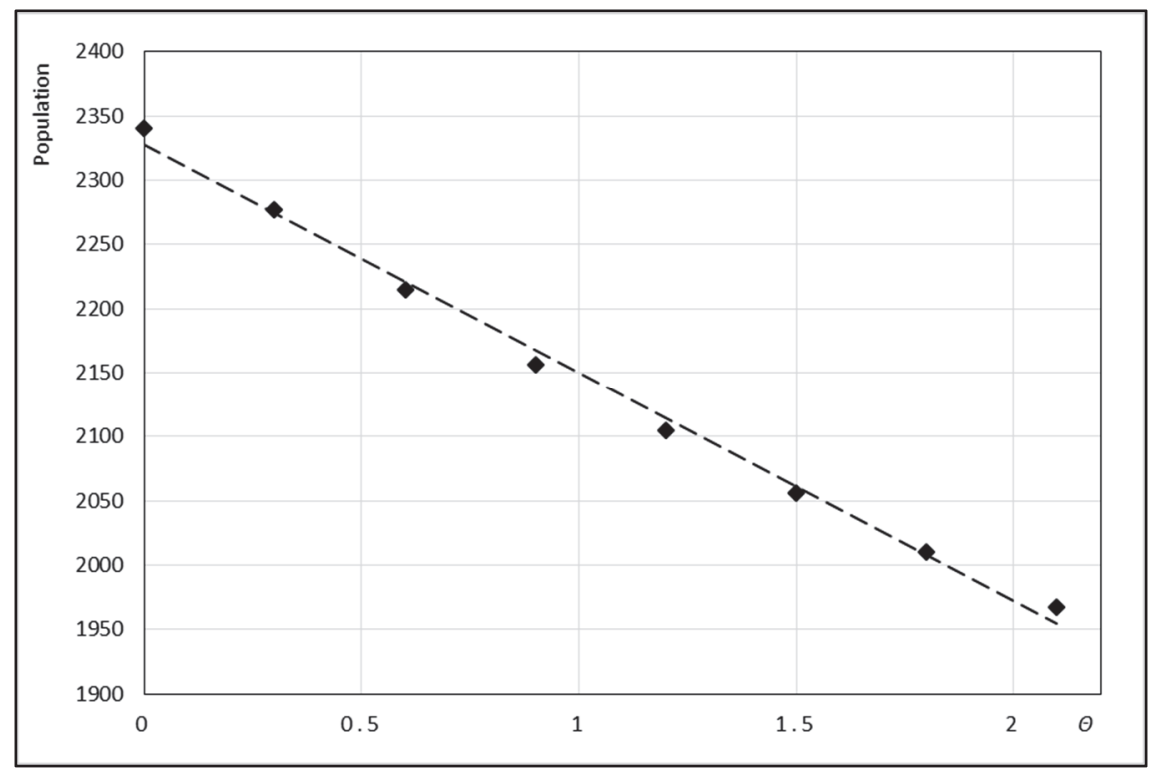

Figure 6.11 Average Population of the High-Resilience Spatial Units Simulated with Different $\theta$ Values

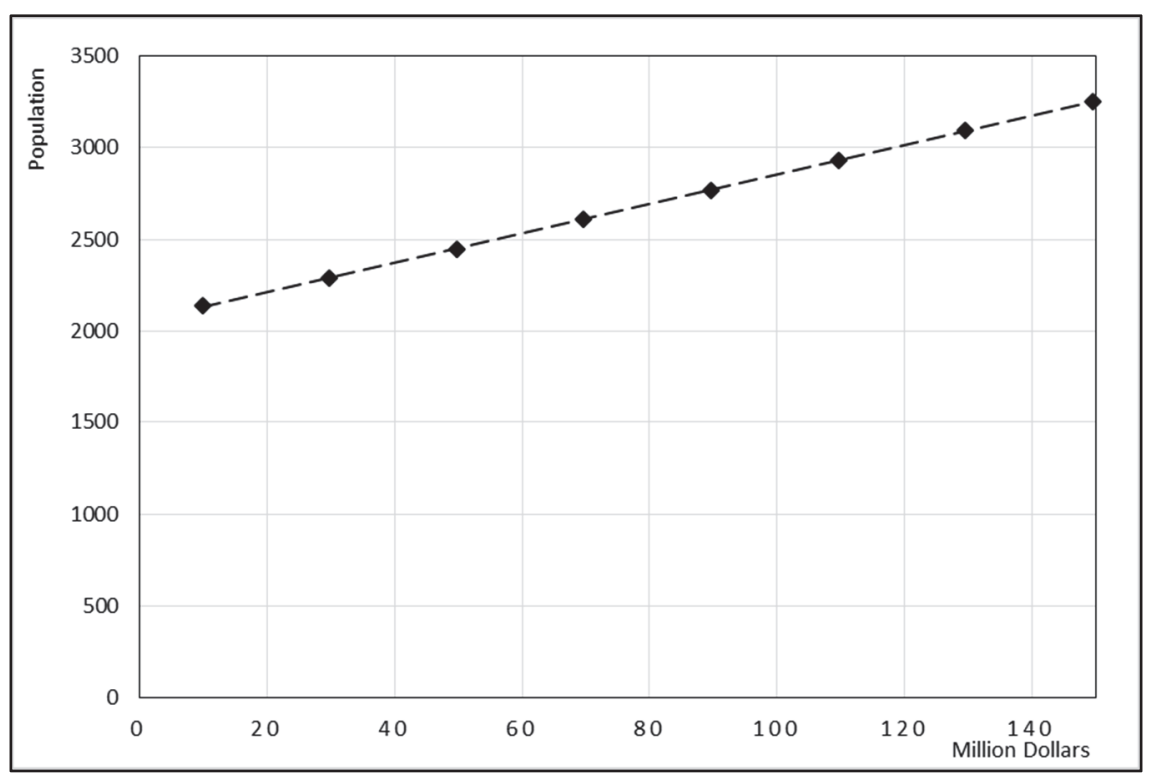

Figure 6.12 Average Population of the High-Resilience Spatial Units Simulated with Different Mitigation Expenses

A multi-linear regression model was also built by using the simulation results from the two figures above to find out what is the average projected population under different combinations of hazards scenarios ( $\theta$ values) and mitigation expenses, and the results are shown in Table 6.2. 
Table 6.2 Multi-Linear Regression Results for the Average Population of the High Resilience Spatial Units (on $\theta$ and Mitigation Expenses)

\begin{tabular}{|c|c|c|c|c|}
\hline \multicolumn{5}{|c|}{ Estimated Coefficients } \\
\hline & Estimate & $\mathrm{SE}$ & tStat & pValue \\
\hline Intercept & 2306.1 & 21.54 & 107.06 & $1.5458 \mathrm{e}-20$ \\
\hline$\theta$ & -175.52 & 17.821 & -9.8489 & $2.1462 \mathrm{e}-07$ \\
\hline Mitigation Expense & 7.2788 & 0.1691 & 43.045 & $2.0788 \mathrm{e}-15$ \\
\hline \multicolumn{5}{|c|}{$\begin{array}{l}\text { Number of observations: } 16 \text {, Error degrees of freedom: } 13 \\
\text { Root Mean Squared Error: } 34.7 \\
\text { R-squared: } 0.993 \text {, Adjusted R-Squared } 0.992 \\
\text { F-statistic vs. constant model: } 993, \text { p-value }=6.08 \mathrm{e}-15\end{array}$} \\
\hline
\end{tabular}

Table 6.2 shows that the multi-linear model is well fitted with an adjusted R-squared very closed to one. A sustainability analysis of the high-resilience spatial units was conducted in the same manner as the one conducted for the low-resilience spatial units. A sustainability goal was set to maintain a population of 1,500 people (approximately the average of the initial population of these high-resilience spatial units in 2010). The mitigation budget was set to 150 million dollars per decade (the same budget as the low-resilience spatial units). Figure 6.13 was created by plotting the simulated population plane, the mitigation budget plane, and the sustainability goal plane together.

From Figure 6.13, there is only one sustainable space in the three dimension coordinate system, in which the projected average population is always higher than the sustainability goal. Even with the mitigation expenses set to 0 and the $\theta$ value set to 3 , the projected average population is still above the sustainability goal, which means that for these high-resilience spatial units, even without any mitigation expenditures and with three times the historical hazard damages, they will still get population growth. 


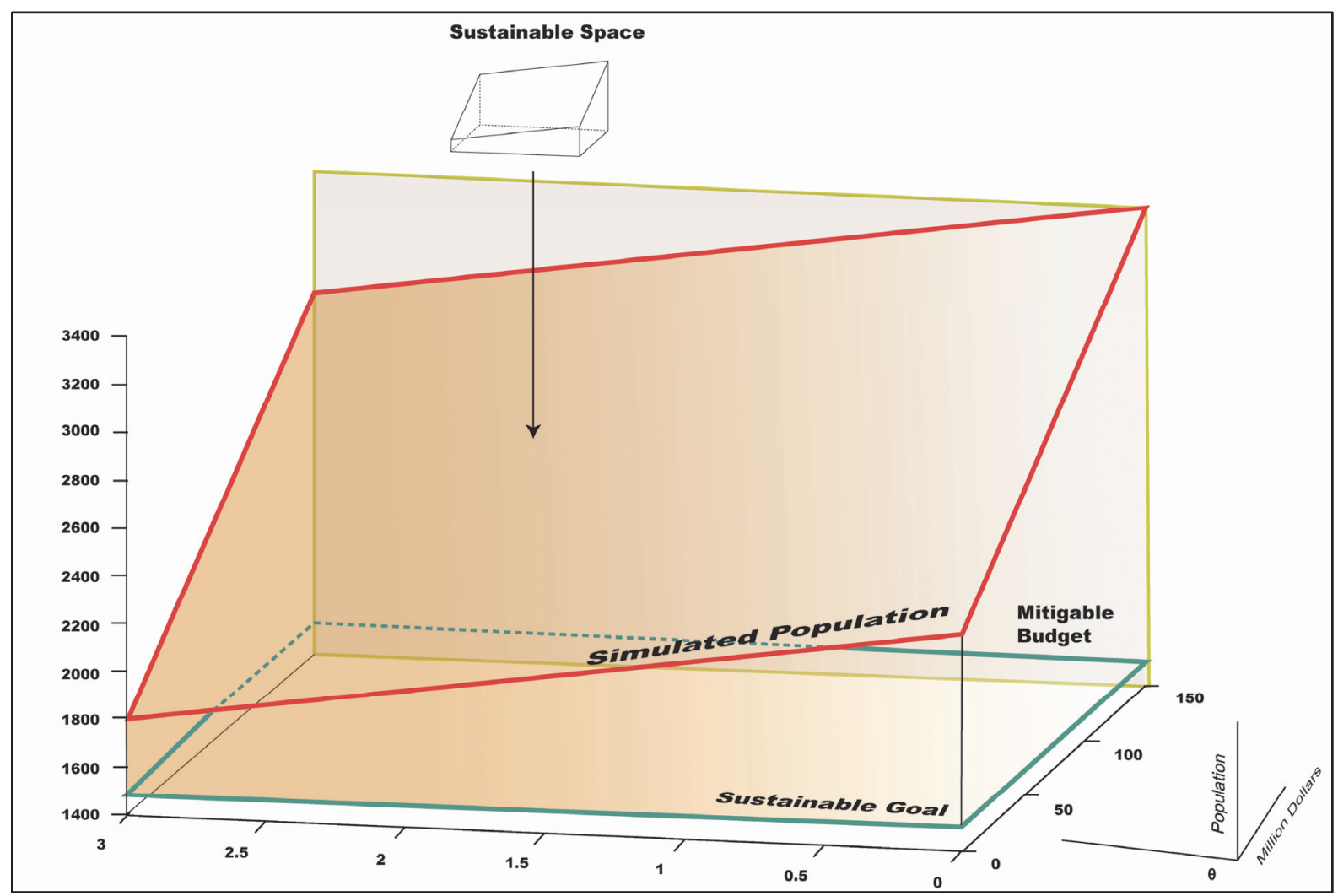

Figure 6.13 Sustainability Analysis of the High Resilience Spatial Units with Mitigation Budget set to 150 Million Dollars and Sustainability Goal set to 1500 People 


\subsection{References}

Bação, F., Lobo, V., Painho, M. (2005). Self-organizing Maps as substitutes for K-means clustering. Lecture Notes in Computer Science Computational Science ICCS 2005, 476-483.

Bodt, E. D., Verleysen, M., Cottrell, M. (1997). Kohonen maps versus vector quantization for data analysis. ESANN, Bruges.

Fisher, R. A. (1936). The use of multiple measurements in taxonomic problems. Annals of Eugenics 7(2): 179-188.

Lam, N., Reams, M., Li, K., Li, C., Mata, L. (2015a). Measuring community resilience to coastal hazards along the northern Gulf of Mexico. Natural Hazards Review.

Lam, N.S.N., Qiang, Y., Arenas, H, Brito P., Liu, K.B. (2015b). Mapping and assessing coastal resilience in the Caribbean region. Cartography and Geographic Information Science 42(4): 315-322.

Li, K. (2011). Temporal changes of coastal community resilience in the Gulf of Mexico Region. Master's Thesis. Baton Rouge, Louisiana: Louisiana State University.

Li, K., Lam, N., Qiang, Y., Zou, L., Cai, H. (2015). A cyberinfrastructure for community resilience assessment and visualization. Cartography and Geographic Information Science 34-39.

Möller-Levet, C.S., Klawonn, F., Cho, K.H., Wolkenhauer, O. (2003). Fuzzy clustering of short time series and unevenly distributed sampling points, Proceedings of the 5th International Symposium on Intelligent Data Analysis, Berlin, Germany.

Sejnowski, T. J., Gorman, P. (1988). Learned classification of sonar targets using a massively parallel network. IEEE Transactions on Acoustics, Speech, and Signal Processing 36(7): $1135-1140$. 


\section{CHAPTER 7 RELATIVE LAND PRICE ESTIMATION}

A measurement called the Relative Land Price is introduced in this Chapter from evaluating the relationship between the utility and the population of a spatial unit. The Relative Land Price is used to indicate the relative surplus value of a spatial unit in this study area due to its population and its utility.

\subsection{Utility and Mitigation Expenses}

The Utility as defined in Chapter 4 is a linear combination of the standardized values of selected social and economic variables, and the higher the utility value, the higher the tendency of population growth. In addition to the high hazard damages, the low-resilience spatial units also have lower than average utility values. In this section, the objective is to test whether increasing the utility of these spatial units can offer a substitute option for increasing the possibility to achieve the sustainability goal or not. A relationship was found between the increase of utility and the increase of mitigation expenses, based on which the Relative Land Price is defined in the following section.

\subsubsection{Low-Resilience Spatial Units}

Eight simulations ware tested with the utilities of the low-resilience spatial units increased, and the results are shown in Figure 7.1. As Figure 7.1 shows, the utility has the same ability as the mitigation expenses to increase the projected population, and is also positively and linearly correlated with the final average projected population. A multi-linear regression model was built with the average projected population as dependent variable and the mitigation expenditure and the increase of utility as independent variables, using the data points from Figure 6.9 and Figure 7.1 as sample points, and the results are shown in Table 7.1. 


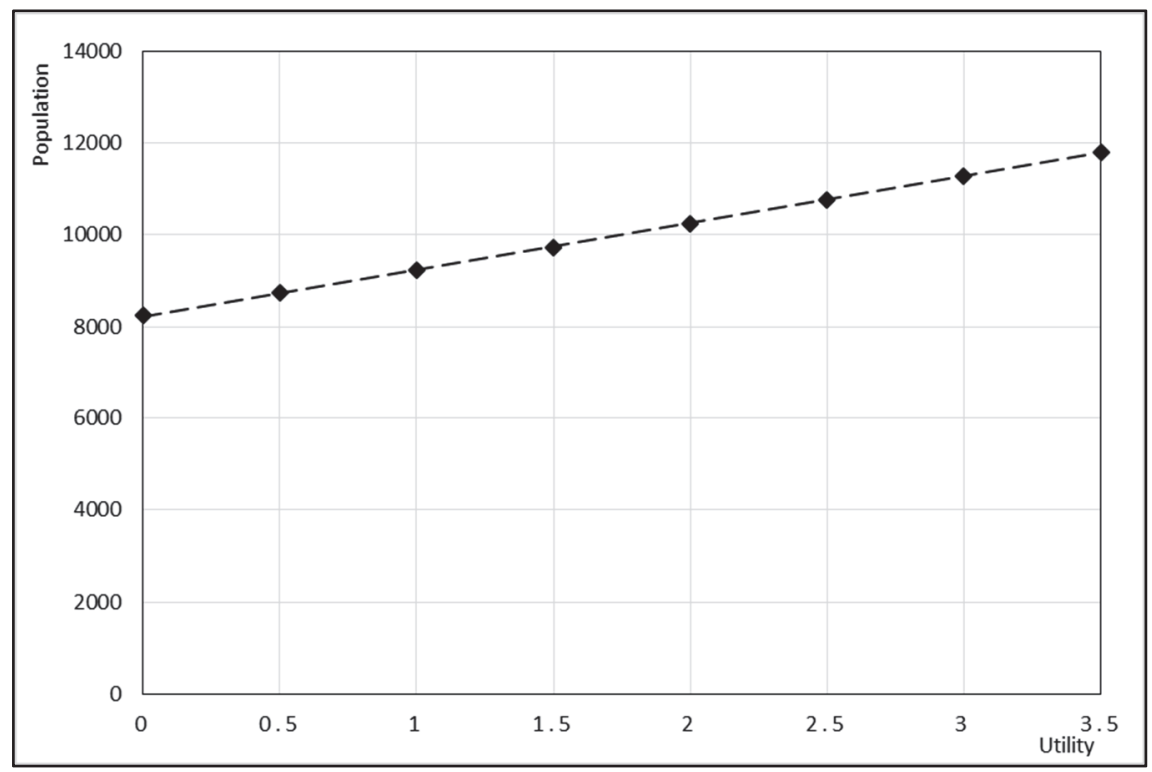

Figure 7.1 Average Population of the Low-Resilience Spatial Units Simulated with Different Utility Increases

Table 7.1 Multi-Linear Regression Results for the Average Population of the Low Resilience Spatial Units on Utility and Mitigation Expenses

\begin{tabular}{|c|l|l|l|l|}
\hline \multicolumn{5}{|c|}{ Estimated Coefficients } \\
\hline & Estimate & SE & tStat & pValue \\
\hline Intercept & 8194 & 9.0526 & 905.16 & $1.3798 \mathrm{e}-32$ \\
\hline Utility & 1030.7 & 4.8339 & 213.22 & $2.0032 \mathrm{e}-24$ \\
\hline Mitigation Expense & 7.3951 & 0.11237 & 65.807 & $8.5465 \mathrm{e}-18$ \\
\hline $\begin{array}{l}\text { Number of observations: 16, Error degrees of freedom: } 13 \\
\text { Root Mean Squared Error: 18.9 } \\
\text { R-squared: 1.0, Adjusted R-Squared 1.0 } \\
\text { F-statistic vs. constant model: 2.5e+04, p-value }=\text { 4.93e-24 }\end{array}$ \\
\hline
\end{tabular}

The multi-linear regression function with the coefficients as shown in Table 7.1, fits the data points perfectly with an adjusted R-squared value equaled 1 . This function is represented by a plane in a 3-dimension coordinate system, as shown in Figure 7.2. In Figure 7.2, the two horizontal planes with border marked by black dash lines in the coordinate system are the planes with the same population. They intersect the simulated population plane at the two lines marked by red solid lines. Points A, B and C are three sample points taken from the two lines. The plane that point $\mathrm{B}$ and point $\mathrm{C}$ belonged to has a population of 10,000 people, and the plane that point A belonged to has a population of 9,000 people. In order to increase the population by 1,000 people from point $\mathrm{A}$, either that an increase of the utility value is needed (point $\mathrm{A}$ becomes point $\mathrm{C}$ ), or that an increase of the mitigation expenses is needed (the 
A becomes point B). Thus, for the purpose of increasing the population, the increase of the utility values between point A and point B is the "same" as the increase of the mitigation expenses between point $\mathrm{A}$ and point $\mathrm{C}$.

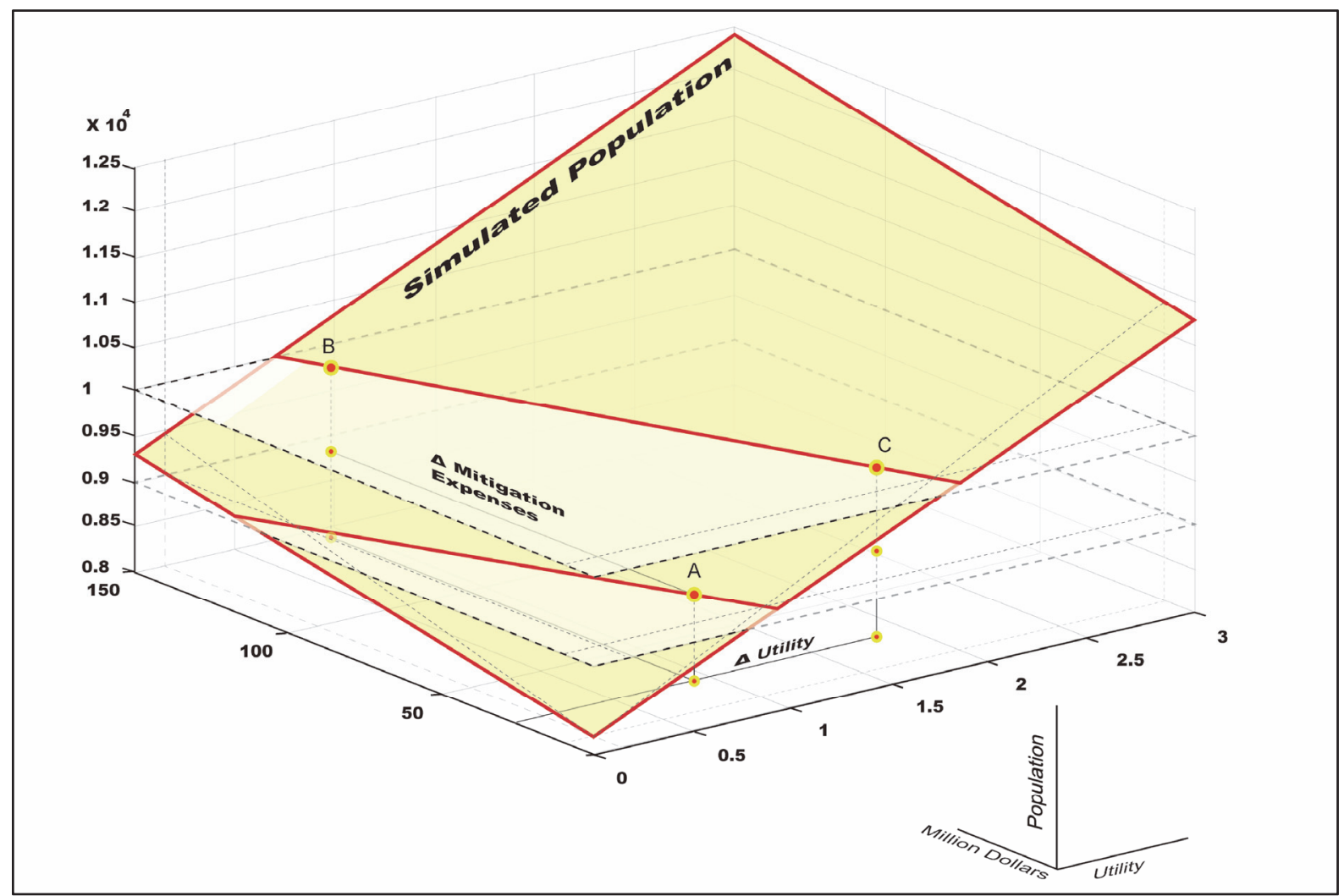

Figure 7.2 Average Population of the Low-Resilience Spatial Units for Different Utility and Mitigation Expenses

\subsubsection{High-Resilience Spatial Units}

Similarly, another set of eight simulations were conducted for the high-resilience spatial units, and the results of the average projected populations with increasing utilities are shown in Figure 7.3. 


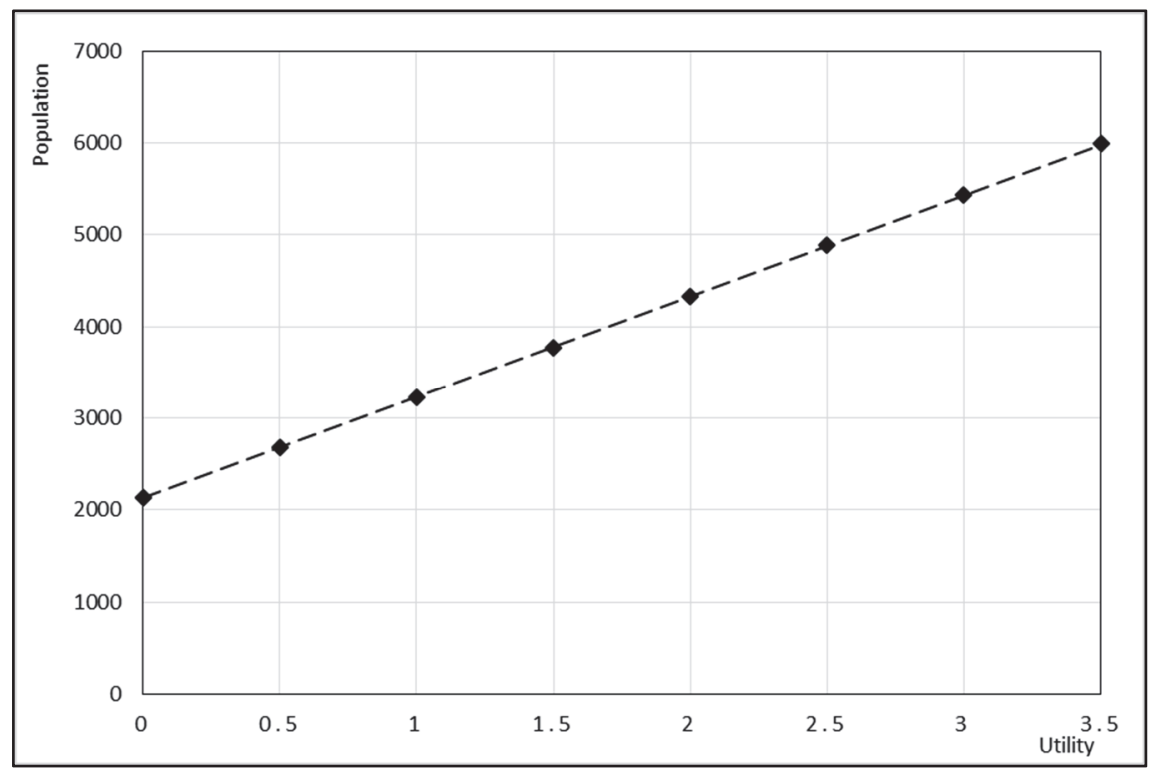

Figure 7.3 Average Population of the High-Resilience Spatial Units Simulated with Different Utility Increases

A multi-linear regression model was built by using the simulation results from Figure 6.12 and Figure 7.3, in order to find out what the average projected population will be under different combinations of utility values and mitigation expenses for the high-resilience spatial units, and the results are shown in Table 7.2.

Table 7.2 Multi-Linear Regression Results for the Average Population of the High-Resilience Spatial Units on Utility and Mitigation Expenses

\begin{tabular}{|c|c|c|c|c|}
\hline \multicolumn{5}{|c|}{ Estimated Coefficients } \\
\hline & Estimate & $\mathrm{SE}$ & tStat & pValue \\
\hline Intercept & 2093.6 & 10.514 & 199.11 & $4.8767 \mathrm{e}-24$ \\
\hline Utility & 1115.2 & 5.6145 & 198.62 & $5.0352 \mathrm{e}-24$ \\
\hline Mitigation Expense & 7.6281 & 0.13052 & 58.443 & $3.9782 \mathrm{e}-17$ \\
\hline \multicolumn{5}{|c|}{$\begin{array}{l}\text { Number of observations: 16, Error degrees of freedom: } 13 \\
\text { Root Mean Squared Error: } 22 \\
\text { R-squared: } 1.0 \text {, Adjusted R-Squared } 1.0 \\
\text { F-statistic vs. constant model: } 2.19 \mathrm{e}+04, \mathrm{p} \text {-value }=1.16 \mathrm{e}-23\end{array}$} \\
\hline
\end{tabular}

The multi-linear equation built from the above table is plotted into a 3-dimensin coordinate system as shown in Figure 7.4. 


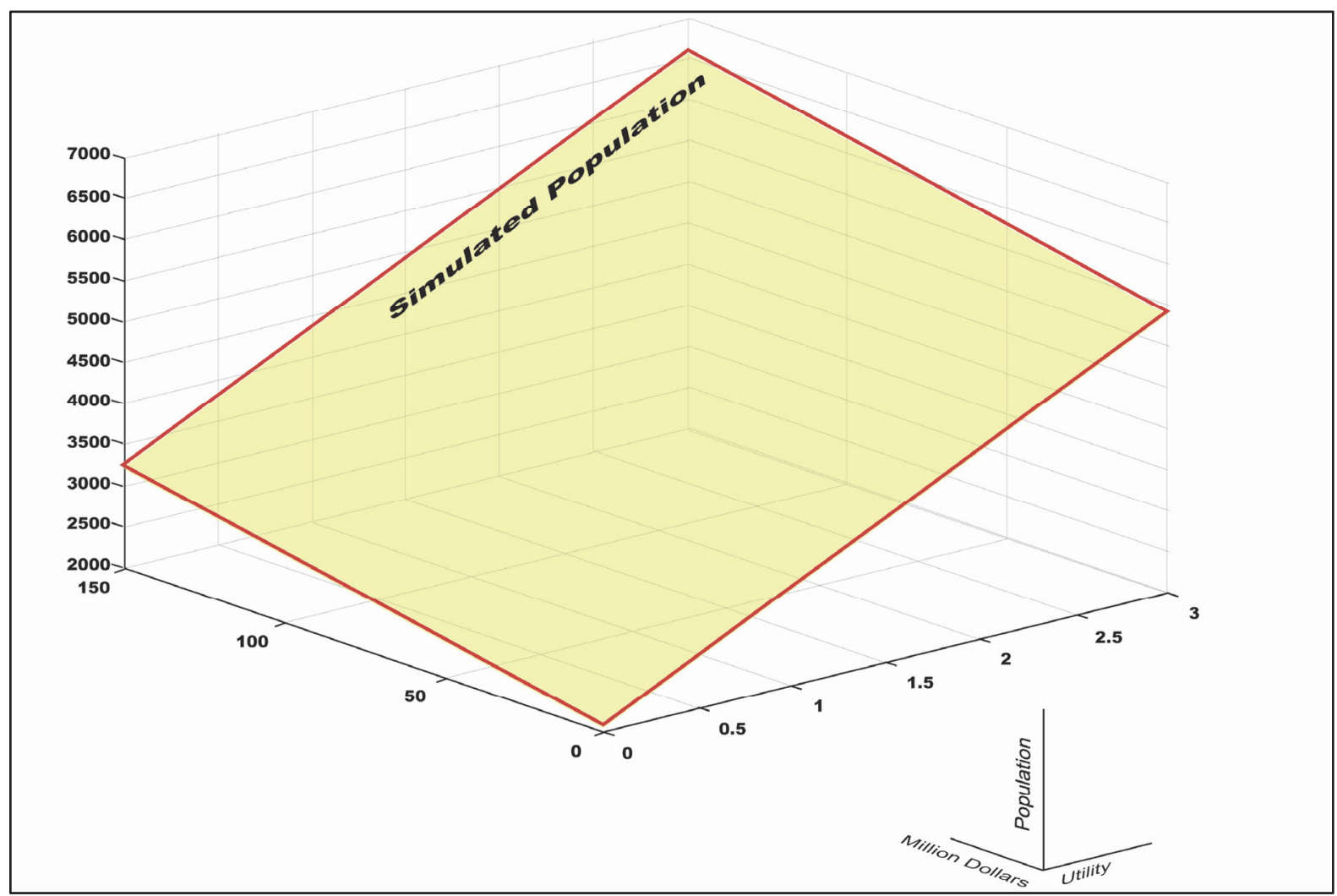

Figure 7.4 Average Population of the High-Resilience Spatial Units for Different Utility and Mitigation Expenses

\subsection{Relative Land Price}

The relative land price is defined as the relative value of a spatial unit due to its population and its utility among a given set of spatial units. A spatial unit with zero population and zero utility is assumed to have a relative land price of zero dollars. It should be noted that the relative land price defined here is very different from the economic definition of land price, in which the market value of a certain land area is decomposed into structures and land components, and land price is the estimated price of the land component (Davisa and Heathcoteb, 2007). In contrast with the "economic land price", which focuses on the surplus value of a land area due to its human structures, the relative land price defined here focuses on the surplus value of the a land area due to its population and the correlated utility. From Section 7.1, a relationship was found as represented by the following equation:

$$
\Delta \text { Population }=a \Delta U \text { tility }+b \Delta \text { Expenses }
$$


where $a$ and $b$ are the coefficients calculated by the multi-linear regression. From Equation 7.1, the expenses $e$ to change a spatial unit with zero population and zero utility into a spatial unit with a population of $p$ and a utility of $u$ can be calculated according to Equation 7.2:

$$
e=\frac{p-a u}{b}
$$

For both the low-resilience and the high-resilience spatial units, their Relative Land Price planes with the coefficients estimated by the multi-linear regressions are shown in Figure 7.5.

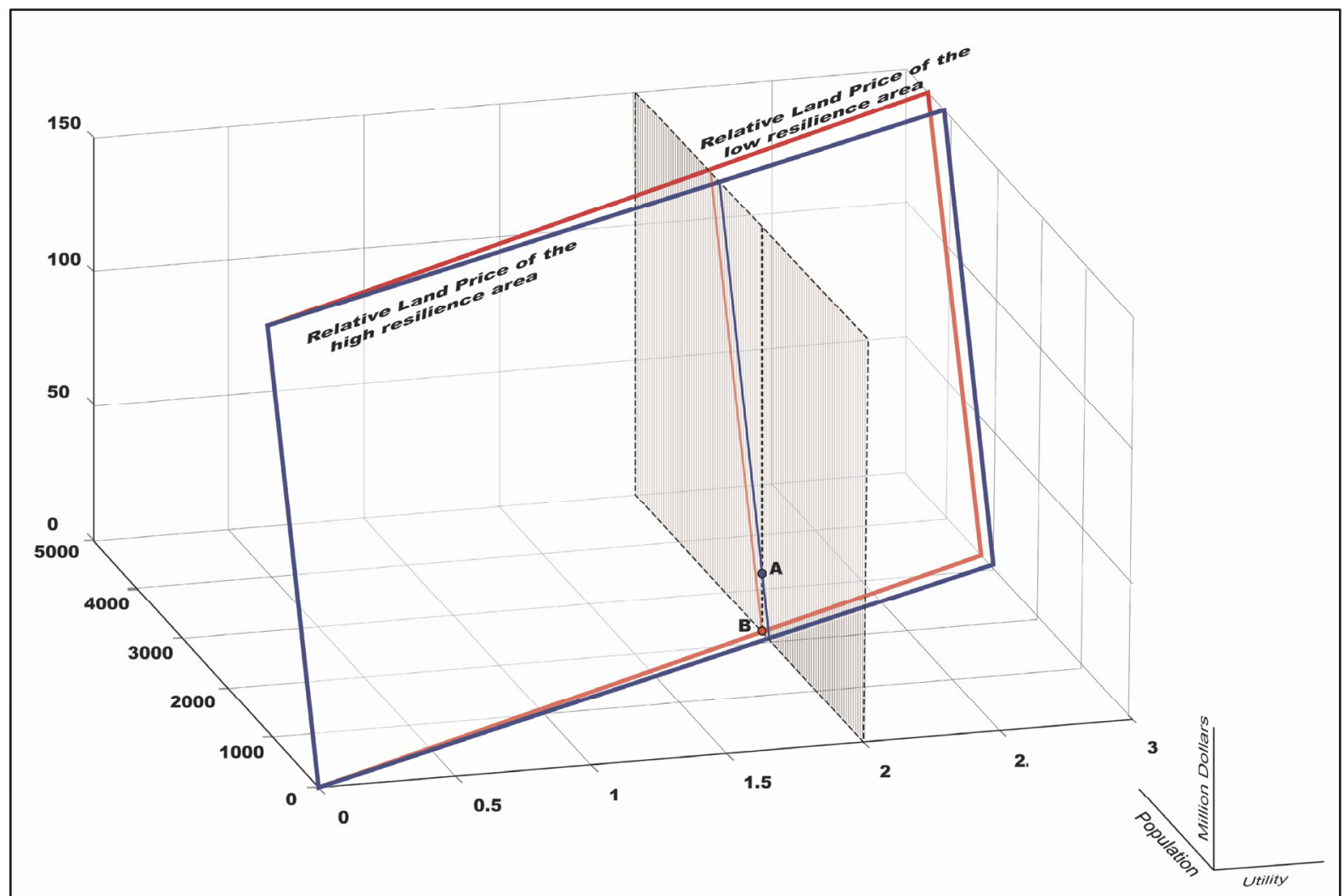

Figure 7.5 The Relative Land Price of the High Resilience Spatial Units and the Relative Land Price of the Low Resilience Spatial Units

In Figure 7.5, the plane with the borders marked by blue lines represents the function for calculating the Relative Land Price for the high-resilience spatial units (short for highresilience plane), and the plane with the borders marked by red lines represents the function 
for calculating the Relative Land Price for the low-resilience spatial units (short for lowresilience plane).

First, for a fixed value of utility (the light gray plane), the marginal relative land prices over population represented by the slopes of the intersection lines between the light grey plane and each of the resilience planes (for both the high and the low resilience spatial units), are almost the same between the low-resilience and the high-resilience groups. In other words, for a spatial unit with a fixed utility, its marginal Relative Land Price over population does not quite related with its resilience capacity. This means that the economically efficiency to increase the population in the low-resilience areas is about the same as in the high-resilience areas given a fixed utility. In other words, a dollar increase of the Relative Land Price will have similar impacts on population growth for the low-resilience and the high-resilience areas in this given condition.

Second, for a fixed value of utility and a fixed value of population, the Relative Land Price on the high resilience plane (point A) is higher than the Relative Land Price on the low resilience plane (point B). This means that for two given spatial units with the same utility and population, one of which is in the low-resilience group, and the other of which is in the highresilience group, the Relative Land Price of the one in the high-resilience group will be higher. In other words, the surplus value of a spatial unit due to its population and utility is higher in the high-resilience group. It also means that it may be more economically efficient to achieve the same population and utility goal in the low-resilience areas than in the high-resilience areas.

According to the definition of the Relative Land Price, the calculation of it depends on the coefficients in the multi-linear regression function of expenses, utility, and population, thus the value of it is only comparable within the spatial units from which this function is derived. 
In order to evaluate the Relative Land Price of the whole metropolitan area in this study, the function for the population, the utility, and the expenses need to be re-derived. Eight simulations were run to test the relationship between the final average population and the amount of mitigation expenses for all the urban spatial units, and the results are shown in Figure 7.6.

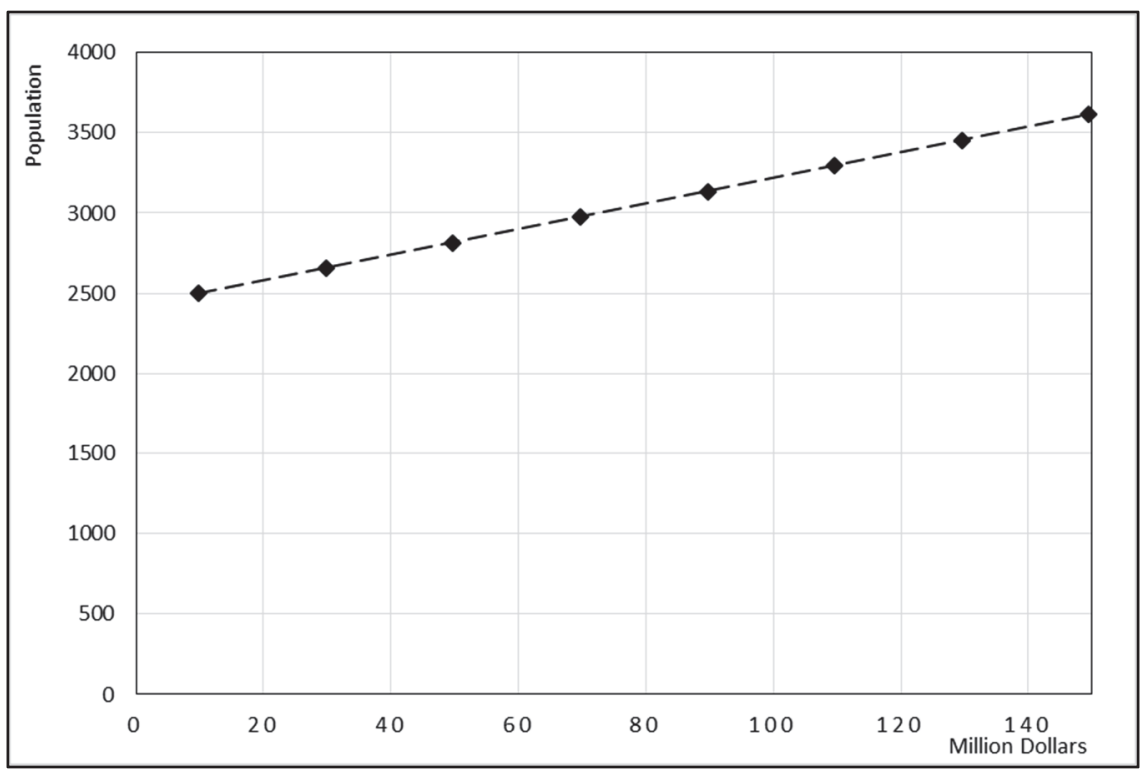

Figure 7.6 Average Population of all the Spatial Units in the City Areas of the Study Area Simulated with Different Mitigation Expenses

As Figure 7.6 shows, the simulated population linearly, positively correlates with the mitigation expenses. The relationship between the simulated population and the value of the utility was also tested for all the urban spatial units and the results are shown in Figure 7.7. 


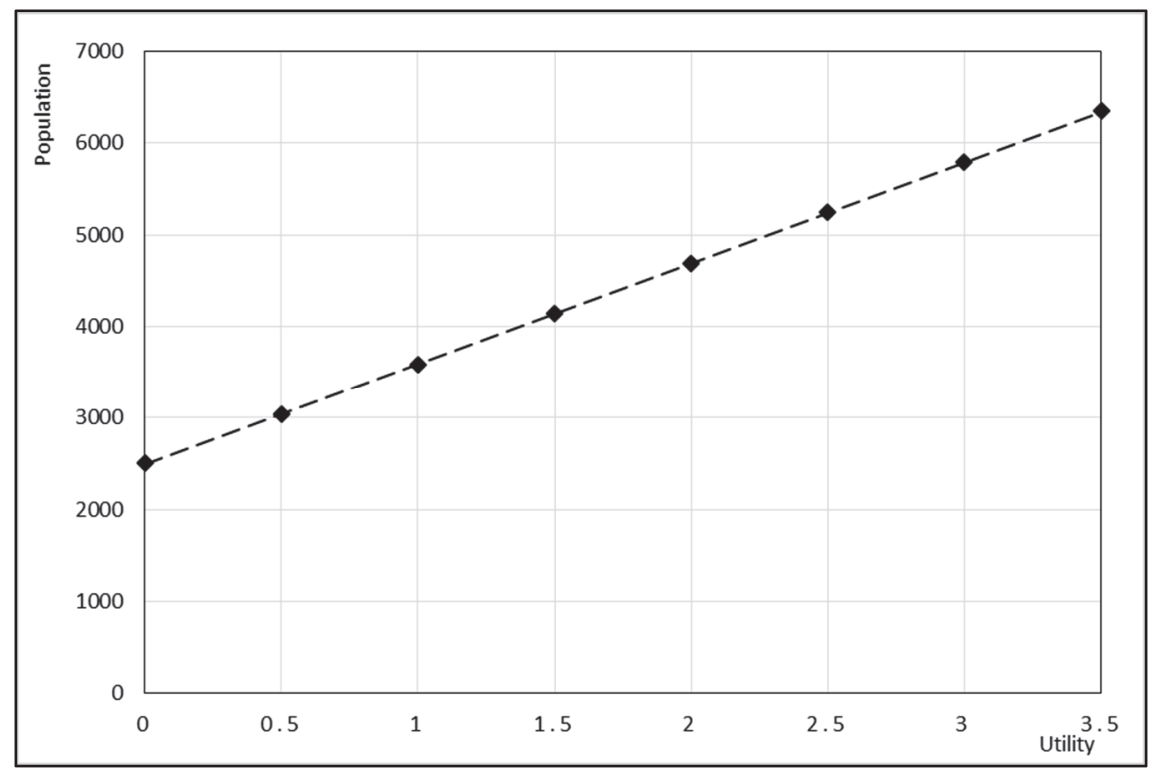

Figure 7.7 Average Population of all the Spatial Units in the City Areas of the Study Area Simulated with Different Utility Increases

From Figure 7.7, the simulated population was also found to have a positively and linearly correlation with the utility. After running the multi-linear regression on the data points in Figure 7.6 and Figure 7.7, the results are shown in Table 7.3.

Table 7.3 Multi-Linear Regression Results for the Average Population of all the Spatial Units in City Areas of the Study Area on Utility and Mitigation Expenses

\begin{tabular}{|c|c|c|c|c|}
\hline \multicolumn{5}{|c|}{ Estimated Coefficients } \\
\hline & Estimate & SE & tStat & pValue \\
\hline Intercept & 2459.3 & 10.38 & 236.93 & $5.0874 \mathrm{e}-25$ \\
\hline Mitigation Expenses & 7.6119 & 0.12885 & 59.077 & $3.4594 \mathrm{e}-17$ \\
\hline Utility & 1112.5 & 5.5424 & 200.72 & $4.3924 \mathrm{e}-24$ \\
\hline $\begin{array}{l}\text { Number of observations: 16, } \\
\text { Root Mean Squared Error: } 21 \\
\text { R-squared: 1.0, Adjusted R-S } \\
\text { F-statistic vs. constant model: }\end{array}$ & $\begin{array}{l}\text { grees of fr } \\
1.0 \\
04, \text { p-value }\end{array}$ & & & \\
\hline
\end{tabular}

The multi-linear regression function with the coefficients as shown in Table 7.3, fits the data points perfectly with an adjusted R-squared value equaled 1 . This function is represented by a plane in a 3 -dimensional coordinate system, as shown in Figure 7.8, to show the relative land price planes for both the high-resilience spatial units and the low-resilience spatial units together. 


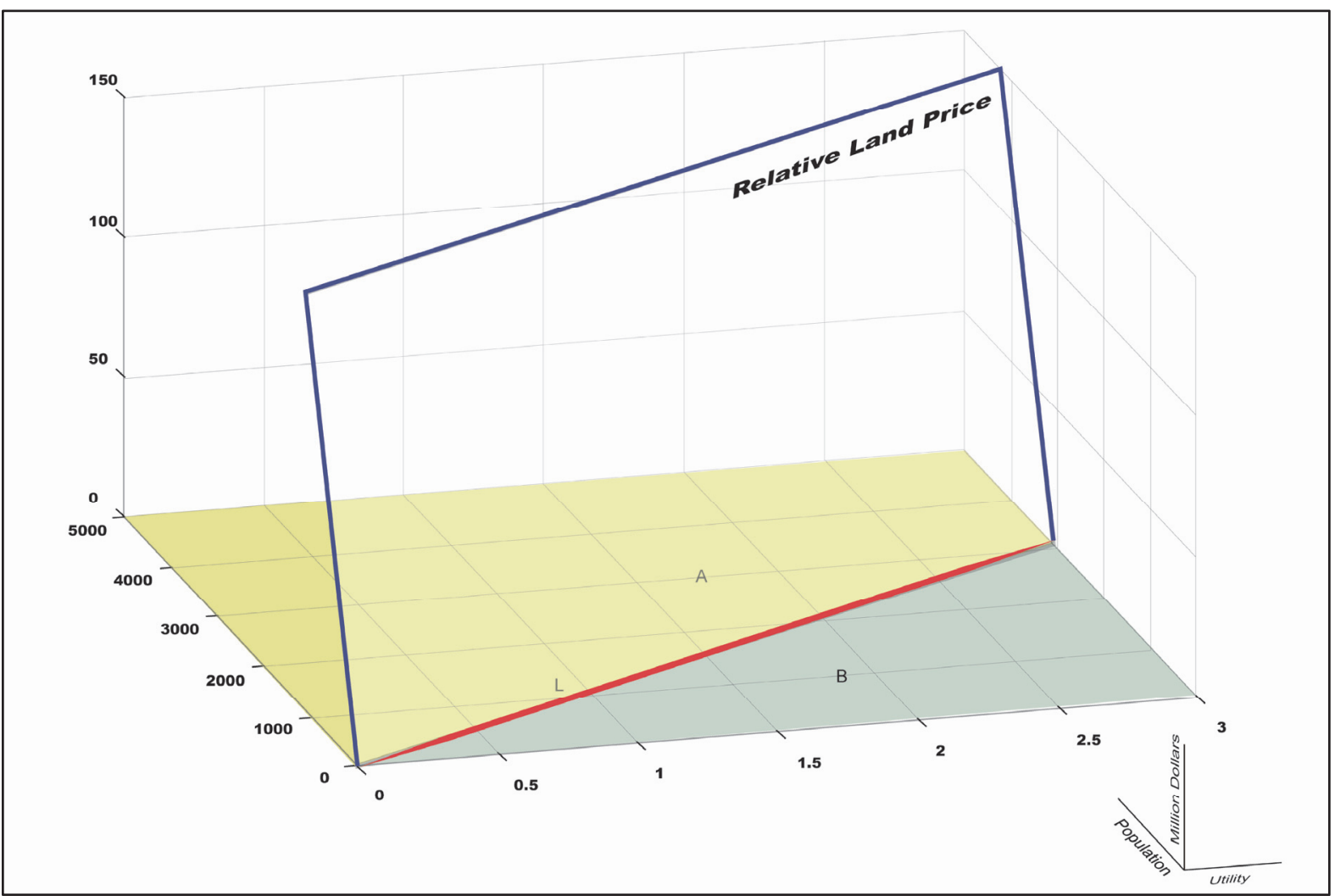

Figure 7.8 The Relative Land Price of all the Spatial Units in the City Areas of the Study Area

In Figure 7.8, the bottom horizontal plane is partitioned by the line $L$ into zone $A$ and zone $B$, and it is the plane where the Relative Land Price equals 0 . If a spatial unit has a combination of population and utility value on line $L$, it means that this spatial unit has a Land Price equaled 0, which means that this spatial unit does not have a surplus value due to its population and utility. If a spatial unit has a combination of population and utility value that falls into area $A$, it means that this spatial unit has a positive Relative Land Price and reflects positive surplus value. Similarly, if a spatial unit has a combination of the population and the utility value that falls into area $B$, it means that it have a negative Relative Land Price and reflects negative surplus value. 


\subsection{References}

Davis, M., Heathcote, J. (2007). The price and quantity of residential land in the United States. SSRN Electronic Journal SSRN Journal. 


\section{CHAPTER 8 COUPLED NATURAL AND HUMAN DYNAMICS OF THE "NORTH" AND THE "SOUTH"}

In this Chapter, the simulation results will be compared between the "north" and the "south" (Lam et al., 2012; Qiang and Lam, 2015), as well as between the city area of Baton Rouge (the major metropolitan area in the north) and the city area of New Orleans (the major metropolitan area in the south) (Figure 8.1). The analysis in this Chapter is based on the results from the simulations using the real historical data as the scenario (i.e. with $\theta$ value equaled one, without changes in utility values and without mitigation expenses).

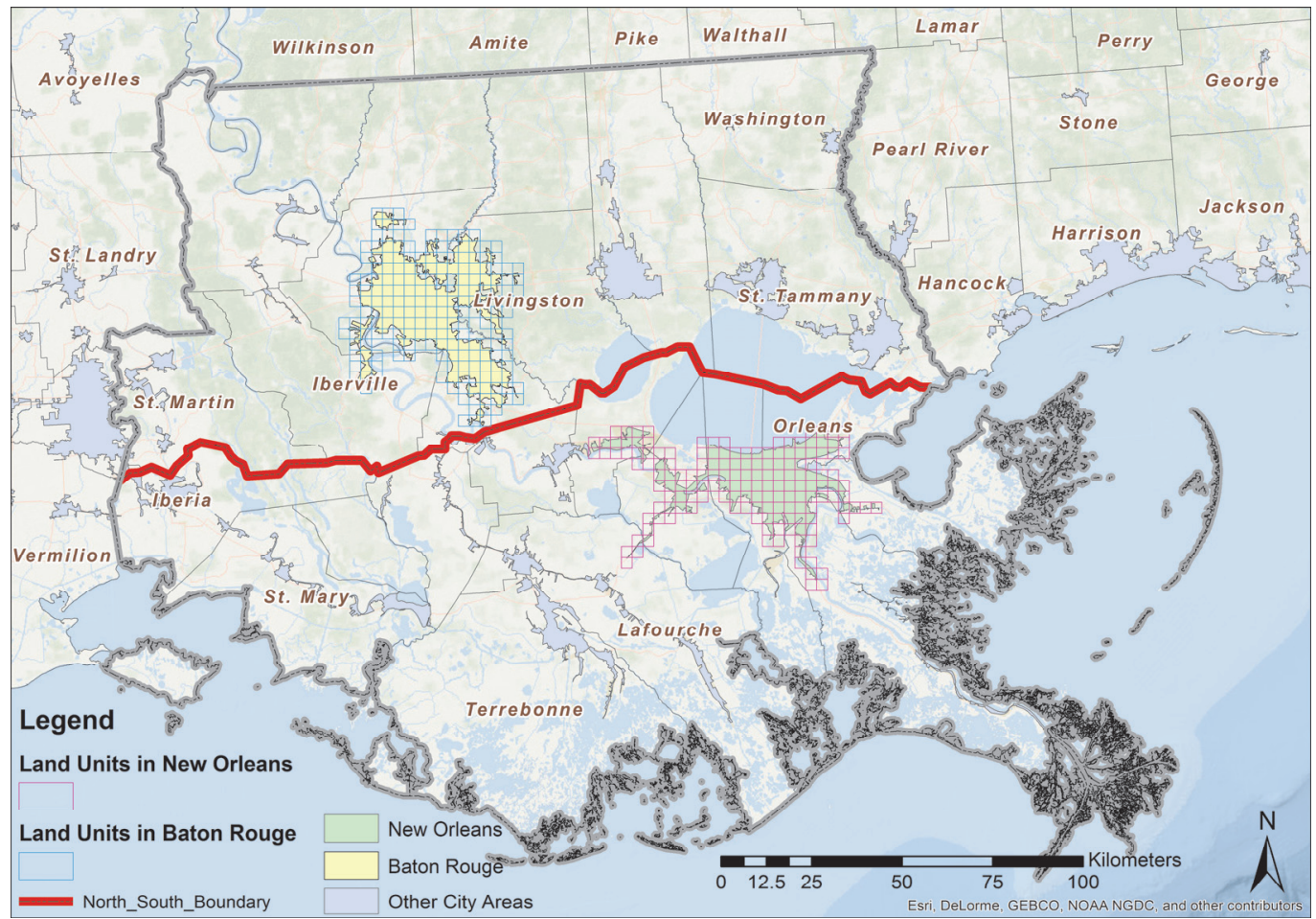

Figure 8.1 The City Areas in the Study Area with the "North" and "South" Boundary

\subsection{Comparison of the Populations}

The average simulated population time series of the spatial units belonged to the city areas in the "north" and the "south" were tabulated for a short time period (from 2010 to 2050) and a long time period (from 2010 to 2210). Only the populations of the spatial units belonged to the city areas (Figure 8.1) were analyzed and compared (totally 901 spatial units), due to the reason that the other "rural" spatial units have very low populations. For the short time-period 
simulation, the concern is the sustainability of the "north" and the "south" in terms of population. In contrast, for the long time-period simulation, the concern is the existence of the break points (or turning points). In other words, if the average populations of the "north" and the "south" region will change from decreasing to increasing or vice versa. The simulation results are shown in Figure 8.2 and 8.3.

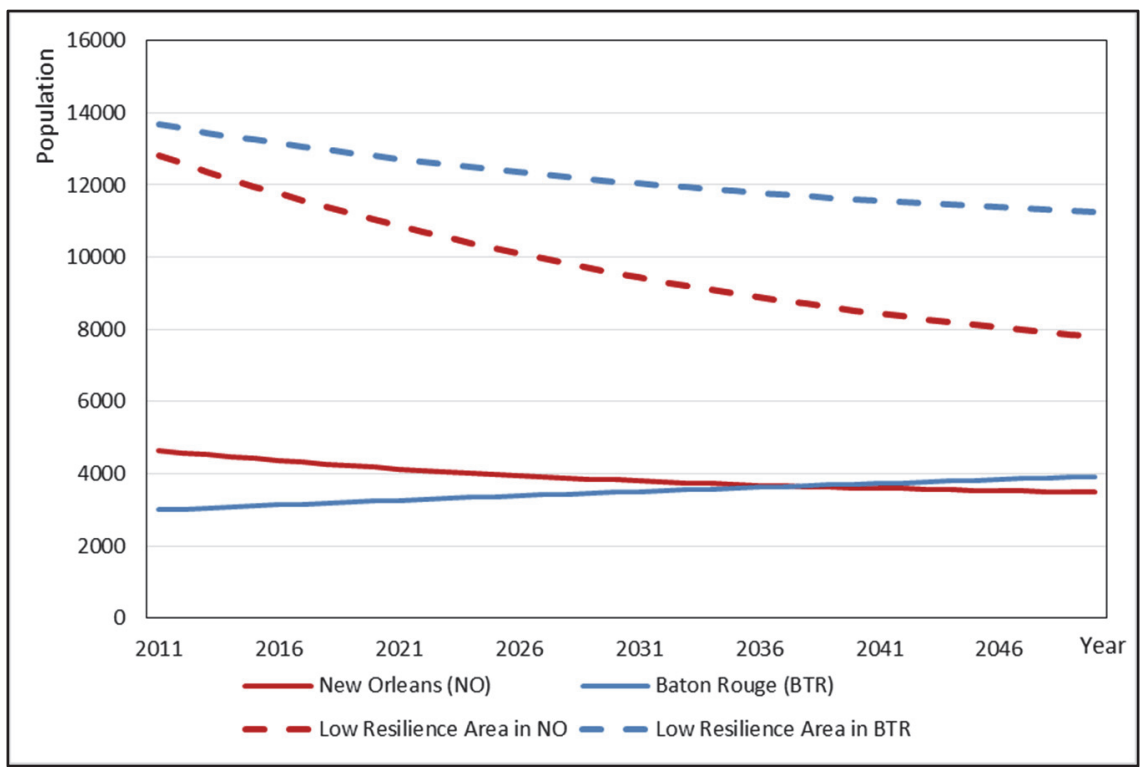

Figure 8.2 Average Simulated Population of the Low-Resilience Areas in New Orleans and Baton Rouge and the Whole City Areas of New Orleans and Baton Rogue from 2010 to 2050

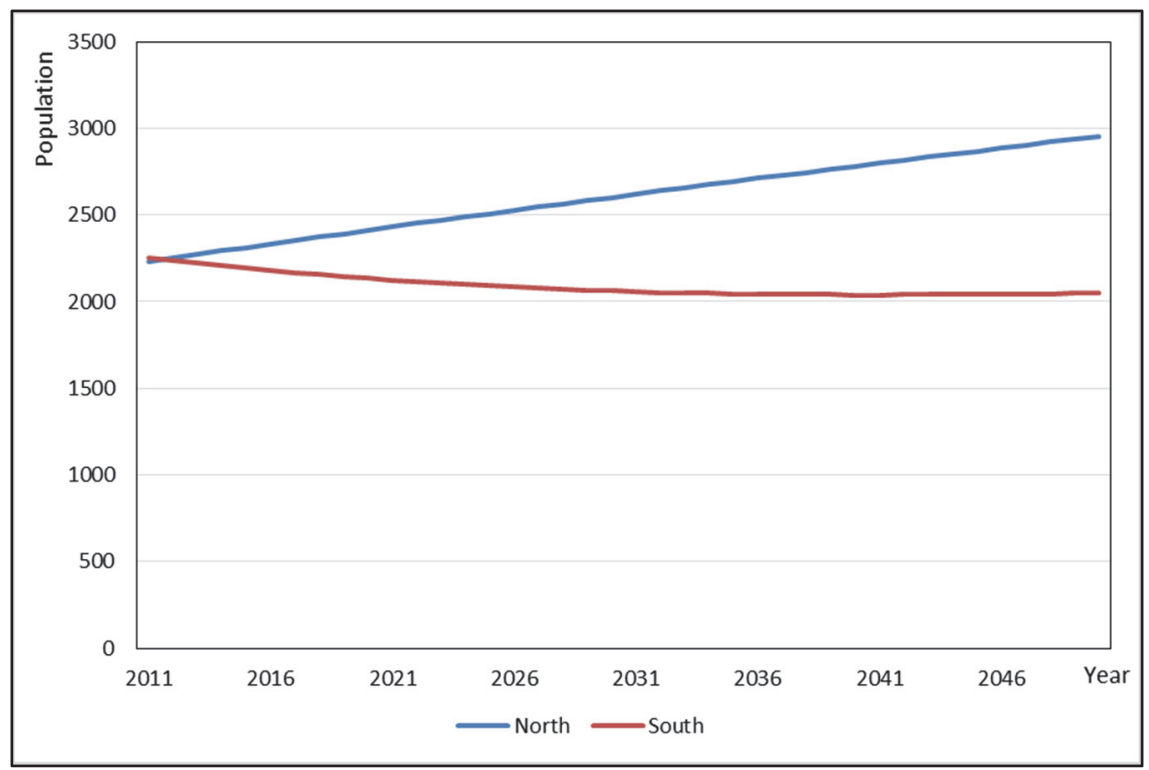

Figure 8.3 Average Simulated Population of the "North" City Areas and the "South" City Areas from 2010 to 2050 


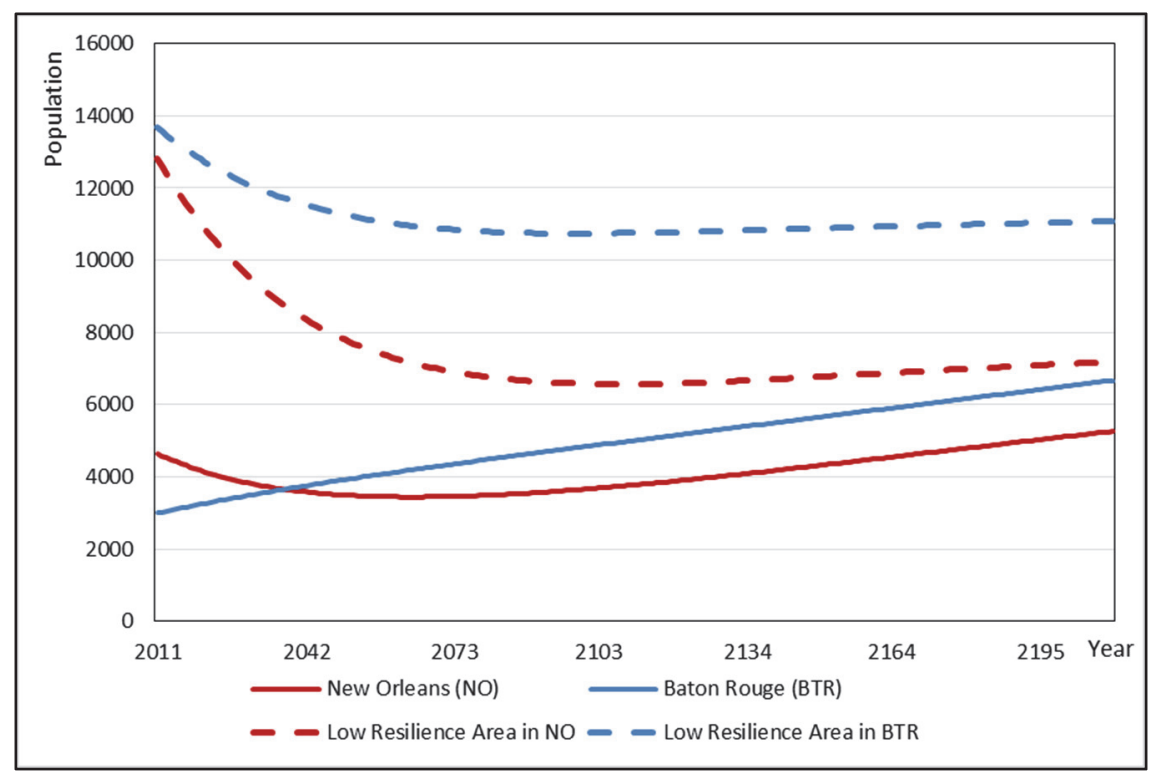

Figure 8.4 Average Simulated Population of the Low-Resilience Areas in New Orleans and Baton Rouge and the Whole City Areas of New Orleans and Baton Rogue from 2010 to 2210

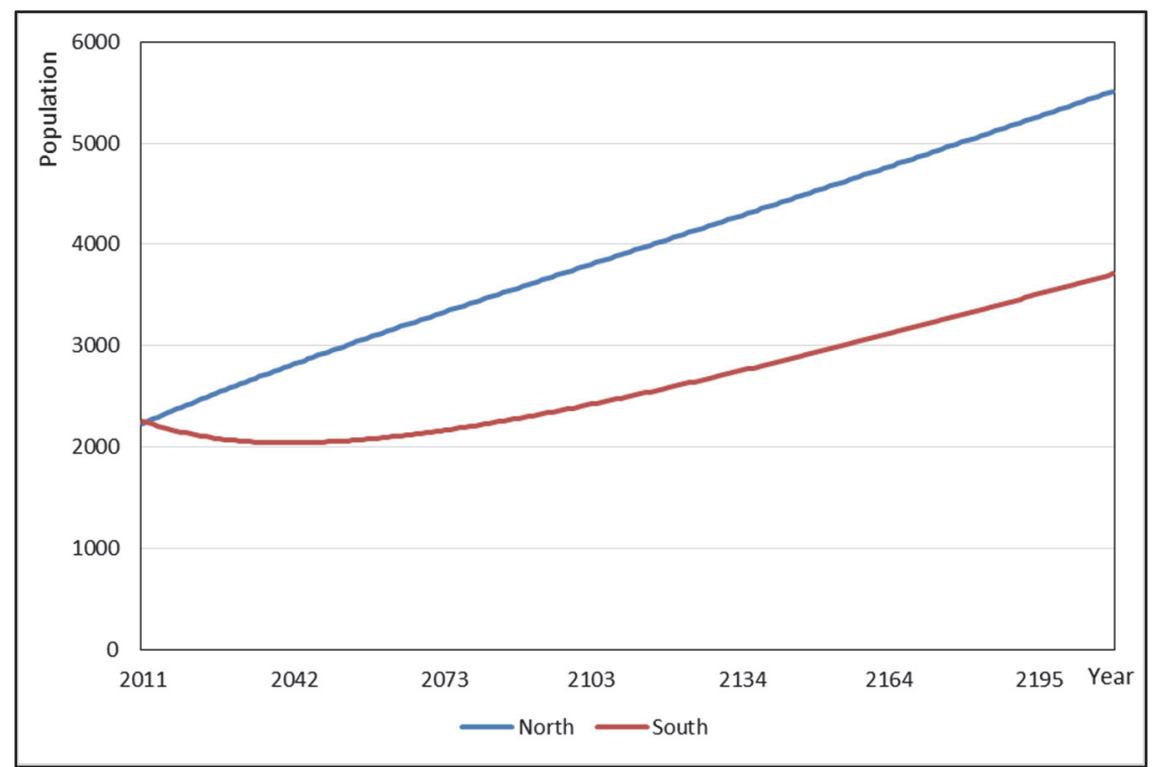

Figure 8.5 Average Simulated Population of the "North" City Areas and the "South" City Areas from 2010 to 2210

From Figure 8.2 and Figure 8.3, the average population of the "north" region and its major metropolitan area (Baton Rouge) keeps growing, whereas the average population of the "south" region and its major metropolitan area (New Orleans) keeps declining in the short time-period simulation. Most of the low-resilience areas (only except two spatial units) for both the "north" and the "south" region are located in the metropolitan areas of Baton Rouge 
and New Orleans, and their average populations are declining no matter if they are in the "north" or in the "south". The low-resilience areas in the "south" have their average population decreasing at a much faster speed than the areas in the "north". It should be noted that all the susceptible spatial units are in the "south".

For the long time-period simulation, although most of the low-resilience spatial units are located in the central metropolitan area of New Orleans, the average population of all the spatial units in New Orleans (Figure 8.4) does not go down all the time in the long time period simulation. Break points were observed in the average population time series of both the whole "south" region and the New Orleans metropolitan area. For the low-resilience areas (both from the "north" and from the "south), the decreasing speeds of their populations drop to 0 and their populations even get increased slightly in the long time-period simulation. This harbors the idea that even though the "south" areas suffered much hazard damages and the resultant population decreases, the whole area may still be able to find a certain equilibrium after two decades.

\subsection{Comparison of the Growth of the Developed Areas}

In terms of the coupled human and natural dynamics, one of the major human impacts on the environment is the increase in the developed land use areas. The time series of the average developed land use area percentage of both the "north" and the "south" were extracted from both the simulations for a short time-period (Figure 8.6) and a long time-period (Figure 8.7). Unlike the comparison of the populations, in which only the urban spatial units were

analyzed, the comparison of the developed land use areas takes all the spatial units into consideration. 


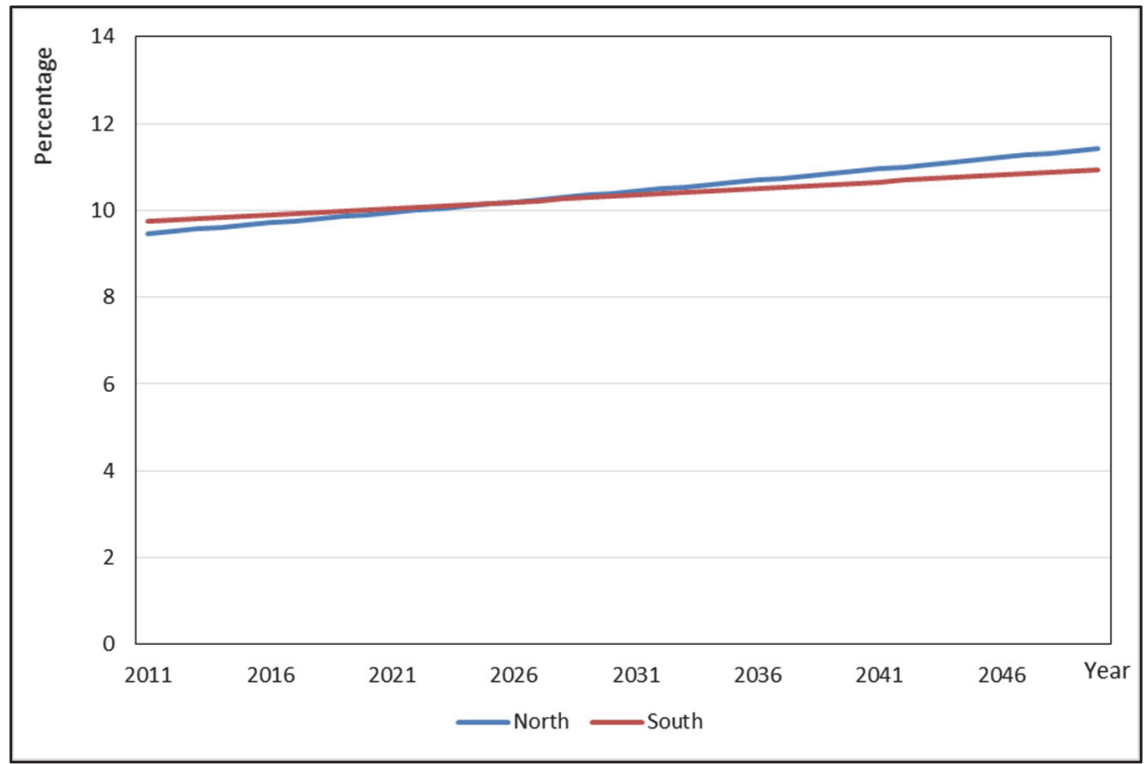

Figure 8.6 Average Simulated Developed Land Use Area Percentage of the "North" Areas and the "South" Areas from 2010 to 2050

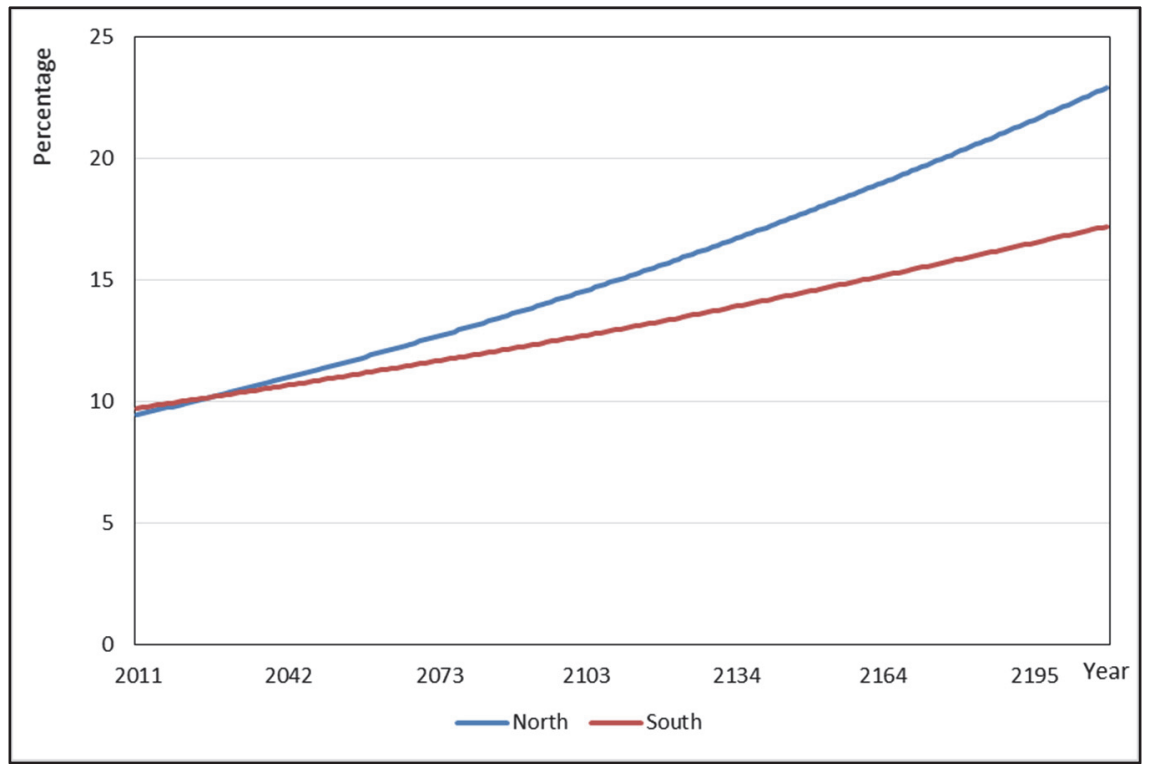

Figure 8.7 Average Simulated Developed Land Use Area Percentage of the "North" Areas and the "South" Areas from 2010 to 2210

From Figure 8.6 and Figure 8.7, the "south" region has a slightly higher developed land use area percentage than the "north" region at the beginning of the simulation (in the year 2010), however its developing speed is lower. In the short time-period simulation, the average developed land use area percentages of the "north" and the "south" region are about the same, with the "south" having a slightly higher initial value but surpassed by the "north" at the end 
of the simulation. In the long time-period simulation, the developing speed of the "south' falls behind the "north" a lot, and the difference is exacerbated over time.

In evaluating the human impacts on the environment, the land use types that would be sacrificed because of the expansion of the developed land use areas were analyzed. Since the long time-period simulation is mainly for observing the break points, and a long timeprediction generally has more uncertainty than a short time prediction, this analysis was only done for the short time-period simulation. For the short time-period simulation, the spatial units with at least $10 \%$ of their total areas converted to the developed land areas were identified and mapped in Figure 8.8. Most of these spatial units are located in the city boundaries. For the two major metropolitan areas (New Orleans and Baton Rouge) these spatial units tend to be at their peripheral city areas, whereas for the other city areas, these spatial units are more likely to be around their city centers. These identified spatial units were separated into two groups by the "north-south" boundary. The percentages of each land cover type in 2010 were calculated for the two groups and shown in Figure 8.9. 


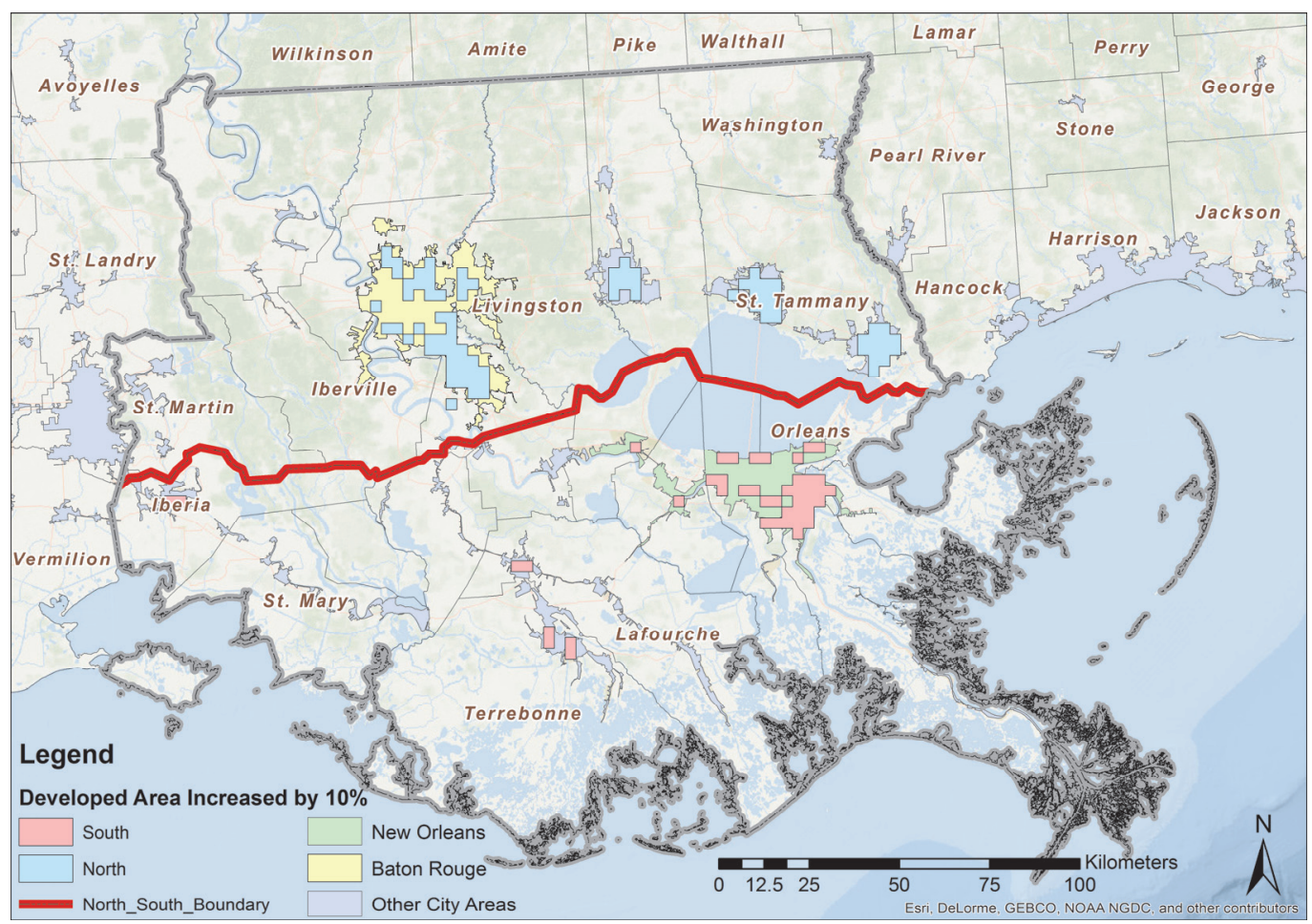

Figure 8.8 Areas with their Simulated Developed Land Percentages increased by at Least 10\%

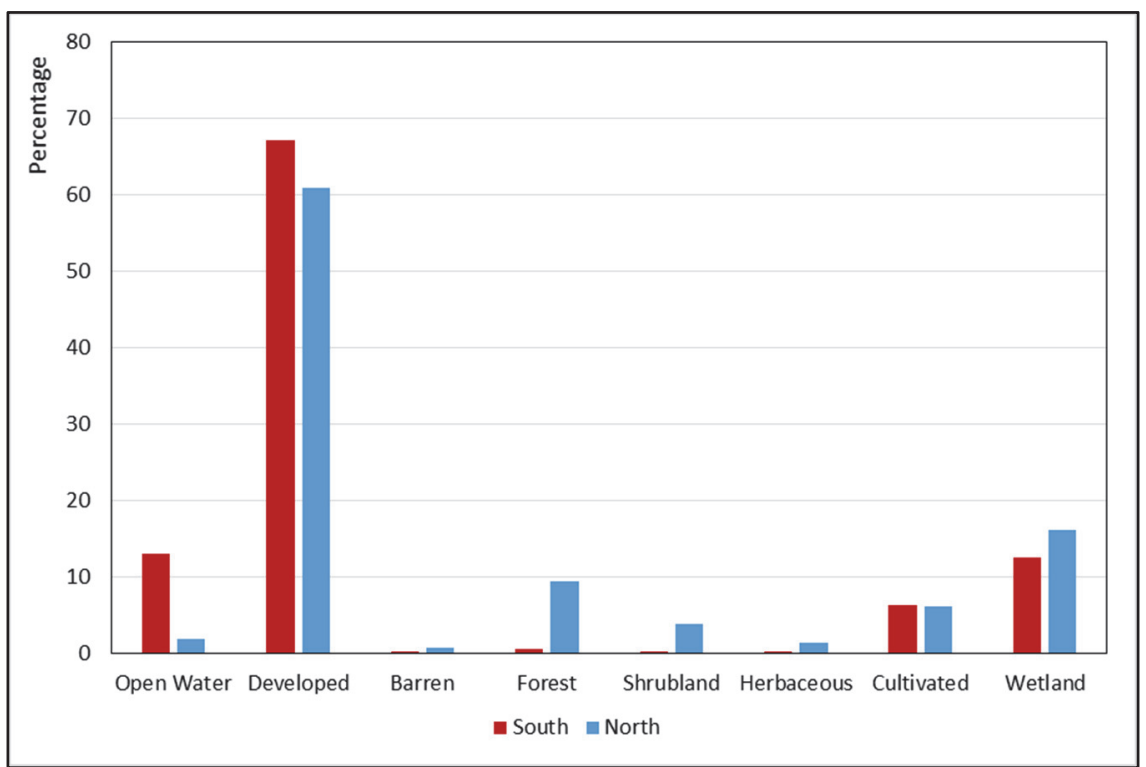

Figure 8.9 Percentages of Land Use Types of the North and the South Areas in 2010 with their Developed Land Percentages increased by at Least $10 \%$ by 2050

From Figure 8.9, for the spatial units with their developed land percentages increased by at least $10 \%$ by 2050 , located in both the "north" and the "south", the developed land is the dominant land type. This means that the urban expansion is most likely to occur in the areas 
already having a high percentage of urban area. High percentages of wetlands and cultivated lands (agricultural lands) also exist in these spatial units. This harbors the idea that for both the "south" and the "north", agricultural lands and wetlands are most likely to be sacrificed for urban growth. For these spatial units located in the "north", they also have high percentages of forests and grasslands. Thus, in the "north", the urban growth also has a high probability to trigger the deforestation. In the meantime, for these spatial units located in the "south", there is a high percentage of open water areas. It reflects that the urban expansion of the "south" region is closed to open water area.

\subsection{Comparison of the Relative Land Prices}

The average Relative Land Prices were also compared between the spatial units in the city areas of both the "north" and the "south". The average Relative Land Prices in the short time-period simulation and the long time-period simulation are shown in Figure 8.10 and Figure 8.11.

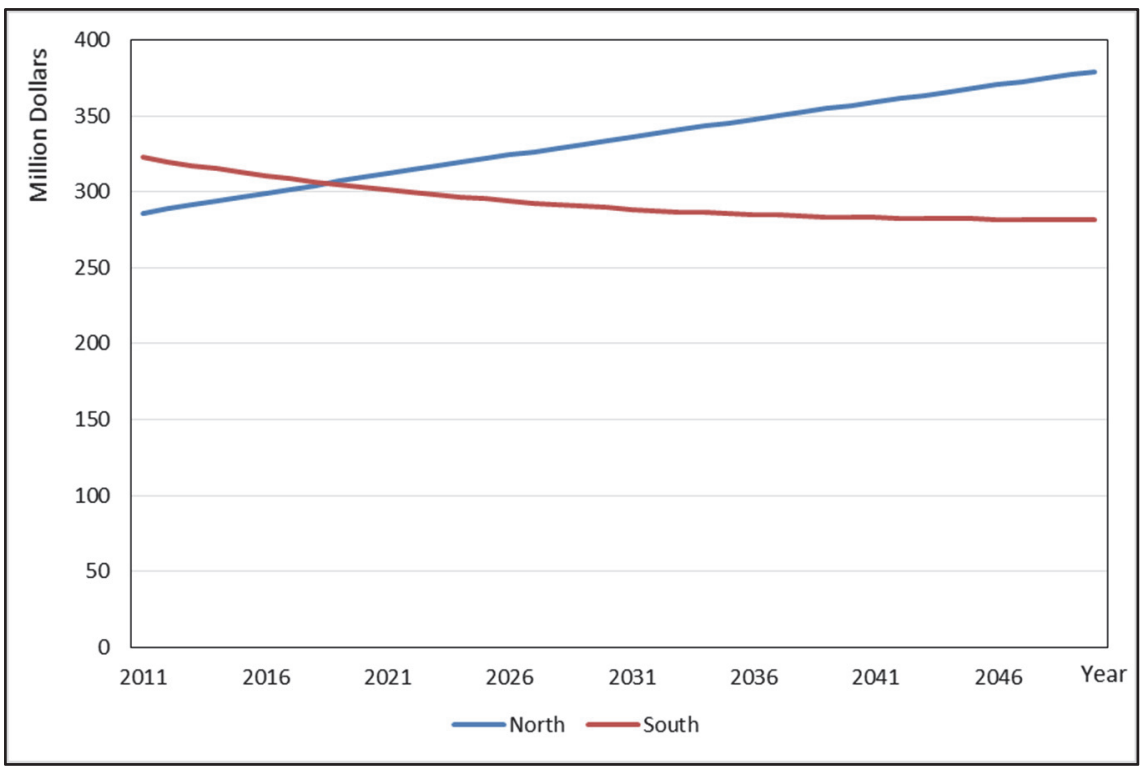

Figure 8.10 Average Simulated Relative Land Price of the "North" Areas and the "South" Areas from 2010 to 2050 


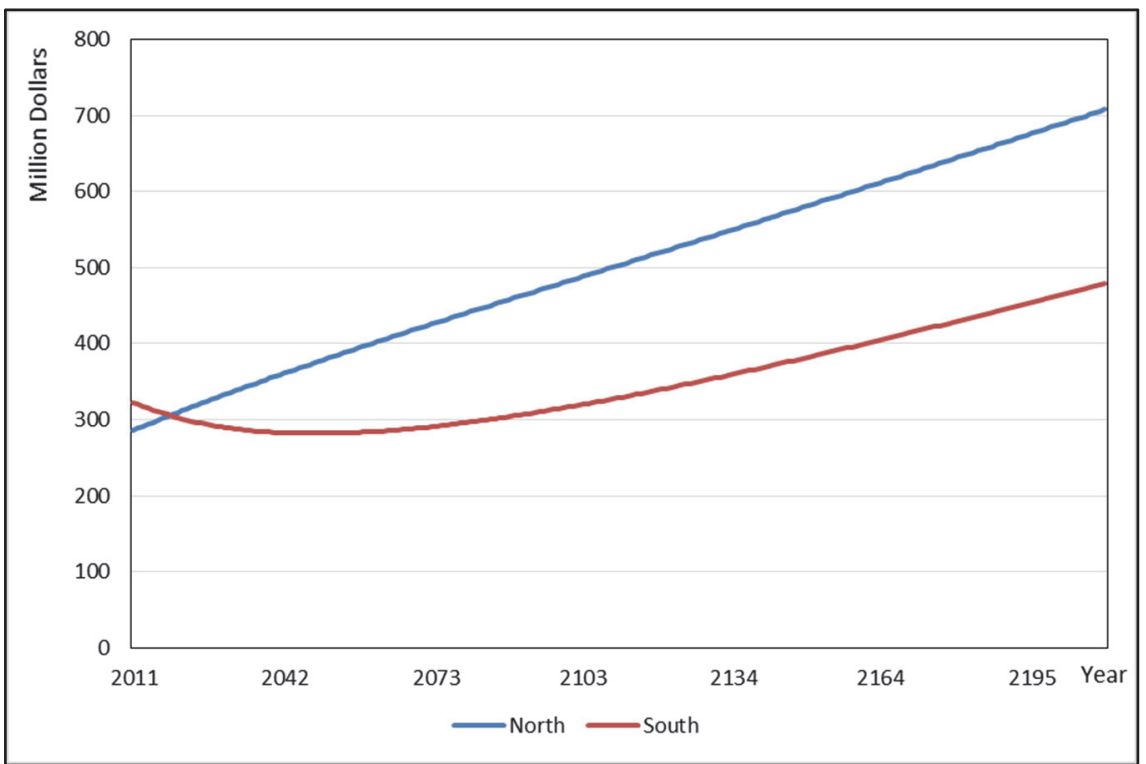

Figure 8.11 Average Simulated Relative Land Price of the "North" Areas and the "South" Areas from 2010 to 2210

From Figure 8.10 and 8.11 , for all the spatial units in the city areas (including both high-resilience and low-resilience spatial units), the changes of their Relative Land Prices are much like the changes of their populations, since population is one of the two variables in calculating the Relative Land Price. However in contrast with the population, the average Relative Land Prices of the "south" was much higher than the average of the "North" at the beginnings of the simulations. In the long time-period simulation, the average Relative Land Price of the "south" spatial units changed from the decreasing state into a steady increasing state. Similarly to the population growth and the developed land expansion, the Relative Land Price Growth of the "south" still falls behind those of the "north" in both the short and the long time-period simulations. 


\subsection{References}

Lam, N.S.N., Arenas, H., Pace, R.K., LeSage, J.P., Campanella, R. (2012). Predictors of business return in New Orleans after hurricane Katrina. PLoS One 7(10): 1-8.

Qiang, Y., Lam N.S.N. (2015). Modeling land use and land cover changes in a vulnerable coastal region using artificial neural networks and cellular automata. Environmental Monitoring and Assessment. 


\section{CHAPTER 9 : CONCLUSIONS}

This study developed a spatial dynamic model with the situated spatial units as the modeling unit of analysis to predict the population changes and the developed land use percentage changes. Updating rules for the spatial units were extracted by using the Elastic Net regression functions, and Genetic Algorithms were used to calibrate the parameters for the neighborhood effects. Based on the predicted population changes from 2010 to 2050, a resilience assessment model inspired by the RIM model was built by using the Self-Organizing Map method to classify the resiliency of the spatial units. Four groups of spatial units with their resilience capacities ranked from high to low (usurper, resistant, recovering, and susceptible) were identified. The sustainability analysis was based on the criterion that if the population of a spatial unit can "sustain" by maintaining a certain percentage of its initial population after a number of years. Three conditional spaces have been identified from the sustainability assessment: the tipping space, the mitigatable space, and the sustainable space. A Relative Land Price model was defined to indicate the relative values of the spatial units due to their combinations of population and utility. The projected populations, developed land areas, and Relative Land Prices were compared between the two groups of spatial units of the “north' and the "south" for two simulation periods (2010 to 2050 and 2010 to 2210).

\subsection{Modeling Discussion}

The system dynamic model built in this study focused on uncovering the social and economic emergences in a vulnerable coastal area, which does not result from the existence of a central controller. Such emergences had already been observed from 2000 to 2010 (e.g., population decreased in the "south" region and population increased in the "north" region). The system dynamics derived in this study has been proven as an effective method to monitor the existence of such emergences due to its ability of keeping the values of the variables 
updated. Some emergences were observed in the predicting simulation (e.g. the average population of the south will change from decreasing to increasing after about 50 years). In terms of the coupled natural and human dynamics in this region, this model mainly focused on changes of the system due to the human components. The dynamics of environmental variables were not modeled in the simulation, such as the elevation, subsidence rate, and the open water percentage change (land loss rate). However, the same modeling methodology can be used to uncover the dynamics of these natural variables when their driving factors have been empirically and scientifically identified (e.g., accretion rate, biomass diversity, land building process). In this research, only the developed land use area percentage is modeled. With more natural variables incorporated, the other types of land cover (e.g., land loss) can also be simulated. Adding the natural part in the model will make the model more comprehensive, and a better understanding of the complex coupled human and natural dynamics of this coastal area can be obtained.

Elastic Net was used to extract the relations among the variables, thus the accuracy of Elastic Net determines the accuracy of the final spatial dynamic model. It should be noted that a "white-box" data mining such as Elastic Net is needed in this study, since the internal structure of the data mining method should be known to extract the rules for building the system dynamics. Elastic Net was chosen in this study due to the ease of extracting rules from it, especially when a linear Elastic Net is used as in this study. It should be noted that the Elastic Net built here used a space-for-time substitution for generating sample data points. By doing so it assumed that the dependency of each dependent variable on each independent variable is the same among all the spatial units. The model could be improved if it is without this spacefor-time substitution and the resultant assumption, but this needs more data for each spatial unit, which means that there should be several temporal data points for each variable in each spatial unit. Due to the data availability at the time when conducting this study, this assumption has to be made for creating the Elastic Net model. 
The Genetic Algorithm did improve the accuracy of the model, and proved the existence of the neighborhood effects to some extents in the final calibrated model. In building a "bottom-up" model, parameters setting is one of the most important task. Exhaustively trying out all the possibilities of parameter combinations is unrealistic due to the extremely high computational time cost. Technically, any optimization algorithm can be used to conduct the task in this step. The Genetic Algorithm as an Evolutional Algorithm was used in this study, because it was found to lead to a quick convergence on tuning the parameters. However, the final calibrated parameters are unexpected, with no direct neighborhood effects on population and utility $\left(P_{1}=0\right.$ and $\left.P_{3}=0\right)$. Although a sensitivity analysis was conducted to confirm this result, this calibration part needs to be revisited in a future research. In this study, the final model only has $P_{2}$ equal to 0.5 , which indicates a low level of feed-backs among the spatial units, and almost linear outputs for the subsequent analysis. Different types of neighborhood filters other than the Moore filter used in this study, different fitness functions as well as the different initializations of the GA, could be tested in the future research.

\subsection{Results Discussion}

The results of this study can be summarized into three aspects: the resilience results, the sustainability results, and the Relative Land Price results, all of which came from the simulation results through running the spatial dynamic model under different scenarios.

The resilience assessment depends on the simulated population change. Spatial units identified as usurper and resistant are high resilience spatial units, whereas spatial units identified as recovering and susceptible are low resilience spatial units. The resilience assessment was only on the spatial units in the city areas of the study area (totally 901 spatial units). Out of the 901 spatial units, 54 were classified as low-resilience, and 847 were classified as high-resilience. The low-resilience spatial units were found to concentrate in the New Orleans metropolitan areas, and they suffered much more property damages from natural hazards and population loss from 2000 to 2010 than other spatial units, and their historical 
utility values (from 2000 to 2010) were also much lower. However, it should be noted that the low-resilience areas had larger initial populations in contrast with other areas, which makes the sustainability planning of these areas more important.

In this study, resilience is regarded as an ability to bounce back from external disturbance, or in other words, it reflects the largest magnitude of suffering external disturbance without losing the ability to bounce back. It is a short term expression and prerequisite of sustainability, and sustainability is considered a long term response of resilience. A spatial unit having a high resilience means that it has a possibility to be sustainable, but it does not necessarily mean that it will be sustainable. The sustainability assessment in this study depends on the specific sustainability goal. The sustainability goal in this study was set to be maintaining a certain percentage of the initial population of a spatial unit. The sustainability analysis in this study was to find out whether the spatial units of different resilience capacities will be sustainable under different hazard exposure scenarios for a given sustainability goal. From the results, the high-resilience spatial units will always be sustainable, and they have different degrees of population growths even without any mitigation plan based on the scenarios generated in Chapter 6. For the low-resilience spatial units, three sustainability conditions have been identified for different scenarios under a hypothetical mitigation budget: tipping space in which the simulated population will never reach the sustainability goal, mitigatable space in which whether the sustainability goal can be achieved depends on the actual mitigation cost, and the sustainable space in which the low-resilience spatial units will suffer less hazard damages and be sustainable.

Relative Land Price was defined in this study to indicate the relative values of the spatial units. The calculation of it depends on population and utility (Chapter 7). It is noted that in the calculation function, Relative Land Price is positively correlated with population but negatively correlated with utility. This does not mean that the higher the utility the lower the land price. Since utility and population are correlated, a spatial unit with high utility generally has high population. This means that for two spatial units with the same population, the one 
with lower utility has higher Relative Land Price and vice versa. This can be explained as that the spatial unit with higher utility should have had more population than the other, but if it still have the same population as the other, then it means that it has lower Relative Land Price. In terms of the relationship between resilience and Relative Land Price, two findings should be noted. First, it was found that for a fixed population and utility, the higher the resilience, the higher the Relative Land Price. Second, it was also found that for the actual utility and population combinations of the spatial units in the study area, the low-resilience areas have high Relative Land Prices mainly due to their high populations.

\section{3 "North" and "South" Discussion}

The "south" region has a similar average population as the "north" region in the year 2010 when the projecting simulations started, as well as a slightly higher average developed land area percentage and average Relative Land Price. However under the scenario with historical data, the "south" region fell behind the "north" region in the growth rates of all the three aspects. In the short-term simulation (2010-2050), the three aspects were all decreasing at the beginning, due to their high property damages suffered from natural hazards. However, in the long-term simulation (2010-2210), the "south" is able to maintain at a certain equilibrium, with steady growth in all the three aspects. This means even if the "south" areas still suffer the same property damages as the period from 2000 to 2010 (it should be noted that there was a catastrophic Katrina in this time period which was relatively rare), the population of these areas will not decrease all the time. Instead, they will be able to bounce back to a certain population level. 


\subsection{References}

Gunderson, L. Holling, C.S. (2002). Panarchy: Understanding transformations in human and natural systems. Washington, DC: Island Press. 


\section{APPENDIX A}

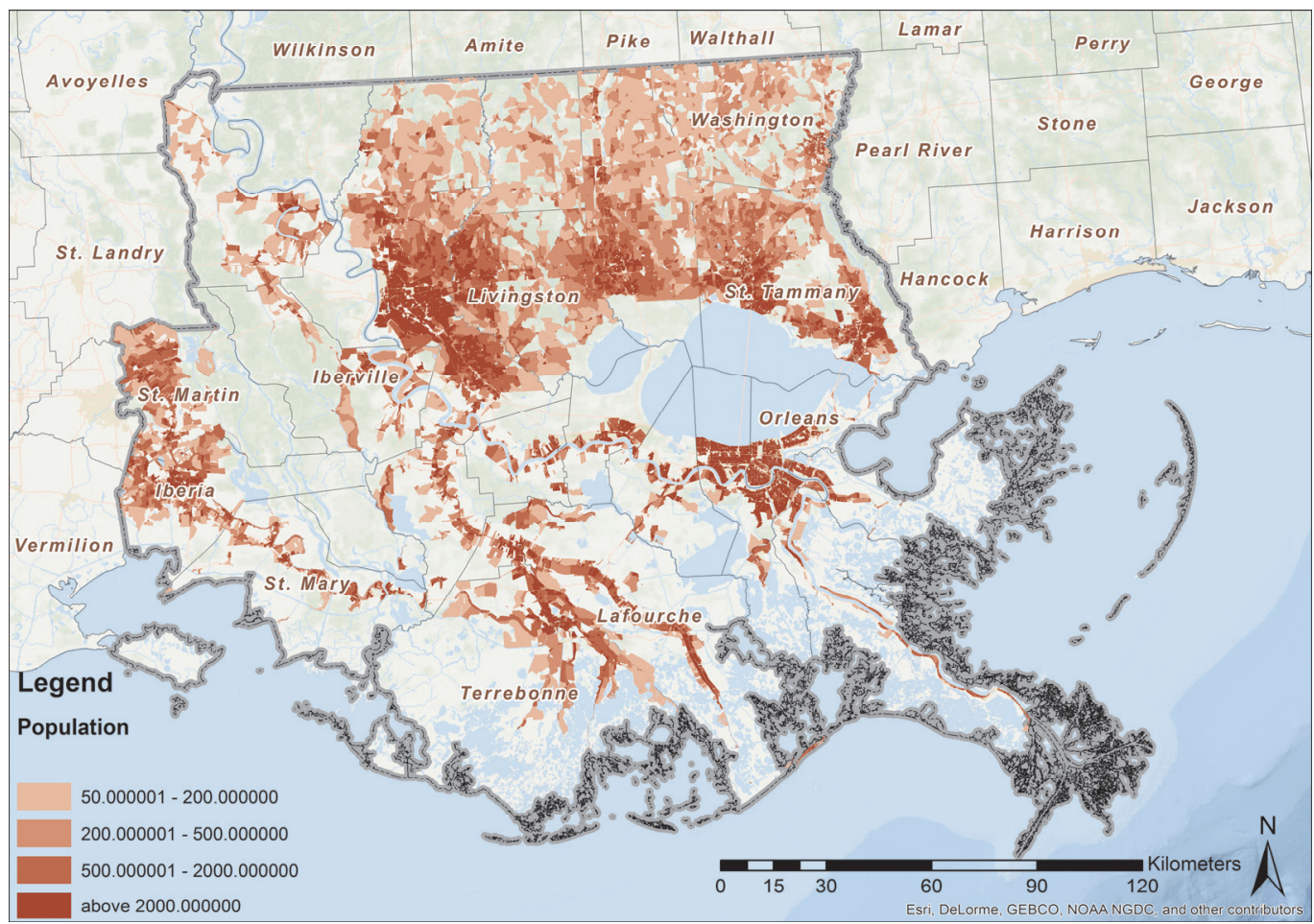

Figure A1 Population Density before Areal Interpolation in 2010

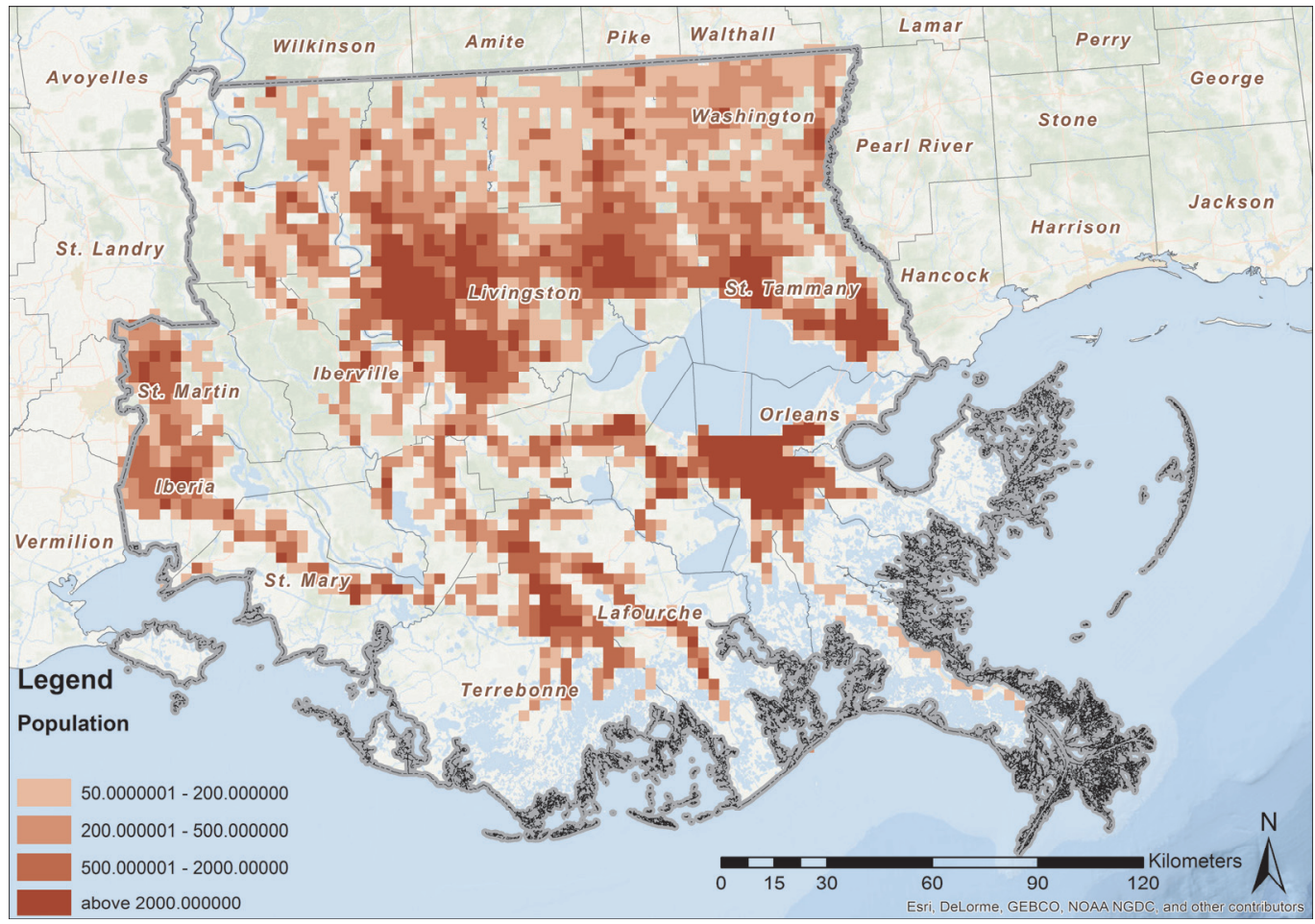

Figure A2 Population Density after Areal Interpolation in 2010 


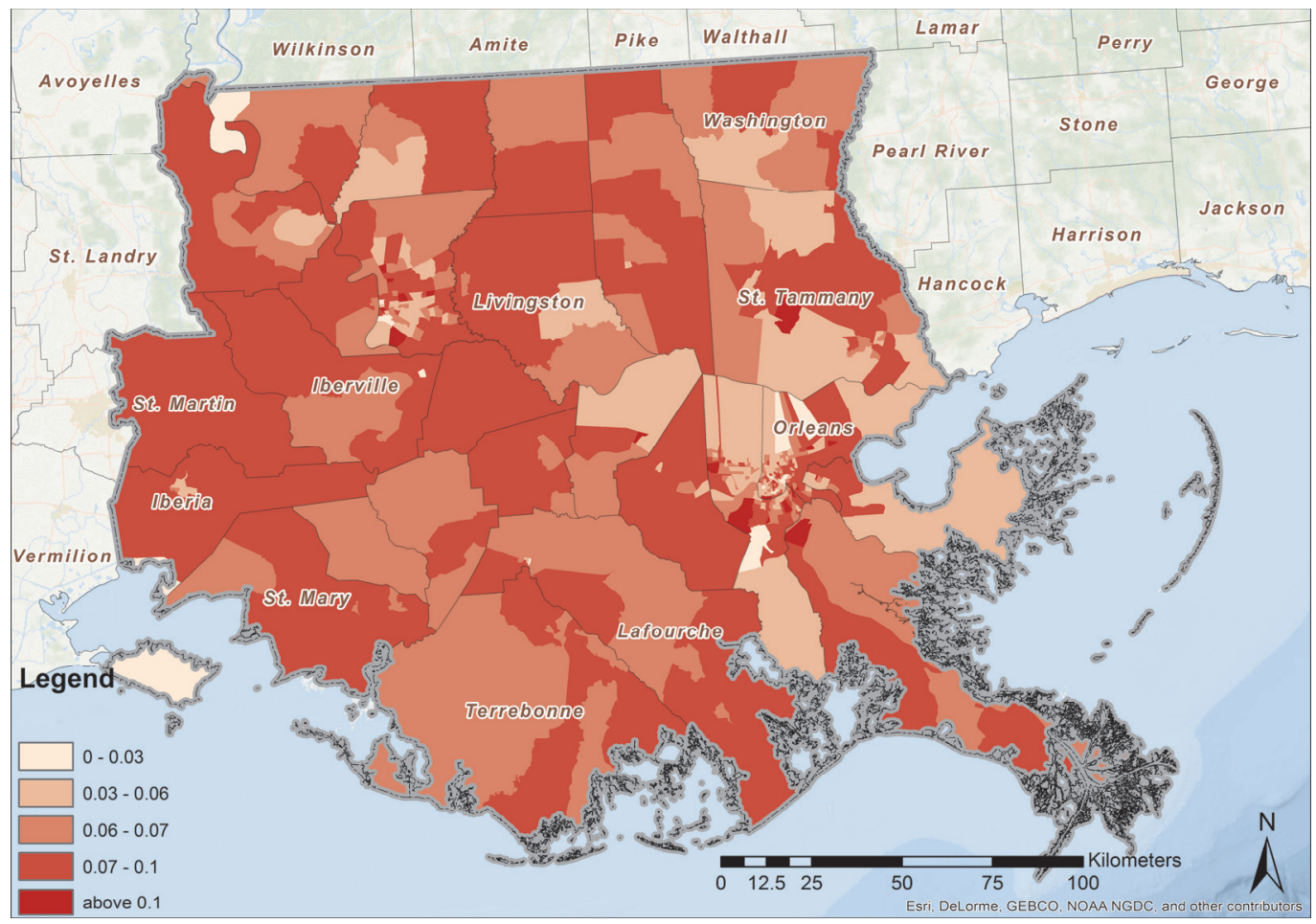

Figure A3 Percentage of Population under 5 Years Old before Areal Interpolation in 2000

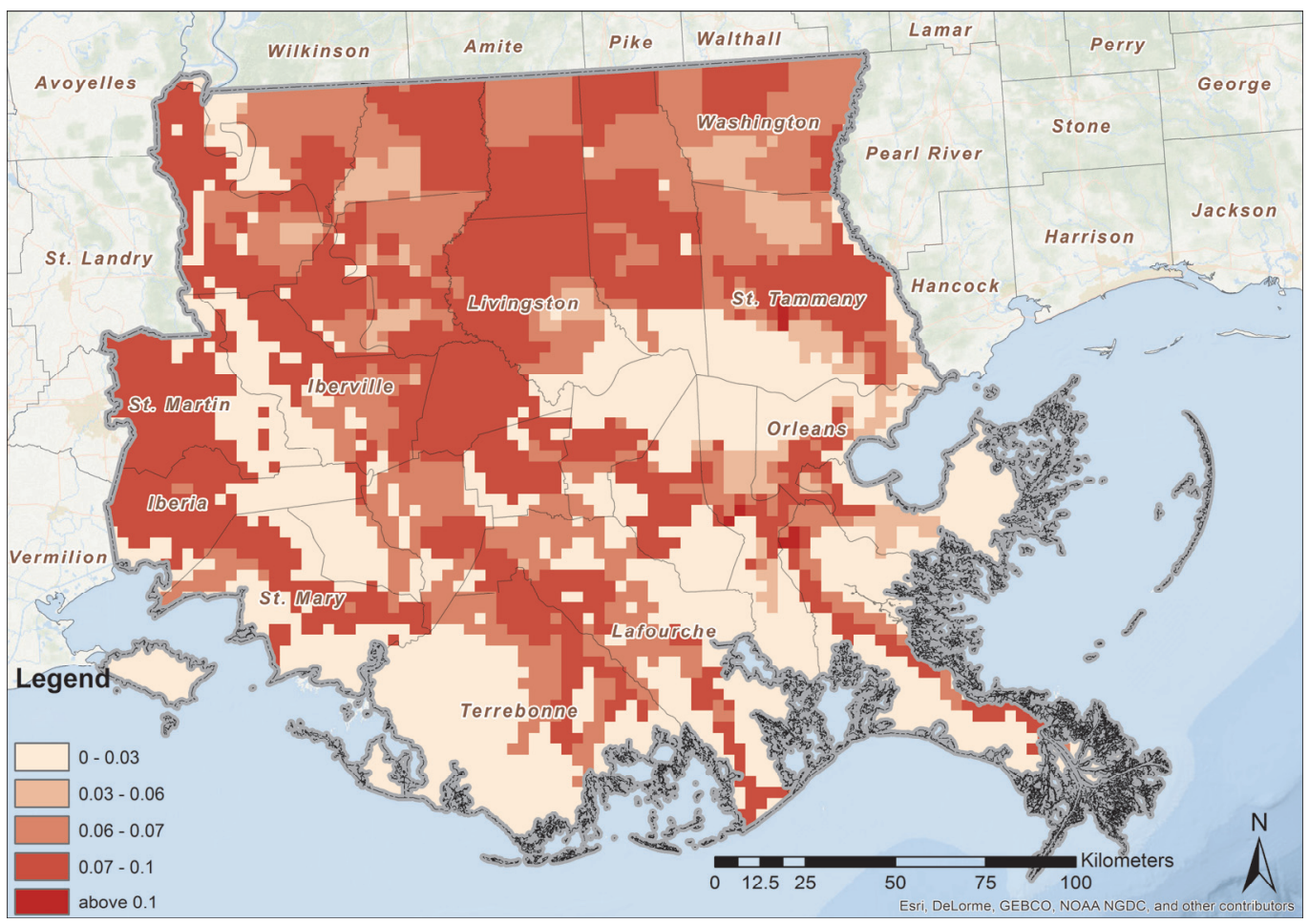

Figure A4 Percentage of Population under 5 Years Old after Areal Interpolation in 2000 


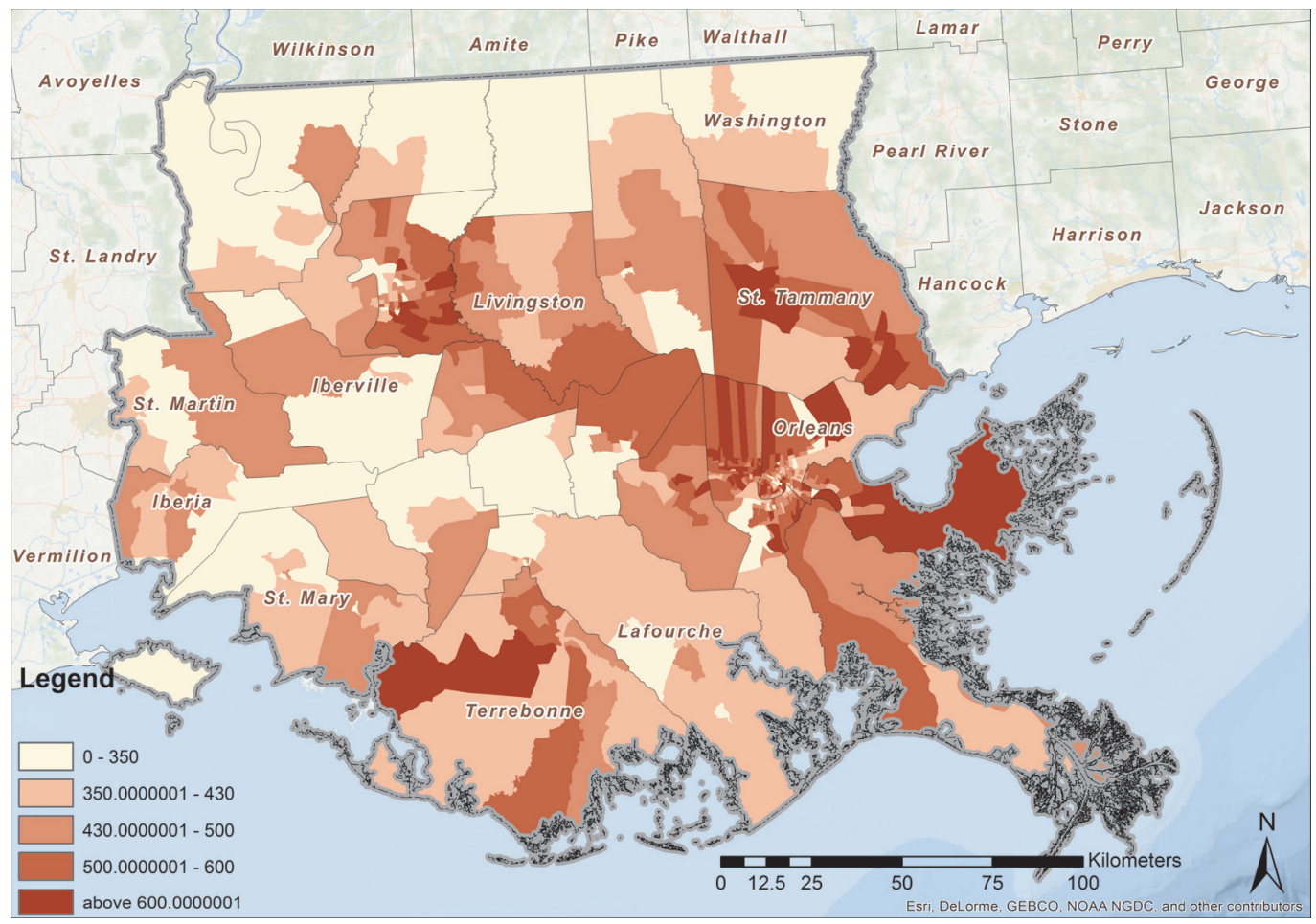

Figure A5 Median Gross Rent before Areal Interpolation in 2000

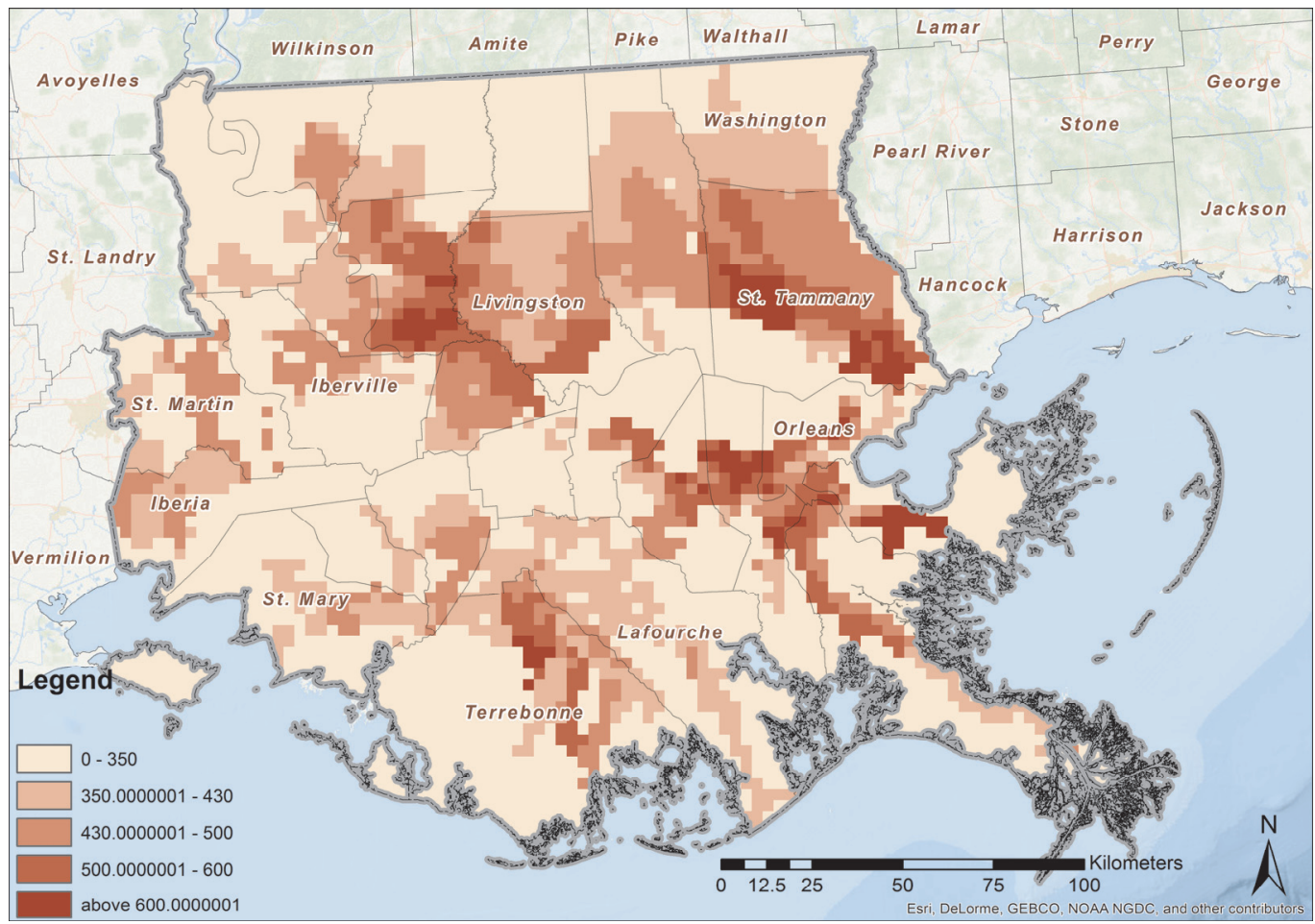

Figure A6 Median Gross Rent after Areal Interpolation in 2000 


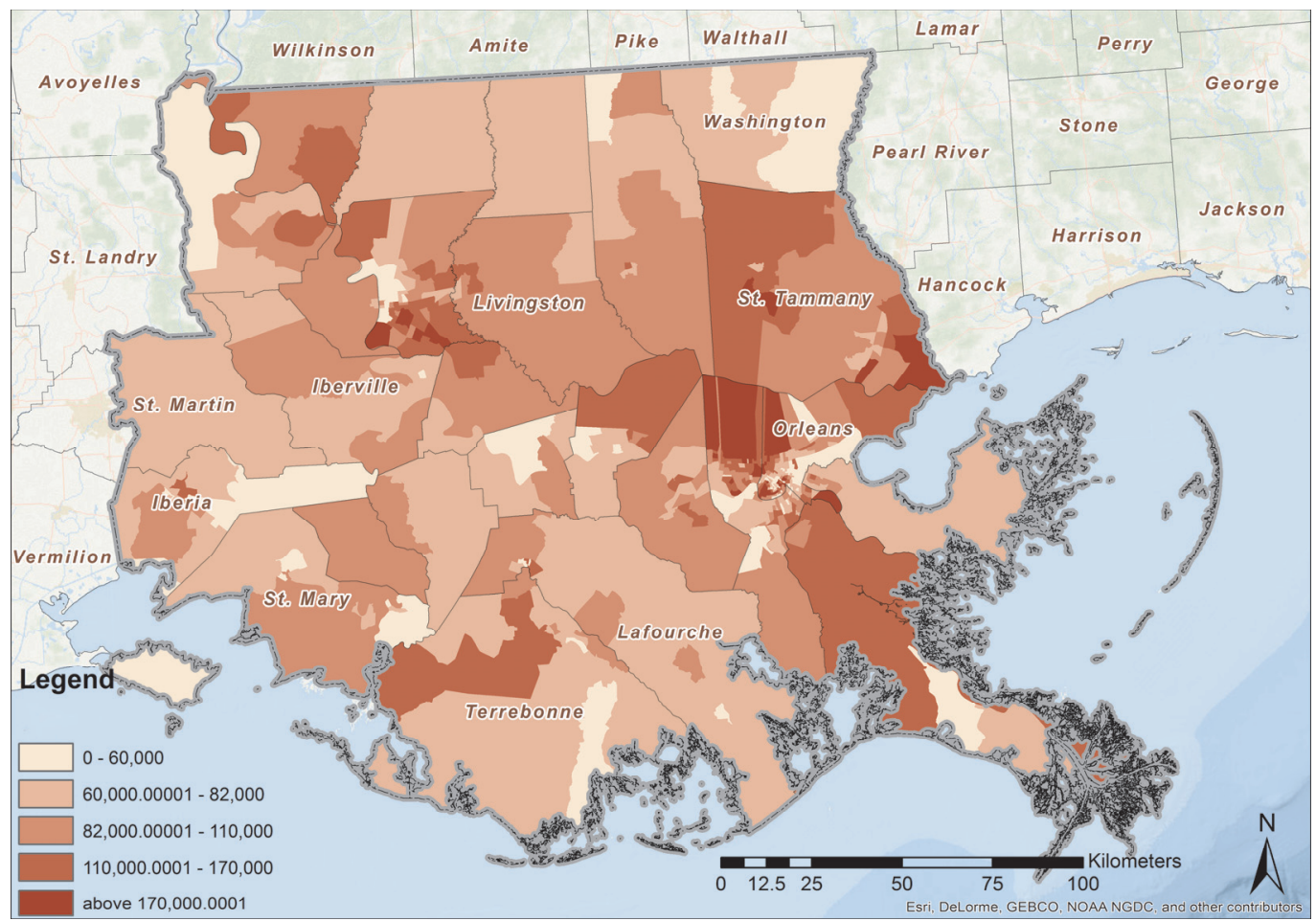

Figure A7 Median House Value before Areal Interpolation in 2000

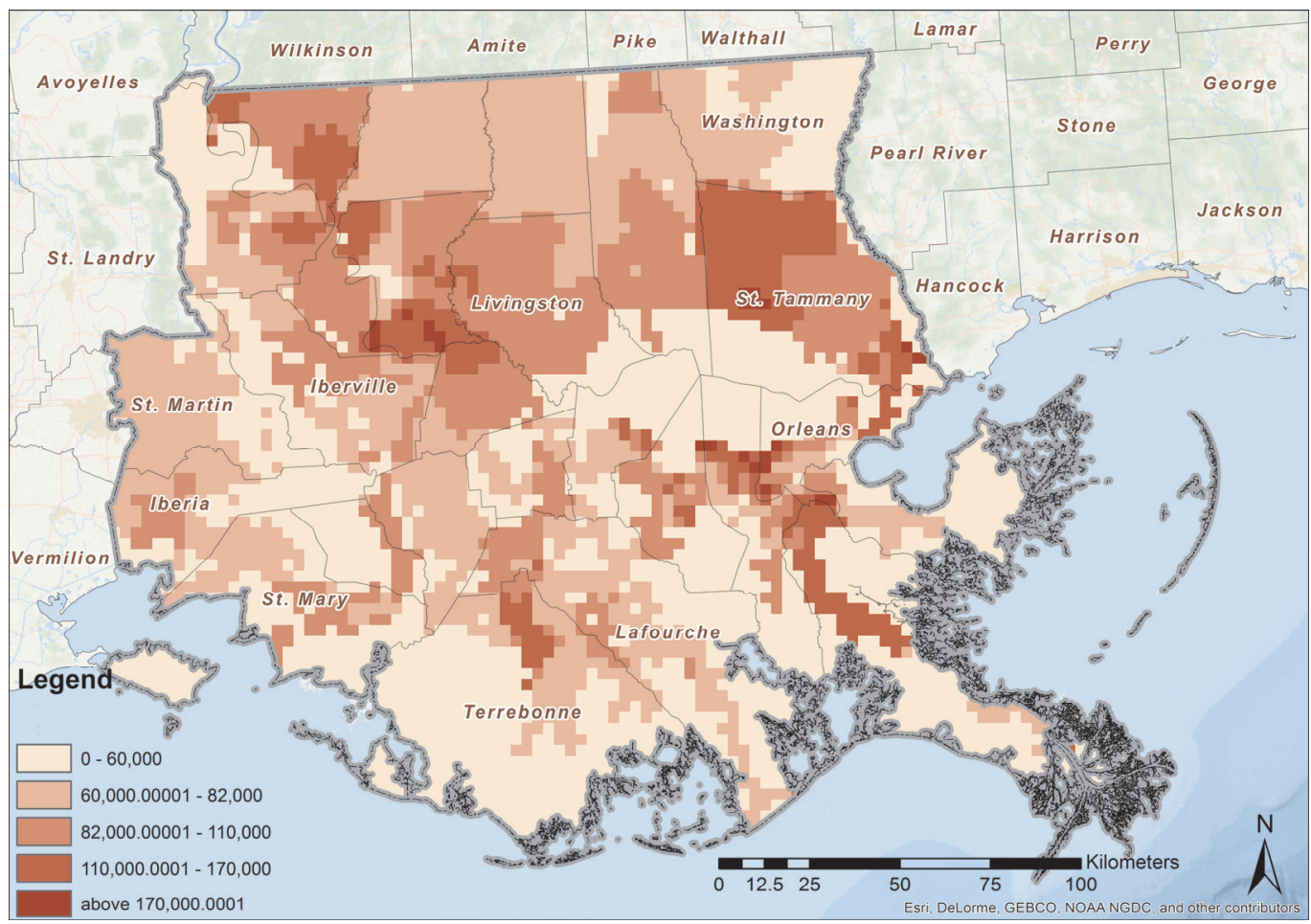

Figure A8 Median House Value after Areal Interpolation in 2000 


\section{APPENDIX B}

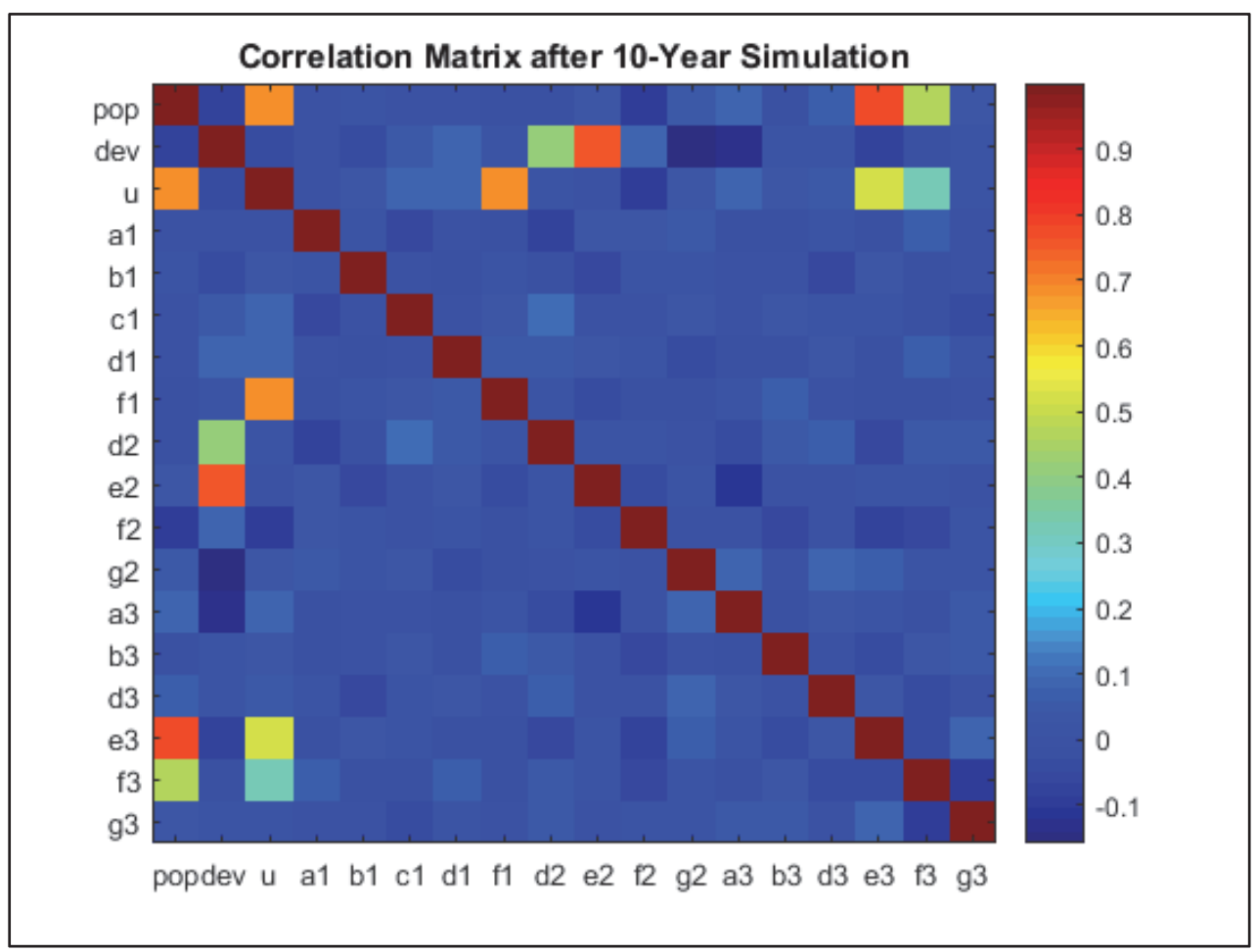

Figure B1 Correlations between the Parameters and the Sensitivity Assessment Variables after 10-Year Simulation

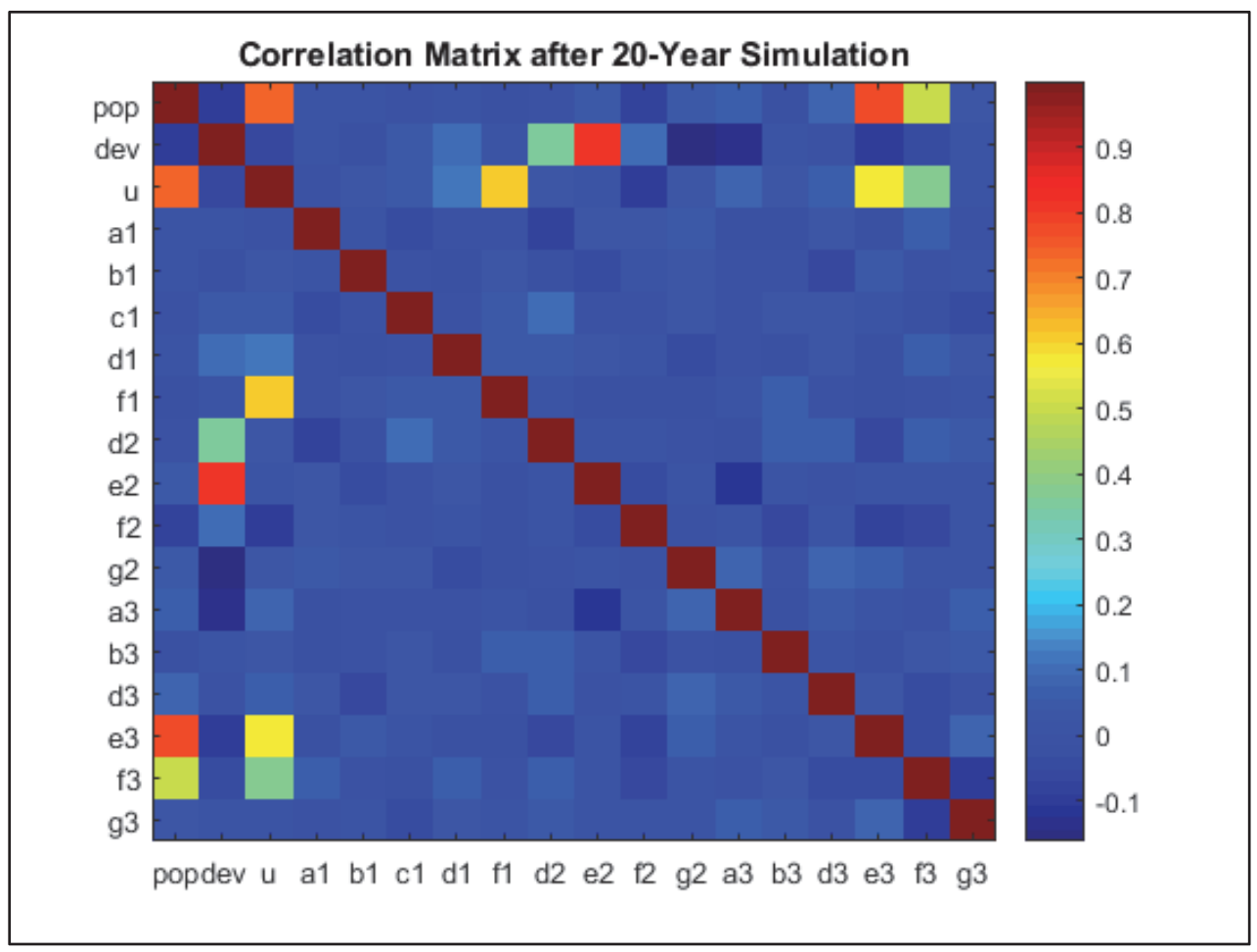

Figure B2 Correlations between the Parameters and the Sensitivity Assessment Variables after 20-Year Simulation 


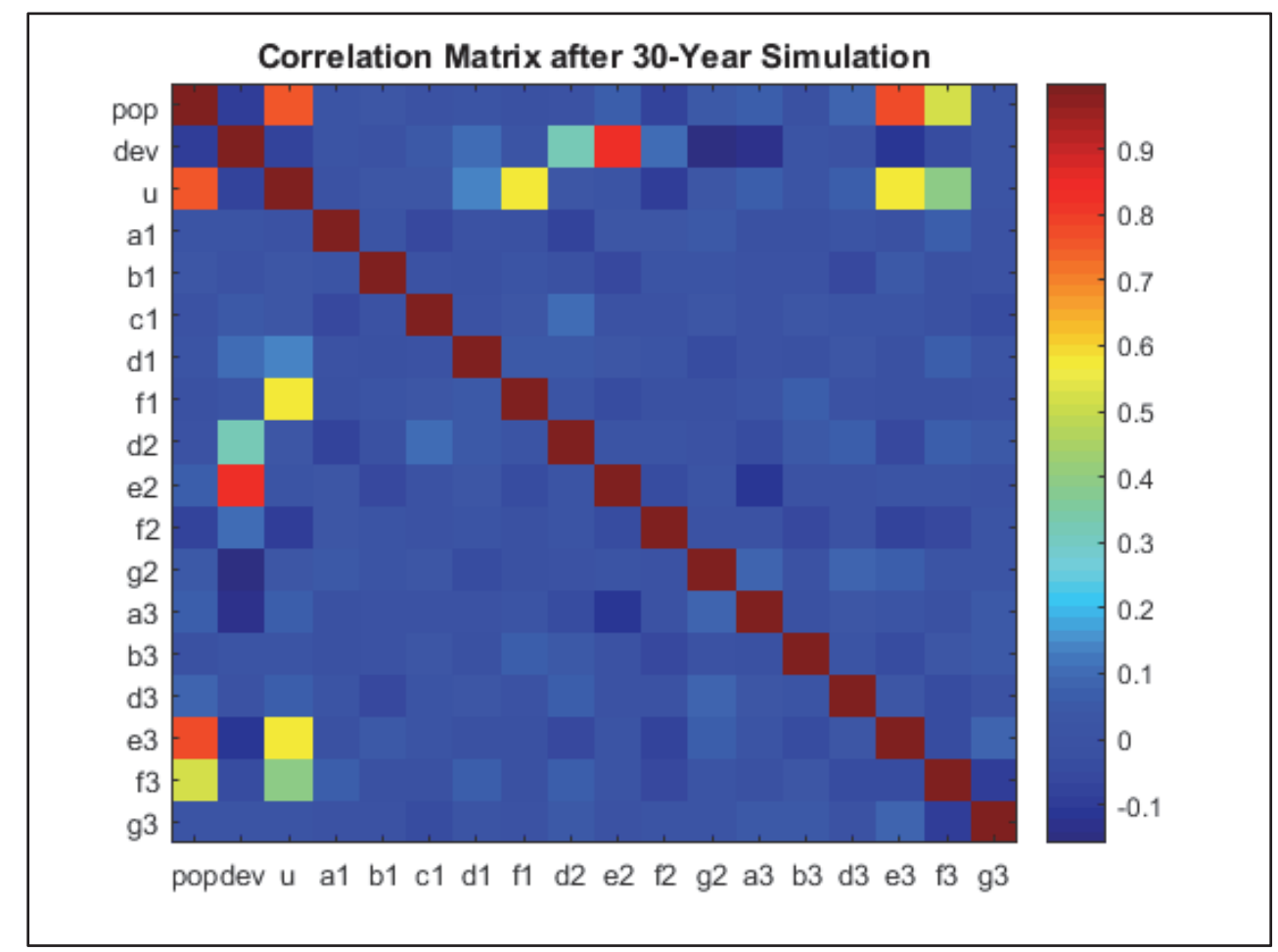

Figure B3 Correlations between the Parameters and the Sensitivity Assessment Variables after 30-Year Simulation

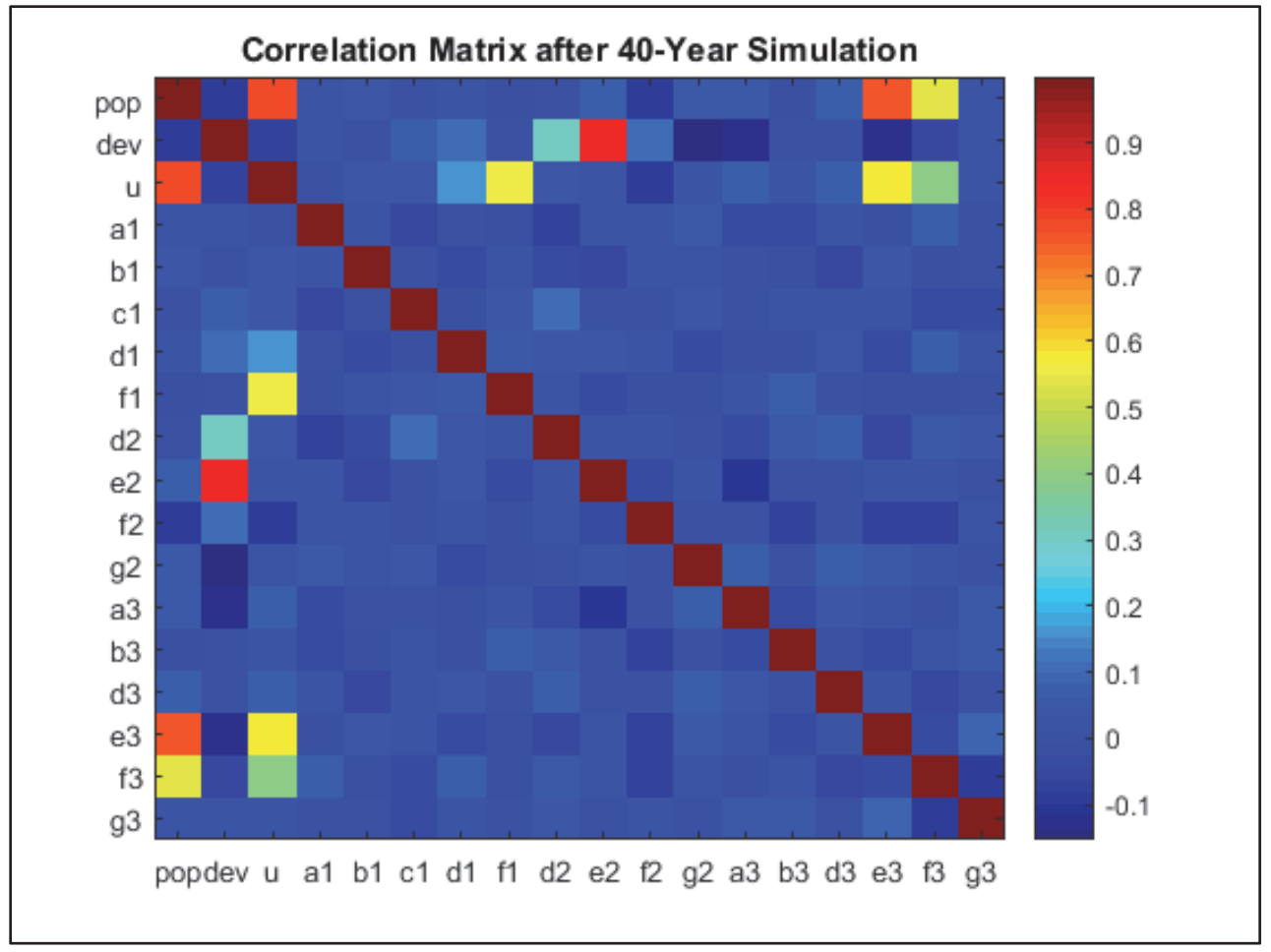

Figure B4 Correlations between the Parameters and the Sensitivity Assessment Variables after 40-Year Simulation 


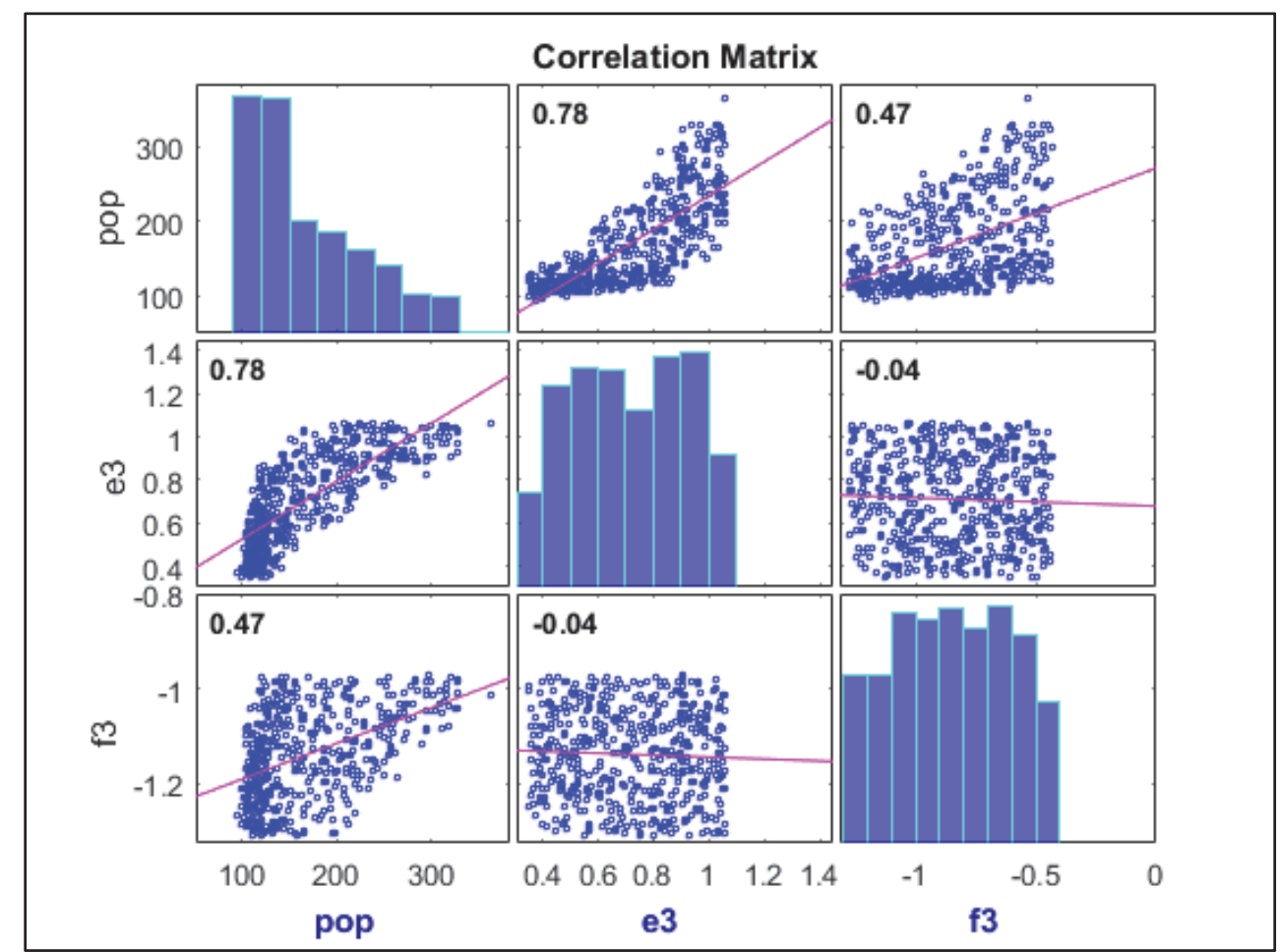

Figure B5 Histograms, Scatter Plots, and Correlation Coefficients for the Total Population and its High-Sensitivity Parameters after 10-Year Simulation (Standardized Value)

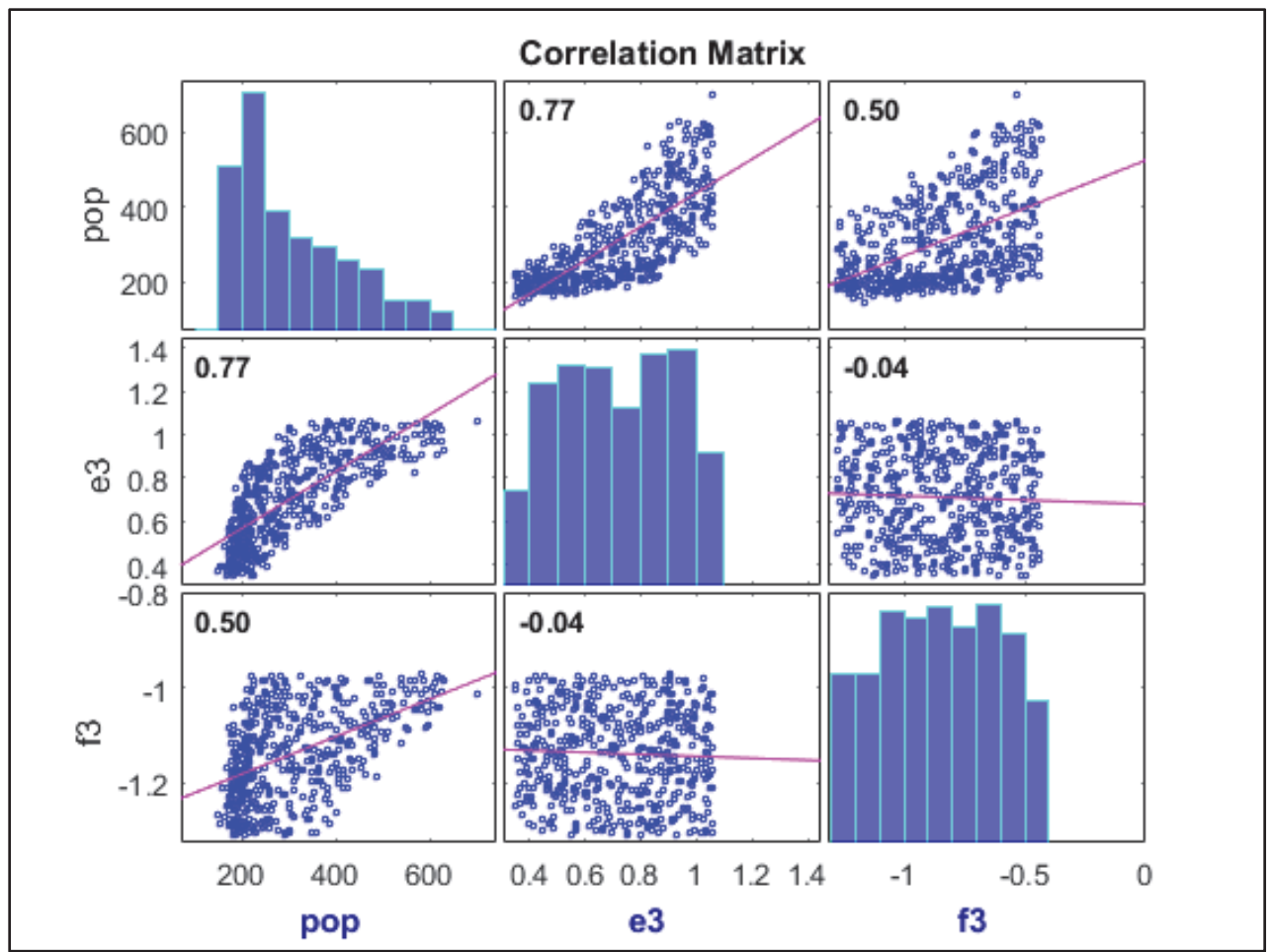

Figure B6 Histograms, Scatter Plots, and Correlation Coefficients for the Total Population and its High-Sensitivity Parameters after 20-Year Simulation (Standardized Value) 


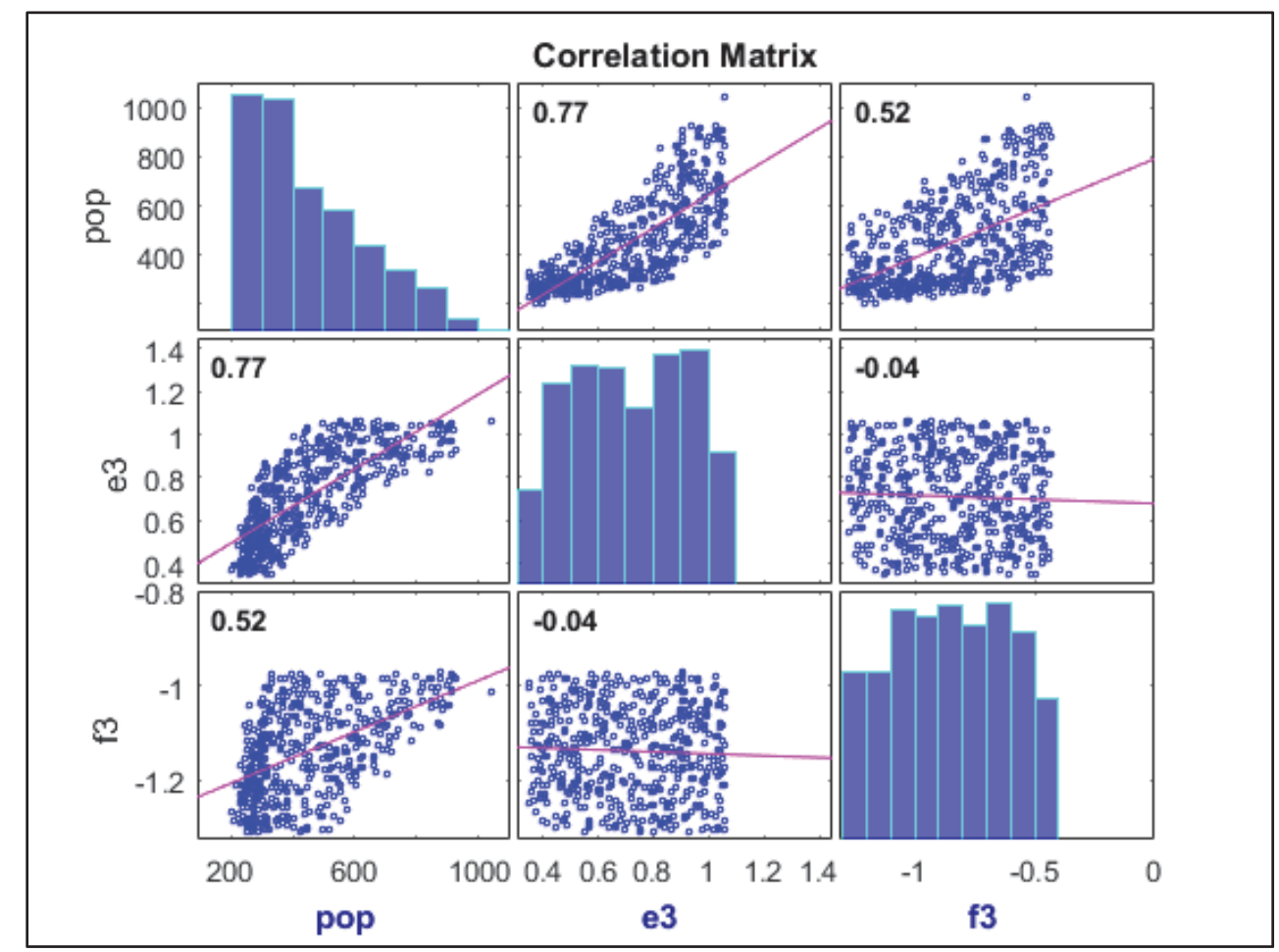

Figure B7 Histograms, Scatter Plots, and Correlation Coefficients for the Total Population and its High-Sensitivity Parameters after 30-Year Simulation (Standardized Value)

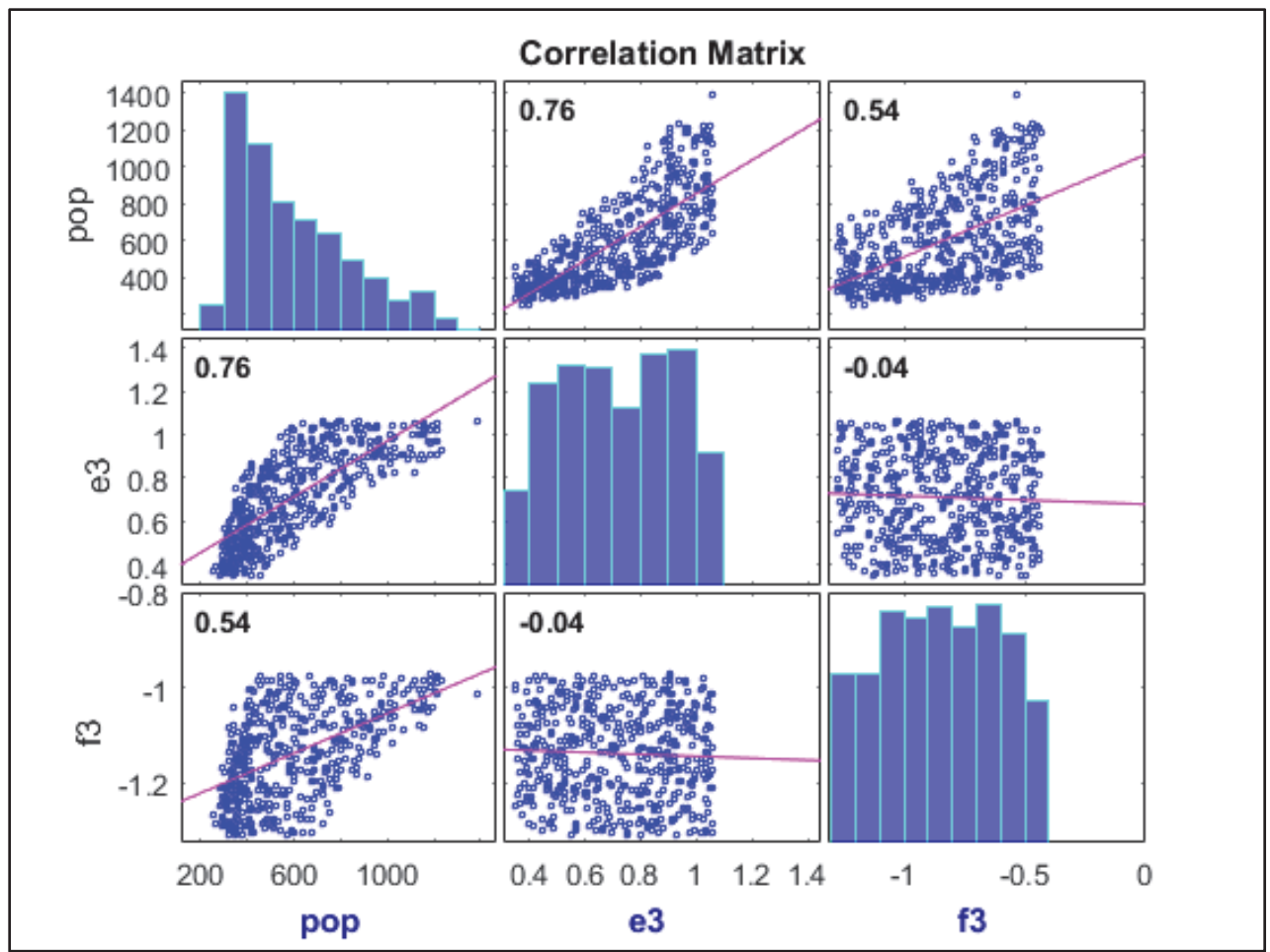

Figure B8 Histograms, Scatter Plots, and Correlation Coefficients for the Total Population and its High-Sensitivity Parameters after 40-Year Simulation (Standardized Value) 


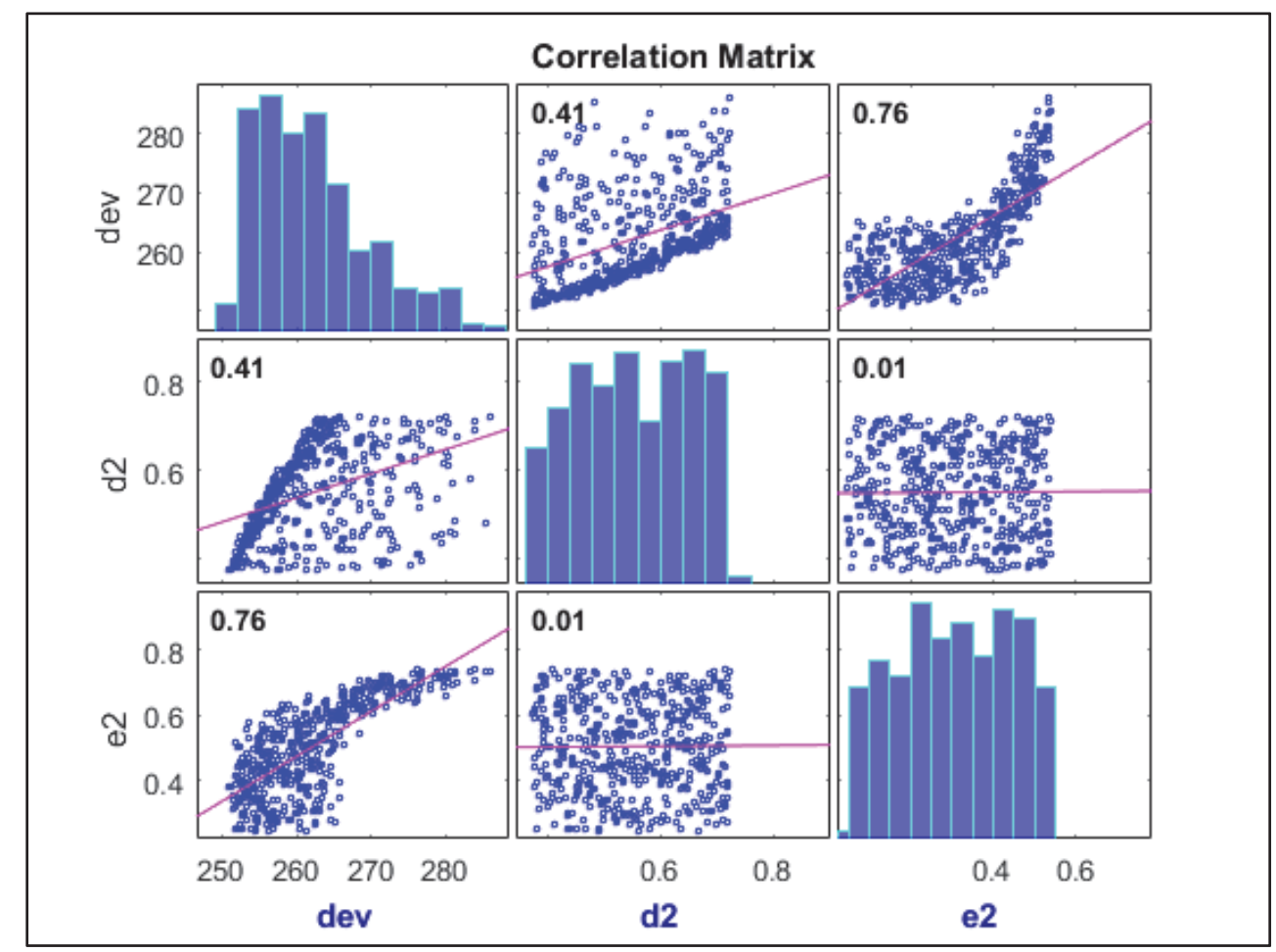

Figure B9 Histograms, Scatter Plots, and Correlation Coefficients for the Total Developed Land Area Percentage and its High-Sensitivity Parameters after 10-Year Simulation (Standardized Value)

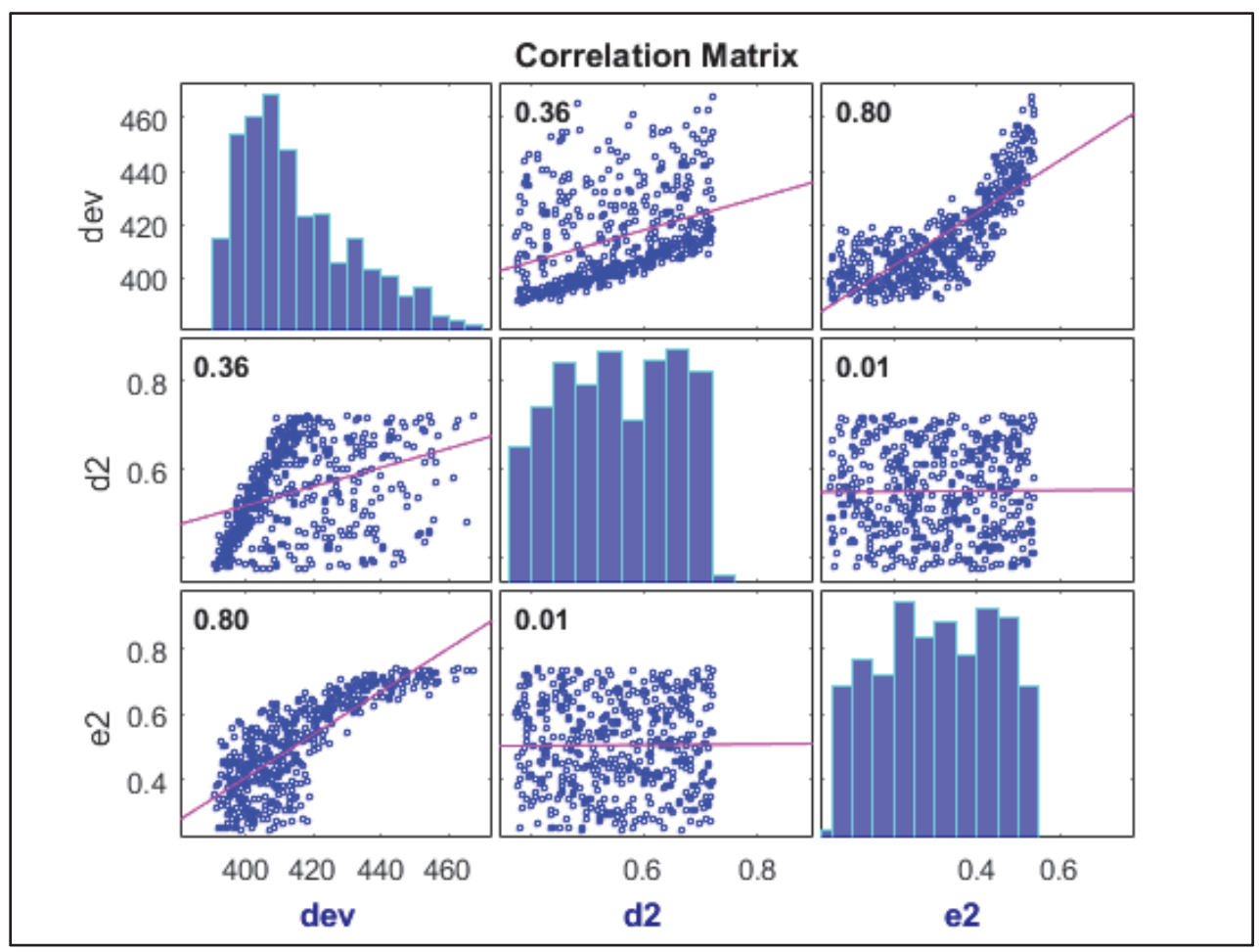

Figure B10 Histograms, Scatter Plots, and Correlation Coefficients for the Total Developed Land Area Percentage and its High-Sensitivity Parameters after 20-Year Simulation (Standardized Value) 


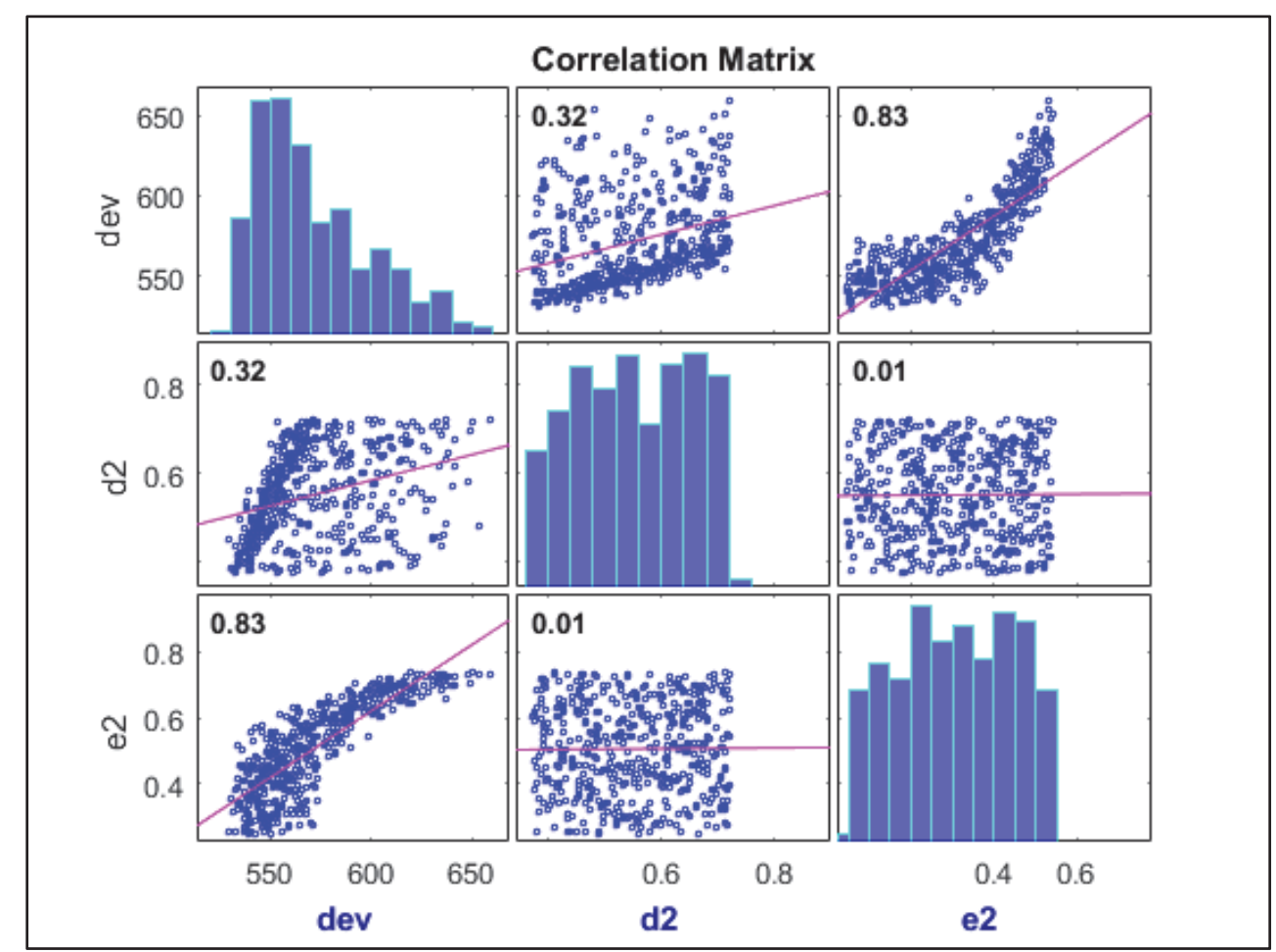

Figure B11 Histograms, Scatter Plots, and Correlation Coefficients for the Total Developed Land Area Percentage and its High-Sensitivity Parameters after 30-Year Simulation (Standardized Value)

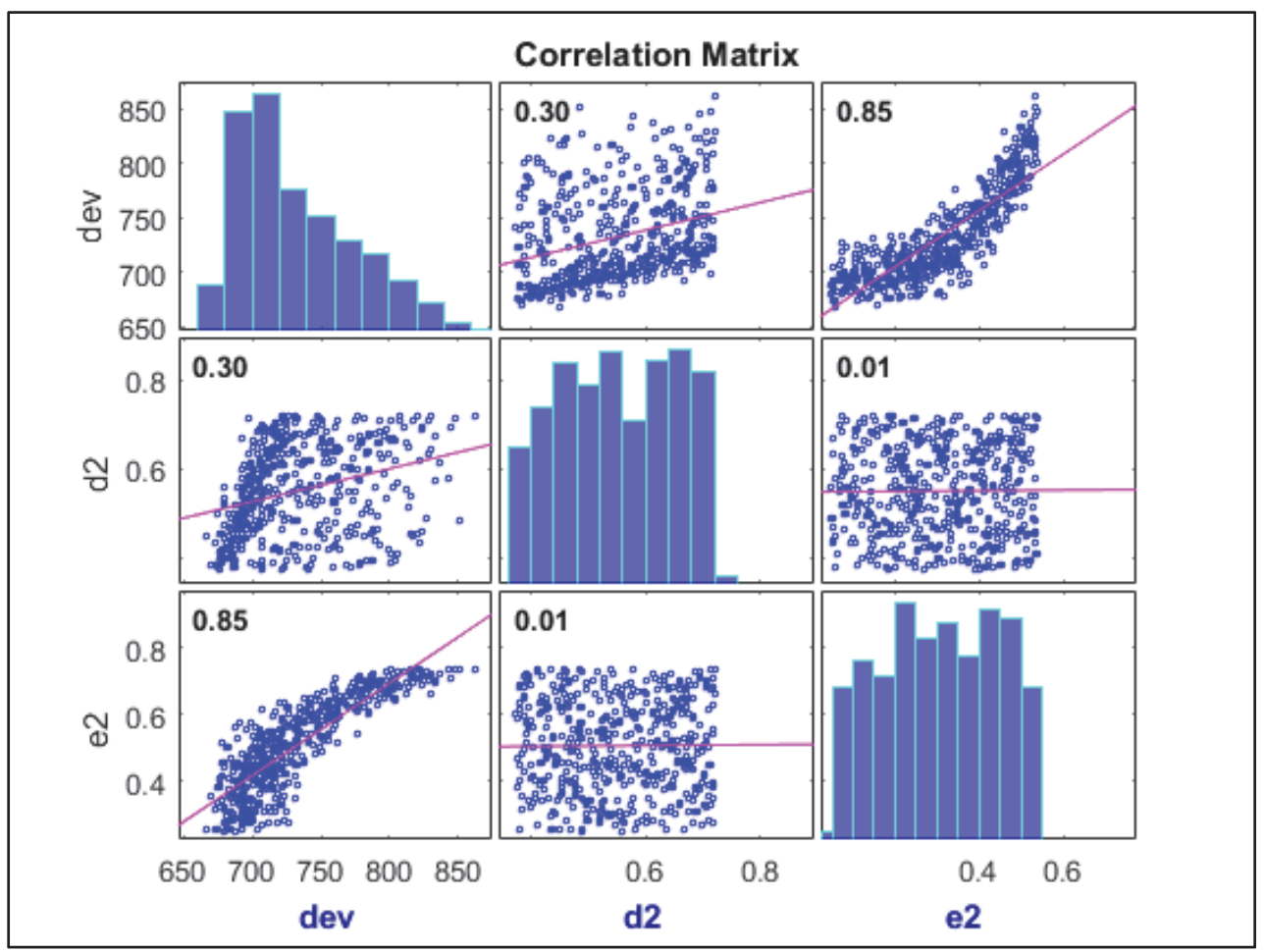

Figure B12 Histograms, Scatter Plots, and Correlation Coefficients for the Total Developed Land Area Percentage and its High-Sensitivity Parameters after 40-Year Simulation (Standardized Value) 


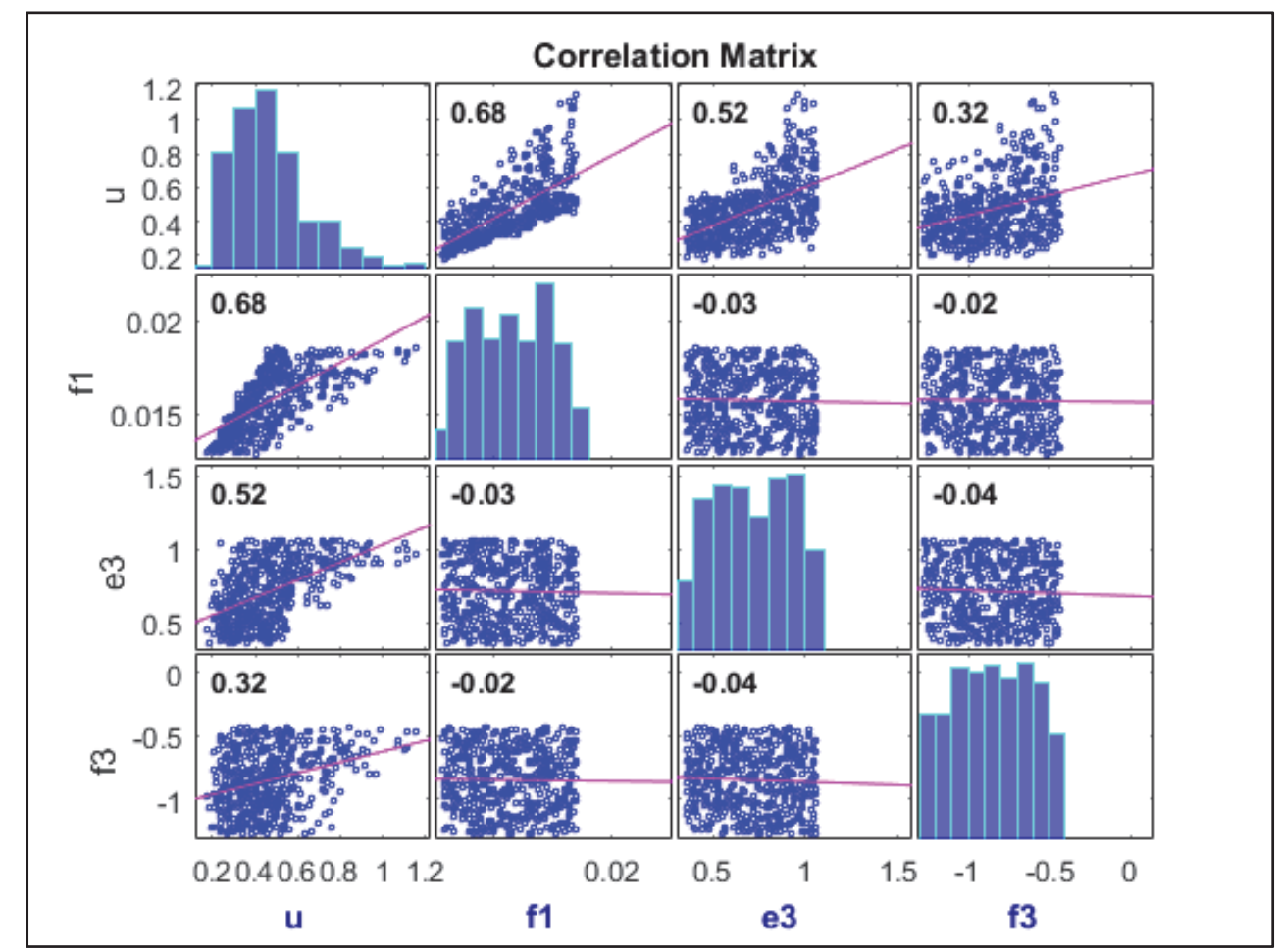

Figure B13 Histograms, Scatter Plots, and Correlation Coefficients between the Total Utility and its High-Sensitivity Parameters after 10-Year Simulation (Standardized Value)

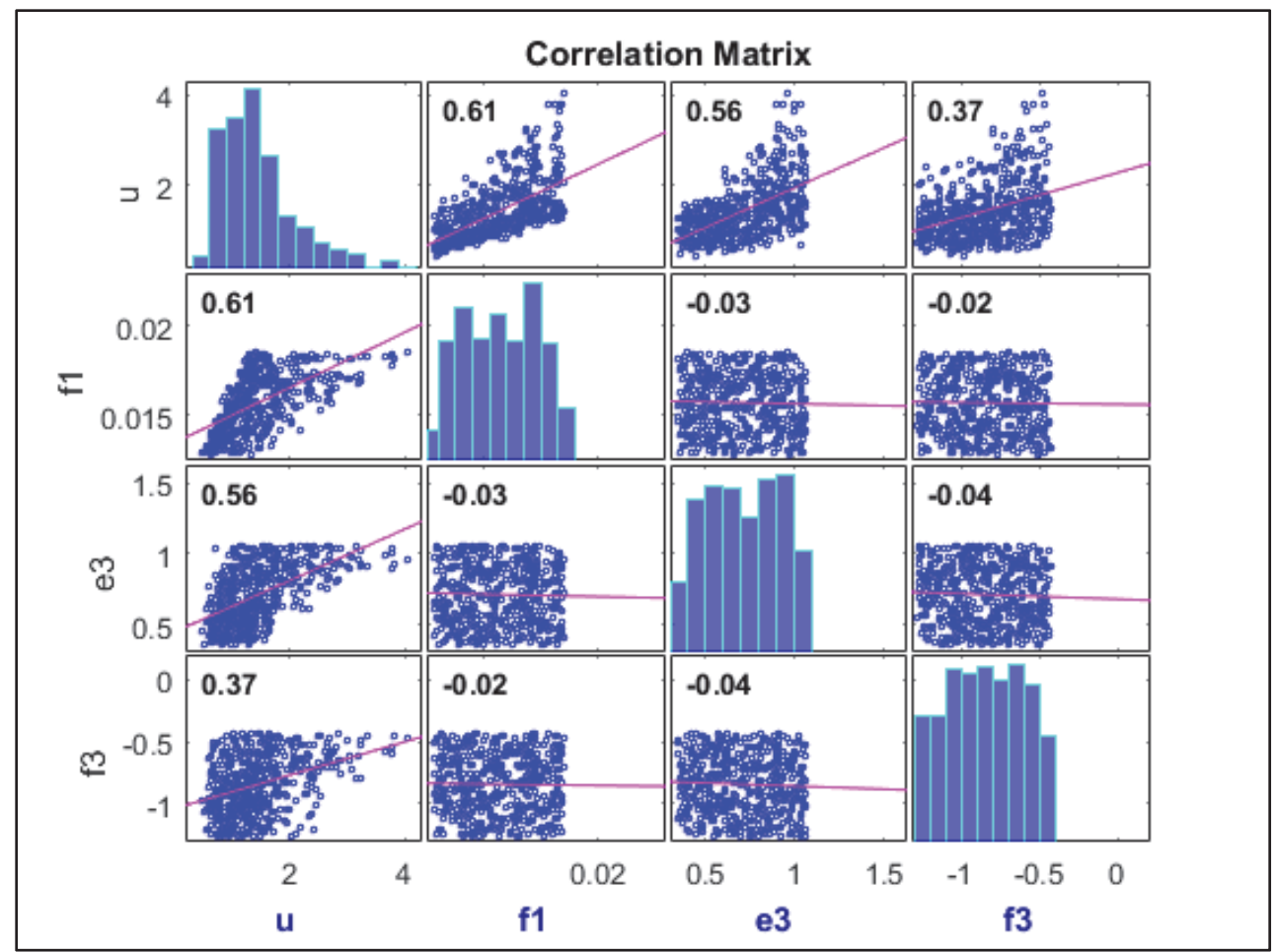

Figure B14 Histograms, Scatter Plots, and Correlation Coefficients between the Total Utility and its High-Sensitivity Parameters after 20-Year Simulation (Standardized Value) 


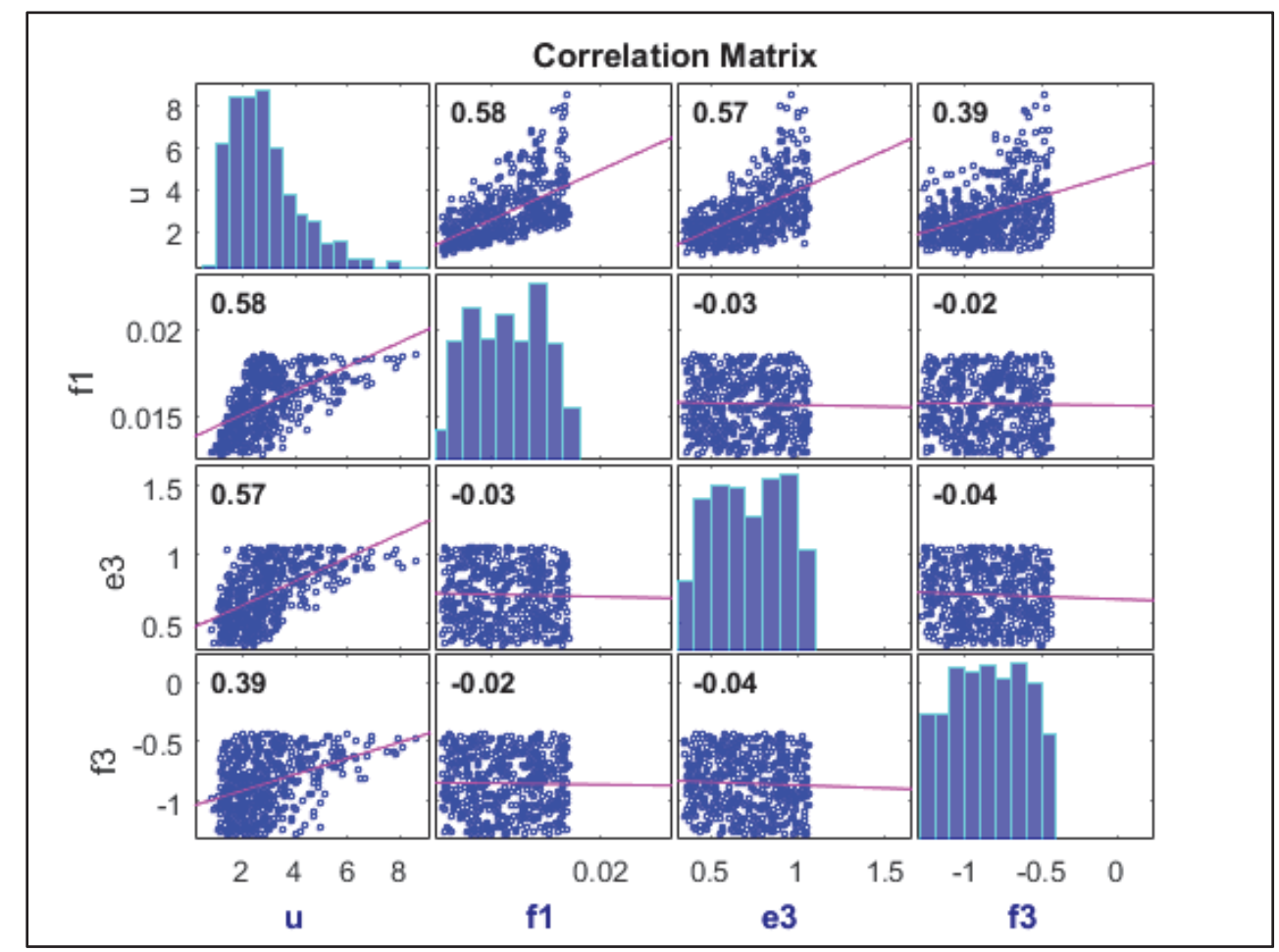

Figure B15 Histograms, Scatter Plots, and Correlation Coefficients between the Total Utility and its High-Sensitivity Parameters after 30-Year Simulation (Standardized Value)

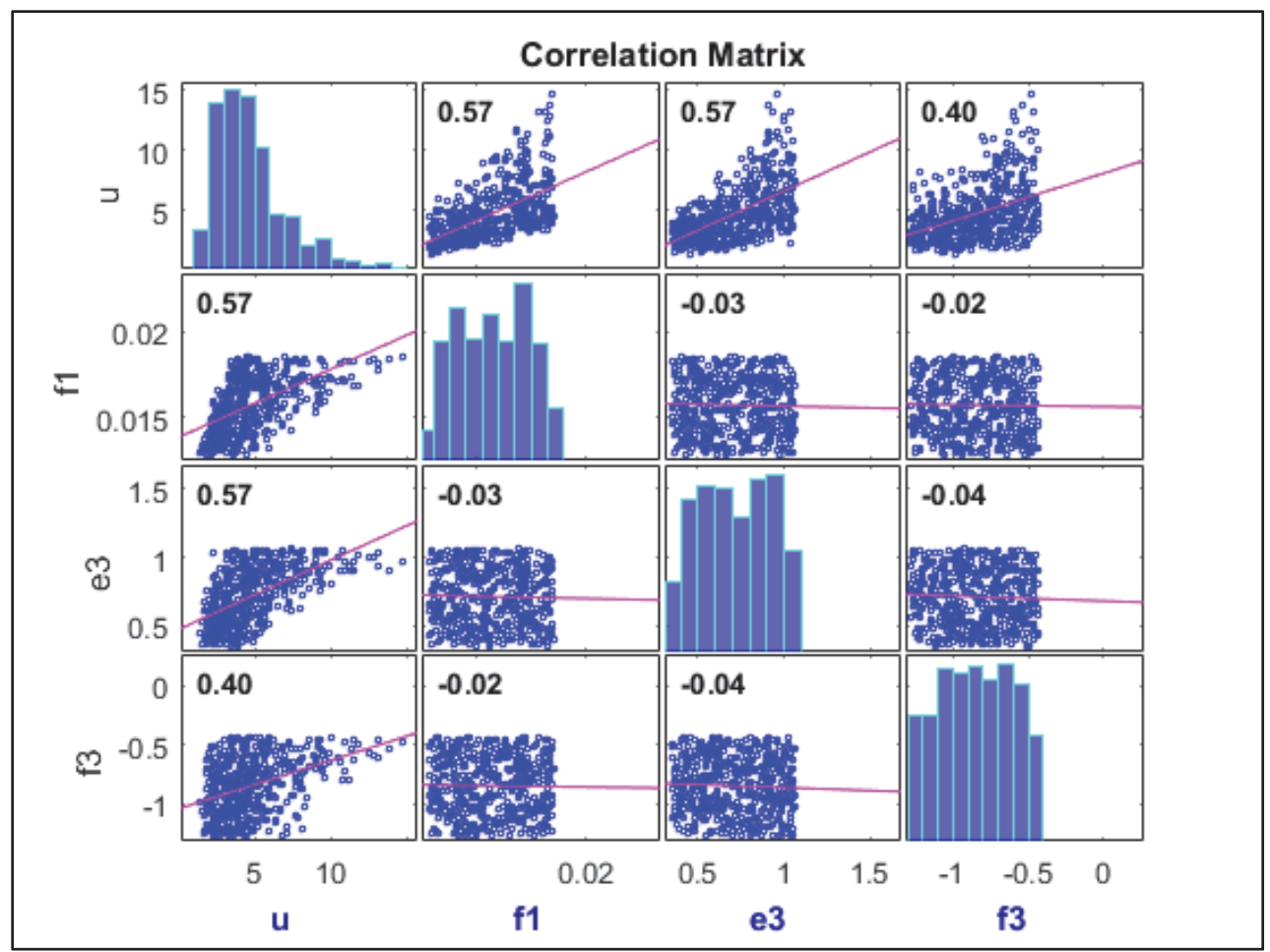

Figure B16 Histograms, Scatter Plots, and Correlation Coefficients between the Total Utility and its High-Sensitivity Parameters after 40-Year Simulation (Standardized Value) 


\section{VITA}

Kenan Li was born in Liaoyang, Liaoning in China. After completing his schoolwork at high school in Liaoyang in 2005, he entered Nankai University in Tianjin, China. He earned a Bachelor of Science degree in Environmental Sciences with a minor in Applied Mathematics in 2009, and attended the Louisiana State University as a graduate research assistant in the same year. He earned his Master of Science degree in Environmental Sciences in 2011 under the direction of Dr. Nina Lam, and enrolled in the Department of Oceanography and Coastal Sciences at Louisiana State University in the same year under the direction of Dr. Nina Lam and Dr. Kenneth Rose. He transferred from the Department of Oceanography and Coastal Sciences to the Department of Environmental Sciences in 2012 with Dr. Nina Lam as his graduate advisor. He will receive a Doctor of Philosophy degree in Environmental Sciences under the direction of Dr. Nina Lam, in December 2015. 